\title{
HALF-SPACE MACDONALD PROCESSES
}

\author{
GUILLAUME BARRAQUAND ${ }^{1}$, \\ ALEXEI BORODIN ${ }^{2,3}$ and IVAN CORWIN ${ }^{4}$ \\ ${ }^{1}$ Laboratoire de Physique de l'École Normale Supérieure, ENS, Université PSL, CNRS, \\ Sorbonne Université, Université de Paris, Paris, France; \\ email: barraquand@math.cnrs.fr \\ ${ }^{2}$ Department of Mathematics, MIT, Cambridge, USA \\ ${ }^{3}$ Institute for Information Transmission Problems, Moscow, Russia; \\ email: borodin@math.mit.edu \\ ${ }^{4}$ Columbia University, Department of Mathematics, \\ 2990 Broadway, New York, NY 10027, USA; \\ email: ivan.corwin@gmail.com
}

Received 9 March 2018; accepted 19 February 2020

\begin{abstract}
Macdonald processes are measures on sequences of integer partitions built using the Cauchy summation identity for Macdonald symmetric functions. These measures are a useful tool to uncover the integrability of many probabilistic systems, including the Kardar-Parisi-Zhang (KPZ) equation and a number of other models in its universality class. In this paper, we develop the structural theory behind half-space variants of these models and the corresponding half-space Macdonald processes. These processes are built using a Littlewood summation identity instead of the Cauchy identity, and their analysis is considerably harder than their full-space counterparts.

We compute moments and Laplace transforms of observables for general half-space Macdonald measures. Introducing new dynamics preserving this class of measures, we relate them to various stochastic processes, in particular the log-gamma polymer in a half-quadrant (they are also related to the stochastic six-vertex model in a half-quadrant and the half-space ASEP). For the polymer model, we provide explicit integral formulas for the Laplace transform of the partition function. Nonrigorous saddle-point asymptotics yield convergence of the directed polymer free energy to either the Tracy-Widom (associated to the Gaussian orthogonal or symplectic ensemble) or the Gaussian distribution depending on the average size of weights on the boundary.
\end{abstract}

2010 Mathematics Subject Classification: 60B99 (primary); 05E05, 82B23, 60B20, 82D60, 60K35, 35R60 (secondary) 


\section{Introduction}

In commencing the investigation that resulted in this paper, our goal was to prove limit theorems for the Kardar-Parisi-Zhang (KPZ) stochastic partial differential equation (PDE) [KPZ86, Cor12, Qua12] in a half-space [CS18], as well as for the log-gamma directed polymer [Sep12, COSZ14] in a halfquadrant [OSZ14]. Half-space systems are considerably more complicated to analyze than their full-space counterparts, and to us, the proper framework in which to initiate our study seemed to be that of half-space Macdonald processes (which we introduce here). Based on results from earlier analysis of zerotemperature models like TASEP and last-passage percolation in a half-space [BR01a, BR01b, BR01c, SI04, BBCS18b, BBNV18] (solvable via methods of Pfaffian point processes [BR05]), one may predict a rich phase diagram detailing the effect of the boundary strength on the fluctuation scalings and statistics. Despite previous efforts [TW13b, TW13a, OSZ14], there were no limit results known prior to our investigation (besides [BBCW18], which we developed with M. Wheeler in parallel to the present work). Even in the physics literature, the nonrigorous replica Bethe ansatz has proved difficult to apply, with results limited to two special boundary conditions for the KPZ equation (pure reflection [BBC16] or pure absorption [GLD12]).

In this paper, we develop the structural theory of half-space Macdonald processes and explore some of the rich hierarchy of limits and specializations. The theory of half-space Macdonald processes builds on the case of full-space Macdonald processes [BC14] but also employs a number of novel ideas.

Before highlighting the new ideas and challenges that arise in this half-space setting, we briefly recall the major developments in the full-space theory of Macdonald process (see also the reviews [BG12, Bor14, BP14, Cor14]).

Using operators to compute expectations. Applying operators that act diagonally on Macdonald polynomials to the normalizing constant for the measure yields a general mechanism to compute expectations of observables related to the operators' eigenvalues. This idea was introduced in [BC14], wherein Macdonald difference operators were used extensively, and it is developed further in [BCGS16, BG15, Dim18, GZ18].

$(2+1)$-dimensional Markovian dynamics. A general scheme to build Markovian dynamics on two-dimensional triangular arrays preserving the class of Schur processes (introduced in [Oko01, OR03]) was proposed in [BF14a, Bor11]. These push-block dynamics were studied in [BC14] in the Macdonald case, especially at the $q$-Whittaker level. 
Other dynamics preserving Macdonald processes connected to the RSK algorithm were studied at the Whittaker level in continuous time in [BP16, OP13] and in discrete time [MP17]. These RSK-type dynamics were also studied earlier at the Whittaker level in [O'C12, COSZ14] and later at the Hall-Littlewood level [BBW16, BP15, BM18].

Marginal Markov processes and their limits. Some marginals of these $(2+1)$-dimensional dynamics are themselves Markov processes. Some of these processes were new, while others have been introduced earlier. This relation has provided some new tools in their studies. Let us mention the $q$-TASEP [BC14, BCS14, BC13], $q$-Push(T)ASEP [CP15], log-gamma directed polymer [BCR13, COSZ14, Sep12], strict-weak polymer [CSS15, O015], O'Connell-Yor polymer [BC14, OY01, O'C12], KPZ equation [AKQ14, BG97, BCF14, BCFV15, KPZ86], Stochastic six-vertex model [BCG16, BBW16, GS92], HallLittlewood-PushTASEP [Gho17b] and ASEP [BCS14, B017, BM18].

Connections to random matrix theory. Relations between the coordinates of a random partition under the Macdonald measures (in particular, HallLittlewood) and random matrices were explored in [Bor95, Bor99, BG15, BP15, Ful02, GS15, GKV14].

Gibbsian line ensembles. After taking certain scaling limits, the algebra disappears but the integrability remains in the form of a Gibbs property; this is useful in extending one-point to process level asymptotics [BCT17, CD18, CH14, CH16, CT16].

Curious determinantal identities. In a few specific cases, curious determinantal identities allow us to relate certain functionals of the Macdonald measure with the Schur measure or other determinantal point processes [Agg18, Bor18, AB19, BBCW18, BO17, OP17]. This typically relates non-free-fermionic models to fermionic ones, and greatly simplifies the asymptotic analysis.

KPZ universality class asymptotics. For all the above mentioned models, the Laplace transform of observables of interest can be expressed as a Fredholm determinant whose asymptotic analysis leads to KPZ-type limit theorems [AB19, Bar15, BC14, BCF14, BCR13, BCFV15, B017, CSS15, FV15, Gho17b, KQ18, O015].

The story of (full-space) Macdonald processes is far from complete. Many challenges remain such as computing the asymptotic behavior for the entire 
measure, multipoint fluctuations (that is, convergence to Airy-type processes and line ensembles) and asymptotics away from the edge (corresponding to bulk eigenvalue statistics).

Given the success of Macdonald processes in studying systems like the KPZ equation, log-gamma polymer model and ASEP, it is only natural to seek an appropriate half-space version of the measures and associated theory. There is a natural starting point based on the Macdonald polynomial version of the Littlewood identities. However, there are difficulties-algebraic (we need new operators to compute expectations of certain observables of interest), probabilistic/combinatorial (we need new dynamics to deal with the boundary) and analytic (our formulas do not organize themselves into Fredholm determinants or Pfaffians). We overcome all of these, except for the analytic ones where we still manage to obtain the expected phase diagram for fluctuations via formal steepest descent analysis. Despite the lack of rigor in this last step, it is the first time that this full phase diagram has been accessed for these models (even in the physics literature).

We now provide a few details on each of these novelties. The reader not familiar with Macdonald processes may skip this part on the first reading and jump to Section 1.1.

Using Littlewood-type identities. The definition of half-space Macdonald process (Section 2.3) relies on a Macdonald analogue of the Littlewood symmetric function summation identity (24) from [Mac95] (The term 'process' versus 'measure' in 'Macdonald process' and 'Macdonald measure' distinguishes between measures on interlacing sequences of partitions and just on single partitions.). The $(q=t)$ half-space (or Pfaffian) Schur process was defined much earlier in [BR05, SI04] and studied at length in [BBCS18b] in connection to stochastic processes like half-space TASEP and last-passage percolation. The half-space Whittaker measure was introduced in [OSZ14] (the corresponding Littlewood identity is due to [Sta01]).

Markovian 'boundary' dynamics. In order to relate our half-space Macdonald process to interesting stochastic processes, we construct local Markovian dynamics on interlacing sequences of partitions, which preserve the class of Macdonald processes (that is, applying the dynamics to a sequence distributed according to one Macdonald process yields, at a later time, another sequence distributed according to a Macdonald process with modified parameters) and which have Markovian projections when restricted to a few first or last parts of the partitions (We will sometimes use 'Macdonald processes' as short 
for 'half-space Macdonald processes'. To avoid any ambiguity, we will always use precisely 'full-space Macdonald processes' when referring to the original Macdonald processes from [BC14].). The existence of such dynamics is far from evident. The novelty here (explained in Section 2.4) is finding appropriate dynamics at the boundary of the half-space.

Operators. In developing methods to compute distributional information about marginals of these measures, we construct a new operator (denoted by $\mathbf{M}_{n}^{z}$ in the text; see Section 3.5), which is an analytic continuation of a $q$-integral operator introduced by Noumi [NS12] (denoted by $\mathbf{N}_{n}^{z}$ or $\overline{\mathbf{N}}_{n}^{z}$ in the text; see Section 3.3) and which acts diagonally on Macdonald polynomials. By applying this operator to the normalizing function for the measure, we are able to prove a $(q, t)$-Laplace transform formula for $\lambda_{1}$ and $\lambda_{n}$ (where $\lambda_{1}$ and $\lambda_{n}$ are the first and last parts of the partition under the half-space Macdonald measure). The original Noumi operator cannot be used in computing the Laplace transform of $\lambda_{1}$ since in doing so we must interchange an infinite summation in the definition of the operator with an infinite summation in the normalizing function. This interchange is not justifiable and, in fact, leads to the wrong answer (see Remark 3.23). By working with the analytic continuation operator $\mathbf{M}_{n}^{z}$ (which is encoded in terms of Mellin-Barnes-type integrals with nice convergence properties), we may justifiably perform such an interchange. When the parameter $t=0$ (the case of $q$-Whittaker process), this yields a $q$-Laplace transform formula for $q^{-\lambda_{1}}$, which cannot be derived from moment formulas due to the ill-posedness of the moment problem for that random variable. We additionally prove many other moment formulas as well as a Laplace transform formulas using ideas that were present in [BC14, BCGS16].

Use of Plancherel specialization and a proof of [OSZ14] conjectural formulas. Taking the Macdonald parameters $t=0$ and $q \rightarrow 1$, and performing appropriate scaling on partitions leads to the half-space Whittaker process (this limit procedure is similar to that used in the full-space case in [BC14]). The dynamics we constructed at the top of the Macdonald hierarchy, when restricted to $\lambda_{1}$, converge to the recursion for partition functions satisfied by the half-space log-gamma polymer model. This model was studied previously in [OSZ14] by way of applying the geometric RSK correspondence to a symmetric weight matrix. That approach only related the polymer partition function on the diagonal (that is, at the boundary of the domain) to the Whittaker measure. Our 
dynamic approach readily relates the partition function in the entire half-space to an appropriate Whittaker measure. This ability to work off the diagonal is, in fact, key-it allows us to introduce a 'Plancherel' specialization into our Whittaker measure, which drastically improves the decay properties of the Whittaker measure density and ultimately allows us to rigorously derive Laplace transform formulas for the associated polymer model. The polymer model that comes from this Plancherel component is a mix of the log-gamma and O'Connell-Yor polymers (as also considered in [BCFV15] in the full-space setting). Having established formulas for the mixed model, we can then shrink the Plancherel component to zero and by continuity we arrive at formulas for the log-gamma polymer alone. Without the inclusion of a Plancherel component, the relevant Whittaker measure formulas do not have sufficient decay to rigorously justify the derivation of the Laplace transform. In fact, in [OSZ14], the authors performed formal (that is, neglecting issues of convergence and the applicability of the Whittaker Plancherel theory) calculations to derive a Laplace transform formula (both formulas (5.15) and (5.16) in [OSZ14]). They remarked, 'It seems reasonable to expect the integral formulas (5.15) and (5.16) to be valid, at least in some suitably regularized sense.' We prove these formulas as Corollary 6.41.

Two types of Laplace transform formulas. We prove two types of halfspace log-gamma polymer Laplace transform formulas. The first type (Corollaries 6.40 and 6.41, coming from Theorem 6.38) is in terms of a single $n$-fold contour integral (and is in the spirit of the speculative formulas from [OSZ14] as well as formulas proved in the full-space case in [COSZ14]). The second type, Theorems 3.12 and 3.20, is in terms of a (finite) series of increasing dimensional integrals. Though we are unable to write this as a Fredholm Pfaffian, it is the half-space version of the Fredholm determinant expansion formulas that arise in [BCR13] and proved, therein, quite useful for asymptotics. We attempted to perform asymptotics (taking the system size $n \rightarrow \infty$ ) using these formulas. At the level of studying the term-by-term limit around the critical points of the integrals, we demonstrate convergence to the expected Fredholm Pfaffian expansions that govern the halfspace KPZ universality class one-point fluctuation phase diagram. Unfortunately, the presence of certain gamma function ratios preclude establishing sufficient control over the tails of the integrals as well as the series, as would be necessary to rigorously prove our convergence results. Recently, asymptotics of flat initial data ASEP [OQR17] and 
multipoint formulas for polymers [NZ16] have likewise been stymied by similar considerations, which have prevented rigorous asymptotics, despite formal critical point results agreeing with predictions.

Pfaffian identities. Thus, we fall short of our initial goal of proving asymptotic limit theorems. There is, however, one exception. In joint work [BBCW18] with Wheeler-which came as an outgrowth of the present project and [BBW16, WZJ16]—we found that for a special case of the half-space Hall-Littlewood process (itself, a special case of the Macdonald process when $q=0$, which relates to the half-quadrant stochastic six-vertex model) the Laplace transform has an alternative expression in terms of a related Pfaffian point process (the half-space or Pfaffian Schur process [BR05]). Through this identity and known asymptotic techniques for Pfaffian point processes, we were able to rigorously prove the desired type of KPZ universality class asymptotics. The presence of Pfaffians in this Laplace transform representation encapsulates some crucial cancellations, which are not apparent in our series expression. Unfortunately, this relationship to a Pfaffian point process is presently mysterious and it is unclear if it generalizes beyond the one special case (see Section 5.3 for more details).

\subsection{Half-space Macdonald measures and processes. Half-space} Macdonald measures, defined more precisely in Section 2.3, are probability distributions on integer partitions $\lambda=\left(\lambda_{1} \geqslant \lambda_{2} \geqslant \cdots \geqslant 0\right)$ for which (the notation will be explained below)

$$
\mathbb{P}^{q, t}(\lambda)=\frac{1}{Z\left(\rho, \rho^{\swarrow}\right)} P_{\lambda}(\rho) \mathcal{E}_{\lambda}\left(\rho^{\swarrow}\right) .
$$

Here $P_{\lambda}$ are the Macdonald symmetric functions [Mac95, Ch. VI] depending on two parameters $q, t$ (we assume that these parameters take values in $[0,1$ ) throughout) and $\mathcal{E}_{\lambda}$ is another symmetric function defined by

$$
\mathcal{E}_{\lambda}=\sum_{\mu^{\prime} \text { even }} b_{\mu}^{\mathrm{el}} Q_{\lambda / \mu}
$$

where $P_{\lambda / \mu}, Q_{\lambda / \mu}$ are skew Macdonald symmetric functions, $b_{\mu}^{\mathrm{el}}$ are explicit $(q, t)$-dependent coefficients and the sum is over all partitions, which are dual even (meaning that $\mu_{2 i-1}=\mu_{2 i}$ for all $i$ ). The symbols $\rho^{\swarrow}$ and $\rho$ represent specializations of the algebra of symmetric functions that can depend on many parameters, and $Z\left(\rho, \rho^{\swarrow}\right)$ is the normalizing constant that is necessary to make 
$\mathbb{P}^{q, t}$ a probability measure. Section 2.2 provides more details for all of these objects.

Half-space Macdonald processes (Definition 2.3) are probability measures on sequences of partitions whose marginals are half-space Macdonald measures. Macdonald symmetric functions usually take a different name when their parameters $q, t$ are specialized to certain values. We will name our half-space processes accordingly. The chart in Figure 1 depicts the hierarchy of these degenerations and the relations between most integrable half-space systems discussed in this paper. We will use $\mathbb{P}^{q, t}, \mathbb{E}^{q, t}$ to denote the probability measure and expectation operator for the Macdonald process, and $\mathbb{P}^{q, t}, \mathbb{E}^{q, t}$ for the Macdonald measure. Setting $t=0$ and $q$ general results in the $q$-Whittaker case and we write $\mathbb{P}^{q}, \mathbb{E}^{q}$, where as setting $q=0$ and $t$ general results in the Hall-Littlewood case and we likewise write $\mathbb{P}^{t}, \mathbb{E}^{t}$. When we consider further degenerations (for example, the Whittaker case, ASEP etc), we will denote the probability measure and expectation by $\mathbb{P}, \mathbb{E}$.

1.2. Computing expectations of observables. At the Macdonald level, we are able to compute integral formulas for various moments and Laplace-type transforms. A general scheme for computing expectations of certain observables of Macdonald measures was introduced in [BC14, Section 2.2.3], and we develop this into the half-space setting. As alluded to earlier, in order to compute one of our $(q, t)$-Laplace transform formulas-see Theorem B-we need to introduce a new operator $\mathbf{M}_{n}^{z}$, which extends the action of $\overline{\mathbf{N}}^{z}$ from polynomials but has better analytic properties. We briefly review our main Laplace transform results (we leave the moment formulas, which are in the spirit of the earlier fullspace work of [BC14] to the main text-see Section 3).

Consider real variables $x_{1}, \ldots, x_{n} \in(0,1)$ and a specialization $\rho$. We have the (Littlewood) identity

$$
\sum_{\lambda} P_{\lambda}\left(x_{1}, \ldots, x_{n}\right) \mathcal{E}_{\lambda}(\rho)=Z\left(x_{1}, \ldots x_{n} ; \rho\right)
$$

where the function $Z\left(x_{1}, \ldots x_{n} ; \rho\right)$ has an explicit form (see Section 2.2). We recognize on the left-hand side the unnormalized density of the half-space Macdonald measure.

Assume that we have a linear operator $\mathbf{A}_{n}$ acting on functions in variables $x_{1}, \ldots, x_{n}$, which is diagonal in the basis of symmetric polynomials $\left\{P_{\lambda}\right\}$ with eigenvalues $\left\{d_{\lambda}\right\}$. Applying $\mathbf{A}_{n}$ to both sides of the above identity and subsequently dividing both sides by the normalizing constant $Z$, we find that 


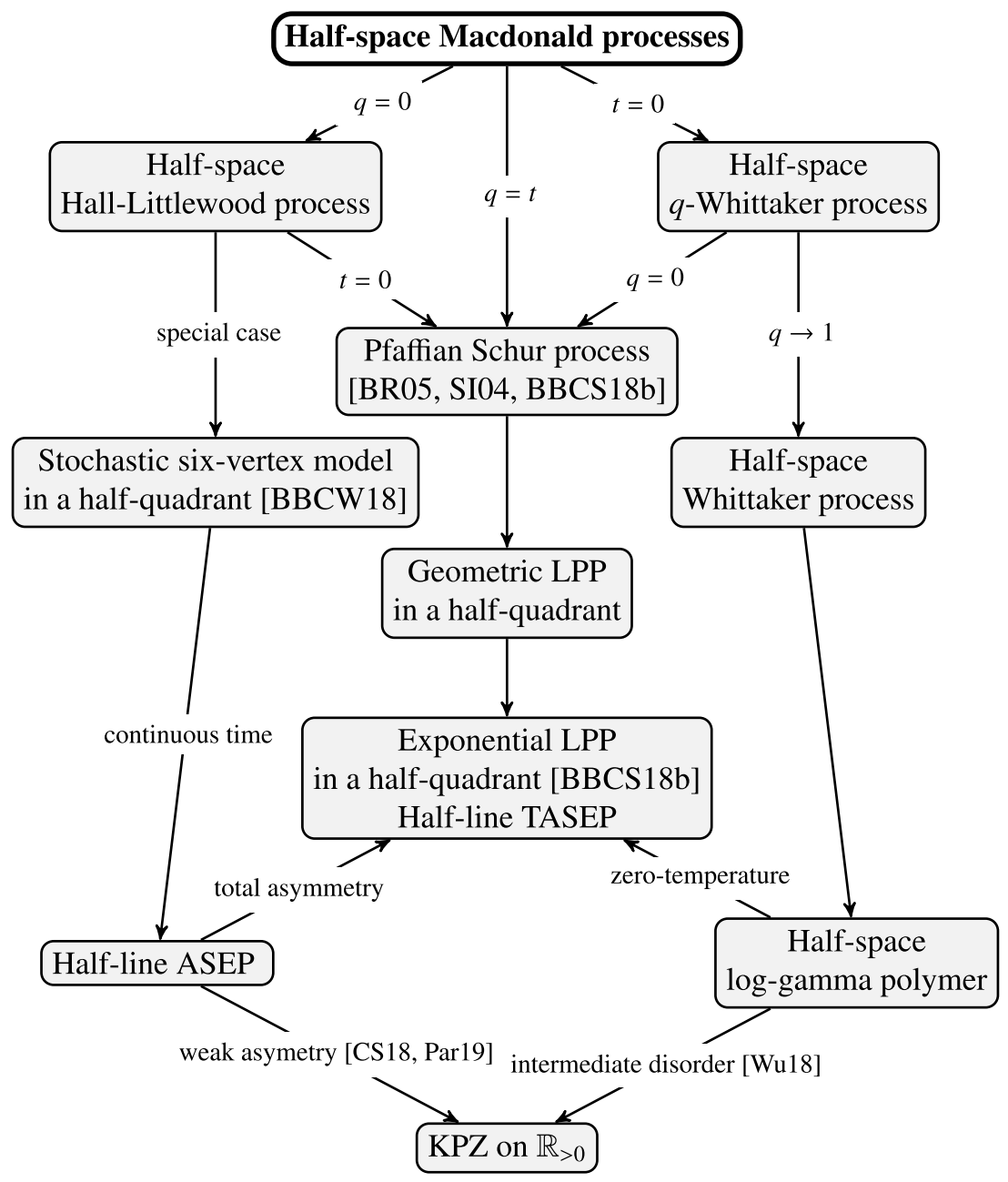

Figure 1. Hierarchy of half-space Macdonald processes and their degenerations. The arrows mean that one has to take a specialization of parameters or a scaling limit.

for the half-space Macdonald measure with $\rho=\left(x_{1}, \ldots, x_{n}\right)$ and $\rho^{\swarrow}=\rho$,

$$
\mathbb{E}^{q, t}\left[d_{\lambda}\right]=\sum_{\lambda \in \mathbb{Y}} d_{\lambda} \frac{P_{\lambda}\left(x_{1}, \ldots, x_{n}\right) \mathcal{E}_{\lambda}(\rho)}{Z\left(x_{1}, \ldots x_{n} ; \rho\right)}=\frac{\mathbf{A}_{n} Z\left(x_{1}, \ldots x_{n} ; \rho\right)}{Z\left(x_{1}, \ldots x_{n} ; \rho\right)},
$$


though one needs to justify that the infinite summation commutes with $\mathbf{A}_{n}$. We may also iterate the procedure to compute more complicated observables.

Such operators diagonalized by Macdonald polynomials are available. In particular, we will use Macdonald difference operators $\mathbf{D}_{n}^{r}$, and a variant of them that we denote $\overline{\mathbf{D}}_{n}^{r}$, for which

$$
\mathbf{D}_{n}^{r} P_{\lambda}=e_{r}\left(q^{\lambda_{1}} t^{n-1}, \ldots, q^{\lambda_{n}} t^{0}\right) P_{\lambda}, \quad \overline{\mathbf{D}}_{n}^{r} P_{\lambda}=e_{r}\left(q^{-\lambda_{1}} t^{1-n}, \ldots, q^{-\lambda_{n}} t^{0}\right) P_{\lambda},
$$

where $e_{r}$ is the $r$ th elementary symmetric function. We will also use Noumi's $q$-integral operator $\mathbf{N}_{n}^{z}$ (see Section 3.3), and a variant of it denoted by $\overline{\mathbf{N}}_{n}^{z}$, for which

$$
\mathbf{N}_{n}^{z} P_{\lambda}=\prod_{i=1}^{n} \frac{\left(q^{\lambda_{i}} t^{n-i+1} z ; q\right)_{\infty}}{\left(q^{\lambda_{i}} t^{n-i} z ; q\right)_{\infty}} P_{\lambda}, \quad \overline{\mathbf{N}}_{n}^{z} P_{\lambda}=\prod_{i=1}^{n} \frac{\left(q^{-\lambda_{i}} t^{i} z ; q\right)_{\infty}}{\left(q^{-\lambda_{i}} t^{i-1} z ; q\right)_{\infty}} P_{\lambda},
$$

where $(x)_{\infty}=(x ; q)_{\infty}=\prod_{i \geqslant 0}\left(1-q^{i} x\right)$. Following arguments similar to [BC14, BCGS16], we can make the above approach entirely rigorous for operators $\mathbf{D}_{n}^{r}$, $\overline{\mathbf{D}}_{n}^{r}$ and $\mathbf{N}_{n}^{z}$, and the action of these operators on the normalizing constant can be expressed in terms of contour integrals (see Sections 3.2 and 3.4). Regarding the $q$-integral operator $\overline{\mathbf{N}}_{n}^{z}$, equation (1) is not true since we cannot justify moving the operator inside the sum that defines $Z$ (in fact, this interchange is not true due to a lack of convergence when one tries to use Fubini). This is unfortunate because the quantity

$$
\mathbb{E}^{q, t}\left[\prod_{i=1}^{n} \frac{\left(q^{-\lambda_{i}} t^{i} z\right)_{\infty}}{\left(q^{-\lambda_{i}} t^{i-1} z\right)_{\infty}}\right]
$$

is exactly what we need to compute in order to study the partition function of the log-gamma polymer. This is why we introduce a different integral operator $\mathbf{M}_{n}^{z}$, which coincides with $\overline{\mathbf{N}}_{n}^{z}$ on polynomials, but not on $Z\left(x_{1}, \ldots, x_{n} ; \rho\right)$, and has better analytic properties. This is the main technical novelty of the present paper regarding the computation of observables.

Let $a_{1}, \ldots, a_{n}$ be parameters in $(0,1)$. Consider the half-space Macdonald measure with specializations $\rho=\left(a_{1}, \ldots, a_{n}\right)$ and $\rho^{\swarrow}=\rho$. We further assume that the parameters $a_{1}, \ldots, a_{n}$ are close enough to each other (see the statements of Theorem 3.12 and Theorem 3.20 for precise statements).

TheOREM A (Theorem 3.12). Let $z \in \mathbb{C} \backslash \mathbb{R}_{>0}$. We have

$$
\mathbb{E}^{q, t}\left[\prod_{i=1}^{n} \frac{\left(q^{\lambda_{i}} t^{n-i+1} z\right)_{\infty}}{\left(q^{\lambda_{i}} t^{n-i} z\right)_{\infty}}\right]
$$




$$
\begin{aligned}
= & \sum_{k=0}^{n} \frac{1}{k !} \int_{R-\mathbf{i} \infty}^{R+\mathbf{i} \infty} \frac{d s_{1}}{2 \mathbf{i} \pi} \cdots \int_{R-\mathbf{i} \infty}^{R+\mathbf{i} \infty} \frac{d s_{k}}{2 \mathbf{i} \pi} \oint \frac{d w_{1}}{2 \mathbf{i} \pi} \cdots \oint \frac{d w_{k}}{2 \mathbf{i} \pi} \\
& \mathcal{A}_{\vec{s}}^{q, t}(\vec{w}) \prod_{i=1}^{k} \Gamma\left(-s_{i}\right) \Gamma\left(1+s_{i}\right) \\
& \times \prod_{i=1}^{k} \frac{\mathcal{G}^{q, t}\left(w_{i}\right)}{\mathcal{G}^{q, t}\left(q^{s_{i}} w_{i}\right)} \frac{\phi\left(w_{i}^{2}\right)(-z)^{s_{i}}}{\phi\left(q^{s_{i}} w_{i}^{2}\right)\left(q^{s_{i}}-1\right) w_{i}},
\end{aligned}
$$

where $R \in(0,1)$ is chosen such that $0<q^{R}<a_{i} / a_{j}$ for all $i, j$, the positively oriented integration contours for the variables $w_{j}$ enclose all the $a_{i}$ 's and no other singularity, and we have used the shorthand notations

$$
\mathcal{A}_{\vec{s}}^{q, t}(\vec{w}):=\prod_{1 \leqslant i<j \leqslant k} \frac{\left(q^{s_{j}} w_{j}-q^{s_{i}} w_{i}\right)\left(w_{i}-w_{j}\right) \phi\left(q^{s_{i}+s_{j}} w_{i} w_{j}\right) \phi\left(w_{i} w_{j}\right)}{\left(q^{s_{i}} w_{i}-w_{j}\right)\left(q^{s_{j}} w_{j}-w_{i}\right) \phi\left(q^{s_{i}} w_{i} w_{j}\right) \phi\left(q^{s_{j}} w_{j} w_{i}\right)}
$$

and

$$
\mathcal{G}^{q, t}(w)=\prod_{j=1}^{n} \frac{\phi\left(w / a_{j}\right)}{\phi\left(w a_{j}\right)} \frac{1}{\Pi(w ; \rho)}, \quad \phi(z)=\frac{(t z)_{\infty}}{(z)_{\infty}},
$$

with $\Pi(w ; \rho)=\sum_{\lambda} P_{\lambda}(w) Q_{\lambda}(\rho)$ (see (29) for an explicit expression).

THEOREM B (Theorem 3.20). Let $z \in \mathbb{C} \backslash \mathbb{R}_{>0}$. Under mild assumptions on the specialization $\rho$, we have

$$
\begin{aligned}
& \mathbb{E}_{\left(a_{1}, \ldots, a_{n}\right), \rho}^{q, t}\left[\prod_{i=1}^{n} \frac{\left(q^{-\lambda_{i}} t^{i} z\right)_{\infty}}{\left(q^{-\lambda_{i}} t^{i-1} z\right)_{\infty}}\right]= \\
& \sum_{k=0}^{n} \frac{1}{k !} \int_{R-\mathbf{i} \infty}^{R+\mathbf{i} \infty} \frac{d s_{1}}{2 \mathbf{i} \pi} \cdots \int_{R-\mathbf{i} \infty}^{R+\mathbf{i} \infty} \frac{d s_{k}}{2 \mathbf{i} \pi} \\
& \oint \frac{d w_{1}}{2 \mathbf{i} \pi} \cdots \oint \frac{d w_{k}}{2 \mathbf{i} \pi} \mathcal{A}_{-\vec{s}}^{q, t}(\vec{w}) \prod_{i=1}^{k} \Gamma\left(-s_{i}\right) \Gamma\left(1+s_{i}\right) \\
& \times \frac{\overline{\mathcal{G}}^{q, t}\left(w_{i}\right)}{\overline{\mathcal{G}}^{q, t}\left(q^{-s_{i}} w_{i}\right)} \frac{\phi\left(w_{i}^{2}\right)(-z)^{s_{i}}}{\phi\left(q^{-s_{i}} w_{i}^{2}\right)\left(1-q^{s_{i}}\right) w_{i}},
\end{aligned}
$$

where $R \in(0,1)$ is chosen such that $a_{i}<q^{R}<a_{i} / a_{j}$ for all $i, j$, the positively oriented contours for the variables $w_{j}$ enclose all the $a_{i}$ 's and no other singularity, and we have used the shorthand notation

$$
\overline{\mathcal{G}}^{q, t}(w)=\prod_{j=1}^{n} \frac{\phi\left(a_{j} / w\right)}{\phi\left(w a_{j}\right)} \frac{1}{\Pi(w ; \rho)}
$$


The observables appearing in (2) and (3) above should be thought of as Laplace transforms. When $t=0$ and $q \rightarrow 1$, these will become exactly Laplace transforms of the random variables that we want to study. To be more precise, take $t=0$ for simplicity; using the $q$-binomial theorem (12), the left-hand side of (2) becomes

$$
\mathbb{E}^{q}\left[\frac{1}{\left(q^{\lambda_{n}} z\right)_{\infty}}\right]=\sum_{k=0}^{\infty} \frac{z^{k} \mathbb{E}^{q}\left[q^{k \lambda_{n}}\right]}{k !_{q}},
$$

where $k !{ }_{q}$ denotes the $q$-deformed factorial (see (11)).

The proof of Theorem B is significantly more delicate than the proof of Theorem A. This is because the left-hand side of (3) does not expand as a power series in $z$. Indeed (we take again the case $t=0$ for simplicity of the exposition),

$$
\mathbb{E}^{q}\left[\frac{1}{\left(q^{-\lambda_{1}} z\right)_{\infty}}\right] \neq \sum_{k=0}^{\infty} \frac{z^{k} \mathbb{E}^{q}\left[q^{-k \lambda_{1}}\right]}{k !_{q}} .
$$

Actually, the moments of $q^{-\lambda_{1}}$ grow too fast to determine the distribution uniquely (and sometimes they do not even exist). Formally taking a moment generating series of moments would not yield the correct result (see Remarks 3.23 and 4.11). This is why we need to work with the integral operator $\mathbf{M}^{z}$ instead of the $q$-integral operator $\overline{\mathbf{N}}^{z}$ in the proof of Theorem B in Section 3.4.

A similar moment problem issue came up in the study of full-space Macdonald processes, and [BCFV15] developed an involved argument (using formal power series in the variables of the $Q$ Macdonald polynomial) to prove the $q$-Laplace transform formula. That argument, however, cannot be applied here as there is no $Q$ polynomial or extra set of variables in which to expand. Thus, our new operator $\mathbf{M}_{n}^{z}$ provides the only apparent route to prove Theorem B. It also provides an alternative to the approach of [BCFV15] in the full-space case.

\subsection{Models related to half-space Macdonald processes.}

1.3.1. Log-gamma polymer in a half-quadrant. The log-gamma directed polymer model was introduced in [Sep12] and further studied in [BCR13, COSZ14, GRASY15, GS13, GRAS16, Gra17, OSZ14, NZ16, TLD14]. We consider a variant residing in a half-quadrant of $\mathbb{Z}^{2}$.

DEFINITION 1.1 (Half-space log-gamma polymer). Let $\alpha_{1}, \alpha_{2}, \ldots$ be positive parameters and $\alpha_{\circ} \in \mathbb{R}$ be such that $\alpha_{i}+\alpha_{\circ}>0$ for all $i \geqslant 1$. The half-space log-gamma polymer is a probability measure on up-right paths confined in the 


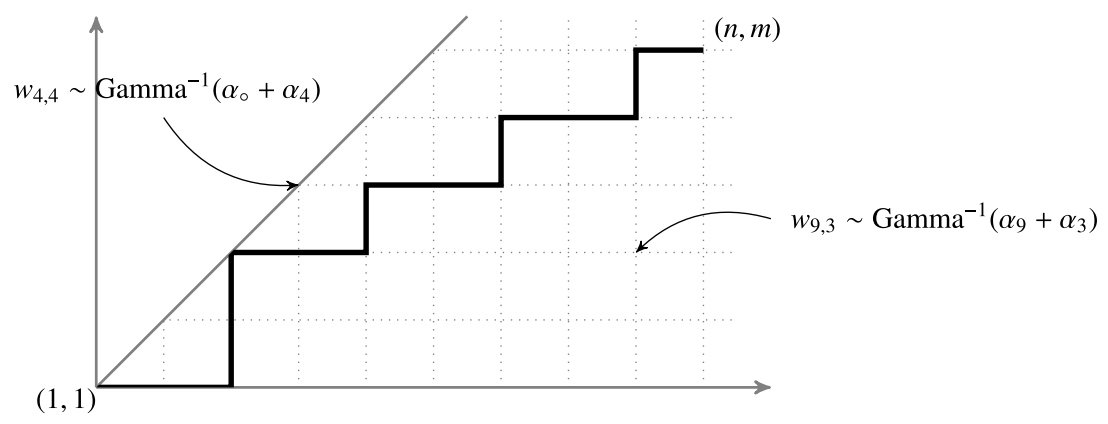

Figure 2. An admissible path in the half-space log-gamma polymer.

half-quadrant $\left\{(i, j) \in \mathbb{Z}_{>0}^{2}: i \geqslant j\right\}$ (see Figure 2), where the probability of an admissible path $\pi$ between $(1,1)$ and $(n, m)$ is given by

$$
\frac{1}{Z_{n, m}} \prod_{(i, j) \in \pi} w_{i, j},
$$

and where $\left(w_{i, j}\right)_{i \geqslant j}$ is a family of independent random variables such that for $i>j, w_{i, j} \sim \mathrm{Gamma}^{-1}\left(\alpha_{i}+\alpha_{j}\right)$ and $w_{i, i} \sim \mathrm{Gamma}^{-1}\left(\alpha_{\circ}+\alpha_{i}\right)$. The notation $\mathrm{Gamma}^{-1}(\theta)$ denotes the inverse of a Gamma distributed random variable with shape parameter $\theta$ (see Definition 6.24). The partition function $Z(n, m)$ is given by

$$
Z(n, m)=\sum_{\pi:(1,1) \rightarrow(n, m)} \prod_{(i, j) \in \pi} w_{i, j} .
$$

We show (see Proposition 6.34 below) that the observable $q^{-\lambda_{1}}$ admits a limit to $Z(t, n)$ when the parameters of the half-space Macdonald measure are scaled correctly, $t=0$ and $q$ goes to 1 in an appropriate manner. This limit corresponds to the half-space Whittaker process discussed in Section 6. To prove this result, we study Markovian dynamics preserving half-space Macdonald processes (actually, $q$-Whittaker processes) and interpret them in terms of new integrable particle systems (see Section 4.3). We then show (following arguments from [MP17]) that under these dynamics, $q^{-\lambda_{1}}$ satisfies a recurrence relation, which, in the $q \rightarrow 1$ limit, relates it to the half-space log-gamma polymer partition function.

Taking degenerations of integral formulas obtained for general Macdonald measures, we obtain the following moment formula (see Corollary 6.36). For 
$t \geqslant n$ and $k \in \mathbb{Z}_{>0}$ such that $k<\min \left\{2 \alpha_{i}, \alpha_{i}+\alpha_{\circ}\right\}$,

$$
\begin{aligned}
\mathbb{E}\left[Z(t, n)^{k}\right]= & \oint \frac{d w_{1}}{2 \mathbf{i} \pi} \cdots \oint \frac{d w_{k}}{2 \mathbf{i} \pi} \prod_{1 \leqslant a<b \leqslant k} \frac{w_{a}-w_{b}}{w_{a}-w_{b}-1} \frac{1+w_{a}+w_{b}}{2+w_{a}+w_{b}} \\
& \times \prod_{m=1}^{k} \frac{1+2 w_{m}}{1+w_{m}-\alpha_{\circ}} \prod_{i=1}^{t}\left(\frac{1}{\alpha_{i}-w_{m}-1}\right) \prod_{j=1}^{n}\left(\frac{1}{w_{m}+\alpha_{j}}\right),
\end{aligned}
$$

where the contours are such that for all $1 \leqslant c \leqslant k$, the contour for $w_{c}$ encloses $\left\{-\alpha_{j}\right\}_{1 \leqslant j \leqslant n}$ and $\left\{w_{c+1}+1, \ldots, w_{k}+1\right\}$ and excludes the poles of the integrand at $\alpha_{\circ}-1$ and $\alpha_{j}-1$ (for $1 \leqslant j \leqslant t$ ). Note that if $k>\alpha_{i}+\alpha_{j}$ or $k>\alpha_{i}+\alpha_{\circ}$ for some $i<j$, the $k$ th moment of $Z(t, n)$ fails to exist.

In order to go around certain technical issues, we also define a hybrid polymer model corresponding to a sort of convolution of the half-space log-gamma polymer and the O'Connell-Yor semidiscrete Brownian polymer. We will not give its exact definition for the moment but refer the reader to Definition 6.33 for the details. We denote its partition function by $Z(t, n, \tau)$, where $\tau$ is a positive parameter (which correspond to the time in the O'Connell-Yor polymer). The random variable $Z(t, n, \tau)$ weakly converges to the log-gamma partition function $Z(t, n)$ as $\tau$ goes to zero so that $Z(t, n, \tau)$ can be thought of as a regularization of $Z(t, n)$.

The moments of the partition function $Z(t, n)$ (and $Z(t, n, \tau)$ as well) grow too fast to determine its distribution uniquely. Nonetheless, by taking appropriate degenerations of Theorem $\mathrm{B}$, we are able to characterize the distribution of $Z(t$, $n$ ) via the following Laplace transform formula, which is proved as Corollary 6.20 in the text (or more precisely, a consequence of it stated as (171)).

If the parameters $\alpha_{i}>0$ are sufficiently close to each other, for any $t \geqslant n \geqslant 1$, $\tau>0$ and $u>0$,

$$
\begin{aligned}
\mathbb{E}\left[e^{-u Z(t, n, \tau)}\right]= & \sum_{k=0}^{n} \frac{1}{k !} \int_{R-\mathbf{i} \infty}^{R+\mathbf{i} \infty} \frac{d z_{1}}{2 \mathbf{i} \pi} \cdots \int_{R-\mathbf{i} \infty}^{R+\mathbf{i} \infty} \frac{d z_{k}}{2 \mathbf{i} \pi} \oint \frac{d v_{1}}{2 \mathbf{i} \pi} \cdots \oint \frac{d v_{k}}{2 \mathbf{i} \pi} \\
& \times \prod_{1 \leqslant i<j \leqslant k} \frac{\left(z_{i}-z_{j}\right)\left(v_{i}-v_{j}\right) \Gamma\left(v_{i}+v_{j}\right) \Gamma\left(-z_{i}-z_{j}\right)}{\left(z_{j}+v_{i}\right)\left(z_{i}+v_{j}\right) \Gamma\left(v_{j}-z_{i}\right) \Gamma\left(v_{i}-z_{j}\right)} \\
& \times \prod_{i=1}^{k}\left[\frac{\pi}{\sin \left(\pi\left(v_{i}+z_{i}\right)\right)} \frac{\overline{\mathcal{G}}\left(v_{i}\right)}{\overline{\mathcal{G}}\left(-z_{i}\right)} \frac{\Gamma\left(2 v_{i}\right)}{\Gamma\left(v_{i}-z_{i}\right)} \frac{u^{z_{i}+v_{i}}}{z_{i}+v_{i}}\right],
\end{aligned}
$$

where $R$ is chosen so that for all $i,-\alpha_{i}<R<\min \left\{0, \alpha_{\circ}, 1-\alpha_{i}\right\}$, the contours for each variable $v_{i}$ are positively oriented circles enclosing the poles $\left\{\alpha_{j}\right\}_{1 \leqslant j \leqslant n}$ 
and no other singularity of the integrand, and

$$
\overline{\mathcal{G}}(v)=\frac{e^{-\tau v^{2} / 2}}{\Gamma\left(\alpha_{\circ}+v\right)} \frac{\prod_{j=1}^{n} \Gamma\left(\alpha_{j}-v\right)}{\prod_{j=1}^{t} \Gamma\left(\alpha_{j}+v\right)} .
$$

An important feature of (5) is the cross product

$$
\prod_{1 \leqslant i<j \leqslant k} \frac{\Gamma\left(v_{i}+v_{j}\right) \Gamma\left(-z_{i}-z_{j}\right)}{\Gamma\left(v_{j}-z_{i}\right) \Gamma\left(v_{i}-z_{j}\right)} .
$$

If the Gamma functions were replaced by their rational approximation around zero, that is $\Gamma(z) \sim 1 / z$, we could recast the right-hand side of (5) as the Fredholm Pfaffian of an explicit kernel and the asymptotic analysis would become much easier. Unfortunately, this cross product grows with $k$ as $e^{c k^{2}}$, which makes it difficult to control the series (5) as the number of terms $n$ goes to infinity. Similar issues involving a cross product with Gamma factors have been encountered several times in exact formulas for models in the KPZ universality class, in particular in [OQR17, NZ16].

\subsubsection{Relation to the work of O'Connell-Seppäläinen-Zygouras. A model} equivalent to the half-space log-gamma polymer model was considered in [OSZ14]. It corresponds to a log-gamma polymer model where paths reside in the first quadrant, as in the usual log-gamma polymer, but the weights $\tilde{w}_{i, j}$ are symmetric with respect to the first diagonal $\left(\tilde{w}_{i, j}=\tilde{w}_{j, i}\right)$. Off-diagonal weights are distributed as $w_{i, j} \sim \operatorname{Gamma}^{-1}\left(\alpha_{i}+\alpha_{j}\right)$ while the diagonal weights are distributed as $w_{i, i} \sim \frac{1}{2}$ Gamma $^{-1}\left(\alpha_{i}+\alpha_{\circ}\right)$. One can identify the weight of a path in this model with the weight of a path in the half-space log-gamma polymer from Definition 1.1 up to a factor $(1 / 2)^{k}$, where $k$ is the number of times the path hits the diagonal. Since there are $2^{k-1}$ paths in the symmetrized model, which correspond to the same path in the half-space model, the partition function $\tilde{Z}(t, n)$ of the symmetrized model is such that $\widetilde{Z}(t, n)=\frac{1}{2} Z(t, n)$. When $t=n$, the law of $\widetilde{Z}(n, n)$ is a marginal of the push-forward of a symmetric matrix with inverse-Gamma random variables by the geometric RSK algorithm. [OSZ14] computed this push-forward (and hence the distribution of $\widetilde{Z}(n, n)$ ) as the Whittaker measure (with slightly different notations than in the present paper). By a formal (see Section 6.8) application of the Plancherel theorem for Whittaker functions, they derived a conjectural formula for the Laplace transform of $\widetilde{Z}(n, n)$ [OSZ14, (5.15), (5.16)]. Though [OSZ14] was unable to prove this formula, they suggested that 'it seems reasonable to expect the integral formulas (5.15) and (5.16) to be valid, at least in some suitably regularized sense'. In our present work, we show that our hybrid polymer provides such 
an appropriate regularization. The reason why this was inaccessible to [OSZ14] was that their results (and connection to Whittaker measures) were restricted to the diagonal and the hybrid polymer requires working off-diagonal, hence the interest of our study of Markov dynamics on half-space Macdonald processes. Using Whittaker Plancherel theory for our hybrid model and letting $\tau$ go to zero, we obtain the following formula.

For $t \geqslant n$ and any $u>0$, we have (see Corollary 6.40)

$$
\begin{aligned}
\mathbb{E}\left[e^{-u Z(t, n)}\right] & \frac{1}{n !} \int_{r-\mathbf{i} \infty}^{r+\mathbf{i} \infty} \frac{d z_{1}}{2 \mathbf{i} \pi} \cdots \int_{r-\mathbf{i} \infty}^{r+\mathbf{i} \infty} \frac{d z_{n}}{2 \mathbf{i} \pi} \prod_{i \neq j} \frac{1}{\Gamma\left(z_{i}-z_{j}\right)} \prod_{1 \leqslant i<j \leqslant n} \frac{\Gamma\left(z_{i}+z_{j}\right)}{\Gamma\left(\alpha_{i}+\alpha_{j}\right)} \\
& \times \prod_{i, j=1}^{n} \Gamma\left(z_{i}-\alpha_{j}\right) \prod_{i=1}^{n}\left(u^{\alpha_{i}-z_{i}} \frac{\Gamma\left(\alpha_{\circ}+z_{i}\right)}{\Gamma\left(\alpha_{\circ}+\alpha_{i}\right)} \prod_{j=n+1}^{t} \frac{\Gamma\left(\alpha_{j}+z_{i}\right)}{\Gamma\left(\alpha_{j}+\alpha_{i}\right)}\right),
\end{aligned}
$$

where $r>0$ is such that $r+\alpha_{\circ}>0$ and $r>\alpha_{i}$ for all $1 \leqslant i \leqslant n$. We show in Corollary 6.41 how to deduce rigorously [OSZ14, (5.15), (5.16)] from the above formula. Note that (6) is more general since we consider the partition function at any point $(t, n)$, not only when $t=n$.

Applying the geometric RSK algorithm to inverse-Gamma-distributed matrices with other types of symmetries was further considered in [BZ19], but the corresponding polymer models do not seem to be related to the present paper. Dynamics on Gelfand-Tsetlin patterns restricted by a wall were studied in Nteka's PhD thesis [Nte16], but it is not clear if this is related to our present paper.

1.3.3. Half-space stochastic six-vertex model. The stochastic six-vertex model was introduced in [GS92] and further studied in [BCG16]. It was related to (fullspace) Hall-Littlewood processes in [Bor18] (see also [BBW16, BM18]). Halfspace variants of the stochastic six-vertex model and half-space Hall-Littlewood processes were discussed in [BBCW18].

Definition 1.2. Consider the half-quadrant $\left\{(x, y) \in \mathbb{Z}_{>0}^{2}: x \leqslant y\right\}$. The stochastic six-vertex model in the half-quadrant is a probability measure on collections of up-right paths (see Figure 3). We associate with each vertex a Boltzmann weight determined by the local configuration of adjacent paths. In the bulk, for a vertex $(x, y)$ with $x>y$, there are six possible configurations and we choose the Boltzmann weights as

$$
\mathbb{P}(\vdots)=\frac{1-a_{x} a_{y}}{1-t a_{x} a_{y}}, \quad \mathbb{P}(-\ldots)=\frac{(1-t) a_{x} a_{y}}{1-t a_{x} a_{y}},
$$




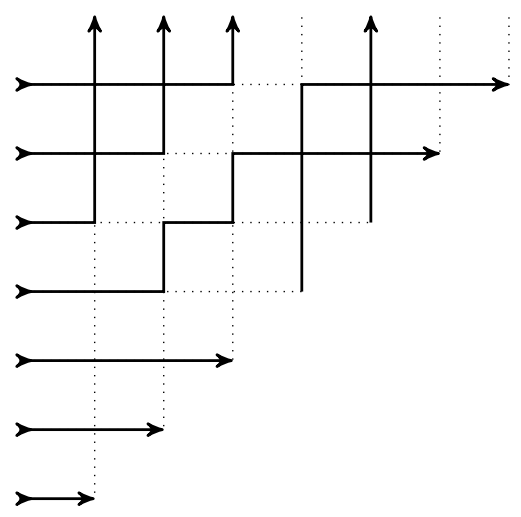

Figure 3. Sample configuration of the stochastic six-vertex model in a halfquadrant.

$$
\mathbb{P}(\cdots \mid \ldots)=\frac{t\left(1-a_{x} a_{y}\right)}{1-t a_{x} a_{y}}, \quad \mathbb{P}(\cdots,)=\frac{1-t}{1-t a_{x} a_{y}} .
$$

For a corner vertex of the form $(x, x)$, we choose weights as

$$
\mathbb{P}(\rightarrow)=\mathbb{P}(\ldots \downarrow)=1, \quad \mathbb{P}(\downarrow)=\mathbb{P}(\ldots)=0 .
$$

These weights are stochastic in the sense that

$$
\begin{aligned}
& \mathbb{P}(\lfloor)+\mathbb{P}(\downarrow)=\mathbb{P}(\lceil)+\mathbb{P}(\mid)=1, \\
& \mathbb{P}(\rightarrow)+\mathbb{P}(\downarrow)=\mathbb{P}(\downarrow)+\mathbb{P}\left(\begin{array}{l}
\vdots \\
\downarrow
\end{array}\right)=1 .
\end{aligned}
$$

We define a probability measure on configurations of up-right paths as follows. We assume that there is an incoming horizontal edge to each vertex $(1, y)$ on the left boundary. Assume that for some $n \geqslant 2$, the incoming edge states of the set of vertices $\{(x, y)\}_{x+y=n}$ are all determined. Choose the outgoing edge states of these vertices by sampling from the Bernoulli distribution imposed by the vertex weights above. This determines the incoming states of the set of vertices $\{(x, y)\}_{x+y=n+1}$, and iterating this procedure defines the probability distribution of configurations on the whole half-quadrant. This implies that the probability distribution of the restriction of the configuration to a finite set of vertices near the origin such as in Figure 3 is given by the product of Boltzmann weights. We refer the reader to [BBCW18, Section 3] for a more precise definition. 
Using the relation between half-space Hall-Littlewood measures and the stochastic six-vertex model established in [BBCW18] (see Theorem 5.7), we obtain moment formulas for the height function, stated as Corollary 5.8 in the text:

$$
\begin{aligned}
\mathbb{E}^{t}\left[t^{-k \mathfrak{h}(x, y)}\right]= & t^{\frac{k(k-1)}{2}} \oint_{C_{1}} \frac{d z_{1}}{2 \mathbf{i} \pi} \cdots \oint_{C_{k}} \frac{d z_{k}}{2 \mathbf{i} \pi} \prod_{1 \leqslant i<j \leqslant k} \frac{z_{i}-z_{j}}{z_{i}-t z_{j}} \frac{1-t z_{i} z_{j}}{1-z_{i} z_{j}} \\
& \times \prod_{j=1}^{k}\left(\frac{1}{z_{j}} \frac{1-t z_{j}^{2}}{1-z_{j}^{2}} \prod_{i=1}^{y} \frac{1-a_{i} z_{j}}{1-t a_{i} z_{j}} \prod_{i=1}^{x} \frac{z_{j}-a_{i} / t}{z_{j}-a_{i}}\right),
\end{aligned}
$$

where the contours $C_{1}, \ldots, C_{m}$ all enclose 0 and $a_{i}$ are contained in the open disk of radius 1 around zero, and the contours are nested in such a way that for $i<j$ the contour $C_{i}$ does not include any part of $t C_{j}$.

The half-space six-vertex model is a discrete-time version of the half-line ASEP (see Definition 5.9). The formula above is similar to nested contour integral formulas obtained in [BCS14] for the full-space ASEP using coordinate Bethe ansatz. It is likely that the formula above can be obtained through coordinate Bethe ansatz as well, and we plan to study this further in future work.

Owing to a refined Littlewood identity originally conjectured in [BWZJ15] and later proved in [Rai18], [BBCW18] determined-for a certain initial data and a specific boundary condition - the distribution of the height function at the boundary for ASEP and the KPZ equation in a half-space using a limit of the halfspace stochastic six-vertex model. Note that with the techniques of [BBCW18] is was possible to characterize the distribution of $\mathfrak{h}(x, y)$ only when $x=y$.

1.4. Asymptotics. We turn to the asymptotic results that can be derived (at least formally) from our formulas.

1.4.1. Log-gamma polymer. In Section 8, we perform an asymptotic analysis of our Laplace transform formula (5) as $n$ goes to infinity. We assume that the parameters $\alpha_{i}$ of the log-gamma polymer are all equal to some $\alpha>0$, and we keep the boundary parameter $\alpha_{\text {。 }}$ arbitrary (thus we have weights distributed as $\operatorname{Gamma}^{-1}\left(\alpha_{\circ}+\alpha\right)$ on the boundary and $\operatorname{Gamma}^{-1}(2 \alpha)$ in the bulk). A nonrigorous application of Laplace's method yields the following limit laws. (The digamma and polygamma functions are defined as $\Psi(z)=\frac{d}{d z} \log (\Gamma(z)$ ) and $\Psi_{n}(z)=\frac{d^{n}}{d z^{n}} \Psi(z)$.) When $\alpha_{\circ}>0$,

$$
\lim _{n \rightarrow \infty} \mathbb{P}\left(\frac{\log (Z(n, n))-f n}{\sigma n^{1 / 3}} \leqslant x\right)=F_{\mathrm{GSE}}(x),
$$


where the quenched free energy $f=-2 \Psi(\alpha)$ and $\sigma=\sqrt[3]{\Psi_{2}(\alpha)}$. When $\alpha_{\circ}=0$,

$$
\lim _{n \rightarrow \infty} \mathbb{P}\left(\frac{\log (Z(n, n))-f n}{\sigma n^{1 / 3}} \leqslant x\right)=F_{\mathrm{GOE}}(x),
$$

with the same free energy $f=-2 \Psi(\alpha)$ and $\sigma=\sqrt[3]{\Psi_{2}(\alpha)}$. When $\alpha_{\circ}<0$,

$$
\lim _{n \rightarrow \infty} \mathbb{P}\left(\frac{\log (Z(n, n))-f_{\alpha_{o}} n}{\sigma_{\alpha_{o}} n^{1 / 2}} \leqslant x\right)=\int_{-\infty}^{x} \frac{e^{-t^{2} / 2}}{\sqrt{2 \pi}} d t,
$$

where the free energy becomes $f_{\alpha_{\circ}}=-\Psi\left(\alpha-\alpha_{\circ}\right)-\Psi\left(\alpha+\alpha_{\circ}\right)$ and $\sigma_{\alpha}=$ $\sqrt{\Psi_{1}\left(\alpha+\alpha_{\circ}\right)-\Psi_{1}\left(\alpha-\alpha_{\circ}\right)}$. Furthermore, if we scale $\alpha_{\circ}$ close to the critical point as $\alpha_{\circ}=n^{-1 / 3} \sigma^{-1} \varpi, F_{\mathrm{GOE}}$ would be replaced in (8) by a crossover distribution $F(x ; \varpi)$ such that $F(x ; 0)=F_{\mathrm{GOE}}(x)$ and $\lim _{\varpi \rightarrow \infty} F(x ; \varpi)=$ $F_{\mathrm{GSE}}(x)$. It was introduced in [BR01b, Definition 4] in relation with asymptotics of half-space last-passage percolation with geometric weights (see also [FNR06, BBCS18b, BBCS18a, BBNV18]).

Let us make clear that unlike all results stated previously, Equations (7), (8) and (9) are not completely proved, our asymptotics are nonrigorous at the level of neglecting convergence of tails of series and only focusing on critical points. Making these rigorous constitutes a significant challenge.

As $\alpha$ goes to zero, the free energy of the (half-space) log-gamma polymer converges to the last-passage time in a model of last-passage percolation with exponential weights in a half-quadrant. This model was considered in [BBCS18b], where the analogues (as $\left.\alpha_{\circ}, \alpha \rightarrow 0\right)$ of the limit laws (7), (8) and (9) were proved.

It is reasonable to expect that when $\alpha_{\circ}+\alpha$ is close to zero, the boundary weights will be so large that their contribution to the free energy will dominate and fluctuations will be Gaussian on the $n^{1 / 2}$ scale. On the contrary, if $\alpha_{\circ} \gtrsim \alpha$ we expect that the effect of the boundary should be limited, and fluctuations should occur on the scale $n^{1 / 3}$ by KPZ universality. We explain in Section 8.1 how to predict the critical $\alpha_{\circ}$ between Gaussian and KPZ behavior. We also provide heuristic arguments to explain the expression of the constants $f, f_{\alpha_{\circ}}$ and $\sigma_{\alpha_{\circ}}$ in (7), (8) and (9).

1.4.2. KPZ equation limit regime. Among models in the $\mathrm{KPZ}$ universality class, a central object is the KPZ equation-a stochastic PDE, which reads as

$$
\partial_{t} h(t, x)=\frac{1}{2} \Delta h(t, x)+\frac{1}{2}\left[\partial_{x} h(t, x)\right]^{2}+\xi \quad t>0, x \in \mathbb{R},
$$

where $\xi$ is a Gaussian space-time white noise. This stochastic PDE plays an important role because many models with a parameter controlling the asymmetry 
or the temperature converge to the KPZ equation under a certain scaling [AKQ14, DT16, HQ18, DGP17]. This fact is generally referred to as weak universality. Exact formulas characterizing the distribution of ASEP or directed polymers yield, after appropriate scaling, information about the distribution of the solutions to (10). This approach has been successfully implemented in [ACQ11, SS10, CDR10, Dot10, BCFV15] to determine the one-point distribution of the KPZ equation on the line $\mathbb{R}$, starting from several types of initial data. Since we know that statistics in the KPZ class usually depend on the geometry, it is natural to ask how the distribution would change for the KPZ equation on another spatial domain, for instance a circle, a segment or a half-line. A partial answer is provided in [BBCW18] for the KPZ equation on $\mathbb{R}_{\geqslant 0}$ with Neumann-type boundary condition $\partial_{x} h(t, 0)=-1 / 2$.

As in the full-space case [BC14, BCFV15], our formulas for the halfspace log-gamma should give, in the appropriate scaling regime, distributional information about the KPZ equation on $\mathbb{R}_{\geqslant 0}$ with Neumann-type boundary condition $\partial_{x} h(t, 0)=A$ (or, in other terms, the free energy of the continuous directed polymer model with a pinning at the boundary). It is not clear how to rigorously take asymptotics of our Laplace transform formulas (5) and (6) in the appropriate scaling regime. However, we consider in Section 7 the limit of our moment formula (4) and we do recover moment formulas obtained in [BBC16] for the partition function of the half-space continuous directed polymer (see Corollary 7.1). (The approach in [BBC16] requires uniqueness of the system of ordinary differential equations defining the delta Bose gas in a half-space [BBC16, Definition 3.2], which has not been proved.) In particular, we relate the parameter $\alpha_{\circ}$ of the half-space log-gamma polymer with the parameter $A$ involved in the boundary condition for the KPZ equation (we simply have $\alpha_{\circ}=A+1 / 2$ with our scalings).

\subsection{Outline of the paper.}

Section 2 After providing some background on Macdonald symmetric functions, we define half-space Macdonald measures and processes. We also provide a general scheme to build dynamics on sequences of partitions preserving the class of half-space Macdonald processes.

Section 3 We use operators, in particular Macdonald difference operators and Noumi's $q$-integral operator, diagonalized by Macdonald symmetric functions, to produce integral formulas for moments and Laplace transforms of Macdonald measures for general $q, t$ parameters.

Section 4 We study in more detail the case $t=0, q \in(0,1)$, called half-space 
$q$-Whittaker process. We consider the degeneration of general formulas and study a particular class of dynamics related to $q$-deformations of the RSK algorithm, introduced in [MP17]. This allows us to relate the half-space $q$-Whittaker process to the distribution of certain $q$-deformed particle systems (Section 4.3).

Section 5 We study in more detail the case $q=0, t \in(0,1)$, called the HallLittlewood process. We provide moment formulas, which, using results from [BBCW18], relate to the stochastic six-vertex model in a halfquadrant.

Section 6 We consider the $q \rightarrow 1$ degeneration of the half-space $q$-Whittaker process, called the half-space Whittaker process. The $q \rightarrow 1$ degeneration of the dynamics studied in Section 4 gives rise to the half-space log-gamma polymer. We also consider the degeneration of our integral formulas and relate them to the log-gamma directed polymer partition function.

Section 7 We define the KPZ equation on the positive reals and consider the scaling of the log-gamma directed polymer at high temperature which should lead to the continuous directed polymer, whose free energy solves the KPZ equation. We show that under these scalings, our moment formulas coincide with moment formulas previously obtained for the continuous directed polymer in a half-space in [BBC16].

Section 8 We consider asymptotics of the free energy $\log (Z(n, n))$ of the halfspace log-gamma polymer. We first provide probabilistic heuristics to predict the constants arising in limit theorems, for different ranges of $\alpha_{\circ}$. Then, we explain how, for each possible range of $\alpha_{\circ}$, the TracyWidom GSE or GOE or the Gaussian Central Limit Theorem arises from the Laplace transform formula (5). These asymptotics are, however, nonrigorous.

\section{Half-space Macdonald processes}

After fixing some useful notations and providing background on symmetric functions, we define in this section half-space Macdonald processes and explain a general scheme to build Markov dynamics preserving the set of such measures. 
2.1. $\boldsymbol{q}$-analogues. Throughout the paper, we assume that $0 \leqslant q<1$. Recall the definition of the $q$-Pochhammer symbol

$$
(a ; q)_{n}=(1-a)(1-a q) \ldots\left(1-a q^{n-1}\right) \quad \text { and } \quad(a ; q)_{\infty}=\prod_{i=0}^{\infty}\left(1-a q^{i}\right) .
$$

When there is no ambiguity possible, we may write simply $(a)_{\infty}$ instead of $(a ; q)_{\infty}$. Since $\frac{1-q^{n}}{1-q}$ goes to $n$ as $q$ goes to 1 , it is natural to define the $q$-integer $[n]_{q}$, the $q$-factorial $k !_{q}$ and $q$-binomial coefficients $\left(\begin{array}{l}n \\ k\end{array}\right)_{q}$ as

$$
[n]_{q}=\frac{1-q^{n}}{1-q}, \quad k !_{q}=\frac{(q ; q)_{k}}{(1-q)^{k}}=[1]_{q} \ldots[k]_{q}, \quad\left(\begin{array}{l}
n \\
k
\end{array}\right)_{q}=\frac{(q ; q)_{n}}{(q ; q)_{k}(q ; q)_{n-k}} .
$$

The $q$-binomial theorem states that for $|z|<1$,

$$
\sum_{k=0}^{\infty} \frac{z^{k}(a ; q)_{k}}{(q ; q)_{k}}=\frac{(a z ; q)_{\infty}}{(z ; q)_{\infty}}
$$

The $q$-exponential function is defined as

$$
e_{q}(z)=\frac{1}{(z(1-q) ; q)_{\infty}} .
$$

The $q$-binomial theorem shows that $e_{q}(z)$ converges as $q$ goes to 1 to the usual exponential $e^{x}$ uniformly on any compact set in the complex plane. The $q$ Gamma function is defined by

$$
\Gamma_{q}(z)=\frac{(q ; q)_{\infty}(1-q)^{1-z}}{\left(q^{z} ; q\right)_{\infty}} .
$$

When $z$ is not a negative integer, $\Gamma_{q}(z)$ converges to $\Gamma(z)$ as $q$ goes to 1 . We refer the reader to [AAR99] for more details.

2.2. Background on Macdonald symmetric functions. For a more comprehensive overview on (Macdonald) symmetric functions, see [Mac95] or [BC14, Section 2.1].

2.2.1. Partitions and Gelfand-Tsetlin patterns. A partition $\lambda$ is a nonincreasing sequence of nonnegative integers $\lambda_{1} \geqslant \lambda_{2} \geqslant \cdots$. The length of $\lambda$ is the number of its nonzero parts and is denoted by $\ell(\lambda)$. The weight of $\lambda$ is denoted as $|\lambda|:=\sum_{i} \lambda_{i}$. If $|\lambda|=n$, one says that $\lambda$ partitions $n$ (notation 
$\lambda \vdash n)$. The transpose $\lambda^{\prime}$ of a partition is defined by $\lambda_{i}^{\prime}=\left|\left\{j: \lambda_{j} \geqslant i\right\}\right|$. Let $\mathbb{Y}$ be the set of all partitions and let $\mathbb{Y}_{k}$ be its subset of partitions of length $k$. We will generally use Greek letters like $\lambda, \mu, \kappa, v, \pi, \tau$ to represent partitions. The empty partition (such that $\lambda_{1}=0$ ) is denoted by $\varnothing$. We will denote by $m_{i}(\lambda)$ the multiplicity of the integer $i$ in the sequence $\lambda$, and sometimes use the notation $\lambda=1^{m_{1}} 2^{m_{2}} \ldots$

A partition can be identified with a Young diagram or with a particle configuration in which for each $i$, there is a corresponding particle at position $\lambda_{i}$ (see Figure 4). For a box $\square$ in a Young diagram, $\operatorname{leg}(\square)$ is equal to the number of boxes in the diagram below it (the leg length) and $\operatorname{arm}(\square)$ is equal to the number of boxes in the diagram to the right of it (the arm length). A partition is even if all $\lambda_{i}$ are even. We write $\mu \subseteq \lambda$ if $\mu_{i} \leqslant \lambda_{i}$ for all $i$ and call $\lambda / \mu$ a skew Young diagram.

A partition $\mu$ interlaces with $\lambda$ if for all $i, \lambda_{i} \geqslant \mu_{i} \geqslant \lambda_{i+1}$. In the language of Young diagrams, this means that $\lambda$ can be obtained from $\mu$ by adding a horizontal strip in which at most one box is added per column. We denote interlacing by $\mu \prec \lambda$. In terms of the particle representation, interlacing refers to the interlacing of the locations of the two sets of particles. See Figure 4 for illustrations of some of these definitions.

2.2.2. Symmetric functions. Symmetric functions are defined with respect to an infinite number of formal variables (we will generally use arguments like $x=\left(x_{1}, x_{2}, \ldots\right)$ or $y=\left(y_{1}, y_{2}, \ldots\right)$ although the order of variables does not matter, or simply leave off the argument of a symmetric function when it is not important). We denote the algebra of symmetric functions by Sym. It can be seen as a commutative algebra $\mathbb{R}\left[p_{1}, p_{2}, \ldots\right]$, where $p_{k}(x)=x_{1}^{k}+x_{2}^{k}+\cdots$ are the Newton power sum symmetric functions, and we refer the reader to [Mac95, I] or [BC14, Section 2.1.2] for more details.

The skew Macdonald $P(Q)$ functions $P_{\lambda / \mu}\left(Q_{\lambda / \mu}\right)$ (see [Mac95, Ch. VI]) are symmetric functions indexed by skew partitions $\lambda / \mu$ that have coefficients in $\mathbb{Q}(q, t)$, which is the space of rational functions in two auxiliary parameters $q, t$ (we will assume them to be in $[0,1)$ ). For $\lambda \in \mathbb{Y}$, define symmetric functions

$$
\mathcal{E}_{\lambda}=\sum_{\mu^{\prime} \in \mathbb{Y} \text { even }} b_{\mu}^{\mathrm{el}} Q_{\lambda / \mu},
$$

where 'el' stands for 'even leg', and $b_{\mu}^{\mathrm{el}} \in \mathbb{Q}(q, t)$ is given by

$$
b_{\mu}^{\mathrm{el}}=\prod_{\substack{\square \in \mu \\ \operatorname{leg}(\square) \text { even }}} b_{\mu}(\square), \quad b_{\mu}(\square)= \begin{cases}\frac{1-q^{a} t^{\ell+1}}{1-q^{a+1} t^{\ell}} & \square \in \mu \\ 1 & \square \notin \mu\end{cases}
$$


(i)

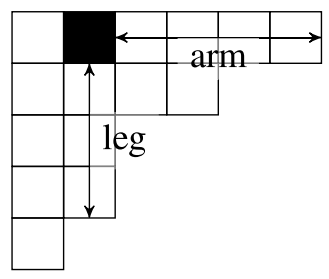

(ii)

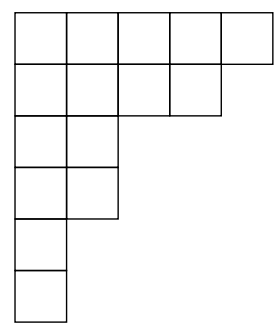

(iii)

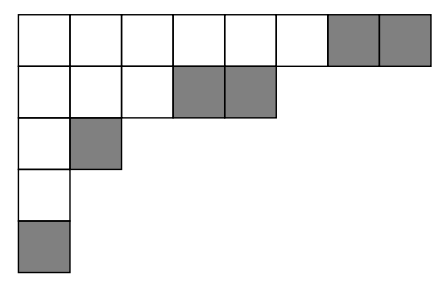

Figure 4. (i) Young diagram corresponding to the partition $\lambda=(6,4,2,2,1)$; the black box has arm length $a(\boldsymbol{\square})=4$ and leg length $\ell(\boldsymbol{\square})=3$. (ii) Young diagram corresponding to $\lambda$ 's transpose $\lambda^{\prime}=(5,4,2,2,1,1)$. (iii) The diagram contains a horizontal strip in gray added to the diagram $\mu=(6,3,1,1)$; the gray boxes are also the skew diagram $\kappa / \mu$, where $\kappa=(8,5,2,1,1)$.

with $\ell=\operatorname{leg}(\square)$ and $a=\operatorname{arm}(\square)$ in the definition of $b_{\mu}(\square)$ (see Figure 4 for the definitions of $\operatorname{leg}(\square)$ and $\operatorname{arm}(\square))$.

Macdonald symmetric functions satisfy the following combinatorial formula [Mac95, VI, (7.13)]. For two partitions $\lambda, \mu$ such that $\lambda / \mu$ is a horizontal strip, define coefficients

$$
\begin{aligned}
\varphi_{\lambda / \mu} & =\prod_{1 \leqslant i \leqslant j \leqslant \ell(\lambda)} \frac{f\left(q^{\lambda_{i}-\lambda_{j}} t^{j-i}\right) f\left(q^{\mu_{i}-\mu_{j+1}} t^{j-i}\right)}{f\left(q^{\lambda_{i}-\mu_{j}} t^{j-i}\right) f\left(q^{\mu_{i}-\lambda_{j+1}} t^{j-i}\right)}, \\
\psi_{\lambda / \mu} & =\prod_{1 \leqslant i \leqslant j \leqslant \ell(\mu)} \frac{f\left(q^{\mu_{i}-\mu_{j}} t^{j-i}\right) f\left(q^{\lambda_{i}-\lambda_{j+1}} t^{j-i}\right)}{f\left(q^{\lambda_{i}-\mu_{j}} t^{j-i}\right) f\left(q^{\mu_{i}-\lambda_{j+1}} t^{j-i}\right)},
\end{aligned}
$$

where $f(u)=(t u ; q)_{\infty} /(q u ; q)_{\infty}$. Then, we have that

$$
P_{\lambda / \mu}\left(x_{1}, \ldots, x_{n}\right)=\sum_{\lambda^{(1)}, \ldots, \lambda^{(n-1)}} \prod_{i=1}^{n} \psi_{\lambda^{(i)} / \lambda^{(i-1)}} x_{i}^{\left|\lambda^{(i)}\right|-\left|\lambda^{(i-1)}\right|},
$$

where the sum runs over sequences of partitions such that

$$
\mu=\lambda^{(0)} \subset \lambda^{(1)} \subset \cdots \subset \lambda^{(n)}=\lambda,
$$


where for all $1 \leqslant i \leqslant n, \lambda^{(i)} / \lambda^{(i-1)}$ is a horizontal strip. Similarly,

$$
Q_{\lambda / \mu}\left(x_{1}, \ldots, x_{n}\right)=\sum_{\lambda^{(1)}, \ldots, \lambda^{(n-1)}} \prod_{i=1}^{n} \varphi_{\lambda^{(i)} / \lambda^{(i-1)}} x_{i}^{\left|\lambda^{(i)}\right|-\left|\lambda^{(i-1)}\right|} .
$$

2.2.3. Identities. We recall certain identities involving symmetric functions, which will be utilized in the remainder of the paper. In this section, all summations run over the set $\mathbb{Y}$ of all partitions, unless otherwise specified.

The skew Cauchy identity [Mac95, VI.7] holds for two sets of formal variables $x$ and $y$ :

$$
\sum_{\kappa} P_{\kappa / \nu}(x) Q_{\kappa / \lambda}(y)=\Pi(x ; y) \sum_{\tau} Q_{\nu / \tau}(y) P_{\lambda / \tau}(x),
$$

where $\Pi(x ; y)$ is given by [Mac95, VI, (2.5)]

$$
\Pi(x ; y):=\sum_{\kappa} P_{\kappa}(x) Q_{\kappa}(y)=\prod_{i, j \geqslant 1} \phi\left(x_{i} y_{j}\right) \quad \text { where } \phi(x)=\frac{(t x ; q)_{\infty}}{(x ; q)_{\infty}}
$$

Macdonald $P$ and $Q$ functions also satisfy a sort of semigroup property called branching rule whereby [Mac95, VI.7]

$$
\sum_{\mu} P_{\nu / \mu}(x) P_{\mu / \lambda}(y)=P_{\nu / \lambda}(x, y) \quad \text { and } \quad \sum_{\mu} Q_{\nu / \mu}(x) Q_{\mu / \lambda}(y)=Q_{\nu / \lambda}(x, y) \text {. }
$$

Turning to the $\mathcal{E}_{\lambda}$ function, from [Mac95, VI.7, Ex. 4(i)] we have

$$
\Phi(x):=\sum_{v^{\prime} \in \mathbb{Y} \text { even }} b_{v}^{\mathrm{el}} P_{v}(x)=\prod_{i<j} \phi\left(x_{i} x_{j}\right)
$$

It follows from the definition of $\mathcal{E}_{\mu}$ along with (23) that

$$
\sum_{\mu} Q_{\lambda / \mu}(x) \mathcal{E}_{\mu}(y)=\mathcal{E}_{\lambda}(x, y) .
$$

From there, one can show (see for example [BBCW18, Proposition 2.1]) that

$$
\mathcal{E}_{\mu}(x)=\Phi(x)^{-1} \sum_{v^{\prime} \in \mathbb{Y} \text { even }} b_{v}^{\mathrm{el}} P_{v / \mu}(x) .
$$

Combining (21) with (26) yields

$$
\sum_{\mu} \mathcal{E}_{\mu}(x) P_{\mu / \lambda}(y)=\Pi(x ; y) \Phi(y) \mathcal{E}_{\lambda}(x, y),
$$


and in particular

$$
\sum_{\mu} \mathcal{E}_{\mu}(x) P_{\mu}(y)=\Pi(x ; y) \Phi(y)
$$

In the following, we will refer to (28) as the generalized Littlewood identity.

2.2.4. Specializations. A specialization $\rho$ of $\mathbf{S y m}$ is an algebra homomorphism of Sym to $\mathbb{C}$-see [BC14, Section 2.2.1] for a more involved discussion. We denote the application of $\rho$ to $f \in \mathbf{S y m}$ as $f(\rho)$. The trivial specialization $\rho=\varnothing$ takes the value 1 for the constant function $1 \in \mathbf{S y m}$ and 0 for all homogeneous functions $f \in \mathbf{S y m}$ of higher degree. The union of two specializations $\rho_{1}, \rho_{2}$ is defined via the relation

$$
p_{k}\left(\rho_{1}, \rho_{2}\right)=p_{k}\left(\rho_{1}\right)+p_{k}\left(\rho_{2}\right) \text {. }
$$

Since the power sums $p_{k}$ span Sym (algebraically), one can extend the definition of the union to any symmetric function. Notationally, we will write the union of $\rho_{1}, \rho_{2}$ by putting a comma between them.

We say a specialization $\rho$ is Macdonald nonnegative if for every skew diagram $\lambda / \mu, P_{\lambda / \mu}(\rho) \geqslant 0$. For nonnegative numbers $\alpha=\{\alpha\}_{i \geqslant 1}, \beta=\left\{\beta_{i}\right\}_{i \geqslant 1}$ and $\gamma$ such that $\sum_{i} \alpha_{i}+\beta_{i}<\infty$, we define the specialization $\rho=\rho(\alpha, \beta, \gamma)$ by

$$
\Pi(u ; \rho)=\sum_{n \geqslant 0} u^{n} Q_{(n)}(\rho)=\exp (\gamma u) \prod_{i \geqslant 1} \frac{\left(t \alpha_{i} u ; q\right)_{\infty}}{\left(\alpha_{i} u ; q\right)_{\infty}}\left(1+\beta_{i} u\right) .
$$

It has recently been proved in [Mat19, Theorem 1.4] that a specialization $\rho$ is Macdonald nonnegative if and only if $\rho=\rho(\alpha, \beta, \gamma)$ for some $\alpha, \beta, \gamma$ as above (the if part is not hard to show; the only if part was conjectured by Kerov in 1992). Note that we have used the same notation as $\Pi(x ; y)$, where $x$ is specialized into a single variable $u$ and $y$ is specialized into $\rho$. The $\alpha_{i}$ variables are called usual (because they correspond to the usual notion of evaluation of a polynomial into some variables), the $\beta_{i}$ are called dual and the $\gamma$ is called Plancherel. When $\rho$ only involves usual variables $\alpha_{1}, \ldots, \alpha_{k}$ (and all other $\alpha, \beta, \gamma$ are zero), $P_{\lambda}$ is supported on partitions $\lambda$ of $\ell(\lambda) \leqslant k$ and is a polynomial in the variables $\alpha_{i}$; when $\rho$ only involves dual variables $\beta_{1}, \ldots, \beta_{k}$ (and all other $\alpha, \beta, \gamma$ are zero), $P_{\lambda}$ is supported on partitions $\lambda$ of $\ell\left(\lambda^{\prime}\right) \leqslant k$ (in other words, all $\lambda_{i} \leqslant k$ ).

Specializations of Sym allow us to turn the formal summation identities of Section 2.2.3 into analytic ones. In particular, if there exist $0<R<1$ and specializations $\rho_{1}, \rho_{2}$ such that for all $k \geqslant 1$,

$$
\left|p_{k}\left(\rho_{1}\right)\right|<R^{k} \text { and }\left|p_{k}\left(\rho_{1}\right) p_{k}\left(\rho_{2}\right)\right|<R^{k},
$$


then the formal identity (28) becomes, after specializing $x$ into $\rho_{2}$ and $y$ into $\rho_{1}$,

$$
\sum_{\lambda \in \mathbb{Y}} \mathcal{E}_{\lambda}\left(\rho_{2}\right) P_{\lambda}\left(\rho_{1}\right)=\Pi\left(\rho_{1}, \rho_{2}\right) \Phi\left(\rho_{1}\right),
$$

where the sum is absolutely convergent.

2.2.5. Orthogonality. Macdonald symmetric functions $P_{\lambda}$ and $Q_{\lambda}$ form a basis of Sym, and they are orthogonal with respect to the scalar product $\langle\cdot, \cdot\rangle_{q, t}$ defined by

$$
\left\langle p_{\mu}, p_{\lambda}\right\rangle_{q, t}=\mathbf{1}_{\lambda=\mu} \prod_{i} i^{m_{i}(\lambda)} m_{i}(\lambda) ! \prod_{i=1}^{\ell(\lambda)} \frac{1-q^{\lambda_{i}}}{1-t^{\lambda_{i}}} .
$$

When specialized into $n$ usual variables, Macdonald symmetric functions are polynomials in these variables, and they are orthogonal with respect to another scalar product, introduced in [Mac95, VI, (9.10)]. In [BC14, Section 2.1.5], it is written as

$$
\langle f, g\rangle^{\prime}=\int_{\mathbb{T}^{n}} f(z) \overline{g(z)} \mathfrak{m}_{n}^{q, t}(z) \prod_{i=1}^{n} \frac{d z_{i}}{z_{i}}, \quad \mathfrak{m}_{n}^{q, t}(z):=\frac{1}{(2 \mathbf{i} \pi)^{n} n !} \prod_{i \neq j=1}^{n} \frac{\left(z_{i} / z_{j} ; q\right)_{\infty}}{\left(t z_{i} / z_{j} ; q\right)_{\infty}},
$$

where $\mathbb{T}^{n}$ is the $n$-fold torus $\left(\left\{e^{2 \mathbf{i} \pi \theta}\right\}_{\theta \in[0,1)}\right)^{n}$. Using the identity (22), we may write

$$
Q_{\lambda}(x)=\frac{\left\langle\Pi(\cdot, x), P_{\lambda}(\cdot)\right\rangle^{\prime}}{\left\langle P_{\lambda}, P_{\lambda}\right\rangle^{\prime}} .
$$

Similarly (28) would suggest

$$
\mathcal{E}_{\lambda}(x) \stackrel{?}{=} \frac{\left\langle\Pi(\cdot, x) \Phi(\cdot), P_{\lambda}(\cdot)\right\rangle^{\prime}}{\left\langle P_{\lambda}, P_{\lambda}\right\rangle^{\prime}},
$$

but this does not make sense because $\Phi$ has singularities on the torus, and (28) is not valid with such arguments. Thus, we rewrite the scalar product as

$$
\left\langle\langle f, g\rangle=\int_{\mathbb{T}^{n}} f(z) g\left(z^{-1}\right) \mathfrak{m}_{n}^{q, t}(z) \prod_{i=1}^{n} \frac{d z_{i}}{z_{i}},\right.
$$

where $z^{-1}=\left(1 / z_{1}, \ldots, 1 / z_{n}\right)$. (This is actually the original definition in [Mac95, VI.9].) Since for $w \in \mathbb{T}, \bar{w}=1 / w$, the scalar products $\langle\cdot, \cdot\rangle^{\prime}$ and $\langle\langle\cdot, \cdot\rangle\rangle$ coincide on polynomials, and Macdonald symmetric polynomials are orthogonal with respect to $\langle\langle\cdot, \cdot\rangle\rangle$ as well, with the same norm. In (31), the integrand is analytic as long as $f$ and $g$ are, so that we may use Cauchy's theorem and deform the 
contour. It is particularly convenient for us to take the contour as $c \mathbb{T}$, where $0<c<1$. We obtain using (28) that

$$
\mathcal{E}_{\lambda}(x)=\frac{1}{\left\langle\left\langle P_{\lambda}, P_{\lambda}\right\rangle\right\rangle} \int_{(c \mathbb{T})^{n}} P_{\lambda}\left(z^{-1}\right) \Pi(z, x) \Phi(z) \mathfrak{m}_{n}^{q, t}(z) \prod_{i=1}^{n} \frac{d z_{i}}{z_{i}} .
$$

REMARK 2.1. A very similar scalar product $\langle\langle\cdot, \cdot\rangle\rangle$ appears in [BCPS15a, BCPS15b, Bor17, BP18]. More precisely, taking $s=0$ in [Bor17, Theorem 7.2], we recover the orthogonality of Hall-Littlewood polynomials with respect to $\langle\langle\cdot, \cdot\rangle\rangle$.

2.3. Definition of half-space Macdonald processes. We define half-space Macdonald processes in terms of a certain type of paths in the sector $\{(i$, $\left.j) \in \mathbb{Z}^{2}: 0 \leqslant j \leqslant i\right\}$ decorated with Macdonald nonnegative specializations. An analogous definition of Pfaffian Schur processes-which are a particular case $q=t$ of the following-was described in [BBCS18b, Section 3.2]. An alternative but equivalent definition of half-space Macdonald processes was provided in [BBCW18, Definition 2.3]. The paths we consider are halfinfinite and oriented, starting at $(+\infty, 0)$ and proceeding to some $(i, 0)$ before proceeding by unit steps along upward and leftward edges until the diagonal, at which point there is a final edge connecting that point to $(0,0)$ where the path terminates. We will call such a path $\omega$ and denote its set of vertices as $V(\omega)$ and edges as $E(\omega)$. Denote the set of vertical edges by $E^{\uparrow}(\omega)$, of horizontal edges by $E^{\leftarrow}(\omega)$ (we do not include edges along the $x$-axis) and the singleton containing the diagonal edge by $E^{\swarrow}(\omega)$. The set of all such paths will be denoted by $\Omega$. Note that the last diagonal edge is a single edge, not a union of all of the $\sqrt{2}$ length edges between consecutive diagonal lattice points. Likewise, $V(\omega)$ does not include these intermediate diagonal points. We introduce a natural ordering on vertices: $v<v^{\prime}$ if $v$ comes before $v^{\prime}$ in $\omega$; likewise define similar precedence ordering on edges as well as between vertices and edges.

We label each edge $e \in E(\omega)$ with a Macdonald specialization $\rho_{e}$. We label each vertex $v \in V(\omega)$ by a partition $\lambda^{v}$ with the convention that $\lambda^{v} \equiv \varnothing$ for all $v$ with $y$-coordinate equal to 0 . Figure 5 provides an example of one such path (the $\varnothing$ vertices have been labeled, but all other vertices and edge labels are not present in the figure).

For a given path $\omega \in \Omega$ and set of specializations $\rho=\left\{\rho_{e}\right\}_{e \in E(\omega)}$, we associate a weight to the sequence of partitions $\lambda=\left\{\lambda^{v}\right\}_{v \in V(\omega)}$ :

$$
\mathcal{W}(\lambda):=\prod_{e \in E(\omega)} \mathcal{W}(e)
$$




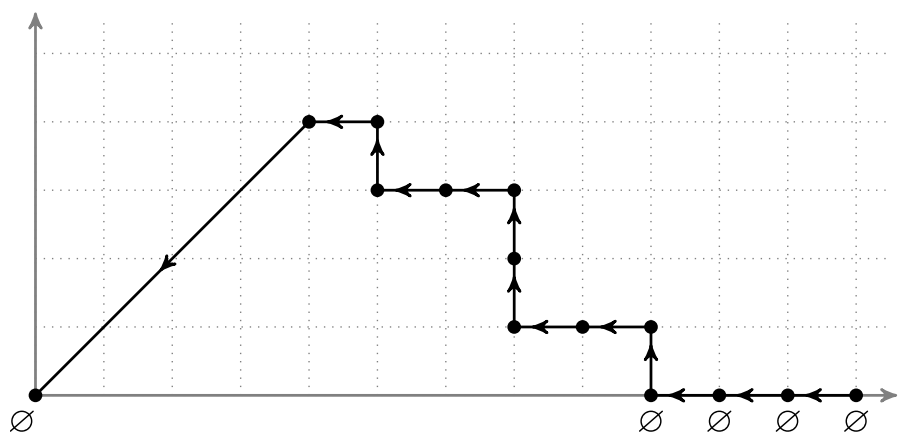

Figure 5. A possible path $\omega \in \Omega$. The vertices with $y$-coordinate 0 are labeled with trivial partitions, while all others are (not shown) labeled with partitions and all edges are labeled with Macdonald nonnegative specializations.

where the weight of an edge $e$ is given as follows. Let $\kappa$ denote the partition at the start of $e$ and $\mu$ the partition at the end of $e$. Then,

$$
\mathcal{W}(e)= \begin{cases}\mathcal{E}_{\kappa}\left(\rho_{e}\right) & \text { if } e \in E^{\swarrow}(\omega), \\ Q_{\kappa / \mu}\left(\rho_{e}\right) & \text { if } e \in E^{\leftarrow}(\omega), \\ P_{\mu / \kappa}\left(\rho_{e}\right) & \text { if } e \in E^{\uparrow}(\omega) .\end{cases}
$$

We use the convention $P_{\varnothing / \varnothing}=1$ so that if $e$ is a leftward edge with $y$-coordinate 0 , then $\mathcal{W}(e)=1$.

PROPOSITION 2.2. Assuming each term on the right-hand side is finite,

$$
\sum_{\lambda} \mathcal{W}(\lambda)=\prod_{\substack{e<e^{\prime}: \\ e \in E^{\uparrow}(\omega), e^{\prime} \in E^{\leftarrow}(\omega) \cup E^{\prime}(\omega)}} \Pi\left(\rho_{e} ; \rho_{e^{\prime}}\right) \Phi\left(\cup_{e \in E^{\uparrow}(\omega)} \rho_{e}\right) .
$$

Proof. This can be proved through applying (specializations of) identities (21), (23), (25) and (27). We provide a pictorial proof that explains in which order these identities must be used. Figure 6 provides a graphical representation for the meaning of each identity.

Starting from any path $\omega$, one may apply these elementary moves until $\omega$ is reduced to the trivial path (with $x$-coordinate always equal to zero), which assigns weight 1 to trivial partitions and 0 otherwise. Figure 7 provides a stepby-step illustration of this reduction process. Keeping track of the products of $\Pi$ and $\Phi$ terms yields the desired formula. 
(a)

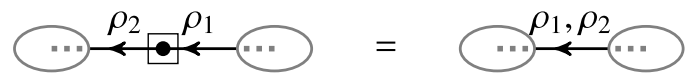

(b)
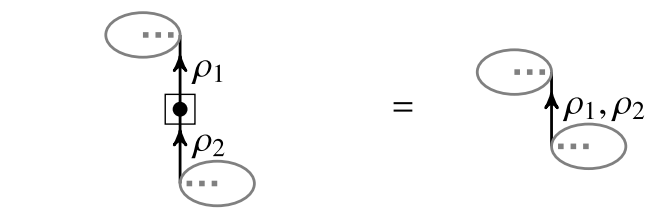

(c)
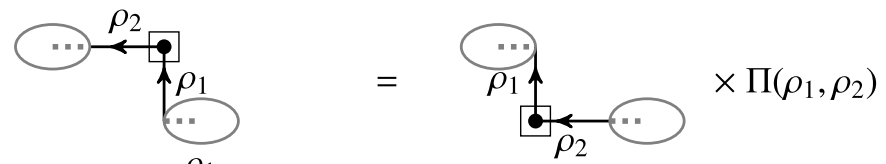

(d)
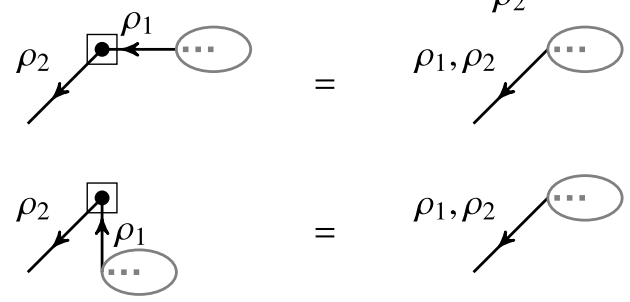

$=\rho_{1}, \rho_{2}$

$\times \Pi\left(\rho_{1}, \rho_{2}\right) \Phi\left(\rho_{1}\right)$

Figure 6. Graphical representations of summation identities. The boxes represent vertices whose partitions are being summed over; the directed edges are labeled by Macdonald nonnegative specializations; the blobs represent other terms that may arise in the weight of a path $\omega$, which are not involved in these identities. Graphics (a) represents the branching rule (23) for Macdonald polynomials $Q$, (b) represents the branching rule for polynomials $P$, (c) represents the skew Cauchy identity (21), (d) represents (25) and (e) represents (27).

DEFinition 2.3. For a given path $\omega \in \Omega$ and a set of Macdonald nonnegative specializations $\boldsymbol{\rho}=\left\{\rho_{e}\right\}_{e \in E(\omega)}$ such that (34) is finite, the half-space Macdonald process $\mathbb{P}_{\omega ; \rho}^{q, t}$ is a measure on the sequence of partitions $\lambda=\left\{\lambda^{v}\right\}_{v \in V(\omega)}$ given by

$$
\mathbb{P}_{\omega ; \boldsymbol{\rho}}^{q, t}(\boldsymbol{\lambda}):=\frac{\mathcal{W}(\boldsymbol{\lambda})}{\prod_{\substack{e<e^{\prime}: \\ e \in E^{\uparrow}(\omega), e^{\prime} \in E^{\leftarrow}(\omega) \cup E^{\swarrow}(\omega)}} \Pi\left(\rho_{e} ; \rho_{e^{\prime}}\right) \Phi\left(\bigcup_{e \in E^{\uparrow}(\omega)} \rho_{e}\right)} .
$$

The half-space Macdonald measure is a measure on a single partition. It is a special case of the process when the path $\omega=\Lambda$, that is, the path with only one vertex above the $x$-axis at position $(1,1)$. In that case, the only nontrivial partition is $\lambda^{(1,1)}$ (which we will just write as $\lambda$ ) and the only specializations that matter are those for the upward edge into $(1,1)$, which we denote by $\rho$, and 

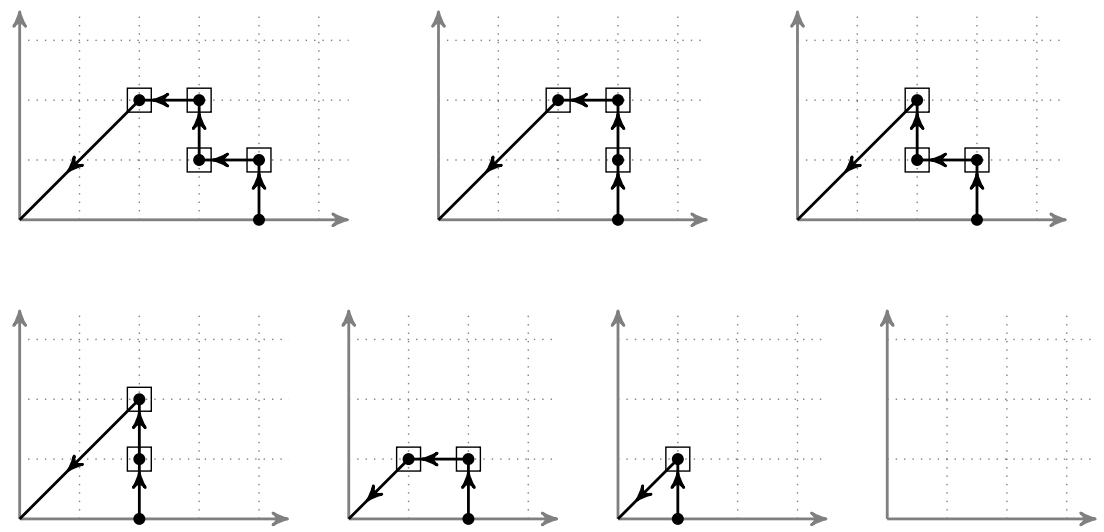

Figure 7. To compute the normalizing constant for a path $\omega$, one sums over all nontrivial partitions labeled by vertices. The boxes represent these summations, and the figure shows the sequential application of the identities in Figure 6 . The multiplicative factors of $\Pi$ and $\Phi$, which arise from each summation are not shown nor are the specializations.

that of the diagonal edge out of $(1,1)$, which we denote by $\rho^{\swarrow}$. The half-space Macdonald measure is then simply written as $\mathbb{P}_{\rho, \rho}^{q, t}$ and is explicitly given by the formula

$$
\mathbb{P}_{\rho, \rho \swarrow}^{q, t}(\lambda)=\frac{P_{\lambda}(\rho) \mathcal{E}_{\lambda}\left(\rho^{\swarrow}\right)}{\Pi\left(\rho ; \rho^{\swarrow}\right) \Phi(\rho)} .
$$

It is convenient to also introduce an expectation operator, which for the halfspace Macdonald process is denoted by $\mathbb{E}_{\omega ; \rho}^{q, t}$ and for the measure is denoted by $\mathbb{E}_{\rho, \rho \downarrow}^{q, t}$.

It is easy to show using the relations in Figure 6 that various marginals of halfspace Macdonald processes to subsequences of $\lambda$ remain half-space Macdonald processes. In particular, for a particular vertex $v \in \omega$, the marginal distribution of $\lambda^{v}$ under the half-space Macdonald process $\mathbb{P}_{\omega ; \rho}^{q, t}$ is given by the half-space Macdonald measure $\mathbb{P}_{\rho, \rho}^{q, t}(\lambda)$, where

$$
\rho=\bigcup_{e<v: e \in E^{\uparrow}(\omega)} \rho_{e} \quad \text { and } \quad \rho^{\swarrow}=\bigcup_{e>v: e \in E(\omega)} \rho_{e} .
$$

REMARK 2.4. (Full-space) Macdonald processes [BC14] can be defined in a similar manner. The difference is that the paths $\omega$ that index Macdonald 
processes start at $(+\infty, 0)$ and end at $(0,+\infty)$. The weight of a collection of partitions on vertices of the path is still given by the product of specialized skew Macdonald $P$ or $Q$ functions along the edges.

REMARK 2.5. Macdonald (and in particular, Schur) processes are commonly defined as measures on sequences $\lambda=\left(\lambda^{(1)}, \ldots, \lambda^{(N)}\right)$ and $\mu=\left(\mu^{(1)}, \ldots\right.$, $\left.\mu^{(N-1)}\right)$ of partitions satisfying the interlacing condition

$$
\varnothing \subset \lambda^{(1)} \supset \mu^{(1)} \subset \lambda^{(2)} \supset \mu^{(2)} \subset \cdots \supset \mu^{(N-1)} \subset \lambda^{(N)} \supset \varnothing .
$$

In the context of half-space Macdonald processes, we could similarly define our measure on such a set of partitions by fixing Macdonald nonnegative specializations $\rho_{0}^{+}, \ldots \rho_{N-1}^{+}, \rho_{1}^{-}, \ldots, \rho_{N}^{-}$and defining a weight

$\mathcal{W}(\boldsymbol{\lambda}, \boldsymbol{\mu}):=P_{\lambda^{(1)}}\left(\rho_{0}^{+}\right) Q_{\lambda^{(1)} / \mu^{(1)}}\left(\rho_{1}^{-}\right) P_{\lambda^{(2)} / \mu^{(1)}}\left(\rho_{1}^{+}\right) \cdots P_{\lambda^{(N)} / \mu^{(N-1)}}\left(\rho_{N-1}^{+}\right) \mathcal{E}_{\lambda^{(N)}}\left(\rho_{N}^{-}\right)$.

This was the definition employed in [BBCW18, Definition 3.2] and [BR05] in the Schur case. It is easy to match this measure to a half-space Macdonald process indexed by a particular choice of path $\omega$ (which maximally zigzags from the $x$-axis to the diagonal). More general choices of $\omega$ come from choosing trivial specializations (which force equality of consecutive partitions). Thus Definition 2.3 is equivalent to [BBCW18, Definition 3.2]-see also [BBCS18b, Remark 3.6] about the equivalence between both formulations.

The next proposition is a useful identity in law valid only when the diagonal specialization is the evaluation into a single variable (see Section 8.1 for an application). In the Schur degeneration, one recovers [BR01a, Corollary 7.6] (see also [BBCS18b, Proposition 3.4]).

Proposition 2.6. Let $\mu$ be distributed according to the Macdonald measure $\mathbb{P}_{\rho, \alpha}^{q, t}$ where $\alpha$ is a single variable specialization, and let $\lambda$ be distributed according to the Macdonald measure $\mathbb{P}_{\rho^{\prime}, 0}^{q, t}$ with $\rho^{\prime}=(\rho, \alpha)$. Then $\left(\lambda_{1}, \lambda_{3}, \ldots\right)$ and $\left(\mu_{1}, \mu_{3}, \ldots\right)$ have the same distribution.

Proof. Since $\mathcal{E}_{\lambda}(0)$ is supported on partitions with even dual,

$$
\mathbb{P}_{\rho^{\prime}, 0}^{q, t}\left(\lambda_{1} \leqslant \ell_{1}, \lambda_{3} \leqslant \ell_{3}, \ldots\right)=\sum_{\lambda^{\prime} \text { even }} b_{\lambda}^{\mathrm{el}} P_{\lambda}\left(\rho^{\prime}\right)
$$

where the sum runs over partitions $\lambda$ such that $\lambda_{i} \leqslant \ell_{i}$ for all odd $i$. Using (23), the sum can be rewritten as

$$
\sum_{\lambda^{\prime} \text { even }} \sum_{\mu \subset \lambda} b_{\lambda}^{\mathrm{el}} P_{\lambda / \mu}(\alpha) P_{\mu}(\rho)=\sum_{\mu<\lambda} \sum_{\lambda^{\prime} \text { even }} b_{\lambda}^{\mathrm{el}} \psi_{\lambda / \mu} \alpha^{|\lambda-\mu|} P_{\mu}(\rho),
$$


where on the right-hand side the first sum runs over partitions $\mu$ such that $\mu_{i} \leqslant \ell_{i}$ for all odd $i$. Now we will use the fact that if $\mu \prec \lambda$ and $\lambda^{\prime}$ is even, then we have (see [Mac95, VI.7 Ex. 4, Eq. (4)])

$$
b_{\lambda}^{\mathrm{el}} \psi_{\lambda / \mu}=b_{v}^{\mathrm{el}} \varphi_{\mu / v},
$$

where $v$ is the only partition such that $v \prec \mu$ and $v^{\prime}$ is even, and the coefficients $\psi_{\lambda / \mu}, \varphi_{\mu / \nu}$ are defined in (18). Since we also have $|\lambda-\mu|=|\mu-\nu|$, (35) equals

$$
\sum_{\mu} b_{\nu}^{\mathrm{el}} \varphi_{\mu / \nu} \alpha^{|\mu-\lambda|} P_{\mu}(\rho)
$$

We recognize $\mathcal{E}_{\mu}(\alpha)=b_{\nu}^{\mathrm{el}} \varphi_{\mu / \nu} \alpha^{|\mu-\lambda|}$ and conclude that

$$
\mathbb{P}_{\rho^{\prime}, 0}^{q, t}\left(\lambda_{1} \leqslant \ell_{1}, \lambda_{3} \leqslant \ell_{3}, \ldots\right)=\mathbb{P}_{\rho, \alpha}^{q, t}\left(\mu_{1} \leqslant \ell_{1}, \mu_{3} \leqslant \ell_{3}, \ldots\right) .
$$

\subsection{Markov dynamics on half-space Macdonald processes.}

2.4.1. Bulk and boundary transition operators. We consider here Markov transition operators that map half-space Macdonald processes with one set of parameters to those processes with an updated set of parameters. We will leverage the graphical representation of the half-space Macdonald process so as to describe a general mechanism through which to 'grow' such a measure. We refer to [BBCS18b, Section 3.3], where such a procedure is explained in the case of the Pfaffian Schur processes. In terms of the path $\omega$, there are two elementary moves (see Figure 8), which can be used to transition between any $\omega$ and $\omega^{\prime}$ where $\omega^{\prime}$ contains $\omega$ (in the sense that $\omega$ sits entirely to the bottom left of $\left.\omega^{\prime}\right)$. The first move — bulk growth—takes a piece $\llcorner$ in $\omega$ with corner coordinates $(i, j)$ with $i>j$ and inverts it into piece $\urcorner$ with corner coordinates $(i+1$, $j+1)$. The second move-boundary growth-takes the piece composed of the diagonal from $(i, i)$ to $(0,0)$ and the leftward edge immediately preceding it and replaces it with the diagonal from $(i+1, i+1)$ to $(0,0)$ and an upward edge immediately preceding it. To each type of moves, we associate an operator. For the bulk growth, out of corner $(i, j)$ with $i>j$, we associate the operator $\mathcal{U}_{i, j}^{\llcorner}$ and for the boundary growth, out of corner $(i, i)$ we associate the operator $\mathcal{U}_{i, i}^{\perp}$.

The specializations will be carried out from the initial path to the new one as follows. The operator $\mathcal{U}_{i, j}^{\llcorner}$will encode a Markov transition from $\lambda^{(i, j)}$ to $\lambda^{(i+1, j+1)}$ and will be chosen so as to map the half-space Macdonald process to a new half-space Macdonald process on the new path $\omega^{\prime}$ where the specializations all remain the same, except the leftward one into $(i, j)$ becomes the leftward one out of $(i+1, j+1)$ and likewise the upward one out of $(i, j)$ becomes the 


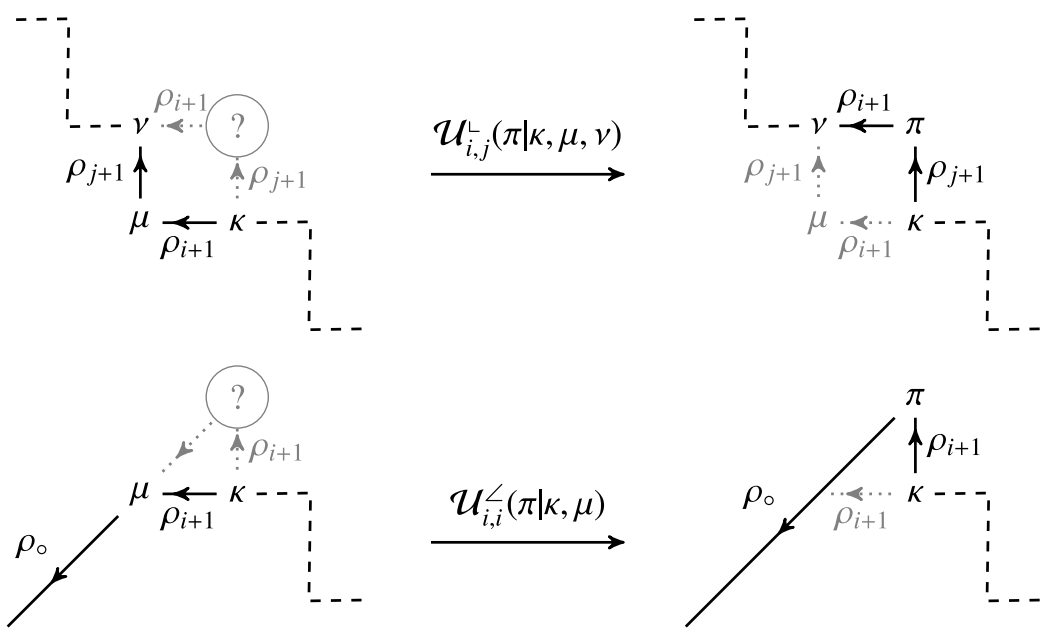

Figure 8. Operators $\mathcal{U}_{(i, j)}^{\llcorner}$and $\mathcal{U}_{(i, i)}^{\llcorner}$map a half-space Macdonald process to a new half-space Macdonald process indexed by a new path with specializations chosen as above. The dashed part of the path does not play any role.

upward one into $(i+1, j+1)$. The operator $\mathcal{U}_{i, i}^{L}$ will encode a Markov transition from $\lambda^{(i, i)}$ to $\lambda^{(i+1, i+1)}$ and should map the half-space Macdonald process to a new half-space Macdonald process on the new path $\omega^{\prime}$ where the specializations all remain the same (including the diagonal edge from $(i+1, i+1)$ to $(0,0))$, except the leftward one into $(i, i)$ becomes the upward one into $(i+1, i+1)$.

Our use of the operator $\mathcal{U}^{\llcorner}$implies that we use the same specializations on all vertical edges at the same ordinate and on all horizontal edges at the same abscissae. Let us denote by $\rho_{i}^{\mathrm{h}}$ the specialization carried by any horizontal edge between a point $(i-1, j)$ to a point $(i, j)$, and $\rho_{j}^{\mathrm{v}}$ the specialization carried by any vertical edge between a point $(i, j-1)$ to a point $(i, j)$. Moreover, our use of the operator $\mathcal{U}^{L}$ implies that for every $i \in \mathbb{Z}_{\geqslant 0}, \rho_{i}^{\mathrm{h}}=\rho_{i}^{\mathrm{v}}$. We will henceforth use the notation $\rho_{i}:=\rho_{i}^{\mathrm{h}}=\rho_{i}^{\mathrm{v}}$. We will also denote by $\rho_{\circ}$ the specialization on the diagonal edge.

Note that despite this seemingly restrictive choice of specializations, any halfspace Macdonald process - defined by any admissible path $\omega \in \Omega$ and arbitrary specializations on $E(\omega)$ - can be realized as the output of the above transition operators along a sequence of elementary moves from the empty path to the path $\omega$.

There may be many ways to choose the operators $\mathcal{U}^{\llcorner}$and $\mathcal{U}^{\perp}$. We will restrict our attention to those $\mathcal{U}_{(i, j)}^{\llcorner}$that only depend on the partitions at the three vertices 
$(i, j+1),(i, j),(i+1, j)$ as well as the two specializations between these vertices, and those $\mathcal{U}_{i}^{L}$ that only depend on the two vertices at $(i, i)$ and $(i+1, i)$ as well as the specialization between these vertices and $(0,0)$.

LeMmA 2.7. Let $i, j$ be nonnegative integers. The transition operators $\mathcal{U}_{i, j}^{\llcorner}$ and $\mathcal{U}_{i, i}^{\perp}$ map a half-space Macdonald process to another half-space Macdonald process as specified above if and only if they satisfy the following two equations: For all partitions $\kappa, \mu, v, \pi$ and all specializations $\rho_{\circ}, \rho_{i+1}, \rho_{j+1}$ for which normalizations remain finite,

$$
\begin{aligned}
\sum_{\mu} Q_{\kappa / \mu}\left(\rho_{i+1}\right) P_{v / \mu}\left(\rho_{j+1}\right) \mathcal{U}^{\llcorner}(\pi \mid \kappa, \mu, v) & =\frac{P_{\pi / \kappa}\left(\rho_{j+1}\right) Q_{\pi / v}\left(\rho_{i+1}\right)}{\Pi\left(\rho_{j+1}, \rho_{i+1}\right)} \\
\sum_{\mu} \frac{Q_{\kappa / \mu}\left(\rho_{i+1}\right) \mathcal{E}_{\mu}\left(\rho_{\circ}\right)}{\Pi\left(\bigcup_{e \in E^{\uparrow}(\omega)} \rho_{e} ; \rho_{i+1}\right)} \mathcal{U}^{L}(\pi \mid \kappa, \mu) & =\frac{P_{\pi / \kappa}\left(\rho_{i+1}\right) \mathcal{E}_{\pi}\left(\rho_{\circ}\right)}{\Phi\left(\bigcup_{e \in E^{\uparrow}} \rho_{e}\right)^{-1} \Phi\left(\bigcup_{e \in E^{\uparrow}} \rho_{e}, \rho_{i+1}\right)}
\end{aligned}
$$

Proof. Let us explain how these equations are derived. It will be clear from the proof why they are sufficient to define bona fide bulk and boundary operators preserving the half-space Macdonald process. We focus only on $\mathcal{U}^{L}$. The analogous result for $\mathcal{U}^{\llcorner}$can be proved similarly and was essentially already obtained in the literature (see [MP17, Eq. (2.24)] and references therein, in the context of dynamics preserving the full-space Macdonald process).

Let $\omega \in \Omega$ such that $(i, i) \in \omega$ and the edge $(i+1, i) \rightarrow(i, i) \in E(\omega)$ and let $\omega^{\prime}$ be the path obtained from $\omega$ by changing the leftward edge into $(i, i)$ to an upward edge into $(i+1, i+1)$. The fact that $\mathcal{U}_{i, i}^{L}$ maps a halfspace Macdonald process on $\omega$ to a half-space Macdonald process on $\omega^{\prime}$ with specializations chosen according to Figure 8 is equivalent to

$$
\sum_{\lambda} \mathbb{P}_{\omega, \rho}^{q, t}(\lambda) \mathcal{U}_{i, i}^{L}\left(\lambda^{(i+1, i+1)} \mid \lambda^{(i+1, i)}, \lambda^{(i, i)}\right)=\mathbb{P}_{\omega^{\prime}, \rho^{\prime}}^{q, t}\left(\lambda^{\prime}\right),
$$

where $\lambda$ and $\lambda^{\prime}$ (respectively, $\rho$ and $\rho^{\prime}$ ) are the sequences of partitions (respectively, specializations) along $\omega$ and $\omega^{\prime}$. Removing on both sides of (38) the weights corresponding to the edges shared by $\omega$ and $\omega^{\prime}$, one is left with

$$
\begin{aligned}
& \sum_{\lambda^{(i, i)}} \frac{Q_{\lambda^{(i+1, i)} / \lambda^{(i, i)}}\left(\rho_{i+1}\right) \mathcal{E}_{\lambda^{(i, i)}}\left(\rho_{\circ}\right)}{\Pi\left(\bigcup_{e \in E^{\uparrow}(\omega)} \rho_{e} ; \rho_{i+1}\right)} \mathcal{U}_{i, i}^{L}\left(\lambda^{(i+1, i+1)} \mid \lambda^{(i+1, i)}, \lambda^{(i, i)}\right) \\
& \quad=\frac{P_{\lambda^{(i+1, i+1)} / \lambda^{(i+1, i)}}\left(\rho_{i+1}\right) \mathcal{E}_{\lambda^{(i+1, i+1)}}\left(\rho_{\circ}\right)}{\Phi\left(\bigcup_{e \in E^{\uparrow}} \rho_{e}\right)^{-1} \Phi\left(\bigcup_{e \in E^{\uparrow}} \rho_{e}, \rho_{i+1}\right)},
\end{aligned}
$$

which must hold for any partitions $\lambda^{(i+1, i)}$ and $\lambda^{(i+1, i+1)}$. 
2.4.2. Push-block dynamics. It is nontrivial to solve equations (36) and (37) in general. It simplifies things considerably if we assume that $\mathcal{U}^{\llcorner}(\pi \mid \kappa, \mu, \nu)=$ $\mathcal{U}^{\llcorner}(\pi \mid \kappa, \nu)$ and $\mathcal{U}^{L}(\pi \mid \kappa, \mu)=\mathcal{U}^{\perp}(\pi \mid \kappa)$ (that is, they do not depend on the partition that is being summed over in (36) and (37)). In such a case, $\mathcal{U}^{\llcorner}(\pi \mid \kappa$, $\nu)$ and $\mathcal{U}^{-}(\pi \mid \kappa)$ factor out of the left-hand side of these equations, and we can compute the summation over $\mu$ as

$$
\begin{aligned}
\sum_{\mu} Q_{\kappa / \mu}\left(\rho_{i+1}\right) P_{v / \mu}\left(\rho_{j+1}\right) & =\sum_{\pi} \frac{P_{\pi / \kappa}\left(\rho_{j+1}\right) Q_{\pi / v}\left(\rho_{i+1}\right)}{\Pi\left(\rho_{j+1}, \rho_{i+1}\right)} \\
\sum_{\mu} \frac{Q_{\kappa / \mu}\left(\rho_{i+1}\right) \mathcal{E}_{\mu}\left(\rho_{\circ}\right)}{\Pi\left(\bigcup_{e \in E^{\uparrow}(\omega)} \rho_{e} ; \rho_{i+1}\right)} & =\frac{\mathcal{E}_{\kappa}\left(\rho_{\circ}, \rho_{i+1}\right)}{\Pi\left(\bigcup_{e \in E^{\uparrow}(\omega)} \rho_{e} ; \rho_{i+1}\right)}
\end{aligned}
$$

Thus, we may choose $\mathcal{U}_{i, j}^{\llcorner}(\pi \mid \kappa, \mu, \nu)$ as

$$
\mathcal{U}_{i, j}^{\llcorner}(\pi \mid \kappa, \mu, \nu)=\mathcal{U}_{i, j}^{\llcorner}(\pi \mid \kappa, v)=\frac{P_{\pi / \kappa}\left(\rho_{j+1}\right) Q_{\pi / v}\left(\rho_{i+1}\right)}{\sum_{\pi} P_{\pi / \kappa}\left(\rho_{j+1}\right) Q_{\pi / v}\left(\rho_{i+1}\right)} .
$$

This transition operator was introduced in [BC14, Section 2.3] following an approach introduced in the work of Diaconis-Fill [DF90] for general Markov chains and developed by Borodin-Ferrari [BF14b] in the Schur process case. The term push-block dynamics comes from [BF14b], where the dynamics on Gelfand-Tsetlin patterns (that give a way of encoding a sequence of interlacing partitions), interpreted as particle system dynamics, are described using nearest neighbor interaction of particles with pushing and blocking mechanisms. At the Macdonald process level of generality, these push-block dynamics do not admit a particularly nice interpretation in terms of local moves on a Gelfand-Tsetlin pattern. In the special case of $t=0$ (Section 4), we will recall two other choices of dynamics from [MP17] that have nicer marginals.

Using (40), the operator $\mathcal{U}_{i, i}^{\perp}$ may be chosen as

$$
\mathcal{U}_{i, i}^{L}(\pi \mid \kappa, \mu)=\mathcal{U}_{i, i}^{L}(\pi \mid \kappa)=\frac{P_{\pi / \kappa}\left(\rho_{i+1}\right) \mathcal{E}_{\pi}\left(\rho_{\circ}\right)}{\mathcal{E}_{\kappa}\left(\rho_{i+1}, \rho_{\circ}\right) \Pi\left(\rho_{i+1}, \rho_{\circ}\right) \Phi\left(\rho_{i+1}\right)} .
$$

REMARK 2.8. The above construction also provides a way to grow usual Macdonald processes [BC14]. In that case, we would consider a path starting from the horizontal axis and ending on the vertical axis, the growth mechanism would involve only the bulk transition operator $\mathcal{U}^{\llcorner}$and the set of specializations $\left\{\rho_{i}^{\mathrm{h}}\right\}_{i \geqslant 1}$ on horizontal edges and $\left\{\rho_{i}^{\mathrm{v}}\right\}_{i \geqslant 1}$ on vertical edges can be different.

REMARK 2.9. The degeneration of these dynamics when $q=t$ preserve the Pfaffian Schur process. They are studied in [BBCS18b] to justify that the first 
coordinates $\lambda_{1}$ of random partitions in a Pfaffian Schur process have the same law as last-passage times along a down-right path in a model of last-passage percolation in a half-quadrant.

\section{Observables of half-space Macdonald processes}

Consider positive variables $x_{1}, \ldots, x_{n}$ and a Macdonald nonnegative specialization $\rho$. The generalized Littlewood summation identity (30)

$$
\sum_{\lambda \in \mathbb{Y}} P_{\lambda}\left(x_{1}, \ldots, x_{n}\right) \mathcal{E}_{\lambda}(\rho)=\Pi\left(x_{1}, \ldots x_{n} ; \rho\right) \Phi\left(x_{1}, \ldots, x_{n}\right)
$$

holds as a numeric identity under some assumptions on $x_{1}, \ldots, x_{n}$ and $\rho$. Let us assume that we have a linear operator $\mathbf{A}_{n}$ which acts on $n$-variable symmetric functions and which is diagonal in the basis $\left\{P_{\lambda}\right\}$ of symmetric polynomials, with eigenvalues $\left\{d_{\lambda}\right\}$. Then, applying $\mathbf{A}_{n}$ to both sides of the above equality in the variables $x_{1}, \ldots, x_{n}$ and subsequently dividing both sides by $\Pi\left(x_{1}, \ldots x_{n} ; \rho\right)$ $\Phi\left(x_{1}, \ldots, x_{n}\right)$, we find that

$$
\begin{aligned}
\mathbb{E}_{\left(x_{1}, \ldots, x_{n}\right), \rho}^{q, t}\left[d_{\lambda}\right] & =\sum_{\lambda \in \mathbb{Y}} d_{\lambda} \frac{P_{\lambda}\left(x_{1}, \ldots, x_{n}\right) \mathcal{E}_{\lambda}(\rho)}{\Pi\left(x_{1}, \ldots x_{n} ; \rho\right) \Phi\left(x_{1}, \ldots, x_{n}\right)} \\
& =\frac{\mathbf{A}_{n} \Pi\left(x_{1}, \ldots x_{n} ; \rho\right) \Phi\left(x_{1}, \ldots, x_{n}\right)}{\Pi\left(x_{1}, \ldots x_{n} ; \rho\right) \Phi\left(x_{1}, \ldots, x_{n}\right)}
\end{aligned}
$$

where the numerator on the right-hand side $\mathbf{A}_{n} \Pi\left(x_{1}, \ldots x_{n} ; \rho\right) \Phi\left(x_{1}, \ldots, x_{n}\right)$ stands for the application of $\mathbf{A}_{n}$ to the function $\left(x_{1}, \ldots, x_{n}\right) \mapsto \Pi\left(x_{1}, \ldots x_{n} ; \rho\right)$ $\times \Phi\left(x_{1}, \ldots, x_{n}\right)$ and then subsequent evaluation at the point $\left(x_{1}, \ldots, x_{n}\right)$. If there are many operators that are all mutually diagonalized by $P_{\lambda}$, then applying them sequentially yields formulas for moments involving the products of their eigenfunctions; cf. [BC14, Section 2.2.3], where this scheme was first realized in a similar context. Note that one must check the validity of exchanging the action of $\mathbf{A}_{n}$ with the summation over $\lambda$.

\subsection{Macdonald difference operators.}

Definition 3.1. For any $u \in \mathbb{R}$ and $1 \leqslant i \leqslant n$, define the shift operator $\mathbf{T}_{u, x_{i}}$ by

$$
\left(\mathbf{T}_{u, x_{i}} F\right)\left(x_{1}, \ldots, x_{n}\right)=F\left(x_{1}, \ldots, u x_{i}, \ldots, x_{n}\right),
$$

and for any subset $I \subset\{1, \ldots, n\}$ with $r$ elements, define

$$
A_{I}(x ; t)=t^{\frac{r(r-1)}{2}} \prod_{i \in I, j \notin I} \frac{t x_{i}-x_{j}}{x_{i}-x_{j}} .
$$


For $r=1,2, \ldots, n$, define the $r$ th Macdonald difference operator

$$
\mathbf{D}_{n}^{r}=\sum_{\substack{I \subset\{1, \ldots, n\} \\|I|=r}} A_{I}(x ; t) \prod_{i \in I} \mathbf{T}_{q, x_{i}} .
$$

Also, define a variant of the Macdonald difference operator

$$
\overline{\mathbf{D}}_{n}^{r}=t^{-\frac{n(n-1)}{2}} \mathbf{D}_{n}^{n-r} \mathbf{T}_{q^{-1}},
$$

where the operator $\mathbf{T}_{q^{-1}}$ multiplies all variables by $q^{-1}, T_{q^{-1}}=T_{q^{-1}, x_{1}}, \ldots$, $T_{q^{-1}, x_{n}}$.

PROPOSITION 3.2. For any partition $\lambda$ with $\ell(\lambda) \leqslant n$,

$$
\begin{aligned}
\mathbf{D}_{n}^{r} P_{\lambda}\left(x_{1}, \ldots, x_{n}\right) & =e_{r}\left(q^{\lambda_{1}} t^{n-1}, q^{\lambda_{2}} t^{n-2}, \ldots, q^{\lambda_{n}} t^{0}\right) P_{\lambda}\left(x_{1}, \ldots, x_{n}\right), \\
\overline{\mathbf{D}}_{n}^{r} P_{\lambda}\left(x_{1}, \ldots, x_{n}\right) & =t^{-\frac{n(n-1)}{2}} q^{-|\lambda|} e_{n-r}\left(q^{\lambda_{1}} t^{n-1}, \ldots, q^{\lambda_{n}} t^{0}\right) P_{\lambda}\left(x_{1}, \ldots, x_{n}\right) \\
& =e_{r}\left(q^{-\lambda_{1}} t^{1-n}, q^{-\lambda_{2}} t^{2-n}, \ldots, q^{-\lambda_{n}} t^{0}\right) P_{\lambda}\left(x_{1}, \ldots, x_{n}\right) .
\end{aligned}
$$

Here $e_{r}$ is the elementary symmetric function,

$$
e_{r}\left(x_{1}, \ldots, x_{n}\right)=\sum_{1 \leqslant i_{1}<\cdots<i_{r} \leqslant n} x_{i_{1}} \cdots x_{i_{r}} .
$$

Proof. The first identity is from [Mac95, VI(4.15)] and the next two follow easily, as explained in [BC14, Remark 2.2.12].

PROPOSITION 3.3. Fix $k \geqslant 1$ and consider functions $H\left(u_{1}, \ldots, u_{n}\right)=$ $\prod_{i=1}^{n} h\left(u_{i}\right)$ and $\Phi\left(u_{1}, \ldots, u_{n}\right)=\prod_{i<j} \phi\left(u_{i} u_{j}\right)$, where $h(\cdot)$ and $\phi(\cdot)$ are univariate locally holomorphic functions as specified below. Then

$$
\begin{aligned}
& \frac{\left(\left(\mathbf{D}_{n}^{1}\right)^{k}(H \Phi)\right)\left(x_{1}, \ldots, x_{n}\right)}{(H \Phi)\left(x_{1}, \ldots, x_{n}\right)}=\frac{(t-1)^{-k}}{(2 \pi \mathbf{i})^{k}} \oint \ldots \oint \\
& \quad \times \prod_{1 \leqslant a<b \leqslant k} \frac{\left(t w_{a}-q w_{b}\right)\left(w_{a}-w_{b}\right)}{\left(w_{a}-q w_{b}\right)\left(t w_{a}-w_{b}\right)} \frac{\phi\left(q^{2} w_{a} w_{b}\right) \phi\left(w_{a} w_{b}\right)}{\phi\left(q w_{a} w_{b}\right)^{2}} \\
& \quad \times \prod_{m=1}^{k} \prod_{j=1}^{n}\left(\frac{t w_{m}-x_{j}}{w_{m}-x_{j}} \frac{\phi\left(q x_{j} w_{m}\right)}{\phi\left(x_{j} w_{m}\right)}\right) \frac{h\left(q w_{m}\right) \phi\left(w_{m}^{2}\right)}{h\left(w_{m}\right) \phi\left(q w_{m}^{2}\right)} \frac{d w_{m}}{w_{m}}
\end{aligned}
$$

and

$$
\frac{\left(\left(t^{n-1} \overline{\mathbf{D}}_{n}^{1}\right)^{k}(H \Phi)\right)\left(x_{1}, \ldots, x_{n}\right)}{(H \Phi)\left(x_{1}, \ldots, x_{n}\right)}=\frac{(t-1)^{-k}}{(2 \pi \mathbf{i})^{k}} \oint \cdots \oint
$$




$$
\begin{aligned}
& \times \prod_{1 \leqslant a<b \leqslant k} \frac{\left(t w_{a}-q w_{b}\right)\left(w_{a}-w_{b}\right)}{\left(w_{a}-q w_{b}\right)\left(t w_{a}-w_{b}\right)} \frac{\phi\left(\frac{1}{q^{2} w_{a} w_{b}}\right) \phi\left(\frac{1}{w_{a} w_{b}}\right)}{\phi\left(\frac{1}{q w_{a} w_{b}}\right)^{2}} \\
& \times \prod_{m=1}^{k} \prod_{j=1}^{n}\left(\frac{1-t w_{m} x_{j}}{1-w_{m} x_{j}} \frac{\phi\left(\frac{x_{j}}{q w_{m}}\right)}{\phi\left(\frac{x_{j}}{w_{m}}\right)}\right) \frac{h\left(\frac{1}{q w_{m}}\right) \phi\left(\frac{1}{w_{m}^{2}}\right)}{h\left(\frac{1}{w_{m}}\right) \phi\left(\frac{1}{q w_{m}^{2}}\right)} \frac{d w_{m}}{w_{m}} .
\end{aligned}
$$

There are some assumptions we must make on the contours and functions $h$, $\phi$ for the above equalities to hold. In the first formula, we assume that the (positively oriented) contour for $w_{c}, 1 \leqslant c \leqslant k$, contains $\left\{x_{1}, \ldots, x_{n}\right\}$ and the image of the $w_{c+1}, \ldots, w_{k}$ contours multiplied by $q$, and does not contain any other singularities of the integrand. In the second formula, we assume that the (positively oriented) contour for $w_{c}, 1 \leqslant c \leqslant k$, contains $\left\{x_{1}^{-1}, \ldots, x_{n}^{-1}\right\}$ and the image of the $w_{c+1}, \ldots, w_{k}$ contours multiplied by $q$, and does not contain any other singularities of the integrand. In both cases, we also assume that $h$ and $\phi$ are holomorphic and nonzero on a suitably large complex neighborhood so as not to yield any singularities when the integrals are evaluated through residues by shrinking the contours.

Proof. We prove the first equality (the second follows similarly) via the approach used in the proofs of [BC14, Propositions 2.2.11, 2.2.14] (which correspond to the special case when $\phi$ is assumed to be constant). Let us consider the effect of the $\phi$ terms. Observe that due to the multiplicative structure of $\Phi$,

$$
\frac{\left(\mathbf{T}_{q, x_{j}} \Phi\right)\left(x_{1}, \ldots, x_{n}\right)}{\Phi\left(x_{1}, \ldots, x_{n}\right)}=\operatorname{Subs}_{z=x_{j}} \frac{\phi\left(z^{2}\right)}{\phi\left(q z^{2}\right)} \prod_{i=1}^{n} \frac{\phi\left(q x_{i} z\right)}{\phi\left(x_{i} z\right)},
$$

where $\operatorname{Subs}_{z=x}$ means the substitution of $z=x$ into the expression that follows. Combining with the proof of [BC14, Propositions 2.2.11], we arrive at

$$
\begin{aligned}
\left(\mathbf{D}_{n}^{1}(H \Phi)\right)\left(x_{1}, \ldots, x_{n}\right)= & \frac{(t-1)^{-1}}{2 \pi \mathbf{i}} \oint \prod_{j=1}^{n}\left(\frac{t w_{1}-x_{j}}{w_{1}-x_{j}} \frac{\phi\left(q x_{j} w_{1}\right)}{\phi\left(x_{j} w_{1}\right)}\right) \\
& \times \frac{h\left(q w_{1}\right) \phi\left(w_{1}^{2}\right)}{h\left(w_{1}\right) \phi\left(q w_{1}^{2}\right)} \frac{d w_{1}}{w_{1}}(H \Phi)\left(x_{1}, \ldots, x_{n}\right),
\end{aligned}
$$

which is the $k=1$ case of the proposition we seek to prove.

Observe that inside the integral formula for $\left(\mathbf{D}_{n}^{1}(H \Phi)\right)\left(x_{1}, \ldots, x_{n}\right)$, the variables $x_{1}, \ldots, x_{n}$ arise in the terms

$$
(H \Phi)\left(x_{1}, \ldots, x_{n}\right) \prod_{j=1}^{n} \frac{t w_{1}-x_{j}}{w_{1}-x_{j}} \frac{\phi\left(q x_{j} w_{1}\right)}{\phi\left(x_{j} w_{1}\right)},
$$


which is equal to $H_{1} \Phi$ where $H_{1}\left(x_{1}, \ldots, x_{n}\right):=\prod_{i=1}^{N} h_{1}\left(x_{i}\right)$ with

$$
h_{1}(x):=h(x) \frac{t w_{1}-x}{w_{1}-x} \frac{\phi\left(q x w_{1}\right)}{\phi\left(x w_{1}\right)} .
$$

Applying $\mathbf{D}_{n}^{1}$, we find that by linearity it can be taken into the integrand and it applies just to $H_{1} \Phi$, which introduces a second integral (appealing to the $k=1$ case we have already proved). We obtain

$$
\begin{aligned}
\left(\left(\mathbf{D}_{n}^{1}\right)^{2}(H \Phi)\right)\left(x_{1}, \ldots, x_{n}\right) & \\
= & \frac{(t-1)^{-2}}{(2 \pi \mathbf{i})^{2}} \oint \oint \frac{\left(t w_{1}-q w_{2}\right)\left(w_{1}-w_{2}\right)}{\left(w_{1}-q w_{2}\right)\left(t w_{1}-w_{2}\right)} \\
& \times \frac{\phi\left(q^{2} w_{1} w_{2}\right) \phi\left(w_{1} w_{2}\right)}{\phi\left(q w_{1} w_{2}\right)^{2}} \prod_{j=1}^{n}\left(\frac{t w_{2}-x_{j}}{w_{2}-x_{j}} \frac{\phi\left(q x_{j} w_{2}\right)}{\phi\left(x_{j} w_{2}\right)}\right) \frac{h\left(q w_{2}\right) \phi\left(w_{2}^{2}\right)}{h\left(w_{2}\right) \phi\left(q w_{2}^{2}\right)} \frac{d w_{2}}{w_{2}} \\
& \times \prod_{j=1}^{n}\left(\frac{t w_{1}-x_{j}}{w_{1}-x_{j}} \frac{\phi\left(q x_{j} w_{1}\right)}{\phi\left(x_{j} w_{1}\right)}\right) \frac{h\left(q w_{1}\right) \phi\left(w_{1}^{2}\right)}{h\left(w_{1}\right) \phi\left(q w_{1}^{2}\right)} \frac{d w_{1}}{w_{1}}(H \Phi)\left(x_{1}, \ldots, x_{n}\right),
\end{aligned}
$$

where the contour for $w_{1}$ encircles the $x_{i}$ 's and $q w_{2}$, and the contour for $w_{2}$ encircles the $x_{i}$ 's. Now the variables $x_{1}, \ldots, x_{n}$ arise inside the double integral as $H_{2} \Phi$, where $H_{2}\left(x_{1}, \ldots, x_{n}\right)=\prod_{i=1}^{n} h_{2}\left(x_{i}\right)$ with

$$
h_{2}(x)=h(x) \frac{t w_{2}-x}{w_{2}-x} \frac{t w_{1}-x}{w_{1}-x} \frac{\phi\left(q x w_{1}\right)}{\phi\left(x w_{1}\right)} \frac{\phi\left(q x w_{2}\right)}{\phi\left(x w_{2}\right)} .
$$

Repeating $k$ times leads to the claimed formula.

Proposition 3.4. Fix $r \geqslant 1, H\left(u_{1}, \ldots, u_{n}\right)=\prod_{i=1}^{n} h\left(u_{i}\right)$ and $\Phi\left(u_{1}, \ldots\right.$, $\left.u_{n}\right)=\prod_{i<j} \phi\left(u_{i} u_{j}\right)$, where $h$ and $\phi$ are holomorphic and nonzero on a suitably large complex neighborhood so as not to yield any singularities when both integrals below are evaluated through residues. Then

$$
\begin{aligned}
& \frac{\mathbf{D}_{n}^{r}(H \Phi)\left(x_{1}, \ldots, x_{n}\right)}{(H \Phi)\left(x_{1}, \ldots, x_{n}\right)} \\
& =\frac{1}{r !} \oint \frac{d z_{1}}{2 \mathbf{i} \pi} \cdots \oint \frac{d z_{r}}{2 \mathbf{i} \pi} \operatorname{det}\left[\frac{1}{t z_{k}-z_{l}}\right]_{k, l=1}^{r} \\
& \quad \times \prod_{j=1}^{r}\left(\frac{h\left(q z_{j}\right)}{h\left(z_{j}\right)} \prod_{m=1}^{n} \frac{t z_{j}-x_{m}}{z_{j}-x_{m}}\right) \prod_{1 \leqslant i<j \leqslant r} \frac{\phi\left(q^{2} z_{i} z_{j}\right)}{\phi\left(z_{i} z_{j}\right)} \\
& \quad \times \prod_{i=1}^{n} \prod_{j=1}^{r} \frac{\phi\left(q z_{j} x_{i}\right)}{\phi\left(z_{j} x_{i}\right)} \prod_{i, j=1}^{r} \frac{\phi\left(z_{i} z_{j}\right)}{\phi\left(q z_{i} z_{j}\right)},
\end{aligned}
$$


where the contours are positively oriented contours encircling $\left\{x_{1}, \ldots, x_{n}\right\}$ and no other singularity of the integrand.

We also have

$$
\begin{aligned}
& \frac{\overline{\mathbf{D}}_{n}^{r}(H \Phi)\left(x_{1}, \ldots, x_{n}\right)}{(H \Phi)\left(x_{1}, \ldots, x_{n}\right)} \\
& =\frac{1}{r !} \oint \frac{d w_{1}}{2 \mathbf{i} \pi} \cdots \oint \frac{d w_{r}}{2 \mathbf{i} \pi} \operatorname{det}\left[\frac{1}{t w_{k}-w_{l}}\right]_{k, l=1}^{r} \\
& \quad \times \prod_{j=1}^{r}\left(\frac{h\left(\left(q w_{j}\right)^{-1}\right)}{h\left(w_{j}^{-1}\right)} \prod_{m=1}^{n} \frac{t w_{j}-x_{m}^{-1}}{w_{j}-x_{m}^{-1}}\right) \prod_{1 \leqslant i<j \leqslant r} \frac{\phi\left(\left(q^{2} w_{i} w_{j}\right)^{-1}\right)}{\phi\left(\left(w_{i} w_{j}\right)^{-1}\right)} \\
& \quad \times \prod_{i=1}^{n} \prod_{j=1}^{r} \frac{\phi\left(x_{i} /\left(q w_{j}\right)\right)}{\phi\left(x_{i} / w_{j}\right)} \prod_{i, j=1}^{r} \frac{\phi\left(\left(w_{i} w_{j}\right)^{-1}\right)}{\phi\left(\left(q w_{i} w_{j}\right)^{-1}\right)},
\end{aligned}
$$

where the contours are positively oriented circles encircling $\left\{1 / x_{1}, \ldots, 1 / x_{n}\right\}$ and no other singularity.

Proof. Note that for $|I|=r$, one has that

$$
\begin{aligned}
\prod_{i \in I} & \frac{\mathbf{T}_{q, x_{i}} \Phi}{\Phi}\left(x_{1}, \ldots, x_{n}\right) \\
= & \operatorname{Subs}_{\substack{z_{1}=x_{i_{1}} \\
z_{r}=x_{i r}}}\left[\prod_{1 \leqslant<i<j \leqslant r} \frac{\phi\left(q^{2} z_{i} z_{j}\right)}{\phi\left(z_{i} z_{j}\right)} \prod_{i=1}^{n} \prod_{j=1}^{r} \frac{\phi\left(q z_{j} x_{i}\right)}{\phi\left(z_{j} x_{i}\right)} \prod_{i, j=1}^{r} \frac{\phi\left(z_{i} z_{j}\right)}{\phi\left(q z_{i} z_{j}\right)}\right] .
\end{aligned}
$$

Then, one proceeds as in [BC14, Proposition 2.2.11] evaluating the integral through the residues at $x_{1}, \ldots, x_{n}$.

3.2. Moment integral formulas. In this section, we consider the half-space Macdonald measure with specializations $\rho=\left(a_{1}, \ldots, a_{n}\right)$, where $a_{i} \in(0,1)$, and $\rho^{\swarrow}=\rho$, where $\rho$ is a Macdonald nonnegative specialization such that $\Pi(\vec{a} ; \rho) \Phi(\vec{a})$ can be expanded via the generalized Littlewood identity (30).

PROPOSITION 3.5. For any positive integer $k$,

$$
\begin{aligned}
& \mathbb{E}_{\left(a_{1}, \ldots, a_{n}\right), \rho}^{q, t}\left[\left(q^{\lambda_{1}} t^{n-1}+q^{\lambda_{2}} t^{n-2}+\cdots+q^{\lambda_{n}}\right)^{k}\right] \\
& \quad=\frac{1}{(t-1)^{k}} \oint \frac{d w_{1}}{2 \mathbf{i} \pi} \cdots \oint \frac{d w_{k}}{2 \mathbf{i} \pi}
\end{aligned}
$$




$$
\begin{aligned}
& \times \prod_{1 \leqslant a<b \leqslant k} \frac{\left(t w_{a}-q w_{b}\right)\left(w_{a}-w_{b}\right)}{\left(w_{a}-q w_{b}\right)\left(t w_{a}-w_{b}\right)} \frac{\left(1-q w_{a} w_{b}\right)\left(1-t w_{a} w_{b}\right)}{\left(1-q t w_{a} w_{b}\right)\left(1-w_{a} w_{b}\right)} \\
& \times \prod_{m=1}^{k}\left(\prod_{j=1}^{n} \frac{t w_{m}-a_{j}}{w_{m}-a_{j}} \frac{1-a_{j} w_{m}}{1-t a_{j} w_{m}}\right) \frac{\Pi\left(q w_{m} ; \rho\right)\left(1-t w_{m}^{2}\right)}{\Pi\left(w_{m} ; \rho\right)\left(1-w_{m}^{2}\right)} \frac{1}{w_{m}}
\end{aligned}
$$

where the contour for $w_{c}, 1 \leqslant c \leqslant k$, contains $\left\{a_{1}, \ldots, a_{n}\right\}$ and the image of the $w_{c+1}, \ldots, w_{k}$ contours multiplied by $q$, and does not contain any other singularities of the integrand (this may restrict the choice of admissible specializations $\rho$ ).

Similarly, assume that $\rho$ and $k$ are chosen so that $\Pi\left(q^{-k} \vec{a} ; \rho\right) \Phi\left(q^{-k} \vec{a}\right)$ can be expanded via the generalized Littlewood identity (30). Then we also have

$$
\begin{aligned}
& \mathbb{E}_{\left(a_{1}, \ldots, a_{n}\right), \rho}^{q, t}\left[\left(q^{-\lambda_{1}}+q^{-\lambda_{2}} t+\cdots+q^{-\lambda_{n}} t^{n-1}\right)^{k}\right]=\frac{1}{(t-1)^{k}} \oint \frac{d w_{1}}{2 \mathbf{i} \pi} \cdots \oint \frac{d w_{k}}{2 \mathbf{i} \pi} \\
& \times \prod_{1 \leqslant a<b \leqslant k} \frac{\left(t w_{a}-q w_{b}\right)\left(w_{a}-w_{b}\right)}{\left(w_{a}-q w_{b}\right)\left(t w_{a}-w_{b}\right)} \frac{\left(q^{2} w_{a} w_{b}-t\right)\left(q w_{a} w_{b}-1\right)}{\left(q^{2} w_{a} w_{b}-1\right)\left(q w_{a} w_{b}-t\right)} \\
& \quad \times \prod_{m=1}^{k}\left(\prod_{j=1}^{n} \frac{1-t w_{m} a_{j}}{1-w_{m} a_{j}} \frac{q w_{m}-t a_{j}}{q w_{m}-a_{j}}\right) \frac{\Pi\left(\left(q w_{m}\right)^{-1} ; \rho\right)\left(q w_{m}^{2}-1\right)}{\Pi\left(\left(w_{m}\right)^{-1} ; \rho\right)\left(q w_{m}^{2}-t\right)} \frac{1}{w_{m}},
\end{aligned}
$$

where the contour for $w_{c}, 1 \leqslant c \leqslant k$, contains $\left\{1 / a_{1}, \ldots, 1 / a_{n}\right\}$ and the image of the $w_{c+1}, \ldots, w_{k}$ contours multiplied by $q$, and does not contain any other singularities of the integrand (this may restrict the choice of admissible specializations $\rho$ ). All contours above are positively oriented.

Proof. Plug in $h(u)=\Pi(u ; \rho)$ and $\phi(u)=\frac{(t u ; q)_{\infty}}{(u ; q)_{\infty}}$ in Proposition 3.3. This yields integral formulas for $\frac{\left(\mathbf{D}_{n}^{1}\right)^{k} \Pi(\vec{a}, \rho) \Phi(\vec{a})}{\Pi(\vec{a}, \rho) \Phi(\vec{a})}$ and $\frac{\left(\overline{\mathbf{D}}_{n}^{1}\right)^{k} \Pi(\vec{a}, \rho) \Phi(\vec{a})}{\Pi(\vec{a}, \rho) \Phi(\vec{a})}$, where the operators act on the variables $a_{i}$. These quantities are related to the desired expectations by Proposition 3.2. In (47), we need the extra assumptions on $\rho$ that $\Pi\left(q^{-k} \vec{a} ; \rho\right) \Phi\left(q^{-k} \vec{a}\right)$ can be expanded via the generalized Littlewood identity (30) to ensure that one can commute the action of the operator $\left(\overline{\mathbf{D}}_{n}^{1}\right)^{k}$ with the expectation $\mathbb{E}_{\left(a_{1}, \ldots, a_{n}\right), \rho}^{q, t}$ and one obtains using the Fubini theorem that

$$
\frac{\left(\overline{\mathbf{D}}_{n}^{1}\right)^{k} \Pi(\vec{a}, \rho) \Phi(\vec{a})}{\Pi(\vec{a}, \rho) \Phi(\vec{a})}=\mathbb{E}_{\left(a_{1}, \ldots, a_{n}\right), \rho}^{q, t}\left[\left(q^{-\lambda_{1}}+q^{-\lambda_{2}} t+\cdots+q^{-\lambda_{n}} t^{n-1}\right)^{k}\right]
$$

REMARK 3.6. If $\rho$ is of the form $\rho=\rho(\alpha, \beta, \gamma)$ as in Section 2.2.4, the hypothesis that $\left.\Pi\left(q^{-k} \vec{a}\right) ; \rho\right) \Phi\left(q^{-k} \vec{a}\right)$ can be expanded via the generalized 
Littlewood identity (30) is equivalent to the fact that $\max _{i}\left\{\alpha_{i}\right\} \max _{i}\left\{a_{i}\right\}<q^{k}$ and $\left(\max _{i}\left\{a_{i}\right\}\right)^{2}<q^{k}$. Otherwise, the expectation on the left-hand side in (47) would fail to exist (see Section 2.2.4).

PROPOSITION 3.7. Let $r$ be a positive integer. We have

$$
\begin{aligned}
\mathbb{E}_{\left(a_{1}, \ldots, a_{n}\right), \rho}^{q, t}\left[e_{r}\left(q^{\lambda_{1}} t^{n-1}, q^{\lambda_{2}} t^{n-2}, \ldots, q^{\lambda_{n}}\right)\right] \\
=\frac{1}{r !} \oint \frac{d z_{1}}{2 \mathbf{i} \pi} \cdots \oint \frac{d z_{r}}{2 \mathbf{i} \pi} \\
\quad \times \operatorname{det}\left[\frac{1}{t z_{k}-z_{l}}\right]_{k, l=1}^{r} \prod_{j=1}^{r}\left(\frac{\Pi\left(q z_{j} ; \rho\right)}{\Pi\left(z_{j} ; \rho\right)} \prod_{i=1}^{n}\left(\frac{t z_{j}-a_{i}}{z_{j}-a_{i}} \frac{1-z_{j} a_{i}}{1-t z_{j} a_{i}}\right)\right) \\
\quad \times \prod_{i=1}^{r} \frac{1-t z_{i}^{2}}{1-z_{i}^{2}} \prod_{1 \leqslant i<j \leqslant r} \frac{1-q z_{i} z_{j}}{1-t q z_{i} z_{j}} \frac{1-t z_{i} z_{j}}{1-z_{i} z_{j}}
\end{aligned}
$$

where the contours are positively oriented closed curves around $\left\{a_{1}, \ldots, a_{n}\right\}$ and no other singularity of the integrand.

Moreover, assume that $\rho$ and $r$ are chosen so that $\Pi\left(q^{-r} \vec{a} ; \rho\right) \Phi\left(q^{-r} \vec{a}\right)$ can be expanded via the generalized Littlewood identity (30). Then,

$$
\begin{aligned}
& \mathbb{E}_{\left(a_{1}, \ldots, a_{n}\right), \rho}^{q, t}\left[e_{r}\left(q^{-\lambda_{1}} t^{1-n}, q^{-\lambda_{2}} t^{2-n}, \ldots, q^{-\lambda_{n}}\right)\right]=\frac{1}{r !} \oint \frac{d w_{1}}{2 \mathbf{i} \pi} \cdots \oint \frac{d w_{r}}{2 \mathbf{i} \pi} \\
& \quad \times \operatorname{det}\left[\frac{1}{t w_{k}-w_{l}}\right]_{k, l=1}^{r} \prod_{j=1}^{r}\left(\frac{\Pi\left(\left(q w_{j}\right)^{-1} ; \rho\right)}{\Pi\left(w_{j}^{-1} ; \rho\right)} \prod_{i=1}^{n}\left(\frac{1-a_{i} t w_{j}}{1-a_{i} w_{j}} \frac{q w_{j}-t a_{i}}{q w_{j}-a_{i}}\right)\right) \\
& \quad \times \prod_{i=1}^{r} \frac{q w_{i}^{2}-1}{q w_{i}^{2}-t} \prod_{1 \leqslant i<j \leqslant r} \frac{\left(q^{2} w_{i} w_{j}-t\right)\left(q w_{i} w_{j}-1\right)}{\left(q^{2} w_{i} w_{j}-1\right)\left(q w_{i} w_{j}-t\right)}
\end{aligned}
$$

where the contours are positively oriented closed curves around $\left\{1 / a_{1}, \ldots\right.$, $\left.1 / a_{n}\right\}$ and no other singularity.

Proof. The proof is similar as the proof of Proposition 3.5, using Proposition 3.4 instead of Proposition 3.3.

3.3. Noumi's $q$-integral operator and variants. In order to lighten some notations, we set in this section $(a)_{n}=(a ; q)_{n}$ for any $n \in \mathbb{Z}_{\geqslant 0} \cup\{\infty\}$, and we will use the function $\phi(x)=(t x)_{\infty} /(x)_{\infty}$ from (22) and the function $f(x)=$ $(t x)_{\infty} /(q x)_{\infty}$ from (18). 
Define the operator $\mathbf{N}_{n}^{z}$ acting on analytic functions in $x_{1}, \ldots, x_{n}$ by

$$
\mathbf{N}_{n}^{z}=\sum_{\eta_{1}, \ldots, \eta_{n}=0}^{\infty} z^{|\eta|} h_{\eta}(x ; q, t) \prod_{i=1}^{n}\left(\mathbf{T}_{q, x_{i}}\right)^{\eta_{i}}
$$

where $|\eta|=\eta_{1}+\cdots+\eta_{n}$ and

$$
\begin{aligned}
h_{\eta}(x ; q, t)= & \prod_{i=1}^{n} t^{(i-1) \eta_{i}} \frac{(t)_{\infty}\left(q^{\eta_{i}+1}\right)_{\infty}}{\left(t q^{\eta_{i}}\right)_{\infty}(q)_{\infty}} \\
& \times \prod_{i<j} \frac{\left(q^{\eta_{i}-\eta_{j}} x_{i} / x_{j}\right)_{\infty}\left(q^{1-\eta_{j}} t^{-1} x_{i} / x_{j}\right)_{\infty}\left(t x_{i} / x_{j}\right)_{\infty}\left(q^{1+\eta_{i}} x_{i} / x_{j}\right)_{\infty} x_{\infty}\left(q t^{-1} x_{i} / x_{j}\right)_{\infty}\left(t q^{\eta_{i}} x_{i} / x_{j}\right)_{\infty}\left(q^{1+\eta_{i}-\eta_{j}} x_{i} / x_{j}\right)_{\infty}}{\left(q^{-\eta}\right.} .
\end{aligned}
$$

Proposition 2.2.17 in [BC14] states that for $|z|<1$,

$$
\mathbf{N}_{n}^{z} P_{\lambda}(x)=\prod_{i=1}^{n} \frac{\left(q^{\lambda_{i}} t^{n-i+1} z\right)_{\infty}}{\left(q^{\lambda_{i}} t^{n-i} z\right)_{\infty}} P_{\lambda}(x)
$$

(More precisely, [BC14, Proposition 2.2.17] states the identity as a formal power series in $z$, and one can see that the right-hand side is a convergent series when $|z|<1$ using the $q$-binomial theorem (12).)

PROPOSITION 3.8. The operator $\mathbf{N}^{z}$ can be rewritten as

$$
\mathbf{N}_{n}^{z}=\sum_{\eta_{1}, \ldots, \eta_{n}=0}^{\infty} z^{|\eta|} \prod_{i<j} \frac{q^{\eta_{j}} x_{j}-q^{\eta_{i}} x_{i}}{x_{j}-x_{i}} \prod_{i, j} \frac{\left(t x_{i} / x_{j}\right)_{\eta_{i}}}{\left(q x_{i} / x_{j}\right)_{\eta_{i}}} \prod_{i=1}^{n}\left(\mathbf{T}_{q, x_{i}}\right)^{\eta_{i}} .
$$

REMARK 3.9. Proposition 3.8 shows that $\mathbf{N}_{n}^{z}$ coincides with an operator known as Noumi's q-integral operator. The eigenrelation (50) first appeared in [FHH+09], where it is attributed to [NS]. Additional properties of $\mathbf{N}_{n}^{z}$ can be found in [NS12], which in particular gives a proof of (50) (see around equation (5.6)). Note that [BCGS16, Section 5] provides yet another proof of (50) by E. Rains.

Proof. We need to show that

$$
h_{\eta}(x ; q, t)=\prod_{i<j} \frac{q^{\eta_{j}} x_{j}-q^{\eta_{i}} x_{i}}{x_{j}-x_{i}} \prod_{i, j} \frac{\left(t x_{i} / x_{j}\right)_{\eta_{i}}}{\left(q x_{i} / x_{j}\right)_{\eta_{i}}} .
$$

On the left-hand side, we have

$$
h_{\eta}(x ; q, t)=\prod_{i=1}^{n} \frac{f(1)}{f\left(q^{\eta_{i}}\right)} \prod_{i<j} \frac{t^{\eta_{j}}\left(1-q^{\eta_{i}-\eta_{j}} x_{i} / x_{j}\right)\left(t x_{i} / x_{j}\right)_{\infty}}{f\left(q^{-\eta_{j}} t^{-1} x_{i} / x_{j}\right) f\left(q^{\eta_{i}} x_{i} / x_{j}\right)\left(q t^{-1} x_{i} / x_{j}\right)_{\infty}}
$$




$$
=\prod_{i=1}^{n} \frac{f(1)}{f\left(q^{\eta_{i}}\right)} \prod_{i<j} \frac{t^{\eta_{j}}\left(1-q^{\eta_{i}-\eta_{j}} x_{i} / x_{j}\right) f\left(x_{i} / x_{j}\right) f\left(t^{-1} x_{i} / x_{j}\right)}{\left(1-x_{i} / x_{j}\right) f\left(q^{-\eta_{j}} t^{-1} x_{i} / x_{j}\right) f\left(q^{\eta_{i}} x_{i} / x_{j}\right)}
$$

On the other hand,

$$
\frac{\left(t x_{i} / x_{j}\right)_{\eta_{i}}}{\left(q x_{i} / x_{j}\right)_{\eta_{i}}}=\frac{f\left(x_{i} / x_{j}\right)}{f\left(q^{\eta_{i}} x_{i} / x_{j}\right)}
$$

so that

$$
\begin{aligned}
& \text { R.H.S. (51) } \\
& \qquad=\prod_{i=1}^{n} \frac{f(1)}{f\left(q^{\eta_{i}}\right)} \prod_{i<j} \frac{q^{\eta_{j}}\left(1-q^{\eta_{i}-\eta_{j}} x_{i} / x_{j}\right)}{1-x_{i} / x_{j}} \frac{f\left(x_{i} / x_{j}\right)}{f\left(q^{\eta_{i}} x_{i} / x_{j}\right)} \frac{f\left(x_{j} / x_{i}\right)}{f\left(q^{\eta_{j}} x_{j} / x_{i}\right)} .
\end{aligned}
$$

Thus, it is sufficient to show that

$$
\frac{t^{\eta_{j}} f\left(t^{-1} x_{i} / x_{j}\right)}{f\left(q^{-\eta_{j}} t^{-1} x_{i} / x_{j}\right)}=\frac{q^{\eta_{j}} f\left(x_{j} / x_{i}\right)}{f\left(q^{\eta_{j}} x_{j} / x_{i}\right)}
$$

which is equivalent to

$$
\frac{t^{\eta_{j}}\left(q^{1-\eta_{j}} t^{-1} x_{i} / x_{j}\right)_{\eta_{j}}}{\left(q^{-\eta_{j}} x_{i} / x_{j}\right)_{\eta_{j}}}=\frac{q^{\eta_{j}}\left(t x_{j} / x_{i}\right)_{\eta_{j}}}{\left(q x_{j} / x_{i}\right)_{\eta_{j}}}
$$

It is easy to check that

$$
\frac{t^{N}\left(q^{1-N} t^{-1} X\right)_{N}}{q^{N}\left(q^{-N} X\right)_{N}}=\frac{\left(t X^{-1}\right)_{N}}{\left(q X^{-1}\right)_{N}}
$$

so that (52) is established by setting $X=x_{i} / x_{j}, N=\eta_{j}$.

In Section 3.1, we discussed two types of Macdonald difference operators $\mathbf{D}^{r}$ and $\overline{\mathbf{D}}^{r}$. We define now an operator $\overline{\mathbf{N}}_{n}^{z}$, similar to $\mathbf{N}_{n}^{z}$, but having eigenvalue $\prod_{i=1}^{n} \frac{\left(q^{-\lambda} i t^{i} z\right)_{\infty}}{\left(q^{-\lambda_{i}} t^{i-1} z\right)_{\infty}}$ instead of $\prod_{i=1}^{n} \frac{\left(q^{\lambda_{i}} t^{n-i+1} z\right)_{\infty}}{\left(q^{\lambda_{i}} t^{n-i} z\right)_{\infty}}$. Let

$$
\overline{\mathbf{N}}_{n}^{z}=\sum_{\eta_{1}, \ldots, \eta_{n}=0}^{\infty} z^{|\eta|} h_{\eta}\left(x_{1}^{-1}, \ldots, x_{n}^{-1} ; q, t\right) \prod_{i=1}^{n}\left(\mathbf{T}_{q^{-1}, x_{i}}\right)^{\eta_{i}}
$$

Equivalently, using Proposition 3.8,

$$
\overline{\mathbf{N}}_{n}^{z}=\sum_{\eta_{1}, \ldots, \eta_{n}=0}^{\infty} z^{|\eta|} \prod_{i<j} \frac{q^{\eta_{j}} x_{i}-q^{\eta_{i}} x_{j}}{x_{i}-x_{j}} \prod_{i, j} \frac{\left(t x_{i} / x_{j}\right)_{\eta_{j}}}{\left(q x_{i} / x_{j}\right)_{\eta_{j}}} \prod_{i=1}^{n}\left(\mathbf{T}_{q^{-1}, x_{i}}\right)^{\eta_{i}}
$$


PROPOSITION 3.10 [NS12]. We have the following formal power series identity in the variable $z$ :

$$
\overline{\mathbf{N}}_{n}^{z} P_{\lambda}(x)=\prod_{i=1}^{n} \frac{\left(q^{-\lambda_{i}} t^{i} z\right)_{\infty}}{\left(q^{-\lambda_{i}} t^{i-1} z\right)_{\infty}} P_{\lambda}(x)
$$

Moreover, when $z$ is such that for all $i=1, \ldots n,\left|z q^{-\lambda_{i}} t^{i-1}\right|<1$, the identity above is an equality of absolutely convergent series.

Although this eigenrelation can be deduced from [NS12, (5.13)], we give another proof in the spirit of [BC14, Proposition 2.2.17].

Proof.

Step 1: Let us show that identity (53) holds for a certain set of specializations of $x_{i}$. For the moment, we work with formal power series in the variable $z$. Let $u_{\mu}$ be the specialization (of the ring of rational functions) that substitutes $q^{\mu_{i}} t^{n-i}$ in place of $x_{i}$ for all $i$. Let $\bar{u}_{\mu}$ be the specialization that substitutes $q^{-\mu_{i}} t^{i-n}$ in place of the variable $x_{i}$. The eigenvalue appearing in (53) can be rewritten as

$$
\prod_{i=1}^{n} \frac{\left(q^{-\lambda_{i}} t^{i} z\right)_{\infty}}{\left(q^{-\lambda_{i}} t^{i-1} z\right)_{\infty}}=\bar{u}_{\lambda}\left(\Pi\left(z t^{n-1}, x\right)\right)=\bar{u}_{\lambda}\left(\sum_{m \geqslant 0} g_{m}(x ; q, t) z^{m} t^{(n-1) m}\right),
$$

where $g_{m}$ is the $q, t$ analogue of complete homogeneous symmetric functions, that is, $g_{m}=Q_{(m)}$.

We now show that equality (53) holds under specialization $\bar{u}_{\mu}$ for any $\mu$. Macdonald symmetric polynomials satisfy an index-variable duality relation

$$
u_{\mu}\left(P_{\lambda}\right)=\frac{u_{0}\left(P_{\lambda}\right)}{u_{0}\left(P_{\mu}\right)} u_{\lambda}\left(P_{\mu}\right)
$$

Since it is true for any $q, t$, we also have that

$$
\begin{aligned}
& \bar{u}_{\mu}\left(P_{\lambda}\left(x ; q^{-1}, t^{-1}\right)\right) \bar{u}_{0}\left(P_{\mu}\left(x ; q^{-1}, t^{-1}\right)\right) \\
& \quad=\bar{u}_{0}\left(P_{\lambda}\left(x ; q^{-1}, t^{-1}\right)\right) \bar{u}_{\lambda}\left(P_{\mu}\left(x ; q^{-1}, t^{-1}\right)\right)
\end{aligned}
$$

and since $P_{\lambda}\left(x ; q^{-1}, t^{-1}\right)=P_{\lambda}(x ; q, t)$ (cf. [Mac95, p.324]), we have another index-variable duality involving the specialization $\bar{u}$, which reads as

$$
\bar{u}_{\mu}\left(P_{\lambda}\right)=\frac{\bar{u}_{0}\left(P_{\lambda}\right)}{\bar{u}_{0}\left(P_{\mu}\right)} \bar{u}_{\lambda}\left(P_{\mu}\right) .
$$

Moreover, using the combinatorial formula for Macdonald polynomials $P$ (see (19)),

$$
\frac{\bar{u}_{0}\left(P_{\lambda}\right)}{\bar{u}_{0}\left(P_{\mu}\right)}=t^{(1-n)|\lambda / \mu|} \frac{u_{0}\left(P_{\lambda}\right)}{u_{0}\left(P_{\mu}\right)} .
$$


As in the proof of Proposition 2.2.17 in [BC14], we can specialize the Pieri rule (for $P$ ) as

$$
\bar{u}_{\lambda}\left(g_{m}(x ; q, t)\right) \bar{u}_{\mu}\left(P_{\lambda}\right)=\sum_{\nu \succ \mu:|v / \mu|=m} \frac{\bar{u}_{0}\left(P_{\nu}\right)}{\bar{u}_{0}\left(P_{\mu}\right)} \varphi_{\nu / \mu} \bar{u}_{\nu}\left(P_{\lambda}\right) .
$$

We need to show that the application of $\bar{u}_{\mu}$ to the right-hand side of (53) equals the application of $\bar{u}_{\mu}$ to the left-hand side. Using (54) and (55), applying $\bar{u}_{\mu}$ to the right-hand side of (53) yields

$$
\sum_{\nu \succ \mu} z^{|v / \mu|} t^{(n-1)|v / \mu|} \frac{\bar{u}_{0}\left(P_{v}\right)}{\bar{u}_{0}\left(P_{\mu}\right)} \varphi_{\nu / \mu} \bar{u}_{\nu}\left(P_{\lambda}\right) .
$$

On the other hand, the application of $\bar{u}_{\mu}$ to the left-hand side of (53) is

$$
\bar{u}_{\mu}\left(\sum_{\eta} z^{|\eta|} h_{\eta}\left(x_{1}^{-1}, \ldots, x_{n}^{-1} ; q, t\right)\left(T_{q^{-1}, x_{i}}\right)^{\eta_{i}} P_{\lambda}(x)\right) .
$$

If $\eta_{i}=v_{i}-\mu_{i}$ for all $i$, then $\bar{u}_{\mu}\left(\left(T_{q^{-1}, x_{i}}\right)^{\eta_{i}} P_{\lambda}\right)=\bar{u}_{v}\left(P_{\lambda}\right)$. So, it is enough to show that for $\eta_{i}=v_{i}-\mu_{i}$,

$$
\bar{u}_{\mu}\left(h_{\eta}\left(x_{1}^{-1}, \ldots, x_{n}^{-1} ; q, t\right)\right)=\frac{\bar{u}_{0}\left(P_{v}\right)}{\bar{u}_{0}\left(P_{\mu}\right)} \varphi_{\nu / \mu} t^{(n-1)|v / \mu|} .
$$

This holds true since it is shown in the proof of Proposition 2.2.17 in [BC14] that

$$
u_{\mu}\left(h_{\eta}\left(x_{1}, \ldots, x_{n} ; q, t\right)\right)=\frac{u_{0}\left(P_{\nu}\right)}{u_{0}\left(P_{\mu}\right)} \varphi_{\nu / \mu} .
$$

Step 2: Let us extend the result to any $x$. Let us denote by $\left[z^{m}\right] \overline{\mathbf{N}}_{n}^{z}$ the operator corresponding to the $z^{m}$ coefficient, that is,

$$
\left[z^{m}\right] \overline{\mathbf{N}}_{n}^{z}=\sum_{\eta_{1}, \ldots, \eta_{n} \geqslant 0,|\eta|=m} h_{\eta}\left(x_{1}^{-1}, \ldots, x_{n}^{-1} ; q, t\right) \prod_{i=1}^{n}\left(\mathbf{T}_{q^{-1}, x_{i}}\right)^{\eta_{i}} .
$$

We have shown that for any partition $\mu$,

$$
\bar{u}_{\mu}\left(\left[z^{m}\right] \overline{\mathbf{N}}_{n}^{z} P_{\lambda}(x)\right)=g_{m}\left(q^{-\lambda_{1}} t^{0}, \ldots, q^{-\lambda_{n}} t^{n-1} ; q, t\right) \bar{u}_{\mu}\left(P_{\lambda}(x)\right) .
$$

By definition $\left[z^{m}\right] \overline{\mathbf{N}}_{n}^{z} P_{\lambda}(x)$ is a finite sum of rational functions, so it is a rational function. Let us fix $x_{2}=q^{-\mu_{2}} t^{2-n}, \ldots, x_{n}=q^{-\mu_{n}} t^{0}$ for some integers $\mu_{2} \geqslant$ $\cdots \geqslant \mu_{n}$. The following equality of rational functions in the variable $X$,

$$
\left[z^{m}\right] \overline{\mathbf{N}}_{n}^{z} P_{\lambda}\left(X, q^{-\mu_{2}} t^{2-n}, \ldots, q^{-\mu_{n}} t^{0}\right)
$$




$$
=g_{m}\left(q^{-\lambda_{1}} t^{0}, \ldots, q^{-\lambda_{n}} t^{n-1} ; q, t\right) P_{\lambda}\left(X, q^{-\mu_{2}} t^{2-n}, \ldots, q^{-\mu_{n}} t^{0}\right)
$$

is satisfied for any $X$ of the form $q^{-\mu_{1}} t^{1-n}$, where $\mu_{1}$ is a nonnegative integer such that $\mu_{1} \geqslant \mu_{2}$; hence (56) is true as an equality between rational functions in the variable $X$. We may iterate this procedure for each variable $x_{2}, \ldots, x_{n}$ in this order, and we obtain that

$$
\left[z^{m}\right] \overline{\mathbf{N}}_{n}^{z} P_{\lambda}(x)=g_{m}\left(q^{-\lambda_{1}} t^{0}, \ldots, q^{-\lambda_{n}} t^{n-1} ; q, t\right) P_{\lambda}(x)
$$

holds as an identity of rational functions in the variables $x_{1}, \ldots, x_{n}$. Thus, we have established (54) as a formal power series in the variable $z$.

Step 3: When $z$ is such that for all $i=1, \ldots n,\left|z q^{-\lambda_{i}} t^{i-1}\right|<1$, the expansion (55) is absolutely convergent. Alternatively, this can also be seen by expanding $\prod_{i=1}^{n} \frac{\left(q^{-\lambda_{i}} t^{i} z\right)_{\infty}}{\left(q^{-i_{i}} t^{i-1} z\right)_{\infty}}$ using the $q$-binomial theorem (12). Since we have already established (54) as a formal power series, the identity holds as a numeric identity for any $z$ such that for all $i=1, \ldots n,\left|z q^{-\lambda_{i}} t^{i-1}\right|<1$.

3.4. $(\boldsymbol{q}, \boldsymbol{t})$-Laplace transforms. In this section, we consider the half-space Macdonald measure with specializations $\rho=\left(a_{1}, \ldots, a_{n}\right)$ for $a_{i} \in(0,1)$ and $\rho^{\swarrow}=\rho$, where $\rho$ is a Macdonald nonnegative specialization of the form $\rho=\rho(\alpha, \beta, \gamma)$ as in Section 2.2.4. We also assume that $\Pi(\vec{a} ; \rho) \Phi(\vec{a})$ can be expanded via the generalized Littlewood identity (30). The observable of the half-space Macdonald measure appearing in Theorem 3.12 can be understood as a $q, t$ analogue of a Laplace transform formula, coming from the properties of the Noumi operator $\mathbf{N}_{n}^{z}$. Actually, in the limit $t=0, q \rightarrow 1$, this becomes exactly a Laplace transform of the rescaled smallest coordinate of the half-space Macdonald random partition (see Section 6.2).

Definition 3.11. For $r \in \mathbb{R}$, we define the contour $\mathcal{D}_{r}$ to be the vertical line $r+\mathbf{i} \mathbb{R}$, oriented from bottom to top.

TheOREM 3.12. Let $z \in \mathbb{C} \backslash \mathbb{R}_{>0}$. Assume the following:

(i) The parameters $a_{1}, \ldots, a_{n} \in(0,1)$ are chosen such that for all $i, j$, $\left|t a_{i} / a_{j}\right|<1$ and $\left|q a_{i} / a_{j}\right|<1$.

(ii) $R \in(0,1)$ is chosen such that $0<q^{R}<a_{i} / a_{j}$ for all $i, j$.

Then we have

$$
\mathbb{E}_{\left(a_{1}, \ldots, a_{n}\right), \rho}^{q, t}\left[\prod_{i=1}^{n} \frac{\left(q^{\lambda_{i}} t^{n-i+1} z\right)_{\infty}}{\left(q^{\lambda_{i}} t^{n-i} z\right)_{\infty}}\right]
$$




$$
\begin{aligned}
= & \sum_{k=0}^{n} \frac{1}{k !} \int_{\mathcal{D}_{R}} \frac{d s_{1}}{2 \mathbf{i} \pi} \cdots \int_{\mathcal{D}_{R}} \frac{d s_{k}}{2 \mathbf{i} \pi} \oint \frac{d w_{1}}{2 \mathbf{i} \pi} \cdots \oint \frac{d w_{k}}{2 \mathbf{i} \pi} \mathcal{A}_{\vec{s}}^{q, t}(\vec{w}) \\
& \times \prod_{i=1}^{k} \Gamma\left(-s_{i}\right) \Gamma\left(1+s_{i}\right) \prod_{i=1}^{k} \frac{\mathcal{G}^{q, t}\left(w_{i}\right)}{\mathcal{G}^{q, t}\left(q^{s_{i}} w_{i}\right)} \frac{\phi\left(w_{i}^{2}\right)(-z)^{s_{i}}}{\phi\left(q^{s_{i}} w_{i}^{2}\right)\left(q^{s_{i}}-1\right) w_{i}}
\end{aligned}
$$

where the integration contours for the variables $w_{i}$ enclose all the $a_{i}$ and no other singularity; we have used the shorthand notations

$$
\mathcal{A}_{\vec{s}}^{q, t}(\vec{w}):=\prod_{1 \leqslant i<j \leqslant k} \frac{\left(q^{s_{j}} w_{j}-q^{s_{i}} w_{i}\right)\left(w_{i}-w_{j}\right) \phi\left(q^{s_{i}+s_{j}} w_{i} w_{j}\right) \phi\left(w_{i} w_{j}\right)}{\left(q_{j}^{s_{i}}\right)\left(q^{s_{j}} w_{j}-w_{i}\right) \phi\left(q^{s_{i}} w_{i} w_{j}\right) \phi\left(q^{s_{j}} w_{j} w_{i}\right)}
$$

and

$$
\mathcal{G}^{q, t}(w)=\prod_{j=1}^{n} \frac{\phi\left(w / a_{j}\right)}{\phi\left(w a_{j}\right)} \frac{1}{\Pi(w ; \rho)}
$$

This theorem is the half-space analogue of [BCGS16, Theorem 4.8], which deals with full-space Macdonald processes.

REMARK 3.13. We can use variables $\left(u_{1}, \ldots, u_{2 k}\right):=\left(w_{1}^{-1}, q^{v_{1}} w_{1}, \ldots, w_{k}^{-1}\right.$, $\left.q^{v_{k}} w_{k}\right)$ so that

$$
\begin{aligned}
\mathcal{A}_{v}^{q, t}(\vec{w}) & =\prod_{1 \leqslant i<j \leqslant 2 k} \frac{u_{j}-u_{i}}{1-u_{i} u_{j}} \prod_{1 \leqslant i<j \leqslant k} \frac{f\left(q^{v_{i}+v_{j}} w_{i} w_{j}\right) f\left(w_{i} w_{j}\right)}{f\left(q^{v_{i}} w_{i} w_{j}\right) f\left(q^{v_{j}} w_{j} w_{i}\right)} \prod_{i=1}^{k} \frac{1-q^{v_{i}}}{q^{v_{i}} w_{i}-w_{i}^{-1}} \\
& =\operatorname{Pf}\left[\frac{u_{j}-u_{i}}{1-u_{i} u_{j}}\right] \prod_{1 \leqslant i<j \leqslant k} \frac{f\left(q^{v_{i}+v_{j}} w_{i} w_{j}\right) f\left(w_{i} w_{j}\right)}{f\left(q^{v_{i}} w_{i} w_{j}\right) f\left(q^{v_{j}} w_{j} w_{i}\right)} \prod_{i=1}^{k} \frac{1-q^{v_{i}}}{q^{v_{i}} w_{i}-w_{i}^{-1}},
\end{aligned}
$$

where $\phi(u)=(t u)_{\infty} /(u)_{\infty}$ and $f(u)=(t u)_{\infty} /(q u)_{\infty}$ as before, and we have used Schur's Pfaffian identity (176). If $q=t$, the function $f$ is constant, and the whole integrand in (65) can be written as a Pfaffian. This is coherent with the fact that the Pfaffian Schur process determines a Pfaffian point process - see [BR05, Gho17a].

Proof of Theorem 3.12. For $|z|<1$, the result follows from the combination of Proposition 3.14, Proposition 3.15 and Lemma 3.17.

Once the result is established for $|z|<1$, one can analytically continue to any $z \in \mathbb{C} \backslash \mathbb{R}_{>0}$ (the reason why both sides are analytic is similar to the proof of [BC14, Theorem 3.2.11]). 
PROPOSITION 3.14. As a formal series in $z$, and as a numeric equality for $|z|<$ 1, we have

$$
\begin{aligned}
& \mathbb{E}_{\left(a_{1}, \ldots, a_{n}\right), \rho}^{q, t}\left[\prod_{i=1}^{n} \frac{\left(q^{\lambda_{i}} t^{n-i+1} z\right)_{\infty}}{\left(q^{\lambda_{i}} t^{n-i} z\right)_{\infty}}\right] \\
& \quad=\left.\frac{\mathbf{N}_{n}^{z} \Pi\left(x_{1}, \ldots, x_{n} ; \rho\right) \Phi\left(x_{1}, \ldots, x_{n}\right)}{\Pi\left(x_{1}, \ldots, x_{n} ; \rho\right) \Phi\left(x_{1}, \ldots, x_{n}\right)}\right|_{x_{1}=a_{1}, \ldots, x_{n}=a_{n}}
\end{aligned}
$$

where $\lambda$ is distributed according to the half-space Macdonald measure with specializations $\left(a_{1}, \ldots, a_{n}\right)$ and $\rho$, and $\mathbf{N}_{n}^{z}$ acts on

$$
\left(x_{1}, \ldots, x_{n}\right) \mapsto \Pi\left(x_{1}, \ldots, x_{n} ; \rho\right) \times \Phi\left(x_{1}, \ldots, x_{n}\right)
$$

Proof. We have

$$
\prod_{i=1}^{n} \frac{\left(q^{\lambda_{i}} t^{n-i+1} z\right)_{\infty}}{\left(q^{\lambda_{i}} t^{n-i} z\right)_{\infty}}=\sum_{k=0}^{+\infty} z^{k} g_{k}\left(q^{\lambda_{1}} t^{n-1}, \ldots, q^{\lambda_{n}}\right)
$$

where we recall that $g_{k}=Q_{(k)}$. Thus the formal series on the left-hand side of (62) is by definition such that the coefficient of $z^{k}$ is $\mathbb{E}^{q, t}\left[g_{k}\left(q^{\lambda_{1}} t^{n-1}, \ldots\right.\right.$, $\left.\left.q^{\lambda_{n}}\right)\right]$. Since $g_{k}\left(q^{\lambda_{1}} t^{n-1}, \ldots, q^{\lambda_{n}}\right)$ is bounded by $g_{k}(1, \ldots, 1)$, it is absolutely integrable with respect to the half-space Macdonald measure. One obtains (62) by multiplying both sides of Noumi's eigenrelation (50) by $\mathcal{E}_{\lambda}(\rho)$ and summing over $\lambda$. Moreover, the series expansion is absolutely convergent when $|z|<$ 1.

Let us now examine the quantity on the right-hand side of (62).

PROPOSITION 3.15. Consider a formal variable $z$, a function $H\left(u_{1}, \ldots, u_{n}\right)=$ $\prod_{i=1}^{n} h\left(u_{i}\right)$ with $h$ being a meromorphic function whose poles are away from the $a_{i}$ 's and $\Phi\left(u_{1}, \ldots, u_{n}\right)=\prod_{i<j} \phi\left(u_{i} u_{j}\right)$ with $\phi(u)=(t u)_{\infty} /(u)_{\infty}$. Then

$$
\begin{aligned}
& \left.\frac{\mathbf{N}_{n}^{z} H \Phi\left(x_{1}, \ldots, x_{n}\right)}{H \Phi\left(x_{1}, \ldots, x_{n}\right)}\right|_{x_{1}=a_{1}, \ldots, x_{n}=a_{n}} \\
& =\sum_{k=0}^{n} \frac{1}{k !} \sum_{v_{1}, \ldots, v_{k}=1}^{\infty} \oint \frac{d w_{1}}{2 \mathbf{i} \pi} \cdots \oint \frac{d w_{k}}{2 \mathbf{i} \pi} \\
& \quad \times \mathcal{A}_{v}^{q, t}(\vec{w}) \prod_{i=1}^{k} \frac{\mathcal{H}_{n}^{q, t}\left(w_{i}\right)}{\mathcal{H}_{n}^{q, t}\left(q^{v_{i}} w_{i}\right)} \frac{\phi\left(w_{i}^{2}\right) z^{v_{i}}}{\phi\left(q^{v_{i}} w_{i}^{2}\right)\left(q^{v_{i}}-1\right) w_{i}},
\end{aligned}
$$


where the (positively oriented) integration contours enclose all the $a_{i}$ 's and no other singularity, and

$$
\mathcal{H}^{q, t}(w)=\prod_{j=1}^{n} \frac{\phi\left(w / a_{j}\right)}{\phi\left(w a_{j}\right)} \frac{1}{h(w)}
$$

Proof. Let us denote

$$
\mathbf{N}_{n, \eta}^{z}=z^{|\eta|} \prod_{i<j} \frac{q^{\eta_{j}} x_{j}-q^{\eta_{i}} x_{i}}{x_{j}-x_{i}} \prod_{i, j} \frac{\left(t x_{i} / x_{j}\right)_{\eta_{i}}}{\left(q x_{i} / x_{j}\right)_{\eta_{i}}} \prod_{i=1}^{n}\left(\mathbf{T}_{q, x_{i}}\right)^{\eta_{i}}
$$

Observe that

$$
\mathbf{N}_{n}^{z}=\sum_{k=0}^{n} \sum_{v_{1}, \ldots, v_{k}=1}^{\infty} \frac{n !}{(n-k) ! k !} \frac{1}{n !} \sum_{\sigma \in \mathcal{S}_{n}} \mathbf{N}_{n, \sigma(v)}^{u}
$$

where on the right-hand side $v=\left(v_{1}, \ldots, v_{k}, 0 \ldots, 0\right)$ and $\sigma \in \mathcal{S}_{n}$ acts by permuting the coordinates of $v$. Hence the proof.

LEMMA 3.16. Assume $v_{1}, \ldots, v_{k} \geqslant 1$ and $v_{k+1}=\cdots=v_{n}=0$. Under the hypotheses of Proposition 3.15, we have

$$
\begin{aligned}
& \left.\frac{1}{(n-k) !} \sum_{\sigma \in \mathcal{S}_{n}} \frac{\mathbf{N}_{n, \sigma(v)}^{z} H \Phi\left(x_{1}, \ldots, x_{n}\right)}{H \Phi\left(x_{1}, \ldots, x_{n}\right)}\right|_{x_{1}=a_{1}, \ldots, x_{n}=a_{n}} \\
& =\oint \frac{d w_{1}}{2 \mathbf{i} \pi} \cdots \oint \frac{d w_{k}}{2 \mathbf{i} \pi} \\
& \quad \times \prod_{1 \leqslant i<j \leqslant k} \frac{\left(q^{v_{j}} w_{j}-q^{v_{i}} w_{i}\right)\left(w_{i}-w_{j}\right) \phi\left(q^{v_{i}+v_{j}} w_{i} w_{j}\right) \phi\left(w_{i} w_{j}\right)}{\left(q^{v_{i}}-w_{j}\right)\left(q^{v_{j}} w_{j}-w_{i}\right) \phi\left(q^{v_{i}} w_{i} w_{j}\right) \phi\left(q^{v_{j}} w_{j} w_{i}\right)} \\
& \quad \times \prod_{i=1}^{k}\left(\prod_{j=1}^{n} \frac{\phi\left(w_{i} / a_{j}\right) \phi\left(q^{v_{i}} w_{i} a_{j}\right)}{\phi\left(q^{v_{i}} w_{i} / a_{j}\right) \phi\left(w_{i} a_{j}\right)}\right) \frac{\phi\left(w_{i}^{2}\right) h\left(q^{v_{1}} w_{i}\right) z^{v_{i}}}{\phi\left(q^{v_{i}} w_{i}^{2}\right) h\left(w_{i}\right)\left(q^{v_{i}}-1\right) w_{i}}
\end{aligned}
$$

where the contours are small positively oriented circles enclosing $a_{i}$ 's and no other singularity.

Proof. The proof is modeled after [BCGS16, Lemma 4.12]. We have

$$
\prod_{i=1}^{n}\left(T_{q, x_{i}}\right)^{v_{i}} H \Phi(\vec{x})=\prod_{i=1}^{n} h\left(q^{v_{i}} x_{i}\right) \prod_{i<j} \phi\left(q^{v_{i}+v_{j}} x_{i} x_{j}\right) .
$$


The left-hand side in (65) equals

$$
\begin{aligned}
& \frac{1}{(n-k) !} \sum_{\sigma \in \mathcal{S}_{n}} z^{|\nu|} \prod_{i<j} \frac{q^{\nu_{j}} a_{\sigma(j)}-q^{\nu_{i}} a_{\sigma(i)}}{a_{\sigma(j)}-a_{\sigma(i)}} \prod_{i, j=1}^{n} \frac{\left(t a_{\sigma(i)} / a_{\sigma(j)}\right)_{v_{i}}}{\left(q a_{\sigma(i)} / a_{\sigma(j)}\right)_{\nu_{i}}} \\
& \times \quad \prod_{i=1}^{n} \frac{h\left(q^{\nu_{i}} a_{\sigma(i)}\right)}{h\left(a_{\sigma(i)}\right)} \prod_{i<j} \frac{\phi\left(q^{\nu_{i}+v_{j}} a_{\sigma(i)} a_{\sigma(j)}\right)}{\phi\left(a_{\sigma(i)} a_{\sigma(j)}\right)}
\end{aligned}
$$

because permuting $v_{i}$ is equivalent to permuting $a_{i}$. Since $v_{k+1}=\cdots=v_{n}=0$, the summand is invariant with respect to permutation of the $\left\{v_{i}\right\}_{i>k}$. Hence one can absorb the factor $1 /(n-k)$ ! and sum on permutations in $\mathcal{S}_{n}^{k}:=$ $\left\{\sigma \in \mathcal{S}_{n}: \sigma(1+k)<\cdots<\sigma(n)\right\}$. Thus, the left-hand side in (65) equals

$$
\begin{aligned}
& \sum_{\substack{\sigma \in \mathcal{S}_{n}^{k} \\
w_{i}=a_{\sigma(i)} \\
\forall 1 \leqslant i \leqslant k}}^{\operatorname{Subs}}\left\{\prod_{i<j} \frac{q^{v_{j}} w_{j}-q^{v_{i}} w_{i}}{w_{j}-w_{i}} \prod_{i=1}^{k} \prod_{j=k+1}^{n} \frac{a_{\sigma(j)}-q^{v_{i}} w_{i}}{a_{\sigma(j)}-w_{i}} \prod_{i=1}^{k} \prod_{j=1}^{n} \frac{\left(t w_{i} / a_{j}\right)_{v_{i}}}{\left(q w_{i} / a_{j}\right)_{v_{i}}}\right. \\
& \quad \times \prod_{i=1}^{n} \frac{h\left(q^{v_{i}} w_{i}\right)}{h\left(w_{i}\right)} \prod_{1 \leqslant i<j \leqslant k} \frac{\phi\left(q^{v_{i}+v_{j}} w_{i} w_{j}\right)}{\phi\left(w_{i} w_{j}\right)} \\
& \left.\quad \times \prod_{i=1}^{k} \prod_{j=1}^{n} \frac{\phi\left(q^{v_{i}} w_{i} a_{j}\right)}{\phi\left(w_{i} a_{j}\right)} \prod_{i=1}^{k} \prod_{j=1}^{k} \frac{\phi\left(w_{i} w_{j}\right)}{\phi\left(q^{v_{i}} w_{i} w_{j}\right)}\right\}
\end{aligned}
$$

Note that we have

$$
\begin{aligned}
& \underset{\substack{w_{i}=a_{\sigma(i)} \\
\forall 1 \leqslant i \leqslant k}}{\operatorname{Subs}}\left\{\prod_{i=1}^{k} \prod_{j=k+1}^{n} \frac{a_{\sigma(j)}-q^{v_{i}} w_{i}}{a_{\sigma(j)}-w_{i}}\right\} \\
& \quad=\underset{\substack{w_{i}=a_{\sigma(i)} \\
\forall 1 \leqslant i \leqslant k}}{\operatorname{Res}}\left\{\prod_{i=1}^{k} \prod_{j=1}^{n} \frac{q^{v_{i}} w_{i}-a_{j}}{w_{i}-a_{j}} \prod_{i, j=1}^{k} \frac{1}{q^{v_{i}} w_{i}-w_{j}} \prod_{\substack{1 \leqslant i \neq j \leqslant k \\
1}}\left(w_{i}-w_{j}\right)\right\},
\end{aligned}
$$

where the notation $\operatorname{Res}_{\substack{w_{i} \leqslant i \leqslant k \\ w_{i}=x_{i}}}\{\cdot\}$ denotes the residue of the function inside brackets at $w_{1}=x_{1}, \ldots, w_{k}=x_{k}$. This implies that

$$
\begin{aligned}
(66)= & \oint \frac{d w_{1}}{2 \mathbf{i} \pi} \cdots \oint \frac{d w_{k}}{2 \mathbf{i} \pi} \prod_{i<j} \frac{q^{v_{j}} w_{j}-q^{v_{i}} w_{i}}{w_{j}-w_{i}} \\
& \times \prod_{i=1}^{k} \prod_{j=1}^{n} \frac{q^{v_{i}} w_{i}-a_{j}}{w_{i}-a_{j}} \prod_{i, j=1}^{k} \frac{1}{q^{v_{i}} w_{i}-w_{j}} \prod_{i \neq j=1}^{k}\left(w_{i}-w_{j}\right) \\
& \times \prod_{i=1}^{k} \prod_{j=1}^{n} \frac{\left(t w_{i} / a_{j}\right)_{v_{i}}}{\left(q w_{i} / a_{j}\right)_{v_{i}}} \prod_{i=1}^{n} \frac{h\left(q^{v_{i}} w_{i}\right)}{h\left(w_{i}\right)} \prod_{1 \leqslant i<j \leqslant k} \frac{\phi\left(q^{v_{i}+v_{j}} w_{i} w_{j}\right)}{\phi\left(w_{i} w_{j}\right)}
\end{aligned}
$$




$$
\times \prod_{i=1}^{k} \prod_{j=1}^{n} \frac{\phi\left(q^{v_{i}} w_{i} a_{j}\right)}{\phi\left(w_{i} a_{j}\right)} \prod_{i=1}^{k} \prod_{j=1}^{k} \frac{\phi\left(w_{i} w_{j}\right)}{\phi\left(q^{v_{i}} w_{i} w_{j}\right)}
$$

where the contours enclose $a_{i}$ 's and no other singularity. Indeed, the integral of the right-hand side can be evaluated by summing all residues at $\left(w_{i}\right)_{1 \leqslant i \leqslant k}=$ $\left(a_{p(i)}\right)_{1 \leqslant i \leqslant k}$, for all functions $p:\{1, \ldots, k\} \rightarrow\{1, \ldots, n\}$, and it is clear that the residue is zero when $p$ is not injective because of the product of $\left(w_{i}-w_{j}\right)$. Hence, the integral is a sum of residues at $w_{i}=a_{\sigma(i)}$ over all $\sigma \in \mathcal{S}_{n}^{k}$. Finally, one has

$$
\frac{q^{v_{i}} w_{i}-a_{j}}{w_{i}-a_{j}} \frac{\left(t w_{i} / a_{j}\right)_{v_{i}}}{\left(q w_{i} / a_{j}\right)_{v_{i}}}=\frac{\phi\left(w_{i} / a_{j}\right)}{\phi\left(q^{v_{i}} w_{i} / a_{j}\right)},
$$

so that the integrand can be arranged to match with the right-hand side of (65).

Let us finish the proof of Proposition 3.15. Recalling the notations in (58) and (59), the application of Lemma 3.16 in each term of the sum in (64) yields the desired result. Furthermore, if $|z|<1$, all sums are absolutely convergent so that (63) holds as a numeric equality.

The expansion on the right of (63) is not exactly as in Theorem 3.12. In order to replace the discrete sums over $v_{i}$ in Proposition 3.15 by contour integrals over vertical contours, we use the following lemma.

LeMmA 3.17. Let $a \in(0,1)$ and $h$ be a holomorphic function on $\{z \in \mathbb{C}$ : $\mathfrak{R e}[z] \geqslant a\}$ such that there exists a constant $C>0$ with

$$
|h(z)| \leqslant C /|z|^{2}
$$

for any $z$ in $\{z \in \mathbb{C}: \mathfrak{R e}[z] \geqslant a\}$. Then, for $\zeta \in \mathbb{C} \backslash \mathbb{R}_{>0}$, we have

$$
\sum_{k=1}^{\infty} h(k) \zeta^{k}=\int_{\mathcal{D}_{a}} \frac{d s}{2 \mathbf{i} \pi}(-\zeta)^{s} \Gamma(-s) \Gamma(1+s) h(s),
$$

whenever both sides are absolutely convergent (recall that $\mathcal{D}_{a}$ is oriented upwards).

Proof. It is clear by the residue theorem that

$$
\sum_{k=1}^{\infty} h(k) \zeta^{k}=\int \frac{d s}{2 \mathbf{i} \pi}(-\zeta)^{s} \Gamma(-s) \Gamma(1+s) h(s),
$$


where the contour is chosen so as to enclose all the positive integers and no other singularity, for example, the union of small positively oriented circles around each positive integer. Using the fact that $h$ is holomorphic, and using the decay bound, one can deform the integration contour to be $\mathcal{D}_{a}$ without changing the value of the integral.

To conclude the proof of Theorem 3.12, we need to check that the hypotheses of Lemma 3.17 are satisfied for its $k$-fold application in the $k$ th term of (63) for all $k \geqslant 1$. On the right-hand side of (57), the integrand in each of the variables $s_{i}$ can be represented as $\Gamma\left(-s_{i}\right) \Gamma\left(1+s_{i}\right)(-\zeta)^{s_{i}} g\left(q^{s_{i}}\right)$, where the function $g$ is an analytic function with isolated singularities. One may inspect all of these singularities one by one and check that for a specialization $\rho=\rho(\alpha, \beta, \gamma)$ with $\alpha_{i} \in(0,1)$ and assuming that the variables $w_{i}$ are integrated along contours very close to the $a_{i}$, there are no singularities in $s_{i}$ lying on the right of $\mathcal{D}_{R}$. Then, since $q^{s}$ stays bounded for $s$ on the right of $\mathcal{D}_{R}, g\left(q^{s}\right)$ stays bounded as well. For $z$ such that $|z|<1$, the integrand satisfies the decay bound of Lemma 3.17. Thus, we have established Theorem 3.12 for $|z|<1$ and it can be then analytically continued to $z \in \mathbb{C} \backslash \mathbb{R}_{>0}$. This completes the proof of Theorem 3.12.

We now seek to prove an analogous result using the operator $\overline{\mathbf{N}}_{z}^{n}$ instead of $\mathbf{N}_{z}^{n}$.

Definition 3.18. We define for $z \in \mathbb{C} \backslash \mathbb{R}_{>0}$

$$
\begin{aligned}
& E_{R}^{z}\left(a_{1}, \ldots, a_{n} ; \rho\right):=\sum_{k=0}^{n} \frac{1}{k !} \int_{\mathcal{D}_{R}} \frac{d s_{1}}{2 \mathbf{i} \pi} \cdots \int_{\mathcal{D}_{R}} \frac{d s_{k}}{2 \mathbf{i} \pi} \oint \frac{d w_{1}}{2 \mathbf{i} \pi} \cdots \oint \frac{d w_{k}}{2 \mathbf{i} \pi} \\
& \quad \times \mathcal{B}_{\vec{s}}^{q, t}(\vec{w}) \prod_{i=1}^{k} \Gamma\left(-s_{i}\right) \Gamma\left(1+s_{i}\right) \frac{\overline{\mathcal{G}}^{q, t}\left(w_{i}\right)}{\overline{\mathcal{G}}^{q, t}\left(q^{-s_{i}} w_{i}\right)} \frac{\phi\left(w_{i}^{2}\right)(-z)^{s_{i}}}{\phi\left(q^{-s_{i}} w_{i}^{2}\right)\left(1-q^{s_{i}}\right) w_{i}}
\end{aligned}
$$

where the $w$ contours are small positively oriented circles enclosing $a_{i}$ and no other singularity,

$$
\mathcal{B}_{\vec{s}}^{q, t}(\vec{w}):=\prod_{1 \leqslant i<j \leqslant k} \frac{\left(q^{s_{j}} w_{i}-q^{s_{i}} w_{j}\right)\left(w_{j}-w_{i}\right) \phi\left(q^{-s_{i}-s_{j}} w_{i} w_{j}\right) \phi\left(w_{i} w_{j}\right)}{\left(q^{s_{i}} w_{j}-w_{i}\right)\left(q^{s_{j}} w_{i}-w_{j}\right) \phi\left(q^{-s_{i}} w_{i} w_{j}\right) \phi\left(q^{-s_{j}} w_{j} w_{i}\right)},
$$

and

$$
\overline{\mathcal{G}}^{q, t}(w)=\prod_{j=1}^{n} \frac{\phi\left(a_{j} / w\right)}{\phi\left(w a_{j}\right)} \frac{1}{\Pi(w ; \rho)}
$$

REMARK 3.19. Unlike the expansion (57) occurring in Theorem 3.12, in (68) the integrand has many poles in the variables $s_{i}$ lying on the right of the contour 
$\mathcal{D}_{R}$. This means that the integral cannot be turned into a discrete sum using Lemma 3.17.

TheOrem 3.20. Let $z \in \mathbb{C} \backslash \mathbb{R}_{>0}$. Assume the following:

(i) The parameters $a_{1}, \ldots, a_{n} \in(0,1)$ are chosen such that for all $i, j$, $\left|t a_{i} / a_{j}\right|<1,\left|q a_{i} / a_{j}\right|<1$ and $\max \left\{a_{i}\right\}<a_{i} / a_{j}$.

(ii) $R \in(0,1)$ is chosen so that for all $i, j, a_{i}<q^{R}<a_{i} / a_{j}$.

(iii) The specialization $\rho=\rho(\alpha, \beta, \gamma)$ is such that for all $i, j, q^{R}>a_{i} \alpha_{j}$. Then,

$$
\mathbb{E}_{\left(a_{1}, \ldots, a_{n}\right), \rho}^{q, t}\left[\prod_{i=1}^{n} \frac{\left(q^{-\lambda_{i}} t^{i} z\right)_{\infty}}{\left(q^{-\lambda_{i}} t^{i-1} z\right)_{\infty}}\right]=E_{R}^{z}\left(a_{1}, \ldots, a_{n} ; \rho\right)
$$

The expectation on the left-hand side of (70) should again be thought of as a Laplace transform. A full-space analogue of this result could be proved by adapting the proof that we present below to the case of the full-space Macdonald processes. Note that in the $q$-Whittaker case $(t=0)$, a full-space analogue is already available [BCFV15, Theorem 3.3].

REMARK 3.21. It will be useful to note that for fixed $n \geqslant 1$ and $z \in \mathbb{C} \backslash \mathbb{R}_{>0}$ the observable $\prod_{i=1}^{n} \frac{\left(q^{-\lambda_{i}} t^{i} z\right)_{\infty}}{\left(q^{-\lambda_{i}} t^{i-1} z\right)_{\infty}}$ is bounded as a function of $\lambda$. Indeed, as long as $t \in(0,1)$, the ratio $\frac{\left(q^{-m} t x\right)_{\infty}}{\left(q^{-m} x\right)_{\infty}}$ goes to zero as $m$ goes to $+\infty$ for any fixed $x \in \mathbb{C} \backslash \mathbb{R}_{>0}$.

REMARK 3.22. Since the left-hand side in (70) is analytic in the parameters $a_{1}$, $\ldots, a_{n}$ and the parameters of the specialization $\rho$, the formula can be extended to a range of parameters forbidden by the hypotheses of Theorem 3.20, at the expense of choosing more complicated contours for the variables $s_{i}$.

REMARK 3.23. The proof of Theorem 3.20 is substantially different-and more difficult - than that of Theorem 3.12. The left-hand side of (70) is by definition

$$
\frac{1}{\Pi(a ; \rho) \Phi(a)} \sum_{\lambda} \mathcal{E}_{\lambda}(\rho) P_{\lambda}(a) \prod_{i=1}^{n} \frac{\left(q^{-\lambda_{i}} t^{i} z\right)_{\infty}}{\left(q^{-\lambda_{i}} t^{i-1} z\right)_{\infty}} .
$$

It is tempting to replace the quantity $P_{\lambda}(a) \prod_{i=1}^{n} \frac{\left(q^{-\lambda_{i}} t^{i}\right)_{\infty}}{\left(q^{-\lambda_{i}} t^{i-1} z\right)_{\infty}}$ above by $\overline{\mathbf{N}}_{n}^{z} P_{\lambda}(a)$ using Proposition 3.10, and exchange the action of the operator $\overline{\mathbf{N}}_{n}^{z}$ with the summation over $\lambda$, to arrive at an analogue of Proposition 3.14. But this exchange 
of summations is forbidden because Proposition 3.10 requires $\left|z q^{-\lambda_{i}} t^{i-1}\right|<1$, which can never be true simultaneously for all $\lambda$.

A natural roundabout way would be to work with formal power series. It is reasonable to expect both sides of (70) to be analytic in the variables $a_{1}, \ldots$, $a_{n}$. For a function $F$ analytic and symmetric in the variables $\left(a_{1}, \ldots, a_{n}\right)=: \vec{a}$, let us denote by $\left[P_{\lambda}(\vec{a})\right]\{F\}$ the coefficient of $P_{\lambda}(\vec{a})$ when the quantity $F$ is expanded in $a_{1}, \ldots, a_{n}$ using the basis of Macdonald polynomials $P_{\lambda}$. Similarly, for a formal power series $F$ in the variable $z$, we likewise denote by $\left[z^{k}\right]\{F\}$ the coefficient of $z^{k}$.

Then, we clearly have

$$
\begin{aligned}
& {\left[z^{k}\right]\left\{\left[P_{\lambda}(\vec{a})\right]\left\{\mathbb{E}_{\left(a_{1}, \ldots, a_{n}\right), \rho}^{q, j}\left[\prod_{i=1}^{n} \frac{\left(q^{-\lambda_{i}} t^{i} z\right)_{\infty}}{\left(q^{-\lambda_{i}} t^{i-1} z\right)_{\infty}}\right]\right\}\right\}} \\
& =\frac{\mathcal{E}_{\lambda}(\rho) g_{k}\left(q^{-\lambda_{1}} t^{0}, \ldots, q^{-\lambda_{n}} t^{n-1}\right)}{\Pi(\vec{a} ; \rho) \Phi(\vec{a})} .
\end{aligned}
$$

On the other hand,

$$
\left[P_{\lambda}(\vec{a})\right]\left\{\left[z^{k}\right]\left\{\overline{\mathbf{N}}_{n}^{z} \Pi(\vec{a} ; \rho) \Phi(\vec{a})\right\}\right\}=\mathcal{E}_{\lambda}(\rho) g_{k}\left(q^{-\lambda_{1}} t^{0}, \ldots, q^{-\lambda_{n}} t^{n-1}\right) .
$$

Unlike the proof of Proposition 3.14, it turns out to be impossible to justify the exchange of summations over $k$ and $\lambda$, and we actually have that in general

$$
\mathbb{E}_{\left(a_{1}, \ldots, a_{n}\right), \rho}^{q, t}\left[\prod_{i=1}^{n} \frac{\left(q^{-\lambda_{i}} t^{i} z\right)_{\infty}}{\left(q^{-\lambda_{i}} t^{i-1} z\right)_{\infty}}\right] \neq \frac{\overline{\mathbf{N}}_{n}^{z} \Pi(\vec{a} ; \rho) \Phi(\vec{a})}{\Pi(\vec{a} ; \rho) \Phi(\vec{a})},
$$

even for $z$ and $\vec{a}$ very close to 0 (this can be checked explicitly when, for example, $n=1$ and $\rho$ is a single variable pure alpha specialization; see also Remark 4.11). To resolve this issue, we will construct another operator $\mathbf{M}_{n}^{z}$, such that

$$
\left[z^{k}\right]\left\{\left[P_{\lambda}(\vec{a})\right]\left\{\mathbf{M}_{n}^{z} \Pi(\vec{a} ; \rho) \Phi(\vec{a})\right\}\right\}=\mathcal{E}_{\lambda}(\rho) g_{k}\left(q^{-\lambda_{1}} t^{0}, \ldots, q^{-\lambda_{n}} t^{n-1}\right) .
$$

The definition of $\mathbf{M}_{n}^{z}$ will be suggested by the analytic continuation of (53) to $z \in \mathbb{C} \backslash \mathbb{R}_{>0}$.

REMARK 3.24. There is another approach to obtain formulas for the observable appearing in (70). We thank an anonymous referee for this suggestion. Acting on the generalized Littlewood identity with the operator $\mathbf{N}_{n}^{z} \prod_{i=1}^{n} \mathbf{T}_{u, x_{i}}$ instead of $\mathbf{N}_{n}^{z}$ as in the proof of Theorem 3.12, we can compute the observable

$$
\mathbb{E}\left[\prod_{i=1}^{n} u^{\lambda_{i}} \frac{\left(q^{\lambda_{i}} t^{n-i+1} z\right)_{\infty}}{\left(q^{\lambda_{i}} t^{n-i} z\right)_{\infty}}\right]
$$


Replacing $(z, u)$ by $\left(\frac{q}{t^{n} z}, t u\right)$, we obtain, after appropriate renormalization, the observable

$$
\mathbb{E}\left[\prod_{i=1}^{n} u^{\lambda_{i}} \frac{\left(q^{-\lambda_{i}} t^{i} z\right)_{\infty}}{\left(q^{-\lambda_{i}} t^{i-1} z\right)_{\infty}}\right] .
$$

This approach has the advantage of avoiding analytic continuations and the introduction of the operator $\mathbf{M}_{n}^{z}$. However, the formula produced by this method is different from the result of Theorem 3.20, and it is not clear how to show the equivalence of both formulas. Because of the substitution $(z, u) \rightarrow\left(\frac{q}{t^{n} z}, t u\right)$, the formula obtained appears singular at $t=0$. It would be interesting to manipulate the formula so as to remove all the singularities at $t=0$. We leave this for future consideration.

\subsection{Proof of Theorem 3.20 .}

Definition 3.25. Let $\mathbb{D}$ be the open unit disk $\mathbb{D}:=\{z \in \mathbb{C}:|z|<1\}$ and $\mathcal{A}^{\mathcal{S}_{n}}\left(\mathbb{D}^{n}\right)$ be the space of analytic symmetric functions $f\left(x_{1}, \ldots, x_{n}\right)$ on $\mathbb{D}^{n}$. (Here we do not mean elements of Sym but analytic functions in $n$ variables that are symmetric in these variables.) Such a function $f$ admits an absolutely convergent expansion in Macdonald symmetric polynomials on $\mathbb{D}^{n}$. More precisely, for all $x=\left(x_{1}, \ldots, x_{n}\right) \in \mathbb{D}^{n}$,

$$
f\left(x_{1}, \ldots, x_{n}\right)=\sum_{\lambda} c_{\lambda}(f) P_{\lambda}(x), \quad \text { with } \sum_{\lambda}\left|c_{\lambda}(f) P_{\lambda}(x)\right|<\infty .
$$

Definition 3.26. We define an operator $\mathbf{M}_{n}^{z}: \mathcal{A}^{\mathcal{S}_{n}}\left(\mathbb{D}^{n}\right) \rightarrow \mathcal{A}^{\mathcal{S}_{n}}\left(\mathbb{D}^{n}\right)$ by

$$
\begin{aligned}
\mathbf{M}_{n}^{z} f\left(x_{1}, \ldots, x_{n}\right)= & \int_{\mathcal{D}_{-\varepsilon}} \frac{d s_{1}}{2 \mathbf{i} \pi} \cdots \int_{\mathcal{D}_{-\varepsilon}} \frac{d s_{n}}{2 \mathbf{i} \pi}(-z)^{s_{1}+\cdots+s_{n}} \prod_{i<j} \frac{q^{s_{j}} x_{i}-q^{s_{i}} x_{j}}{x_{i}-x_{j}} \\
& \times \prod_{i, j} \frac{\left(t x_{i} / x_{j}\right)_{\infty}}{\left(q x_{i} / x_{j}\right)_{\infty}} \frac{\left(q^{s_{j}+1} x_{i} / x_{j}\right)_{\infty}}{\left(t q^{s_{j}} x_{i} / x_{j}\right)_{\infty}} \\
& \times \prod_{i=1}^{n} \Gamma\left(-s_{i}\right) \Gamma\left(1+s_{i}\right) f\left(q^{-s_{1}} x_{1}, \ldots, q^{-s_{n}} x_{n}\right),
\end{aligned}
$$

where $\varepsilon\left(x_{1}, \ldots, x_{n}\right)$ is chosen small enough so that there are no poles of the integrand with real part between $-\varepsilon$ and 0 .

It is not yet clear why $\mathbf{M}_{n}^{z} f$ must belong to $\mathcal{A}^{\mathcal{S}_{n}}\left(\mathbb{D}^{n}\right)$. The next proposition can be interpreted as an analytic continuation of Proposition 3.10. It implies, in particular, that $\mathbf{M}_{n}^{z}$ preserves $\mathcal{A}^{\mathcal{S}_{n}}\left(\mathbb{D}^{n}\right)$. 
Proposition 3.27. For $z \in \mathbb{C} \backslash \mathbb{R}_{>0}$ and $x_{1}, \ldots, x_{n} \in \mathbb{D}$ such that for all $i, j$, $\left|t x_{i} / x_{j}\right|<1$,

$$
\mathbf{M}_{n}^{z} P_{\lambda}\left(x_{1}, \ldots, x_{n}\right)=\prod_{i=1}^{n} \frac{\left(q^{-\lambda_{i}} t^{i} z\right)_{\infty}}{\left(q^{-\lambda_{i}} t^{i-1} z\right)_{\infty}} P_{\lambda}(x)
$$

Proof. When $z$ is such that for all $i=1, \ldots n,\left|z q^{-\lambda_{i}} t^{i-1}\right|<1$ and $x_{1}, \ldots$, $x_{n} \in \mathbb{D}$, (73) is a reformulation of Proposition 3.10 using Mellin-Barnes integrals via Lemma 3.17. (The condition $\left|t x_{i} / x_{j}\right|<1$ ensures that there are no unwanted singularities to the right of the contour $\mathcal{D}_{-\varepsilon}$.)

Both sides of (73) are analytic in $z$ so that one can analytically extend the identity to any $z \in \mathbb{C} \backslash \mathbb{R}_{>0}$. Analyticity is clear for the right-hand side. For the left-hand side, it follows from the exponential decay of $\Gamma\left(-s_{i}\right) \Gamma\left(1+s_{i}\right)$ on the contour $\mathcal{D}_{-\varepsilon}$ so that all integrals are absolutely convergent.

Proposition 3.28. Let $f \in \mathcal{A}^{\mathcal{S}_{n}}\left(\mathbb{D}^{n}\right)$ with $f\left(x_{1}, \ldots, x_{n}\right)=\sum_{\lambda} c_{\lambda}(f) P_{\lambda}(x)$. Then for $x_{1}, \ldots, x_{n} \in(0,1)$ such that for all $i, j,\left|t x_{i} / x_{j}\right|<1$, we have

$$
\mathbf{M}_{n}^{z} f\left(x_{1}, \ldots, x_{n}\right)=\sum_{\lambda} c_{\lambda}(f) P_{\lambda}(x) \prod_{i=1}^{n} \frac{\left(q^{-\lambda_{i}} t^{i} z\right)_{\infty}}{\left(q^{-\lambda_{i}} t^{i-1} z\right)_{\infty}} .
$$

Proof. The operator $\mathbf{M}_{n}^{z}$ is linear on $\mathcal{A}^{\mathcal{S}_{n}}\left(\mathbb{D}^{n}\right)$ so that

$$
\mathbf{M}_{n}^{z} f\left(x_{1}, \ldots, x_{n}\right)=\sum_{\lambda} c_{\lambda}(f) \mathbf{M}_{n}^{z} P_{\lambda}(x),
$$

and one can apply Proposition 3.27 on each summand. When applying the operator $\mathbf{M}_{n}^{z}$, one has to choose the parameter $\varepsilon$ involved in the integration contour in (73) in such a way that for all $i,\left|q^{-\varepsilon} x_{i}\right|<1$.

COROllary 3.29. For $x_{1}, \ldots, x_{n} \in(0,1)$ such that for all $i, j,\left|t x_{i} / x_{j}\right|<1$,

$$
\frac{\mathbf{M}_{n}^{z}(\Pi(x, \rho) \Phi(x))}{\Pi(x, \rho) \Phi(x)}=\mathbb{E}_{\left(a_{1}, \ldots, a_{n}\right), \rho}^{q, t}\left[\prod_{i=1}^{n} \frac{\left(q^{-\lambda_{i}} t^{i} z\right)_{\infty}}{\left(q^{-\lambda_{i}} t^{i-1} z\right)_{\infty}}\right] .
$$

Proof. This is a direct application of Proposition 3.28 with $f(x)=\Pi(x$, $\rho) \Phi(x)$.

To conclude the proof of Theorem 3.20, we need to compute the left-hand side in (74). 
PROPOSITION 3.30. Under the hypotheses of Theorem 3.20,

$$
\frac{\mathbf{M}_{n}^{z}(\Pi(x, \rho) \Phi(x))}{\Pi(x, \rho) \Phi(x)}=E_{R}^{z}\left(a_{1}, \ldots, a_{n} ; \rho\right) .
$$

Proof. By definition,

$$
\begin{aligned}
& \mathbf{M}_{n}^{z}(\Pi(x, \rho) \Phi(x))=\int_{\mathcal{D}_{-\varepsilon}} \frac{d s_{1}}{2 \mathbf{i} \pi} \cdots \int_{\mathcal{D}_{-\varepsilon}} \frac{d s_{n}}{2 \mathbf{i} \pi}(-z)^{s_{1}+\cdots+s_{n}} \\
& \quad \times \prod_{i<j} \frac{q^{s_{j}} x_{i}-q^{s_{i}} x_{j}}{x_{i}-x_{j}} \prod_{i, j} \frac{\left(t x_{i} / x_{j}\right)_{\infty}}{\left(q x_{i} / x_{j}\right)_{\infty}} \frac{\left(q^{s_{j}+1} x_{i} / x_{j}\right)_{\infty}}{\left(t q^{s_{j}} x_{i} / x_{j}\right)_{\infty}} \\
& \quad \times \prod_{i=1}^{n} \Gamma\left(-s_{i}\right) \Gamma\left(1+s_{i}\right) \Pi\left(q^{-s_{1}} x_{1}, \ldots, q^{-s_{n}} x_{n}, \rho\right) \Phi\left(q^{-s_{1}} x_{1}, \ldots, q^{-s_{n}} x_{n}\right) .
\end{aligned}
$$

The first step is to transform this $n$-fold integral into a sum of $k$-fold integrals for $k=1, \ldots, n$ by taking the residues when some of the $s_{i}$ are zero. Let us denote the integrand in (75) by $I_{\vec{s}}^{z}$, where $\vec{s}=\left(s_{1}, \ldots, s_{n}\right)$. Deforming the contours $\mathcal{D}_{-\varepsilon}$ to $\mathcal{D}_{\varepsilon}$ yields

$$
\mathbf{M}_{n}^{z}(\Pi(x, \rho) \Phi(x))=\sum_{k=0}^{n} \int_{\mathcal{D}_{\varepsilon}} \frac{d s_{1}}{2 \mathbf{i} \pi} \cdots \int_{\mathcal{D}_{\varepsilon}} \frac{d s_{k}}{2 \mathbf{i} \pi} \frac{n !}{(n-k) ! k !} \frac{1}{n !} \sum_{\sigma \in \mathcal{S}_{n}} I_{\sigma(\vec{s})}^{z},
$$

where in the $k$ th summand, $\vec{s}=\left(s_{1}, \ldots, s_{k}, 0, \ldots, 0\right)$ and $\sigma$ acts by permuting the coordinates of $\vec{s}$. The only singularity that we cross from $\mathcal{D}_{-\varepsilon}$ to $\mathcal{D}_{\varepsilon}$ is at $s=0$ (it comes from the factor $\Gamma(-s)$ ).

We further deform the integration contour from $\mathcal{D}_{\varepsilon}$ to $\mathcal{D}_{R}$. The next lemma ensures that when $x_{i}$ 's satisfy the same hypotheses as $a_{i}$ in the statement of Theorem 3.20, we do not cross any singularity.

LEMMA 3.31. Under assumptions (i), (ii) and (iii) of Theorem 3.20, the poles in variables $s_{1}, \ldots, s_{n}$ in

$$
\prod_{i, j} \frac{\left(q^{s_{j}+1} a_{i} / a_{j}\right)_{\infty}}{\left(t q^{s_{j}} a_{i} / a_{j}\right)_{\infty}} \Pi\left(q^{-s_{1}} a_{1}, \ldots, q^{-s_{n}} a_{n}, \rho\right) \Phi\left(q^{-s_{1}} a_{1}, \ldots, q^{-s_{n}} a_{n}\right)
$$

do not lie between $\mathcal{D}_{0}$ and $\mathcal{D}_{R}$.

Proof. When $\rho$ is of the form $\rho=\rho(\alpha, \beta, \gamma)$ as in Section 2.2.4, the poles of $\Pi\left(q^{-s_{j}} a_{j} ; \rho\right)$ in the variable $s_{j}$ are the same as those of

$$
\prod_{i} \frac{1}{\left(\alpha_{i} q^{-s_{j}} a_{j}\right)_{\infty}}
$$


Hence, the poles in (77) correspond to the following cases:

(1) $q^{s_{i}+s_{j}}=q^{k} a_{i} a_{j}$ for some integer $k \geqslant 0$ and $1 \leqslant i \neq j \leqslant n$.

(2) $q^{s_{j}}=q^{-k} a_{j} t^{-1} a_{i}^{-1}$ for some integer $k \geqslant 0$ and $1 \leqslant i, j \leqslant n$.

(3) $q^{s_{j}}=\alpha_{i} a_{j} q^{k}$ for some integer $k \geqslant 0,1 \leqslant j \leqslant n$ and any $i$.

Since $q^{R}>a_{i}$ for all $1 \leqslant j \leqslant n$, the poles in case (1) all lie to the right of $\mathcal{D}_{R}$. Since $\left|t a_{i} / a_{j}\right|<1$, we have $1<\left|a_{j} t^{-1} a_{i}^{-1}\right|<t^{-2}$ and the poles in case (2) all have negative real part. Since $q^{R}>a_{j} \alpha_{i}$ for $1 \leqslant j \leqslant n$ and any $i$, the poles in case (3) all lie to the right of $\mathcal{D}_{R}$.

We have arrived at

$$
\frac{\mathbf{M}_{n}^{z}(\Pi(x, \rho) \Phi(x))}{\Pi(x, \rho) \Phi(x)}=\sum_{k=0}^{n} \int_{\mathcal{D}_{R}} \frac{d s_{1}}{2 \mathbf{i} \pi} \cdots \int_{\mathcal{D}_{R}} \frac{d s_{k}}{2 \mathbf{i} \pi} \frac{1}{k !} \frac{1}{(n-k) !} \sum_{\sigma \in \mathcal{S}_{n}} \frac{I_{\sigma(\vec{s})}^{z}}{\Pi(x, \rho) \Phi(x)},
$$

where again $\vec{s}=\left(s_{1}, \ldots, s_{k}, 0, \ldots, 0\right)$.

The last step is to rewrite the sum over permutations in the right-hand side of (78) as some contour integral.

Lemma 3.32. Assume $\mathfrak{R e}\left[s_{1}\right], \ldots, \mathfrak{R e}\left[s_{k}\right]>0$ and $s_{k+1}=\cdots=s_{n}=0$. Then

$$
\begin{aligned}
& \frac{1}{(n-k) !} \sum_{\sigma \in \mathcal{S}_{n}} \frac{I_{\sigma(\vec{s})}^{z}}{\Pi(x, \rho) \Phi(x)} \\
& =\oint \frac{d w_{1}}{2 \mathbf{i} \pi} \cdots \oint \frac{d w_{k}}{2 \mathbf{i} \pi} \\
& \quad \times \mathcal{B}_{\vec{s}}^{q, t}(\vec{w}) \prod_{i=1}^{k} \frac{\mathcal{G}_{n}^{q, t}\left(w_{i}\right)}{\mathcal{G}_{n}^{q, t}\left(q^{-s_{i}} w_{i}\right)} \frac{\phi\left(w_{i}^{2}\right) \Pi\left(q^{-s_{i}} w_{i} ; \rho\right)(-z)^{s_{i}} d w_{i}}{\phi\left(q^{-s_{i}} w_{i}^{2}\right) \Pi\left(w_{i} ; \rho\right)\left(1-q^{s_{i}}\right) w_{i}}
\end{aligned}
$$

where the contours are small positively oriented circles enclosing $x_{i}$ and no other singularity.

Proof. We adapt Lemma 3.16. The left-hand side in (79) equals

$$
\begin{aligned}
& \frac{1}{(n-k) !} \sum_{\sigma \in \mathcal{S}_{n}} z^{s_{1}+\cdots+s_{k}} \prod_{i<j} \frac{q^{s_{j}} x_{\sigma(i)}-q^{s_{i}} x_{\sigma(j)}}{x_{\sigma(i)}-x_{\sigma(j)}} \\
& \quad \times \prod_{i, j=1}^{n} \frac{\left(t x_{\sigma(j)} / x_{\sigma(i)}\right)_{\infty}}{\left(q x_{\sigma(j)} / x_{\sigma(i)}\right)_{\infty}} \frac{\left(q^{s_{i}+1} x_{\sigma(j)} / x_{\sigma(i)}\right)_{\infty}}{\left(t q^{s_{i}} x_{\sigma(j)} / x_{\sigma(i)}\right)_{\infty}}
\end{aligned}
$$




$$
\times \prod_{i=1}^{n} \frac{\Pi\left(q^{-s_{i}} x_{\sigma(i)} ; \rho\right)}{\Pi\left(x_{\sigma(i)} ; \rho\right)} \prod_{i<j} \frac{\phi\left(q^{-s_{i}-s_{j}} x_{\sigma(i)} x_{\sigma(j)}\right)}{\phi\left(x_{\sigma(i)} x_{\sigma(j)}\right)}
$$

Since $s_{k+1}=\cdots=s_{n}=0$, the summand is invariant with respect to permutation of $\left\{s_{i}\right\}_{i>k}$. Hence one can absorb the factor $1 /(n-k)$ ! and sum over permutations in

$$
\mathcal{S}_{n}^{k}:=\left\{\sigma \in \mathcal{S}_{n}: \sigma(1+k)<\cdots<\sigma(n)\right\} .
$$

Thus, the left-hand side in (79) equals

$$
\begin{aligned}
& \sum_{\substack{\sigma \in \mathcal{S}_{n}^{k} \\
\operatorname{Subs}_{i}=x_{\sigma(i)}}}\left\{z^{s_{1}+\cdots+s_{k}} \prod_{i<j} \frac{q^{s_{j}} w_{i}-q^{s_{i}} w_{j}}{w_{i}-w_{j}} \prod_{i=1}^{k} \prod_{j=k+1}^{n} \frac{w_{i}-q^{s_{i}} x_{\sigma(j)}}{w_{i}-x_{\sigma(j)}}\right. \\
& \times \prod_{i=1}^{k} \prod_{j=1}^{n} \frac{\left(t x_{\sigma(j)} / w_{i}\right)_{\infty}}{\left(q x_{\sigma(j)} / w_{i}\right)_{\infty}} \frac{\left(q^{s_{i}+1} x_{\sigma(j)} / w_{i}\right)_{\infty}}{\left(t q^{s_{i}} x_{\sigma(j)} / w_{i}\right)_{\infty}} \\
& \times \prod_{i=1}^{k} \frac{\Pi\left(q^{-s_{i}} w_{i} ; \rho\right)}{\Pi\left(w_{i} ; \rho\right)} \prod_{1 \leqslant i<j \leqslant k} \frac{\phi\left(q^{-s_{i}-s_{j}} w_{i} w_{j}\right)}{\phi\left(w_{i} w_{j}\right)} \\
& \left.\times \prod_{i=1}^{k} \prod_{j=1}^{n} \frac{\phi\left(q^{-s_{i}} w_{i} x_{j}\right)}{\phi\left(w_{i} x_{j}\right)} \prod_{i=1}^{k} \prod_{j=1}^{k} \frac{\phi\left(w_{i} w_{j}\right)}{\phi\left(q^{-s_{i}} w_{i} w_{j}\right)}\right\} \text {. }
\end{aligned}
$$

Note that we have

$$
\begin{aligned}
\underset{\substack{w_{i}=x_{\sigma(i)} \\
\forall 1 \leqslant i \leqslant k}}{\operatorname{Subs}}\left\{\prod_{i=1}^{k} \prod_{j=k+1}^{n} \frac{w_{i}-q^{s_{i}} x_{\sigma(j)}}{w_{i}-x_{\sigma(j)}}\right\} \\
\quad=\underset{\substack{w_{i}=x_{\sigma(i)} \\
\forall 1 \leqslant i \leqslant k}}{\operatorname{Res}}\left\{\prod_{i=1}^{k} \prod_{j=1}^{n} \frac{w_{i}-q^{s_{i}} x_{j}}{w_{i}-x_{j}} \prod_{i, j=1}^{k} \frac{1}{w_{i}-q^{s_{i}} w_{j}} \prod_{i \neq j=1}^{k}\left(w_{i}-w_{j}\right)\right\} .
\end{aligned}
$$

It implies that

$$
\begin{aligned}
(81)= & \oint \frac{d w_{1}}{2 \mathbf{i} \pi} \cdots \oint \frac{d w_{k}}{2 \mathbf{i} \pi} \prod_{i<j} \frac{q^{s_{j}} w_{i}-q^{s_{i}} w_{j}}{w_{i}-w_{j}} \prod_{i=1}^{k} \prod_{j=1}^{n} \frac{w_{i}-q^{s_{i}} x_{j}}{w_{i}-x_{j}} \\
& \times \prod_{i, j=1}^{k} \frac{1}{w_{i}-q^{s_{i}} w_{j}} \prod_{i \neq j=1}^{k}\left(w_{i}-w_{j}\right) \prod_{i=1}^{k} \prod_{j=1}^{n} \frac{\left(t x_{j} / w_{i}\right)_{\infty}}{\left(q x_{j} / w_{i}\right)_{\infty}} \frac{\left(q^{s_{i}+1} x_{j} / w_{i}\right)_{\infty}}{\left(t q^{s_{i}} x_{j} / w_{i}\right)_{\infty}} \\
& \times \prod_{i=1}^{k} \frac{\Pi\left(q^{-s_{i}} w_{i} ; \rho\right)}{\Pi\left(w_{i} ; \rho\right)} \prod_{1 \leqslant i<j \leqslant k} \frac{\phi\left(q^{-s_{i}-s_{j}} w_{i} w_{j}\right)}{\phi\left(w_{i} w_{j}\right)}
\end{aligned}
$$




$$
\times \prod_{i=1}^{k} \prod_{j=1}^{n} \frac{\phi\left(q^{-s_{i}} w_{i} x_{j}\right)}{\phi\left(w_{i} x_{j}\right)} \prod_{i=1}^{k} \prod_{j=1}^{k} \frac{\phi\left(w_{i} w_{j}\right)}{\phi\left(q^{-s_{i}} w_{i} w_{j}\right)}
$$

where the contours enclose $x_{i}$ and no other singularity. The assumption (cf. assumption (ii) in Theorem 3.20) that the real part of the variables $s_{i}$ is such that $\left|q^{s_{i}}\right|<x_{j} / x_{i}$ for all $i, j$ ensures that one can choose these contours as small circles enclosing $x_{i}$. Finally, using the fact that $\phi(u)=(t u)_{\infty} /(u)_{\infty}$, one has

$$
\frac{w_{i}-q^{s_{i}} x_{j}}{w_{i}-x_{j}} \frac{\left(t x_{j} / w_{i}\right)_{\infty}}{\left(q x_{j} / w_{i}\right)_{\infty}} \frac{\left(q^{s_{i}+1} x_{j} / w_{i}\right)_{\infty}}{\left(t q^{s_{i}} x_{j} / w_{i}\right)_{\infty}}=\frac{\phi\left(x_{j} / w_{i}\right)}{\phi\left(q^{s_{i}} x_{j} / w_{i}\right)},
$$

so that the integrand can be arranged to match with the right-hand side of (79).

The application of Lemma 3.32 to each term of the sum in (78) yields the desired formula, which concludes the proof of Proposition 3.30 and Theorem 3.20 .

\section{Half-space $q$-Whittaker processes}

We assume now that $t=0$ and $q \in(0,1)$. We will, however, use the same notations $P, Q, \mathcal{E}$ as before. These $(P$ and $Q$ ) are now called $q$-Whittaker functions [GLO09]. We define the half-space $q$-Whittaker measure $\mathbb{P}_{\rho, \rho}^{q}$ as the measure on partitions $\lambda \in \mathbb{Y}$ such that

$$
\mathbb{P}_{\rho, \rho \swarrow}^{q}(\lambda)=\frac{P_{\lambda}(\rho) \mathcal{E}_{\lambda}\left(\rho^{\swarrow}\right)}{\Pi\left(\rho ; \rho^{\swarrow}\right) \Phi(\rho)},
$$

and denote by $\mathbb{P}_{\omega, \rho}^{q}$, for a sequence of specializations $\rho$, the half-space $q$ Whittaker process, that is, the $t=0$ degeneration of the half-space Macdonald process $\mathbb{P}_{\omega, \rho}^{q, t}$.

4.1. Observables and integral formulas. Consider a $q$-Whittaker measure where $\rho=\left(a_{1}, \ldots, a_{n}\right) \in(0,1)^{n}$, and $\rho^{\swarrow}=\rho(\alpha, \beta, \gamma)$ as defined in Section 2.2.4. We will now degenerate the results from Sections 3.2 and 3.4 to the $q$ Whittaker case. We further assume that the beta component of the specialization $\rho^{\swarrow}$ is trivial (that is, $\beta_{i} \equiv 0$ ), and all the parameters $\alpha_{i}$ are such that $\max \left\{a_{i}\right\} \max \left\{\alpha_{j}\right\}<1$, since this is the case that matters in our applications.

It is convenient to define functions

$$
\mathcal{G}^{q}(w)=e^{-\gamma w} \prod_{j=1}^{n} \frac{\left(w a_{j}\right)_{\infty}}{\left(w / a_{j}\right)_{\infty}} \prod_{j=1}^{\ell}\left(w \alpha_{j}\right)_{\infty} \quad \text { and }
$$




$$
\overline{\mathcal{G}}^{q}(w)=e^{-\gamma w} \prod_{j=1}^{n} \frac{\left(w a_{j}\right)_{\infty}}{\left(a_{j} / w\right)_{\infty}} \prod_{j=1}^{\ell}\left(w \alpha_{j}\right)_{\infty} .
$$

4.1.1. Moment formulas. Let us write explicitly the $q$-Whittaker degeneration of moment formulas from Section 3.2. The moments appearing in Corollary 4.1 can also be written in a different form as in Corollary 4.9 and Corollary 4.4. While both statements could in principle be deduced from Corollary 4.1, it is much simpler to deduce them from the $q$-Laplace transform formulas; hence they will appear in the next section.

COROLLARY 4.1. Under the $q$-Whittaker measure $\mathbb{P}_{\left(a_{1}, \ldots, a_{n}\right),\left(\left(\alpha_{1}, \ldots, \alpha_{\ell}\right), \gamma\right)}^{q}$, the two following moment formulas hold. For any $k \in \mathbb{Z}_{>0}$,

$$
\begin{aligned}
\mathbb{E}^{q}\left[q^{k \lambda_{N}}\right]= & (-1)^{k} \oint \frac{d w_{1}}{2 \mathbf{i} \pi} \cdots \oint \frac{d w_{k}}{2 \mathbf{i} \pi} \prod_{1 \leqslant a<b \leqslant k} \frac{w_{a}-w_{b}}{q^{-1} w_{a}-w_{b}} \frac{1-q w_{a} w_{b}}{1-w_{a} w_{b}} \\
& \times \prod_{m=1}^{k} \frac{1}{1-w_{m}^{2}} \frac{\mathcal{G}^{q}\left(w_{m}\right)}{\mathcal{G}^{q}\left(q w_{m}\right)} \frac{1}{w_{m}},
\end{aligned}
$$

where the positively oriented contours are such that for all $1 \leqslant c \leqslant k$, the contour for $w_{c}$ encloses $\left\{a_{j}\right\}_{1 \leqslant j \leqslant n}$ and $q\left\{w_{c+1}, \ldots, w_{k}\right\}$, and excludes the poles of the integrand at 1 and 0 .

For all $k \in \mathbb{Z}_{>0}$ such that $q^{k}>\left(\max \left\{a_{j}\right\}\right)^{2}$ and $q^{k}>\max \left\{\alpha_{j}\right\} \max \left\{a_{j}\right\}$,

$$
\begin{aligned}
\mathbb{E}^{q}\left[q^{-k \lambda_{1}}\right]= & (-1)^{k} \oint \frac{d w_{1}}{2 \mathbf{i} \pi} \cdots \oint \frac{d w_{k}}{2 \mathbf{i} \pi} \prod_{1 \leqslant a<b \leqslant k} \frac{w_{a}-w_{b}}{q^{-1} w_{a}-w_{b}} \frac{q^{-1}-w_{a} w_{b}}{q^{-2}-w_{a} w_{b}} \\
& \times \prod_{m=1}^{k} \frac{q w_{m}^{2}-1}{q w_{m}^{2}} \frac{\overline{\mathcal{G}}^{q}\left(w_{m}^{-1}\right)}{\overline{\mathcal{G}}^{q}\left(q^{-1} w_{m}^{-1}\right)} \frac{1}{w_{m}},
\end{aligned}
$$

where the positively oriented contours are such that for all $1 \leqslant c \leqslant k$, the contour for $w_{c}$ encloses $\left\{1 / a_{j}\right\}_{1 \leqslant j \leqslant n}$ and $q\left\{w_{c+1}, \ldots, w_{k}\right\}$, and excludes the poles of the integrand at $0, \alpha_{i} / q$ (for $\left.1 \leqslant i \leqslant \ell\right)$ and $a_{j} / q($ for $1 \leqslant j \leqslant n)$.

Proof. This is the $t=0$ degeneration of Proposition 3.5. Note that

$$
\frac{\mathcal{G}^{q}(w)}{\mathcal{G}^{q}(q w)}=\exp ((q-1) \gamma w) \prod_{j=1}^{n}\left(\frac{1-w a_{j}}{1-w / a_{j}}\right) \prod_{i=1}^{\ell}\left(1-\alpha_{i} w\right)
$$


and

$$
\begin{aligned}
\frac{\overline{\mathcal{G}}^{q}\left(w^{-1}\right)}{\overline{\mathcal{G}}^{q}\left(q^{-1} w^{-1}\right)}= & \exp \left(\left(q^{-1}-1\right) \gamma w^{-1}\right) \prod_{j=1}^{n}\left(\frac{q w}{\left(1-w a_{j}\right)\left(q w-a_{j}\right)}\right) \\
& \times \prod_{i=1}^{\ell}\left(\frac{q w}{q w-\alpha_{i}}\right) .
\end{aligned}
$$

COROLLARY 4.2. Under the $q$-Whittaker measure $\mathbb{P}_{\left(a_{1}, \ldots, a_{n}\right),\left(\left(\alpha_{1}, \ldots, \alpha_{\ell}\right), \gamma\right)}^{q}$, the two following formulas hold. For $r \geqslant 1$,

$$
\begin{aligned}
\mathbb{E}^{q}\left[q^{\lambda_{N}+\cdots+\lambda_{N-r+1}}\right]= & \frac{(-1)^{\frac{r(r+1)}{2}}}{r !} \oint \frac{d z_{1}}{2 \mathbf{i} \pi} \cdots \oint \frac{d z_{r}}{2 \mathbf{i} \pi} \prod_{j=1}^{r}\left(\frac{1}{\left(z_{j}\right)^{r}} \frac{1}{1-z_{j}^{2}} \frac{\mathcal{G}^{q}\left(z_{j}\right)}{\mathcal{G}^{q}\left(q z_{j}\right)}\right) \\
& \times \prod_{1 \leqslant i<j \leqslant r}\left(\left(z_{i}-z_{j}\right)^{2} \frac{1-q z_{i} z_{j}}{1-z_{i} z_{j}}\right),
\end{aligned}
$$

where the positively oriented contours encircle $\left\{a_{1}, \ldots, a_{N}\right\}$ and no other singularity of the integrand.

For $r \geqslant 1$, and assuming that $q^{r}>\left(\max \left\{a_{j}\right\}\right)^{2}$ and $q^{r}>\max \left\{\alpha_{j}\right\} \max \left\{a_{j}\right\}$,

$$
\begin{aligned}
\mathbb{E}^{q}\left[q^{-\lambda_{1}-\ldots-\lambda_{r}}\right]= & \frac{(-1)^{\frac{r(r+1)}{2}}}{r !} \oint \frac{d w_{1}}{2 \mathbf{i} \pi} \cdots \oint \frac{d w_{r}}{2 \mathbf{i} \pi} \\
& \times \prod_{j=1}^{r}\left(\frac{1}{\left(w_{i}\right)^{r}} \frac{q w_{j}^{2}-1}{q w_{j}^{2}} \frac{\overline{\mathcal{G}}^{q}\left(w_{j}^{-1}\right)}{\overline{\mathcal{G}}^{q}\left(q^{-1} w_{j}^{-1}\right)}\right) \\
& \times \prod_{1 \leqslant i<j \leqslant r}\left(\left(w_{i}-w_{j}\right)^{2} \frac{q^{2} w_{i} w_{j}-q}{q^{2} w_{i} w_{j}-1}\right),
\end{aligned}
$$

where the positively oriented contours encircle $\left\{1 / a_{1}, \ldots, 1 / a_{N}\right\}$ and no other singularity.

Proof. This is the $t=0$ degeneration of Proposition 3.7.

4.1.2. q-Laplace transform formulas. We consider first the $q$-Laplace transform of $q^{\lambda_{n}}$.

COROllary 4.3. Let $z \in \mathbb{C} \backslash \mathbb{R}_{>0}$. Assume that the parameters $a_{1}, \ldots, a_{n} \in(0$, 1) are chosen so that for all $i, j,\left|q a_{i} / a_{j}\right|<1$, and let $0<R<1$ be such that 
$0<q^{R}<a_{i} / a_{j}$ for all $i, j$. Under the $q$-Whittaker measure $\mathbb{P}_{\left(a_{1}, \ldots, a_{n}\right),\left(\left(\alpha_{1}, \ldots, \alpha_{\ell}\right), \gamma\right)}^{q}$, we have

$$
\begin{aligned}
\mathbb{E}^{q} & {\left[\frac{1}{\left(q^{\lambda_{n}} z\right)_{\infty}}\right]=\sum_{k=0}^{n} \frac{1}{k !} \int_{\mathcal{D}_{R}} \frac{d s_{1}}{2 \mathbf{i} \pi} \cdots \int_{\mathcal{D}_{R}} \frac{d s_{k}}{2 \mathbf{i} \pi} \oint \frac{d w_{1}}{2 \mathbf{i} \pi} \cdots \oint \frac{d w_{k}}{2 \mathbf{i} \pi} } \\
& \times \prod_{1 \leqslant i<j \leqslant k} \frac{\left(q^{s_{j}} w_{j}-q^{s_{i}} w_{i}\right)\left(w_{i}-w_{j}\right)\left(q^{s_{i}} w_{i} w_{j}\right)_{\infty}\left(q^{s_{j}} w_{j} w_{i}\right)_{\infty}}{\left(q^{s_{i}} w_{i}-w_{j}\right)\left(q^{s_{j}} w_{j}-w_{i}\right)\left(q^{s_{i}+s_{j}} w_{i} w_{j}\right)_{\infty}\left(w_{i} w_{j}\right)_{\infty}} \\
& \times \prod_{i=1}^{k}\left[\Gamma\left(-s_{i}\right) \Gamma\left(1+s_{i}\right) \frac{\mathcal{G}^{q}\left(w_{i}\right)}{\mathcal{G}^{q}\left(q^{s_{i}} w_{i}\right)} \frac{\left(q^{s_{i}} w_{i}^{2}\right)_{\infty}(-z)^{s_{i}}}{\left(w_{i}^{2}\right)_{\infty}\left(q^{s_{i}}-1\right) w_{i}}\right],
\end{aligned}
$$

where the positively oriented integration contours for the variables $w_{i}$ enclose all $a_{i}$ and no other singularity.

Proof. This is the $t=0$ degeneration of Theorem 3.12.

It is possible to extract the coefficient of $z^{k}$ in the above formula, which yields the following alternative moment formula.

COROLlaRY 4.4. For any $r \in \mathbb{Z}_{>0}$, under the $q$-Whittaker measure $\mathbb{P}_{\left(a_{1}, \ldots, a_{n}\right),\left(\left(\alpha_{1}, \ldots, \alpha_{\ell}\right), \gamma\right)}^{q}$,

$$
\begin{aligned}
& \mathbb{E}^{q}\left[q^{r \lambda_{n}}\right] \\
& =(q ; q)_{r} \sum_{\substack{\mu \vdash r \\
\mu=1^{m_{1} 2^{m_{2}}} \ldots}} \frac{1}{m_{1} ! m_{2} ! \ldots} \\
& \times \oint \frac{d w_{1}}{2 \mathbf{i} \pi} \cdots \oint \frac{d w_{\ell(\mu)}}{2 \mathbf{i} \pi} \operatorname{det}\left[\frac{1}{w_{i} q^{\mu_{i}}-w_{j}}\right]_{i, j=1}^{\ell(\mu)} \\
& \times \prod_{1 \leqslant a<b \leqslant \ell(\mu)} \frac{\left(q^{\mu_{a}} w_{a} w_{b}\right)_{\infty}\left(q^{\mu_{b}} w_{a} w_{b}\right)_{\infty}}{\left(w_{a} w_{b}\right)_{\infty}\left(q^{\mu_{a}+\mu_{b}} w_{a} w_{b}\right)_{\infty}} \prod_{j=1}^{\ell(\mu)} \frac{\left(q^{\mu_{m}} w_{j}^{2}\right)_{\infty}}{\left(w_{j}^{2}\right)_{\infty}} \frac{\mathcal{G}^{q}\left(w_{j}\right)}{\mathcal{G}^{q}\left(q^{\mu_{j}} w_{j}\right)} d w_{j},
\end{aligned}
$$

where the positively oriented contours enclose $a_{j}$ and no other singularity.

Proof 1. To extract the coefficient of $z^{r}$ in (85), we use the $q$-binomial theorem (12) on the left-hand side. On the right-hand side, we may transform the integration over $s_{i}$ into a discrete sum using Lemma 3.17 and collect the terms of degree $k$ in $z$. We obtain

$$
\frac{1}{(q ; q)_{r}} \mathbb{E}^{q}\left[q^{r \lambda_{n}}\right]=\sum_{k=0}^{\infty} \frac{1}{k !} \sum_{\substack{v_{1}, \ldots, v_{k} \geqslant 1 \\ \nu_{1}+\cdots+v_{k}=r}} \oint \frac{d w_{1}}{2 \mathbf{i} \pi} \cdots \oint \frac{d w_{k}}{2 \mathbf{i} \pi} \operatorname{det}\left[\frac{1}{w_{i} q^{v_{i}}-w_{j}}\right]_{i, j=1}^{k}
$$




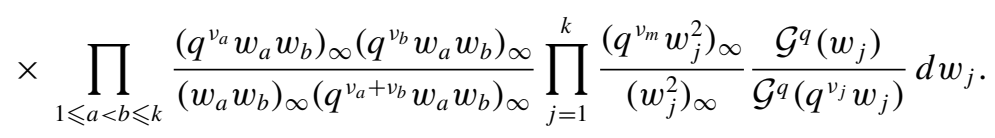

We may relabel $w_{j}$ 's since they are integrated on the same contour, or equivalently we may relabel $v_{j}$ 's. Hence, the sum over compositions $v$ can be rewritten as a sum over partitions $\mu$ (the factor $k ! /\left(m_{1} ! m_{2} ! \ldots\right)$ corresponds to the number of compositions corresponding to the same partition).

Proof 2. We present a second proof deriving Corollary 4.4 directly from Corollary 4.1. Although this proof is longer, it is useful to compare our present results with [BC14] and [BBC16], and a similar approach will be useful in Section 5. We first recall a useful result in the theory of Macdonald processes. It was first stated in [BC14] as Proposition 3.2.1, but we use a slightly more general version from [BCPS15b].

PROPOSITION 4.5 [BCPS15b, Proposition 7.4]. Let $\gamma_{1}, \ldots, \gamma_{k}$ be positively oriented closed contours and a function $F\left(z_{1}, \ldots, z_{k}\right)$ be such that we have the following:

- The contour $\gamma_{k}$ is a small circle around 1 , small enough so as to not contain $q$.

- For all $A<B, \gamma_{A}$ enclose $q \gamma_{B}$.

- For all $1 \leqslant j \leqslant k$, one can deform $\gamma_{j}$ to $\gamma_{k}$ in such a way that the function

$$
\frac{F\left(z_{1}, \ldots, z_{k}\right)}{z_{1} \ldots z_{k}} \prod_{i<j}\left(z_{i}-z_{j}\right)
$$

is analytic in $z_{j}$ (when all variables lie on their respective contours) in a neighborhood of the area swept by the deformation.

Then we have

$$
\begin{aligned}
& \oint \frac{d z_{1}}{2 \mathbf{i} \pi} \cdots \oint \frac{d z_{k}}{2 \mathbf{i} \pi} \prod_{A<B} \frac{z_{A}-z_{B}}{z_{A}-q z_{B}} \frac{F\left(z_{1}, \ldots, z_{k}\right)}{z_{1} \ldots z_{k}} \\
& =\sum_{\lambda \vdash k} \oint \frac{d w_{1}}{2 \mathbf{i} \pi} \cdots \oint \frac{d w_{\ell(\lambda)}}{2 \mathbf{i} \pi} d \mu_{\lambda}(\vec{w}) E^{q}(\vec{w} \circ \lambda),
\end{aligned}
$$

where

$$
\vec{w} \circ \lambda=\left(w_{1}, q w_{1}, \ldots, q^{\lambda_{1}-1} w_{1}, w_{2}, \ldots, q^{\lambda_{2}-1} w_{2}, \ldots, q^{\lambda_{\ell(\lambda)}-1} w_{\ell(\lambda)}\right),
$$




$$
E^{q}(\vec{z})=\sum_{\sigma \in \mathcal{S}_{k}} \sigma\left(\prod_{A<B} \frac{z_{A}-q z_{B}}{z_{A}-z_{B}} F\left(z_{1}, \ldots, z_{k}\right)\right)
$$

and

$$
d \mu_{\lambda}(\vec{w})=\frac{(q-1)^{k} q^{-\frac{k(k-1)}{2}}}{m_{1} ! m_{2} ! \ldots} \operatorname{det}\left[\frac{1}{w_{i} q^{\lambda_{i}}-w_{j}}\right]_{i, j=1}^{\ell(\lambda)} \prod_{j=1}^{\ell(\lambda)} d w_{j}
$$

We may apply Proposition 4.5 to (83) with the benign modification that the contour $\gamma_{k}$ is around $a_{i}$ instead of around 1 . Note that the factor

$$
\prod_{1 \leqslant a<b \leqslant k} \frac{1-q z_{a} z_{b}}{1-z_{a} z_{b}} \prod_{i=1}^{k} \frac{1}{1-z_{i}^{2}}
$$

is symmetric and can be taken outside of the sum over permutations in $E^{q}$. The evaluation into $\vec{w} \circ \lambda$ is given by the following.

LEMMA 4.6. For a partition $\lambda \vdash k$ with $m=\ell(\lambda)$, if $\vec{z}=\vec{w} \circ \lambda$ (the notation was introduced in (87)) then we have

$$
\begin{aligned}
& \prod_{1 \leqslant a<b \leqslant k} \frac{1-q z_{a} z_{b}}{1-z_{a} z_{b}} \prod_{i=1}^{k} \frac{1}{1-z_{i}^{2}} \\
& =\prod_{1 \leqslant a<b \leqslant m} \frac{\left(q^{\lambda_{a}} w_{a} w_{b}\right)_{\infty}\left(q^{\lambda_{b}} w_{a} w_{b}\right)_{\infty}}{\left(w_{a} w_{b}\right)_{\infty}\left(q^{\lambda_{a}+\lambda_{b}} w_{a} w_{b}\right)_{\infty}} \prod_{j=1}^{m} \frac{\left(q^{\lambda_{j}} w_{j}^{2}\right)_{\infty}}{\left(w_{j}^{2}\right)_{\infty}} .
\end{aligned}
$$

Proof. For $\vec{z}=\vec{w} \circ \lambda$, the left-hand side of (88) equals

$$
\begin{aligned}
(88)= & \prod_{1 \leqslant i<j \leqslant m} \prod_{r=0}^{\lambda_{i}-1} \prod_{s=0}^{\lambda_{j}-1} \frac{1-q^{1+r+s} w_{i} w_{j}}{1-q^{r+s} w_{i} w_{j}} \\
& \times \prod_{i=1}^{m} \prod_{0 \leqslant r<s \leqslant \lambda_{i}-1} \frac{1-q^{1+r+s} w_{i}^{2}}{1-q^{r+s} w_{i}^{2}} \prod_{t=0}^{\lambda_{i}-1} \frac{1}{1-q^{2 t} w_{i}^{2}} \\
= & \prod_{1 \leqslant i<j \leqslant m} \prod_{r=0}^{\lambda_{i}-1} \frac{1-q^{r+\lambda_{j}} w_{i} w_{j}}{1-q^{r} w_{i} w_{j}} \prod_{i=1}^{m} \prod_{r=0}^{\lambda_{i}-1} \frac{1-q^{r+\lambda_{i}} w_{i}^{2}}{1-q^{2 r+1} w_{i}^{2}} \frac{1}{1-q^{2 r} w_{i}^{2}} \\
= & \prod_{1 \leqslant i<j \leqslant m} \frac{\left(q^{\lambda_{j}} w_{i} w_{j}\right)_{\infty} /\left(q^{\lambda_{i}+\lambda_{j}} w_{i} w_{j}\right)_{\infty}}{\left(w_{i} w_{j}\right)_{\infty} /\left(q^{\lambda_{i}} w_{i} w_{j}\right)_{\infty}} \prod_{i=1}^{\lambda_{i}-1} \frac{1}{r=0} \frac{1}{1-q^{r} w_{i}^{2}}=\text { R.H.S. (88). }
\end{aligned}
$$


$E^{q}$ can then be computed using the symmetrization identity [Mac95, VII, (1.4)]

$$
\sum_{\sigma \in \mathcal{S}_{k}} \sigma\left(\prod_{1 \leqslant i<j \leqslant k} \frac{u_{i}-q u_{j}}{u_{i}-u_{j}}\right)=\frac{(q ; q)_{k}}{(1-q)^{k}} .
$$

Thus, Proposition 4.5 applied to Corollary 4.1 using Lemma 4.6 yields Corollary 4.4.

Now we provide formulas characterizing the distribution of $q^{-\lambda_{1}}$.

COROllary 4.7. Let $z \in \mathbb{C} \backslash \mathbb{R}_{>0}$. Assume the following:

(i) The parameters $a_{1}, \ldots, a_{n} \in(0,1)$ are chosen such that for all $i, j$, $\max \left\{a_{i}\right\}<a_{i} / a_{j}$ and $q a_{i} / a_{j}<1$.

(ii) $R \in(0,1), q^{R}>\max \left\{a_{i}\right\}$, and for all $i, j q^{R}<a_{i} / a_{j}$.

(iii) The $\alpha_{i}$ 's are chosen so that $q^{R}>\max \left\{a_{i}\right\} \max \left\{\alpha_{j}\right\}$.

Then, under the $q$-Whittaker measure $\mathbb{P}_{\left(a_{1}, \ldots, a_{n}\right),\left(\left(\alpha_{1}, \ldots, \alpha_{\ell}\right), \gamma\right)}^{q}$, we have

$$
\begin{aligned}
\mathbb{E}^{q}\left[\frac{1}{\left(z q^{-\lambda_{1}}\right)_{\infty}}\right] \\
=\sum_{k=0}^{n} \frac{1}{k !} \int_{\mathcal{D}_{R}} \frac{d s_{1}}{2 \mathbf{i} \pi} \cdots \int_{\mathcal{D}_{R}} \frac{d s_{k}}{2 \mathbf{i} \pi} \oint \frac{d w_{1}}{2 \mathbf{i} \pi} \cdots \oint \frac{d w_{k}}{2 \mathbf{i} \pi} \\
\quad \times \prod_{1 \leqslant i<j \leqslant k} \frac{\left(q^{s_{j}} w_{i}-q^{s_{i}} w_{j}\right)\left(w_{j}-w_{i}\right)\left(q^{-s_{i}} w_{i} w_{j}\right)_{\infty}\left(q^{-s_{j}} w_{i} w_{j}\right)_{\infty}}{\left(q^{s_{j}} w_{i}-w_{j}\right)\left(q^{s_{i}} w_{j}-w_{i}\right)\left(q^{-s_{i}-s_{j}} w_{i} w_{j}\right)_{\infty}\left(w_{i} w_{j}\right)_{\infty}} \\
\quad \times \prod_{i=1}^{k}\left[\Gamma\left(-s_{i}\right) \Gamma\left(1+s_{i}\right) \frac{\overline{\mathcal{G}}^{q}\left(w_{i}\right)}{\overline{\mathcal{G}}^{q}\left(q^{-s_{i}} w_{i}\right)} \frac{\left(q^{-s_{i}} w_{i}^{2}\right)_{\infty}(-z)^{s_{i}}}{\left(w_{i}^{2}\right)_{\infty}\left(1-q^{s_{i}}\right) w_{i}}\right],
\end{aligned}
$$

where the $w$ contours are small positively oriented circles enclosing $a_{i}$ and no other singularity, and $\mathcal{D}_{R}=R+\mathbf{i} \mathbb{R}$ oriented upwards, as before.

Proof. This is the $t=0$ degeneration of Theorem 3.20.

REMARK 4.8. Note that in light of similarities with $q$-Laplace transform formulas for the full-space $q$-Whittaker process-see in particular [BCFV15, Theorem 3.3] - it is conceivable that (90) holds as well if the contours for the $w_{i}$ variables contain not only the singularities $a_{i}$ but also $q^{j} a_{i}$ for all $1 \leqslant j \leqslant k$ for an arbitrary integer $k$. (In [BCFV15, Theorem 3.3], the contour encloses 
all the singularities $1 /\left(q^{j} a_{i}\right)$, but the formula would be valid as well if the contour would contain only the singularities $1 /\left(q^{j} a_{i}\right)$ for $1 \leqslant j \leqslant k$, where $k$ is an arbitrary positive integer.) Transforming the contours in such a way may be convenient for later asymptotic analysis of the formula, so as to work with infinite contours in Section 6. Presently, it is not clear how to justify such a contour deformation.

Both sides of (90) are analytic in $z \in \mathbb{C} \backslash \mathbb{R}_{\geqslant 0}$ but not analytic at $z=0$, so one cannot extract coefficients as in the proof of Corollary 4.4. Nevertheless, we have the following:

COROLlaRY 4.9. For all $r \in \mathbb{Z}_{>0}$ such that $q^{r}>\left(\max \left\{a_{j}\right\}\right)^{2}$ and $q^{r}>$ $\max \left\{\alpha_{j}\right\} \max \left\{a_{j}\right\}$, under the $q$-Whittaker measure $\mathbb{P}_{\left(a_{1}, \ldots, a_{n}\right),\left(\left(\alpha_{1}, \ldots, \alpha_{\ell}\right), \gamma\right)}^{q}$,

$$
\begin{aligned}
& \mathbb{E}^{q}\left[q^{-r \lambda_{1}}\right] \\
& =(q ; q)_{r} \sum_{\substack{\mu \vdash r \\
\mu=1^{m} 2^{m_{2}} \ldots}} \frac{1}{m_{1} ! m_{2} ! \ldots} \oint \frac{d w_{1}}{2 \mathbf{i} \pi} \cdots \oint \frac{d w_{\ell(\mu)}}{2 \mathbf{i} \pi} \\
& \quad \times \operatorname{det}\left[\frac{1}{q^{\mu_{i}} w_{i}^{-1}-w_{j}^{-1}}\right]_{i, j=1}^{\ell(\mu)} \prod_{1 \leqslant a<b \leqslant \ell(\mu)} \frac{\left(q^{-\mu_{a}} w_{a} w_{b}\right)_{\infty}\left(q^{-\mu_{b}} w_{a} w_{b}\right)_{\infty}}{\left(w_{a} w_{b}\right)_{\infty}\left(q^{-\mu_{a}-\mu_{b}} w_{a} w_{b}\right)_{\infty}} \\
& \quad \times \prod_{j=1}^{\ell(\mu)} \frac{\left(q^{-\mu_{j}} w_{j}^{2}\right)_{\infty}}{\left(w_{j}^{2}\right)_{\infty}} \frac{\overline{\mathcal{G}}^{q}\left(w_{j}\right)}{\overline{\mathcal{G}}^{q}\left(q^{-\mu_{j}} w_{j}\right)} \frac{d w_{j}}{w_{j}^{2}}
\end{aligned}
$$

where the positively oriented contours enclose $a_{j}$ and no other singularity.

Proof. Let $D_{q}$ be the $q$-derivative operator defined by

$$
D_{q} f(z)=\frac{f(q z)-f(z)}{q z-z} .
$$

We have

$$
D_{q}\left(\frac{1}{\left(z q^{-\lambda_{1}}\right)_{\infty}}\right)=\frac{q^{-\lambda_{1}}}{(1-q)\left(z q^{-\lambda_{1}}\right)_{\infty}} .
$$

For $z \in \mathbb{C} \backslash \mathbb{R}_{\geqslant 0}$, we can apply $D_{q}$ to the left-hand side of (90), and the $q$ derivative clearly commutes with the expectation, so that if we iterate the procedure, we get

$$
\left(D_{q}\right)^{r} \mathbb{E}^{q}\left[\frac{1}{\left(z q^{-\lambda_{1}}\right)_{\infty}}\right]=\mathbb{E}^{q}\left[\frac{q^{-r \lambda_{1}}}{(1-q)^{r}\left(z q^{-\lambda_{1}}\right)_{\infty}}\right] .
$$


If $\mathbb{E}^{q}\left[q^{-r \lambda_{1}}\right]$ is finite, we may let $z$ tend to 0 in (92) to obtain

$$
\mathbb{E}^{q}\left[q^{-r \lambda_{1}}\right]=(1-q)^{r} \lim _{z \rightarrow 0}\left\{\left(D_{q}\right)^{r} \mathbb{E}^{q}\left[\frac{1}{\left(z q^{-\lambda_{1}}\right)_{\infty}}\right]\right\} .
$$

Now we can apply $D_{q}$ to the right-hand side of (90). If $q^{r}>\left(\max \left\{a_{j}\right\}\right)^{2}$ and $q^{r}>\max \left\{\alpha_{j}\right\} \max \left\{a_{j}\right\}$, we may shift the contour $\mathcal{D}_{R}$ to $\mathcal{D}_{R+r}$ and we will encounter only the poles at $s=1, \ldots, r$ during the contour deformation. The sum of the residues is a polynomial in the variable $z$. We may rewrite the application of $\left(D_{q}\right)^{r}$ to (90) as the sum of $\left(D_{q}\right)^{r}$ applied to this polynomial and $\left(D_{q}\right)^{r}$ applied to the integral remainder. We have $D_{q}(-z)^{s}=\frac{1-q^{s}}{1-q}(-z)^{s-1}$, so after applying $\left(D_{q}\right)^{r}$ to the integrand in (90), it gets multiplied by

$$
\frac{1-q^{s_{1}+\cdots+s_{k}}}{1-q} \frac{1-q^{s_{1}+\cdots+s_{k}-1}}{1-q} \ldots \frac{1-q^{s_{1}+\cdots+s_{k}-r+1}}{1-q}(-z)^{-r} .
$$

Hence, after shifting the contours and applying $\left(D_{q}\right)^{r}$, the integral term goes to zero as $z \rightarrow 0$ because the factor $z^{-r} \prod_{i=1}^{k}(-z)^{s_{i}}$ with $\mathfrak{R e}\left[s_{i}\right]=R+r$ goes to zero. For a polynomial $P(X)=\sum_{i=1}^{N} c_{i} X^{i}$,

$$
\lim _{z \rightarrow 0}\left\{\left(D_{q}\right)^{r} P(z)\right\}=c_{r} \prod_{i=1}^{r} \frac{1-q^{i}}{1-q} .
$$

In our context, $c_{r}$ corresponds to the contribution of residues at $s_{1}=\mu_{1}, \ldots$, $s_{k}=\mu_{k}$ for some $\mu_{1}, \ldots, \mu_{k} \in \mathbb{Z}_{>0}$ such that $\mu_{1}+\cdots+\mu_{k}=r$. These residues are easy to compute and they can be written as in (91).

REMARK 4.10. Corollary 4.9 could also be proved from (84) by shrinking the nested contours to a small contour around $a_{i}$ (that is, applying Proposition 4.5) as in the proof of Corollary 4.4 .

REMARK 4.11. Regarding the distribution of $q^{\lambda_{n}}$, the moment formula in Corollary 4.4 and the Laplace transform formula from Corollary 4.3 are essentially equivalent (for sufficiently small parameters $a_{1}, \ldots, a_{n}$ ). Indeed, we have deduced the moment formulas from the Laplace transform but one can go backwards summing the $q$-moment generating series.

However, turning to the distribution of $q^{-\lambda_{1}}$, the moment formula from Corollary 4.9 can be deduced from the Laplace transform formula from Corollary 4.7, but one cannot go backwards (otherwise, this would have been a much easier route to prove Corollary 4.7). Let us see why. Denoting the 
left-hand side in (90) by $L(z)$ and the right-hand side by $R(z)$, Corollary 4.9 implies that for all $n \geqslant 0$,

$$
\lim _{z \rightarrow 0}\left(D_{q}\right)^{n} R(z)=\lim _{z \rightarrow 0}\left(D_{q}\right)^{n} L(z) .
$$

This is not sufficient to deduce Corollary 4.7. Indeed, as for the usual differential operator, a function $f(z)$ is in general not determined in a neighborhood around 0 by the knowledge of $\lim _{z \rightarrow 0}\left(D_{q}\right)^{n} f(z)$ for all $n \in \mathbb{Z}_{\geqslant 0}$, unless it is analytic at 0 . As a counterexample, one may consider

$$
f(z)=\sum_{k=0}^{\infty} \frac{\theta^{k}(\theta)_{\infty}}{\left(z q^{-k}\right)_{\infty}(q ; q)_{k}} \quad \text { and } \quad g(z)=\sum_{k=0}^{\infty} \frac{z^{k}(z)_{\infty}}{\left(\theta q^{-k}\right)_{\infty}(q ; q)_{k}}
$$

and check that

$$
\lim _{z \rightarrow 0}\left(D_{q}\right)^{n} f(z)=\frac{1}{\left(\theta q^{-n}\right)_{\infty}(1-q)^{n}}=\lim _{z \rightarrow 0}\left(D_{q}\right)^{n} g(z) .
$$

In the above counterexample, $f(z)$ should be thought of as the correct expression for the Laplace transform while $g(z)$ is the wrong expression that one would obtain by summing moment formulas (or applying the operator $\overline{\mathbf{N}}^{z}$ instead of $\mathbf{M}^{z}$ ). (We have that $f(z)=\mathbb{E}^{q}\left[\frac{1}{\left(z q^{-\lambda_{1}}\right)_{\infty}}\right]$, where $\lambda_{1}$ is distributed according to the half-space $q$-Whittaker measure with specializations $\rho=\left(a_{1}\right)$ and $\rho^{\swarrow}=\left(\alpha_{1}\right)$ with $a_{1} \alpha_{1}=\theta$.)

4.2. Matveev-Petrov RSK-type dynamics. Section 2.4 introduced a general scheme through which one can grow half-space Macdonald processes using two types of operators $\mathcal{U}^{\llcorner}$and $\mathcal{U}^{L}$. The main condition required of these operators was that they satisfied the defining relations (36) and (37). MatveevPetrov [MP17] provide four different choices for the bulk operator $\mathcal{U}^{\llcorner}$that solve (36), provided the partitions satisfy certain interlacing conditions and the specializations are chosen appropriately. The description of these operators is quite involved and overall unnecessary for our purposes. Instead, we will recall the relevant properties of these operators one by one.

We will use only two of the bulk $\mathcal{U}^{\llcorner}$operators introduced in [MP17], and denote them as $\mathcal{U}_{\text {row }}^{\llcorner}[\alpha]$ and $\mathcal{U}_{\text {col }}^{\llcorner}[\alpha]$, where $\alpha$ is a generic positive real number. These transition operators correspond, in the Schur process degeneration, to dynamics on Gelfand-Tsetlin patterns induced by the row or column RSK insertion, respectively (see [Knu70] or [MP17, Section 4.3] and references therein). 
REMARK 4.12. It would be tempting to consider the two other possibilities $\mathcal{U}_{\text {row }}^{\llcorner}[\hat{\beta}], \mathcal{U}_{\text {col }}^{\llcorner}[\hat{\beta}]$, which are dual analogues. However, this is not presently accessible due to our choice of $\mathcal{U}^{L}$, which enforces the use of the same set of specializations on horizontal and vertical edges.

$\mathcal{U}_{\text {row }}^{\llcorner}[\alpha]$ and $\mathcal{U}_{\text {col }}^{\llcorner}[\alpha]$ act from the subspace of $\kappa \otimes \mu \otimes v \in \mathbb{Y}_{k} \otimes \mathbb{Y}_{k} \otimes \mathbb{Y}_{k+1}$ such that $\mu \prec v$ and $\mu \prec \kappa$ to the subspace of $\kappa \otimes \pi \otimes v \in \mathbb{Y}_{k} \otimes \mathbb{Y}_{k+1} \otimes \mathbb{Y}_{k+1}$ such that $\kappa \prec \pi$ and $v \prec \pi$. For such partitions $\kappa, \mu, v, \pi$, we will use the notation $\mathcal{U}^{\llcorner}(\pi \mid \kappa, \mu, \nu)$ as in Section 2.4. For a partition $\lambda \in \mathbb{Y}_{k}$ and $1 \leqslant j \leqslant k$, let $\overrightarrow{\operatorname{Proj}}_{j}(\lambda)=\left(\lambda_{1}, \ldots, \lambda_{j}\right)$ be the projection of $\lambda$ onto its first $j$ parts (now a partition in $\mathbb{Y}_{j}$ ). (The orientation of arrows in $\overrightarrow{\text { Proj }}$ and $\overleftarrow{\text { Proj }}$ is chosen to be consistent with Figure 4 and interlacing arrays in Section 6.4.) Similarly, let $\overleftarrow{\operatorname{Proj}}_{j}(\lambda)=\left(\lambda_{k-j+1}, \ldots, \lambda_{k}\right)$ be the projection of $\lambda$ onto its last $j$ parts. The main fact we use from [MP17] is that $\mathcal{U}_{\text {row }}^{\llcorner}[\alpha]$ and $\mathcal{U}_{\text {col }}^{\llcorner}[\alpha]$ satisfy the defining relation (36), and their projections under $\overrightarrow{\operatorname{Proj}}_{j}$ and $\overleftrightarrow{\operatorname{Proj}}_{j}$ are marginally Markov.

We will continue to use the boundary transition operator $\mathcal{U}^{L}$ of push-block type defined in Section 2.4.2, although other choices might be interesting to study and could lead to different dynamics. Recall that in the setting of Section 2.4, the operators $\mathcal{U}^{\llcorner}$and $\mathcal{U}^{L}$ depend on specializations $\rho_{\text {。 }}$ and $\rho_{i}$ for $i \geqslant 1$.

4.2.1. Boundary transition operator. We recall that for $k \geqslant 0$ and partitions $\mu \in \mathbb{Y}_{k}, \kappa, \pi \in \mathbb{Y}_{k+1}$,

$$
\mathcal{U}_{k, k}^{L}(\pi \mid \kappa, \mu)=\mathcal{U}_{k, k}^{L}(\pi \mid \kappa)=\frac{P_{\pi / \kappa}\left(\rho_{k+1}\right) \mathcal{E}_{\pi}\left(\rho_{\circ}\right)}{\mathcal{E}_{\kappa}\left(\rho_{k+1}, \rho_{\circ}\right) \Pi\left(\rho_{k+1}, \rho_{\circ}\right) \Phi\left(\rho_{k+1}\right)} .
$$

The right-hand side above can be computed more explicitly when the specializations are simply the evaluation into single variables.

LeMmA 4.13. For $a, b \in \mathbb{C}$, we have

$$
\mathcal{E}_{\mu}(a) P_{\mu / \lambda}(b)=a^{\sum_{i} \mu_{2 i-1}-\mu_{2 i}} b^{\sum_{i} \mu_{i}-\lambda_{i}} \prod_{i=1}^{\ell(\mu)} \frac{\left(q^{\mu_{i}-\lambda_{i}+1} ; q\right)_{\infty}\left(q^{\lambda_{i}-\mu_{i+1}+1} ; q\right)_{\infty}}{(q ; q)_{\infty}^{2}} .
$$

Proof. Using the combinatorial formula (20), $Q_{\mu / \eta}(u)=u^{|\mu|-|\eta|} \varphi_{\mu / \eta} \mathbf{1}_{\eta<\mu}$, where

$$
\varphi_{\mu / \eta}=\prod_{i=1}^{\ell(\mu)} \frac{\left(q^{\mu_{i}-\eta_{i}+1} ; q\right)_{\infty}\left(q^{\eta_{i}-\mu_{i+1}+1} ; q\right)_{\infty}}{(q ; q)_{\infty}\left(q^{\eta_{i}-\eta_{i+1}+1} ; q\right)_{\infty}} .
$$

We would like to evaluate $\mathcal{E}_{\mu}(u)$. Since $u$ is a single usual specialization, $Q_{\mu / \eta}(u)$ is only nonzero when $\mu / \eta$ is a horizontal strip. Among those $\eta$, there is 
only one that has $\eta^{\prime}$ even, and it is given by $\eta^{*}=\left(\mu_{2}, \mu_{2}, \mu_{4}, \mu_{4}, \ldots\right)$. For that $\eta^{*}$,

$$
\varphi_{\mu / \eta^{*}}=\prod_{i=1}^{\ell(\mu)} \frac{\left(q^{\mu_{i}-\mu_{i+1}+1} ; q\right)_{\infty}}{(q ; q)_{\infty}} \prod_{j=1}^{\lfloor\ell(\mu) / 2\rfloor} \frac{(q ; q)_{\infty}}{\left(q^{\mu_{2 j}-\mu_{2 j+2}+1} ; q\right)_{\infty}} .
$$

We also readily see that for $\eta^{*}$, the only boxes for which $b_{\eta^{*}}(\square)$ does not equal 1 are those that have leg length 0 (otherwise, the factor of $t^{\operatorname{leg}}$ in the denominator is zero). Thus, we find

$$
b_{\eta^{*}}^{\mathrm{el}}=\prod_{j=1}^{\lfloor\ell(\mu) / 2\rfloor} \frac{\left(q^{\mu_{2 j}-\mu_{2 j+2}+1} ; q\right)_{\infty}}{(q ; q)_{\infty}}
$$

so that

$$
\mathcal{E}_{\mu}(u)=b_{\eta^{*}}^{\mathrm{el}} u^{\sum_{i} \mu_{2 i-1}-\mu_{2 i}} \varphi_{\mu / \eta^{*}}=u^{\sum_{i} \mu_{2 i-1}-\mu_{2 i}} \prod_{i=1}^{\ell(\mu)} \frac{\left(q^{\mu_{i}-\mu_{i+1}+1} ; q\right)_{\infty}}{(q ; q)_{\infty}} .
$$

By the combinatorial formula (19),

$$
P_{\mu / \lambda}(u)=u^{|\mu|-|\lambda|} \psi_{\mu / \lambda},
$$

where

$$
\psi_{\mu / \lambda}=\prod_{i=1}^{\ell(\lambda)} \frac{\left(q^{\mu_{i}-\lambda_{i}+1} ; q\right)_{\infty}\left(q^{\lambda_{i}-\mu_{i+1}+1} ; q\right)_{\infty}}{(q ; q)_{\infty}\left(q^{\mu_{i}-\mu_{i+1}+1} ; q\right)_{\infty}} .
$$

Putting this all together and noting that $\ell(\lambda)+1=\ell(\mu)$, we arrive at (93).

DEFINITION 4.14. A q-geometric random variable with parameter $\theta \in(0,1)$, denoted by $q \operatorname{Geom}(\theta)$, has distribution

$$
\mathbb{P}(X=k)=\frac{\theta^{k}}{(q ; q)_{k}}(\theta ; q)_{\infty}, \quad k \in \mathbb{Z}_{\geqslant 0}
$$

LEMMA 4.15. Assume that $\rho_{\circ}$ and $\rho_{i}$ are specializations into single variables $a_{\circ}$ and $a_{i}$, respectively, for all $i \geqslant 1$.

(1) For any partitions $\mu \prec \kappa \in \mathbb{Y}_{k}$, the probability kernel $\mathcal{U}_{k, k}^{L}(\pi \mid \kappa, \mu)$ is supported on $\pi \in \mathbb{Y}_{k+1}$. Moreover, for any $j \leqslant k$, the push-forward of $\mathcal{U}_{k, k}^{L}(\pi \mid \kappa, \mu)$ with respect to $\overrightarrow{\mathrm{Proj}}_{j}$ (the marginal distribution of $\pi$ corresponding to its first $j$ parts) depends only on $\overrightarrow{\operatorname{Proj}}_{j}(\kappa)$. 
(2) The projection of the dynamics $\mathcal{U}_{k, k}^{\perp}(\pi \mid \kappa, \mu)$ to the first part is given by

$$
\pi_{1}=\kappa_{1}+\mathrm{qGeom}\left(a_{\circ} a_{k+1}\right) .
$$

Proof. For fixed $\mu \prec \kappa \in \mathbb{Y}_{k}, \mathcal{U}^{L}(\pi \mid \kappa, \mu)$ is proportional to

$$
P_{\pi / \kappa}\left(a_{k+1}\right) \mathcal{E}_{\pi}\left(a_{\circ}\right)=a_{\circ}^{\sum_{i} \pi_{2 i-1}-\pi_{2 i}} a_{k+1}^{\sum_{i} \pi_{i}-\kappa_{i}} \prod_{i=1}^{\ell(\pi)} \frac{1}{(q ; q)_{\pi_{i}-\kappa_{i}}(q ; q)_{\kappa_{i}-\pi_{i+1}}} \mathbf{1}_{\kappa \prec \pi},
$$

as follows from Lemma 4.13. By summing over $\pi_{j+1}, \pi_{j+2}, \ldots$, we see that the distribution of $\overrightarrow{\operatorname{Proj}}_{j}(\pi)$ depends only on $\overrightarrow{\operatorname{Proj}}_{j}(\kappa)$. In particular, summing over $\pi_{2}, \ldots, \pi_{k+1}$, we find that the weight of $\pi_{1}$ is proportional to

$$
\frac{\left(a_{\circ} a_{k+1}\right)^{\pi_{1}}}{(q ; q)_{\pi_{1}-\kappa_{1}}} \mathbf{1}_{\pi_{1} \geqslant \kappa_{1}}
$$

which shows that $\pi_{1}-\kappa_{1}$ has the qGeom $\left(a_{\circ} a_{k+1}\right)$ distribution.

DEFINITION 4.16. We introduce the q-inverse Gaussian distribution (see Lemma 6.28 for a justification of the name) with parameters $m \in \mathbb{Z}_{\geqslant 0} \cup\{+\infty\}$ and $\theta \in(0,1)$. A $q$-inverse Gaussian random variable $X$ is such that

$$
\mathbb{P}(X=k)=\theta^{k} \frac{(q ; q)_{m}}{(q ; q)_{k}(q ; q)_{m-k}} \frac{1}{Z_{m}(\theta)}, \quad k \in\{0, \ldots, m\},
$$

where $Z_{m}(\theta)$ is the $m$ th Rogers-Szegő polynomial

$$
Z_{m}(\theta)=\sum_{k=0}^{m} \theta^{k} \frac{(q ; q)_{m}}{(q ; q)_{k}(q ; q)_{m-k}}
$$

When $m=\infty$, the $q$-inverse Gaussian distribution degenerates to the $q$ geometric one.

LEMMA 4.17. Assume that $\rho_{\circ}$ and $\rho_{i}$ are specializations into single variables $a_{\circ}$ and $a_{i}$, respectively, for all $i \geqslant 1$.

(1) For any $j \leqslant k$, and partitions $\mu \prec \kappa \in \mathbb{Y}_{k}$, the push-forward of $\mathcal{U}_{k, k}^{L}(\pi \mid \kappa$, $\mu)$ with respect to $\overleftarrow{\operatorname{Proj}}_{j}$ (last j parts of the partitions) depends only on $\overleftarrow{\operatorname{Proj}}_{j}(\kappa)$

(2) The projection of the dynamics $\mathcal{U}_{k, k}^{L}(\pi \mid \kappa, \mu)$ on the last part is such that $\pi_{k+1}$ is a $q$-inverse Gaussian random variable with parameters $\kappa_{k}$ and $a_{\circ}^{(-1)^{k}} a_{k+1}$. 


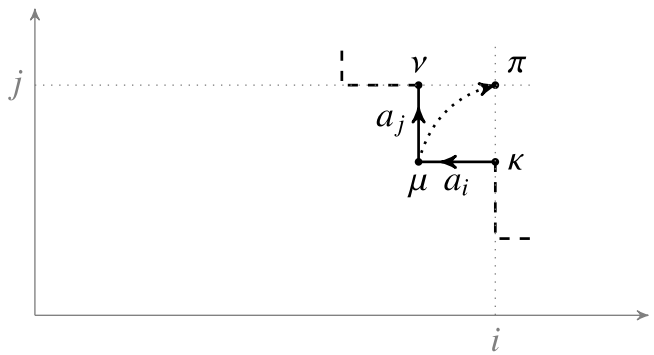

Figure 9. Action of the transition operators $\mathcal{U}_{\text {row }}^{\llcorner}(\pi \mid \kappa, \mu, \nu)$ and $\mathcal{U}_{\text {col }}^{\llcorner}(\pi \mid \kappa, \mu, v)$ on a corner formed by partitions $\kappa, \mu, v$ at positions $(i, j-1),(i-1, j-1)$, $(i-1, j)$. The dashed part of the path has no influence on the distribution of $\pi$.

Proof. One proves (1) similarly to Lemma 4.15. Summing over $\pi_{1}, \ldots, \pi_{k}$ for a fixed $\kappa \in \mathbb{Y}_{k}$, the distribution of $\pi_{k+1}$ is proportional to

$$
\frac{a_{\circ}^{(-1)^{k} \pi_{k+1}} a_{k+1}^{\pi_{k+1}}}{(q ; q)_{\pi_{k+1}}(q ; q)_{\kappa_{k}-\pi_{k+1}}},
$$

which proves (2).

4.2.2. Bulk dynamics based on RSK row insertion. For $j \geqslant 1$, consider four partitions $\kappa, \mu \in \mathbb{Y}_{j-1}$ and $\pi, v \in \mathbb{Y}_{j}$ such that

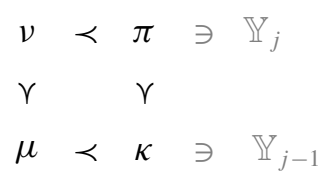

Assume that these partitions appear on a section of a path $\omega$ as in Figure 9 so that a specialization $\rho_{i}$ lies on the horizontal edge and $\rho_{j}$ lies on the vertical edge. Assume that $\rho_{i}$ and $\rho_{j}$ are specializations into single variables $a_{i}$ and $a_{j}$. Then, [MP17] defines an operator that we will call $\mathcal{U}_{\text {row }}^{\llcorner}(\pi \mid \kappa, \mu, v)$, which satisfies the relation in (36). We collect the following properties of $\mathcal{U}_{\text {row }}^{\llcorner}$from [MP17], wherein $\mathcal{U}_{\text {row }}^{\llcorner}(\pi \mid \kappa, \mu, \nu)$ is denoted by $\mathcal{U}_{j}(\nu \rightarrow \pi \mid \mu \rightarrow \kappa)$ when the sequence $\left(\mathcal{U}_{j}\right)$ defines the multivariate dynamics $\mathcal{Q}_{\text {row }}^{q}[\alpha]$; cf. [MP17, Section 6.2].

PROPOSITION 4.18. With the above notation, we have the following:

(1) $\mathcal{U}_{\text {row }}^{\llcorner}(\pi \mid \kappa, \mu, \nu)$ is supported on $\pi$ such that $\nu \prec \pi$ and $\kappa \prec \pi$ [MP17, Lemma 6.2]. 
(2) $\mathcal{U}_{\text {row }}^{\llcorner}(\pi \mid \kappa, \mu, \nu)$ preserves the $q$-Whittaker process structure, in the sense of (36) [MP17, Theorem 6.4].

(3) The projection of the dynamics on the first $j$ parts of each partition is marginally Markov [MP17, Section 6.3].

Definition 4.19. For parameters $q, \xi, \eta \in \mathbb{R}_{>0}, y \in\{0,1, \ldots\} \cup\{+\infty\}$ and $s \in\{0, \ldots, y\}$, define the $q$-beta-binomial distribution, denoted as $\operatorname{qBetaBin}(q$, $\xi, \eta, y)$, by the weights

$$
\boldsymbol{\varphi}_{q, \xi, \eta}(s \mid y)=\xi^{s} \frac{(\eta / \xi ; q)_{s}(\xi ; q)_{y-s}}{(\eta ; q)_{y}} \frac{(q ; q)_{y}}{(q ; q)_{s}(q ; q)_{y-s}} .
$$

These weights were introduced in [GO09, Pov13]. There are several ranges of parameters for which the weights $\boldsymbol{\varphi}_{q, \xi, \eta}(s \mid y)$ define a probability distribution on $\{0, \ldots, y\}$. The simplest choice is $0 \leqslant q<1$ and $0 \leqslant \eta<\xi<1$, and the corresponding distribution is a natural $q$-analogue of the beta-binomial distribution [GO09]. Another possibility, considered in [MP17], is to use the weights $\boldsymbol{\varphi}_{q^{-1}, q^{a}, q^{a+b}}(s \mid y)$, where $a, b$ are nonnegative integers such that $y \leqslant a+b$. We may consider also the degeneration when $b$ goes to infinity and denote the corresponding weights $\boldsymbol{\varphi}_{q^{-1}, q^{a}, 0}(s \mid y)$. Note that $\boldsymbol{\varphi}_{q, \xi, \eta}(s \mid y)$ degenerates to the $q$ geometric distribution when $\eta=0$ and $y=+\infty$.

The law of the first part marginals under the dynamics $\mathcal{U}_{\text {row }}^{\llcorner}$can be described explicitly as follows.

LEMMA 4.20 [MP17, Section 6.3]. Under the transition operator $\mathcal{U}_{\text {row }}^{\llcorner}(\pi \mid \kappa, \mu$, v),

$$
\pi_{1}=v_{1}+V+W
$$

where $V$ is distributed as a q-geometric random variable with parameter $a_{i} a_{j}$ and $W$ is distributed according to

$$
\mathbb{P}(W=k)=\varphi_{q^{-1}, q^{\nu_{1}-\mu_{1}, 0}}\left(k \mid \kappa_{1}-\mu_{1}\right) ;
$$

$V$ and $W$ are independent.

4.2.3. Bulk dynamics based on RSK column insertion. We will also use another type of dynamics that we will denote $\mathcal{U}_{\text {col }}^{\llcorner}(\pi \mid \kappa, \mu, v)$, introduced in [MP17] wherein it is denoted by $\mathcal{U}_{j}(\nu \rightarrow \pi \mid \mu \rightarrow \kappa)$, when the sequence $\left(\mathcal{U}_{j}\right)$ defines the multivariate dynamics $\mathcal{Q}_{\text {col }}^{q}[\alpha]$; cf. [MP17, Section 6.4]. As for the dynamics $\mathcal{U}_{\text {row }}^{\llcorner}$, we will not provide the complete definition of $\mathcal{U}_{\text {col }}^{\llcorner}$since we do not need it, but only list the following properties proved in [MP17]. 
PROPOSITION 4.21. Under the same assumptions as in Section 4.2.2, we have the following:

(1) $\mathcal{U}_{\text {col }}^{\llcorner}(\pi \mid \kappa, \mu, v)$ is supported on $\pi$ such that $\nu \prec \pi$ and $\kappa \prec \pi$ [MP17, Lemma 6.6].

(2) $\mathcal{U}_{c o l}^{\llcorner}(\pi \mid \kappa, \mu, v)$ satisfies (36) [MP17, Theorem 6.10].

(3) The projection of the dynamics on the last $j$ parts of each partition is marginally Markov [MP17, Section 6.6].

The dynamics on the last part of each partition under $\mathcal{U}_{\text {col }}^{\llcorner}(\pi \mid \kappa, \mu, \nu)$ are explicit.

LEMMA 4.22 [MP17, Section 6.6]. Under the transition operator $\mathcal{U}_{\mathrm{col}}^{\llcorner}(\pi \mid \kappa, \mu$, v),

$$
\pi_{k+1}=v_{k+1}+W
$$

where

$$
\mathbb{P}(W=j)=\varphi_{q, a_{i} a_{j}, 0}\left(j \mid \mu_{k}-v_{k+1}\right) .
$$

The distribution $\boldsymbol{\varphi}_{q, \theta, 0}(\cdot \mid m)$ can be seen as a truncation of the $q$-geometric distribution. It is a different truncation than the $q$-inverse Gaussian distribution (see Definition 4.16).

4.3. New exactly solvable particle systems. The dynamics studied in Section 4.2 suggest the definition of new exactly solvable particle systems, which are 'half-space' variants of the Geometric $q$-PushTASEP (introduced in [MP17]) and the discrete Geometric $q$-TASEP (introduced in [BC15]). For both particle systems, we can use the moment formulas from Corollaries 4.1, 4.9 and 4.4 and the Laplace transform formulas from Corollaries 4.7 and 4.3. This provides moment and Laplace transform formulas for the random variable $q^{ \pm x_{n}(t)}$, where $x_{n}(t)$ will denote the position of an arbitrary particle at any time $t$ in the models that we define now.

We start with the particle system corresponding to the row insertion dynamics (Section 4.2.2).

DEFINITION 4.23. The Geometric $q$-PushTASEP with particle creation is a discrete-time Markov process on configurations of particles

$$
0=x_{0}(t)<x_{1}(t)<x_{2}(t)<\cdots<x_{t}(t)<\infty \text {. }
$$




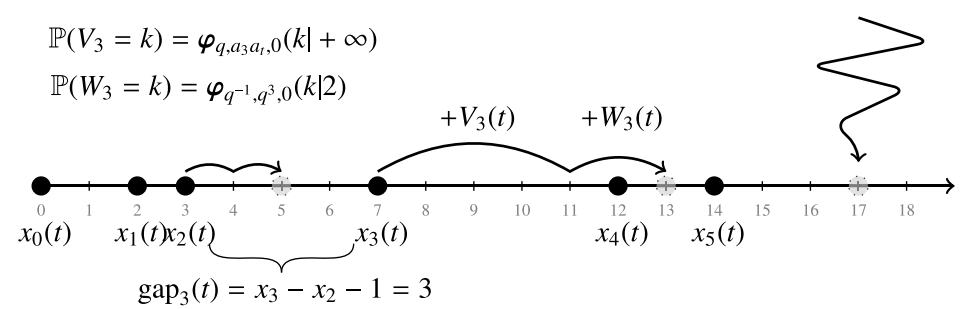

Figure 10. Configuration of particles in the Geometric $q$-PushTASEP with particle creation at time $t=5$. We illustrate possible jumps of the particles sitting at $x_{2}(t)$ and $x_{3}(t)$. Note that the particle sitting at $x_{4}(t)$ will necessarily jump to a position on the right of $x_{3}(t+1)$ (by the construction of $\left.W_{4}(t)\right)$. A new particle will be created at $x_{t+1}(t+1)(=17$ on the figure).

At time 0 , there is only one particle at $x_{0}(0)=0$. From time $t$ to $t+1$, particles' positions $x_{n}(t)$ for $n=1$ to $t$ are sequentially updated so that the particle at $x_{n}(t)$ jumps to its new location $x_{n}(t+1)=x_{n}(t)+V_{n}(t)+W_{n}(t)$, where $V_{n}(t)$ and $W_{n}(t)$ are independent, $V_{n}(t)$ is a $q$-geometric random variable with parameter $a_{n} a_{t}$ (see Definition 4.14) and $W_{n}(t)$ is distributed according to

$$
\mathbb{P}\left(W_{n}(t)=k\right)=\varphi_{q^{-1}, q^{\operatorname{gap}_{n}(t), 0}}\left(k \mid x_{n-1}(t+1)-x_{n-1}(t)\right) .
$$

where $\operatorname{gap}_{n}(t)=x_{n}(t)-x_{n-1}(t)-1$. Additionally, a new particle is created at location $x_{t+1}(t+1)=x_{t}(t+1)+V_{t+1}(t)+1$, where $V_{t+1}(t)$ is an independent $q$-geometric random variable with parameter $a_{\circ} a_{t}$ (see Figure 10). Note that the strict ordering of particle locations is preserved by the dynamics.

PROPOSITION 4.24. Let $\left(x_{n}(t)\right)_{1 \leqslant n \leqslant t}$ denote the positions of particles in the Geometric q-PushTASEP with particle creation (Definition 4.23). Let $\left(\lambda^{(t, n)}\right)_{1 \leqslant n \leqslant t}$ be a sequence of random partitions distributed according to the Markovian growth procedure described in Section 2.4, using transition operators $\mathcal{U}_{\text {row }}^{\llcorner}$and $\mathcal{U}^{L}$, and specializations $\rho_{\circ}$ and $\rho_{i}$ into single variables $a_{\circ}$ and $a_{i}$, respectively, for all $i \geqslant 1$. In particular, $\lambda^{(t, n)}$ is distributed according to the half-space $q$-Whittaker measure $\mathbb{P}_{\left(a_{1}, \ldots, a_{n}\right),\left(a_{0}, a_{n+1}, \ldots, a_{t}\right)}^{q}$. Then, for any admissible path $\omega \in \Omega$, we have

$$
\left(x_{n}(t)\right)_{(t, n) \in \omega} \stackrel{(d)}{=}\left(\lambda_{1}^{(t, n)}+n\right)_{(t, n) \in \omega} .
$$

Proof. The two families have the same dynamics, according to Lemmas 4.15 and 4.20. 
REMARK 4.25. The moments of $q^{-x_{n}(t)}$ are given by (84) in Corollary 4.1. By definition of the dynamics, $x_{n}(t)$ can be bounded by a sum of $q$-Geometric random variables with parameters $a_{\circ} a_{t^{\prime}}$ and $a_{n^{\prime}} a_{t^{\prime}}$ over some range of $t^{\prime}, n^{\prime}$. However, for $X \sim \mathrm{qGeom}(\theta), \mathbb{E}\left[q^{-k X}\right]$ is finite as long as $q^{k}>\theta$. This explains why the moments of $q^{-\lambda_{1}}$ in Corollary 4.1 can exist only when $q^{k}>\max \left\{a_{j}\right\}^{2}$ and $q^{k}>\max \left\{\alpha_{j}\right\} \max \left\{a_{j}\right\}$. It turns out that these conditions are sufficient for the existence of moments.

We introduce now the particle system corresponding to the column insertion dynamics (Section 4.2.3).

DEFINITION 4.26. The Geometric $q$-TASEP with activation is a discrete-time Markov process on infinite configurations of particles

$$
\cdots<x_{n}(t)<\cdots<x_{1}(t)<x_{0}(t) \equiv+\infty .
$$

At time $0, x_{n}(0)=-n$ for all $n \geqslant 1$. From time $t$ to time $t+1$, the first $t$ particles at locations $x_{1}(t), \ldots, x_{t}(t)$ are updated in parallel so that the $n$th particle jumps to $x_{n}(t+1)=x_{n}(t)+V_{n}(t)$, where $V_{n}(t)$ is distributed according to

$$
\mathbb{P}\left(V_{n}(t)=j\right)=\boldsymbol{\varphi}_{q, a_{n} a_{t}, 0}\left(j \mid x_{n-1}(t)-x_{n}(t)-1\right) .
$$

Additionally, the $(t+1)$ th particle jumps to the location $x_{t+1}(t+1)=-t-1+$ $W(t)$, where $W(t)$ is distributed according to the $q$-inverse Gaussian distribution with parameters $m=x_{t}(t+1)+t$ and $\theta$ equals $a_{t} a_{\circ}$ or $a_{t} / a_{\circ}$ depending on the parity of $t$ :

$$
\mathbb{P}(W(t)=k)=\left(a_{\circ}^{(-1)^{t-1}} a_{t}\right)^{k} \frac{(q ; q)_{m}}{(q ; q)_{k}(q ; q)_{m-k}} \frac{1}{Z_{m}\left(a_{\circ}^{(-1)^{t-1}} a_{t}\right)} .
$$

All participating random variables are independent. The particle system is depicted in Figure 11.

PROPOSITION 4.27. Let $\left(x_{n}(t)\right)_{1 \leqslant n \leqslant t}$ denote the positions of particles in the Geometric q-TASEP with activation (Definition 4.26). Let $\left(\lambda^{(t, n)}\right)_{1 \leqslant n \leqslant t}$ be a sequence of random partitions distributed according to the Markovian growth procedure described in Section 2.4, using transition operators $\mathcal{U}_{\text {row }}^{\llcorner}$and $\mathcal{U}^{\perp}$, and specializations $\rho_{\circ}$ and $\rho_{i}$ into single variables $a_{\circ}$ and $a_{i}$, respectively, for all $i \geqslant 1$. In particular, $\lambda^{(t, n)}$ is distributed according to the half-space $q$-Whittaker measure $\mathbb{P}_{\left(a_{1}, \ldots, a_{n}\right),\left(a_{0}, a_{n+1}, \ldots, a_{t}\right)}^{q}$. Then, for any admissible path $\omega \in \Omega$, we have

$$
\left(x_{n}(t)\right)_{(t, n) \in \omega} \stackrel{(d)}{=}\left(\lambda_{n}^{(t, n)}-n\right)_{(t, n) \in \omega} .
$$




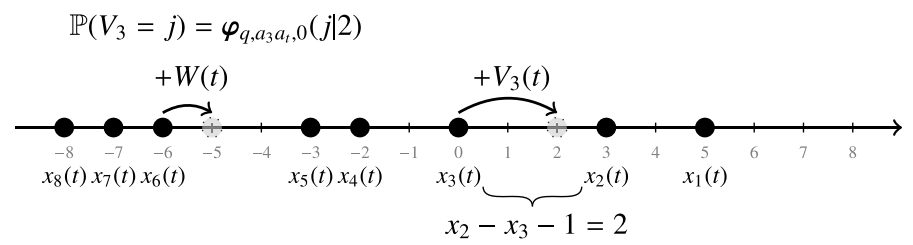

Figure 11. Configuration of particles in the Geometric $q$-TASEP with activation at time $t=5$. We illustrate a possible jump of the particle sitting at $x_{3}(t)$ and the activation of the sixth particle.

Proof. The two families have the same dynamics, according to Lemmas 4.15 and 4.22 .

\section{Half-space Hall-Littlewood process}

We assume now that $q=0$ and $t \in(0,1)$. We use again the same notations $P$, $Q, \mathcal{E}$ as before. These ( $P$ and $Q)$ are now called the Hall-Littlewood symmetric functions. We define the half-space Hall-Littlewood measure $\mathbb{P}_{\rho, \rho \downarrow}^{t}$ as the measure on partitions $\lambda \in \mathbb{Y}$ such that

$$
\mathbb{P}_{\rho, \rho^{\swarrow}}^{t}(\lambda)=\frac{P_{\lambda}(\rho) \mathcal{E}_{\lambda}\left(\rho^{\swarrow}\right)}{\Pi\left(\rho ; \rho^{\swarrow}\right) \Phi(\rho)},
$$

and denote by $\mathbb{P}_{\omega, \rho}^{t}$, for a sequence of specializations $\rho$, the half-space HallLittlewood process, that is, the $q=0$ degeneration of the half-space Macdonald process $\underset{\omega, \rho}{\mathbb{P}, t}$.

5.1. Observables and integral formulas. Consider a Hall-Littlewood measure where $\rho=\left(a_{1}, \ldots, a_{n}\right) \in(0,1)^{n}$, and $\rho^{\swarrow}=\rho(\alpha, \beta, \gamma)$ as defined in Section 2.2.4. We further assume that all the parameters $\alpha_{i}$ are such that $\max \left\{a_{i}\right\} \max \left\{\alpha_{j}\right\}<1$ so that the measure is well defined. We will be mostly interested in the distribution of the length of a Hall-Littlewood random partition $\lambda$, denoted by $\ell(\lambda)$ in the following.

COROLlaRY 5.1. Let $r$ be a positive integer. We have

$$
\begin{aligned}
& \mathbb{E}_{\left(a_{1}, \ldots, a_{n}\right), \rho}^{t}\left[e_{r}\left(t^{n-\ell(\lambda)-1}, t^{n-\ell(\lambda)-2}, \ldots, t^{0}\right)\right] \\
& \quad=\frac{1}{r !} \oint \frac{d z_{1}}{2 \mathbf{i} \pi} \cdots \oint \frac{d z_{r}}{2 \mathbf{i} \pi} \operatorname{det}\left[\frac{1}{t z_{k}-z_{l}}\right]_{k, l=1}^{r} \prod_{1 \leqslant i<j \leqslant r} \frac{1-t z_{i} z_{j}}{1-z_{i} z_{j}} \prod_{i=1}^{r} \frac{1-t z_{i}^{2}}{1-z_{i}^{2}}
\end{aligned}
$$




$$
\times \prod_{j=1}^{r}\left(\frac{1}{\Pi\left(z_{j} ; \rho\right)} \prod_{i=1}^{n}\left(\frac{t z_{j}-a_{i}}{z_{j}-a_{i}} \frac{1-z_{j} a_{i}}{1-t z_{j} a_{i}}\right)\right)
$$

where the positively oriented contours encircle $\left\{a_{1}, \ldots, a_{n}\right\}$ and no other singularity of the integrand.

Proof. This is the $q=0$ degeneration of Proposition 3.7. Indeed, when $q=0$,

$$
e_{r}\left(q^{\lambda_{1}} t^{n-1}, q^{\lambda_{2}} t^{n-2}, \ldots, q^{\lambda_{n}}\right)=e_{r}\left(t^{n-\ell(\lambda)-1}, \ldots, t^{0}\right) .
$$

Note that

$$
\sum_{r=0}^{n} u^{r} e_{r}\left(t^{n-\ell-1}, \ldots, t^{0}\right)=\prod_{i=0}^{n-\ell-1}\left(1+u t^{i}\right)=\frac{(-u ; t)_{\infty}}{\left(-u t^{n-\ell} ; t\right)_{\infty}} .
$$

The coefficient of $u^{r}$ can be extracted from the right-hand side using the $q$ binomial theorem (12):

$$
e_{r}\left(t^{n-\ell-1}, \ldots, t^{0}\right)=(-1)^{r}\left(t^{n-\ell}\right)^{r} \frac{\left(t^{\ell-n} ; t\right)_{r}}{(t ; t)_{r}} .
$$

REMARK 5.2. We may take a generating series of (95) and obtain a formula for $\mathbb{E}^{t}\left[\frac{(u ; t)_{\infty}}{\left(u t^{n-\ell(\lambda)} ; t\right)_{\infty}}\right]$. However, this is not a convenient observable to study the distribution of $\ell(\lambda)$ in asymptotic regimes where $n-\ell(\lambda)$ tends to $+\infty$, since it would require to scale $u$ to $+\infty$, and the prefactor $(u ; t)_{\infty}$ would diverge. A similar issue was encountered in [TW09] wherein the formula in Equation (2) was not directly amenable for asymptotic analysis (see also [BCS14, Theorem 5.5]). In [BBCW18], we show that in the special case where $\rho$ is trivial, it is possible to compute $\mathbb{E}^{t}\left[\frac{1}{\left(u t^{n-\ell(\lambda)} ; t^{2}\right)_{\infty}}\right]$ as a multiplicative functional of the Pfaffian Schur process, which can be written explicitly as contour integrals and the resulting formulas are amenable to asymptotic analysis.

We need to slightly change contours so as to obtain an integral formula for a better observable, following similar lines to [BCS14, BP18, Dim18]. For simplicity, we will focus on the case where the specialization $\rho$ is a pure alpha specialization (otherwise, we would need restrictive assumptions on the parameters $\left.\beta_{i}\right)$ of the form $\left(\alpha_{1}, \ldots, \alpha_{r}\right)$. Define the function

$$
\mathcal{G}^{t}(w)=\prod_{i=1}^{r}\left(1-\alpha_{i} w\right) \prod_{i=1}^{n} \frac{1-w a_{i}}{1-w / a_{i}}
$$




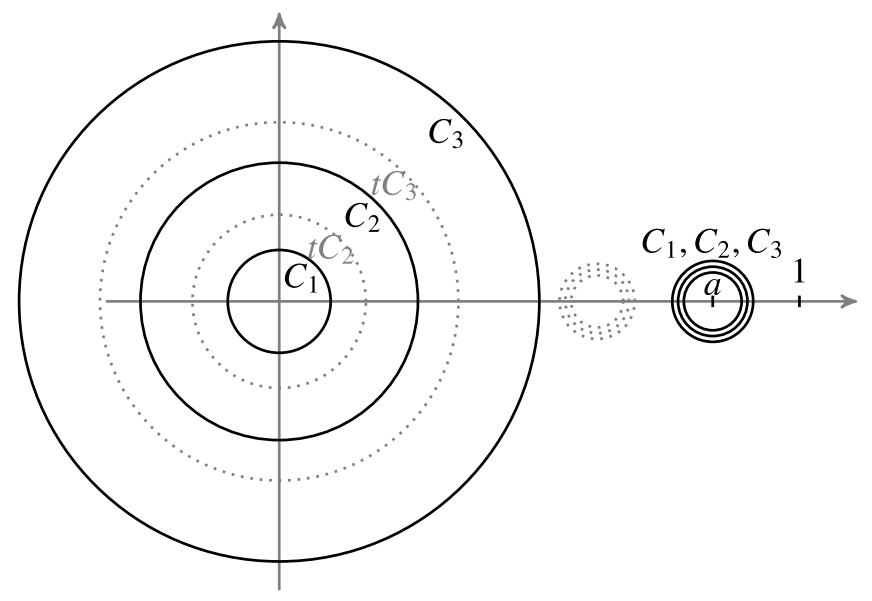

Figure 12. Possible choice of nested contours $C_{1}, C_{2}, C_{3}$ for Proposition 5.3 in a case where $a_{1}=a_{2}=a_{3}=a$. Each contour $C_{i}$ is the union of a circle around 0 and a circle around $a$. The dotted contours are the images of $C_{i}$ 's under $z \mapsto t z$.

so that (cf. (95))

$$
\frac{\mathcal{G}^{t}(w)}{\mathcal{G}^{t}(t w)}=\frac{1}{\Pi(w ; \rho)} \prod_{i=1}^{n} \frac{t w-a_{i}}{w-a_{i}} \frac{1-w a_{i}}{1-t w a_{i}} .
$$

Proposition 5.3. For any $m \geqslant 1$,

$$
\begin{aligned}
\mathbb{E}_{\left(a_{1}, \ldots, a_{n}\right), \rho}^{t}\left[\left(t^{n-\ell(\lambda)}\right)^{m}\right]= & t^{\frac{m(m-1)}{2}} \oint_{C_{1}} \frac{d z_{1}}{2 \mathbf{i} \pi} \cdots \oint_{C_{m}} \frac{d z_{m}}{2 \mathbf{i} \pi} \prod_{1 \leqslant i<j \leqslant m} \frac{z_{i}-z_{j}}{z_{i}-t z_{j}} \frac{1-t z_{i} z_{j}}{1-z_{i} z_{j}} \\
& \times \prod_{j=1}^{m} \frac{1}{z_{j}} \frac{1-t z_{j}^{2}}{1-z_{j}^{2}} \frac{\mathcal{G}^{t}\left(z_{j}\right)}{\mathcal{G}^{t}\left(t z_{j}\right)},
\end{aligned}
$$

where the positively oriented contours $C_{1}, \ldots, C_{m}$ all enclose 0 and $a_{i}$ and are contained in the open disk of radius 1 around zero, and the contours are nested in such a way that for $i<j$ the contour $C_{i}$ does not include any part of $t C_{j}$ (see Figure 12 for a possible choice of such contours).

Proof. Let $E_{r}=\mathbb{E}^{t}\left[(-1)^{r}\left(t^{n-\ell(\lambda)}\right)^{r} \frac{\left(t^{\ell(\lambda)-n} ; t\right)_{r}}{\left(t ; t_{r}\right.}\right]$. As a formal power series in $u$, we 
have that

$$
\begin{aligned}
\mathbb{E}^{t}\left[\frac{1}{\left(u t^{n-\ell(\lambda)} ; t\right)_{\infty}}\right] & =\sum_{k=0}^{\infty} \frac{u^{k}}{(t ; t)_{k}} \mathbb{E}^{t}\left[\frac{(u ; t)_{\infty}}{\left(u t^{n-\ell(\lambda)} ; t\right)_{\infty}}\right] \\
& =\sum_{r=0}^{n} \sum_{k=0}^{\infty} u^{r+k} \frac{(-1)^{r} E_{r}}{(t ; t)_{k}}=\sum_{m=0}^{\infty} u^{m} \sum_{k=0}^{m} \frac{(-1)^{k} E_{k}}{(t ; t)_{m-k}} .
\end{aligned}
$$

Using the Cauchy determinant evaluation in Corollary 5.1, we may write that for all $k$,

$$
(t ; t)_{k} E_{k}=\frac{t^{k(k-1) / 2}(t ; t)_{k}(-1)^{k}}{k !(1-t)^{k}} \oint \frac{d z_{1}}{2 \mathbf{i} \pi} \cdots \oint \frac{d z_{k}}{2 \mathbf{i} \pi} \prod_{i \neq j} \frac{z_{i}-z_{j}}{t z_{i}-z_{j}} \frac{F_{k}\left(z_{1}, \ldots, z_{k}\right)}{z_{1} \ldots z_{k}}
$$

where $F_{k}$ is a symmetric meromorphic function in $k$ variables such that $F_{k}$ has no pole in any variable in some disk around zero and for all $n<m, F_{m}\left(z_{1}, \ldots\right.$, $\left.z_{n}, 0, \ldots, 0\right)=F_{n}\left(z_{1}, \ldots, z_{n}\right)$, and the contours are as in the statement of the corollary. Using the symmetrization identity (89) to desymmetrize the integrand, we have

$$
(t ; t)_{k} E_{k}=(-1)^{k} t^{\frac{k(k-1)}{2}} \oint \frac{d z_{1}}{2 \mathbf{i} \pi} \cdots \oint \frac{d z_{k}}{2 \mathbf{i} \pi} \prod_{i<j} \frac{z_{i}-z_{j}}{t z_{i}-z_{j}} \frac{F_{k}\left(z_{1}, \ldots, z_{k}\right)}{z_{1} \ldots z_{k}}
$$

so that we can write

$$
\begin{aligned}
\mathbb{E}^{t} & {\left[\frac{1}{\left(u t^{n-\ell(\lambda)} ; t\right)_{\infty}}\right] } \\
= & \sum_{m=0}^{\infty} u^{m} \frac{t^{m(m-1) / 2}}{(t ; t)_{m}} \sum_{k=0}^{m}\left(\begin{array}{c}
m \\
k
\end{array}\right)_{t} \frac{t^{k(k-1) / 2}}{t^{m(m-1) / 2}} \\
& \times \oint_{C} \frac{d z_{1}}{2 \mathbf{i} \pi} \cdots \oint_{C} \frac{d z_{k}}{2 \mathbf{i} \pi} \prod_{i<j} \frac{z_{i}-z_{j}}{z_{i}-t z_{j}} \frac{F_{k}\left(z_{1}, \ldots, z_{k}\right)}{z_{1} \ldots z_{k}},
\end{aligned}
$$

where the contour $C$ encloses $\left\{a_{1}, \ldots, a_{n}\right\}$ and no other singularity.

LEMMA 5.4. For any $k \geqslant 0$, let $F_{k}$ be a symmetric meromorphic function in $k$ variables such that $F_{k}$ has no pole in any variable in some disk around zero, and for all $n<m, F_{m}\left(z_{1}, \ldots, z_{n}, 0, \ldots, 0\right)=F_{n}\left(z_{1}, \ldots, z_{n}\right)$. Then we have

$$
\oint_{C_{1}} \frac{d z_{1}}{2 \mathbf{i} \pi} \cdots \oint_{C_{m}} \frac{d z_{m}}{2 \mathbf{i} \pi} \prod_{i<j} \frac{z_{i}-z_{j}}{z_{i}-t z_{j}} \frac{F_{m}\left(z_{1}, \ldots, z_{m}\right)}{z_{1} \ldots z_{m}}
$$




$$
\begin{aligned}
= & \sum_{k=0}^{m}\left(\begin{array}{c}
m \\
k
\end{array}\right)_{t} t^{k(k-1) / 2-m(m-1) / 2} \oint_{C} \frac{d z_{1}}{2 \mathbf{i} \pi} \cdots \oint_{C} \frac{d z_{k}}{2 \mathbf{i} \pi} \\
& \times \prod_{i<j} \frac{z_{i}-z_{j}}{z_{i}-t z_{j}} \frac{F_{k}\left(z_{1}, \ldots, z_{k}\right)}{z_{1} \ldots z_{k}},
\end{aligned}
$$

where $C$ is an arbitrary contour not encircling 0 , and the contours $C_{1}, \ldots, C_{m}$ are defined as follows: Let $C_{0}$ be a small positively oriented circle around 0 , and let $r>t^{-1}$ be such that $t C$ does not intersect $r^{m} C_{0}$ and $r^{m} C_{0}$ does not encircle poles of $F_{m}$. Then $C_{j}$ is defined as the union of $r^{j} C_{0}$ and $C$.

Proof. The statement is the same as [BCS14, Lemma 4.21] except that the function $F$ was of the form $F_{k}\left(z_{1}, \ldots, z_{k}\right)=\prod_{i=1}^{k} f\left(z_{i}\right)$. The proof extends to our case without any modification.

Hence, extracting the coefficient of $u^{m}$ in (97) and using Lemma 5.4, we obtain

$$
\mathbb{E}^{t}\left[\left(t^{n-\ell(\lambda)}\right)^{m}\right]=t^{\frac{m(m-1)}{2}} \oint_{C_{1}} \frac{d z_{1}}{2 \mathbf{i} \pi} \cdots \oint_{C_{m}} \frac{d z_{m}}{2 \mathbf{i} \pi} \prod_{i<j} \frac{z_{i}-z_{j}}{z_{i}-t z_{j}} \frac{F_{m}\left(z_{1}, \ldots, z_{m}\right)}{z_{1} \ldots z_{m}}
$$

which concludes the proof of Proposition 5.3.

Proposition 5.5. For any $k \geqslant 1$,

$$
\begin{aligned}
\mathbb{E}_{\left(a_{1}, \ldots, a_{n}\right), \rho}^{t}\left[\left(t^{n-\ell(\lambda)}\right)^{k}\right] \\
=(t ; t)_{k} \sum_{\mu \vdash k} \frac{1}{m_{1} ! m_{2} ! \ldots} \oint_{\mathcal{C}} \frac{d w_{1}}{2 \mathbf{i} \pi} \cdots \oint_{\mathcal{C}} \frac{d w_{\ell(\mu)}}{2 \mathbf{i} \pi} \\
\quad \times \operatorname{det}\left(\frac{1}{w_{j}-w_{i} t^{\mu_{i}}}\right) \prod_{1 \leqslant a<b \leqslant \ell(\mu)} \frac{\left(t^{\mu_{a}} w_{a} w_{b}\right)_{\infty}\left(t^{\mu_{b}} w_{a} w_{b}\right)_{\infty}}{\left(w_{a} w_{b}\right)_{\infty}\left(t^{\mu_{a}+\mu_{b}} w_{a} w_{b}\right)_{\infty}} \\
\quad \times \prod_{j=1}^{\ell(\mu)} \frac{\left(t^{\mu_{j}} w_{j}^{2}\right)_{\infty}}{\left(w_{j}^{2}\right)_{\infty}} \frac{\left(t w_{i}^{2} ; t^{2}\right)_{\infty}}{\left(w_{i}^{2} t^{2 \mu_{i}+1} ; t^{2}\right)_{\infty}} \frac{\mathcal{G}^{t}\left(w_{j}\right)}{\mathcal{G}^{t}\left(t w_{j}\right)}
\end{aligned}
$$

where the positively oriented contour $\mathcal{C}$ contains $0, a_{i}$ 's and its image by multiplication by $t$, and is contained in the open disk of radius 1 around zero.

Proof. For

$$
\left(z_{1}, \ldots, z_{k}\right)=\left(w_{1}, t w_{1}, \ldots, t^{\lambda_{1}-1} w_{1}, w_{2}, \ldots, t^{\lambda_{2}-1} w_{2}, \ldots, t^{\lambda_{m}-1} w_{m}\right),
$$


an adaptation of Lemma 4.6 shows that

$$
\begin{aligned}
& \prod_{1 \leqslant a<b \leqslant k} \frac{1-t z_{a} z_{b}}{1-z_{a} z_{b}} \prod_{i=1}^{k} \frac{1-t z_{i}^{2}}{1-z_{i}^{2}} \\
= & \prod_{1 \leqslant a<b \leqslant m} \frac{\left(t^{\lambda_{a}} w_{a} w_{b} ; t\right)_{\infty}\left(t^{\lambda_{b}} w_{a} w_{b} ; t\right)_{\infty}}{\left(w_{a} w_{b} ; t\right)_{\infty}\left(t^{\lambda_{a}+\lambda_{b}} w_{a} w_{b} ; t\right)_{\infty}} \prod_{j=1}^{m} \frac{\left(t^{\lambda_{j}} w_{j}^{2} ; t\right)_{\infty}}{\left(w_{j}^{2} ; t\right)_{\infty}} \frac{\left(t w_{i}^{2} ; t^{2}\right)_{\infty}}{\left(w_{i}^{2} t^{2 \lambda_{i}+1} ; t^{2}\right)_{\infty}},
\end{aligned}
$$

and the left-hand side is symmetric in $z_{i}$. Then the formulas follow by expanding the nested contours to $C_{0, a}$, using (a slight variant of) Proposition 4.5 to collect the residues—see also [BCS14, Proposition 5.2]).

REMARK 5.6. Unlike the $q$-Whittaker case, it is not clear how to take a generating series of the above moment formulas. This is because the term

$$
\prod_{1 \leqslant a<b \leqslant k} \frac{\left(t^{\lambda_{a}} w_{a} w_{b}\right)_{\infty}\left(t^{\lambda_{b}} w_{a} w_{b}\right)_{\infty}}{\left(w_{a} w_{b}\right)_{\infty}\left(t^{\lambda_{a}+\lambda_{b}} w_{a} w_{b}\right)_{\infty}}
$$

is typically of size $e^{c k^{2}}$ as $k$ grows. In the $q$-Whittaker case, a similar factor is present as well (cf. (85)), but one could argue that only finitely many integral terms are nonzero when taking the moment generating series. This would not be the case here.

5.2. Half-space stochastic six-vertex model. Recall the definition of the half-space stochastic six-vertex model (Definition 1.2). The height function in the half-space stochastic six-vertex model is related to length of partitions in the half-space Hall-Littlewood processes in the following way.

THEOREM 5.7 [BBCW18, Corollary 4.5]. Let $n \geqslant 1$ and $\omega_{n} \in \Omega$ be the path in $\Omega$, which travels from $(+\infty, 0)$ to $(n, 0)$, goes vertically to $(n, n)$ and diagonally to $(0,0)$ (see Figure 13). Let $\rho_{n}$ be a sequence of specializations such that edges $(n, i-1) \rightarrow(n, i)$ are labeled by the single variable specialization into $a_{i}$ and the diagonal specialization is empty. Consider a sequence of partitions $\lambda$ distributed according to the half-space Hall-Littlewood process $\mathbb{P}_{\omega_{n}, \rho_{n}}^{t}$. Then we have

$$
\left(\ell\left(\lambda^{v}\right)\right)_{v \in \omega_{n}} \stackrel{(d)}{=}(\mathfrak{h}(v))_{v \in \tilde{\omega}_{n}},
$$

where $\tilde{\omega}_{n}$ the path in $\mathbb{Z}^{2}$ obtained by reflecting $\omega$ with respect to the quadrant diagonal. 


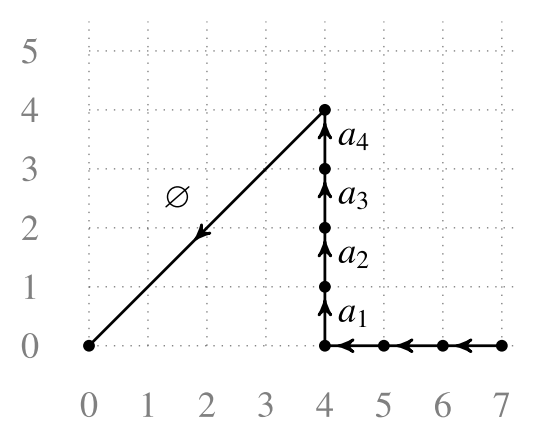

Figure 13. The path $\omega_{n}$ for $n=4$.

In particular, for any $1 \leqslant x \leqslant y, \mathfrak{h}(x, y)$ has the same distribution as $\ell(\lambda)$, where $\lambda$ is distributed according to the half-space Hall-Littlewood measure $\mathbb{P}_{\left(a_{1}, \ldots, a_{x}\right),\left(a_{x+1}, \ldots, a_{y}\right)}^{t}$.

Note that we need to perform a reflection of the path $\omega_{n}$ simply because, in order to be consistent with earlier literature, we have defined our halfspace stochastic six-vertex model in the upper half-quadrant while half-space Macdonald processes are indexed by paths in the lower half-quadrant. Moreover, we expect the result should hold for any admissible path in $\Omega$-see [BBCW18, Remark 4.6].

COROLlary 5.8. For integers $1 \leqslant x \leqslant y$ and $k \geqslant 1$,

$$
\begin{aligned}
\mathbb{E}^{t}\left[t^{-k \mathfrak{h}(x, y)}\right] & t^{\frac{k(k-1)}{2}} \oint_{C_{1}} \frac{d z_{1}}{2 \mathbf{i} \pi} \cdots \oint_{C_{k}} \frac{d z_{k}}{2 \mathbf{i} \pi} \prod_{1 \leqslant i<j \leqslant k} \frac{z_{i}-z_{j}}{z_{i}-t z_{j}} \frac{1-t z_{i} z_{j}}{1-z_{i} z_{j}} \\
& \times \prod_{j=1}^{k}\left(\frac{1}{z_{j}} \frac{1-t z_{j}^{2}}{1-z_{j}^{2}} \prod_{i=1}^{y} \frac{1-a_{i} z_{j}}{1-t a_{i} z_{j}} \prod_{i=1}^{x} \frac{z_{j}-a_{i} / t}{z_{j}-a_{i}}\right),
\end{aligned}
$$

where the positively oriented contours $C_{1}, \ldots, C_{m}$ all enclose 0 and $a_{i}$ 's, and are contained in the open disk of radius 1 around zero, and the contours are nested in such a way that for $i<j$ the contour $C_{i}$ does not include any part of $t C_{j}$.

Proof. This is a direct consequence of Proposition 5.3 and Theorem 5.7. 


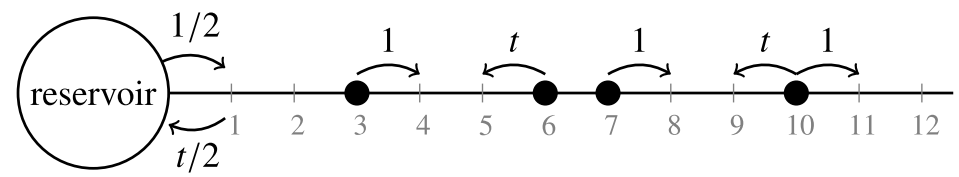

Figure 14. Jump rates in the half-line ASEP.

\subsection{Scaling limit to half-line ASEP.}

DEFINITION 5.9. The half-line ASEP is an interacting particle system on $\mathbb{Z}_{>0}$, where each site is occupied by at most one particle. It is a continuous-time Markov process on the space of particle configurations, such that each particle jumps by one to the right at rate $\mathbf{p}$ and to the left at rate $\mathbf{q}$, with $\mathbf{q}<\mathbf{p}$, provided the target site is empty. At the origin, a reservoir of particles injects a particle at site 1 (whenever it is empty) at rate $\boldsymbol{\alpha}$ and removes a particle from site 1 (whenever it is occupied) at rate $\gamma$. We will further assume that initially all sites are empty and the parameters are chosen as $\mathbf{p}=1, \mathbf{q}=t, \boldsymbol{\alpha}=1 / 2, \boldsymbol{\gamma}=t / 2$-see Figure 14 for an illustration. We refer the reader to [BBCW18] for the reasons behind this specific choice of parameters. We define the current at site $x$ by

$$
N_{x}(\tau)=\sum_{i=x}^{\infty} \eta_{i}(\tau),
$$

where $\eta_{i}(\tau) \in\{0,1\}$ is the occupation variable at site $i$ and time $\tau$.

The stochastic six-vertex model in a half-quadrant is a discretization of halfline ASEP in the following sense. Consider the six-vertex model in a halfquadrant where $a_{x} \equiv a$, and scale $a$ as

$$
a=1-\frac{(1-t) \varepsilon}{2}, \quad \varepsilon \underset{\varepsilon>0}{\longrightarrow} 0
$$

so that to first order in $\varepsilon$,

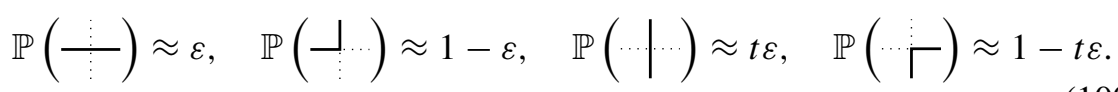

Moreover, we rescale $n$ as $n=\tau \varepsilon^{-1}$ with finite $\tau>0$.

PROPOSITION 5.10 [BBCW18, Proposition 5.2]. Under the scalings above and the boundary and initial conditions as in Definition 5.9, for any $x \in\{1,2, \ldots\}$,

$$
n-x-\mathfrak{h}(n-x, n) \underset{\varepsilon \rightarrow 0}{\Longrightarrow} N_{x}(\tau),
$$

where $\mathfrak{h}$ is defined in Definition 1.2 and $N_{x}(\tau)$ is defined in Definition 5.9. 
It would be interesting to take a limit of the moment formula from Corollary 5.8 in order to deduce formulas for the current in half-line ASEP. However, the choice of contours above-including $a_{i}$ but excluding 1 -does not allow us to take this limit directly. One would need to expand the contours in (99) to become a circle with radius larger than 1 . During this deformation of contours, one would encounter the same poles for $z_{i}=t z_{j}$ when $i<j$ as in the proof of Proposition 5.5 , but also additional poles when $z_{i}= \pm 1$ and when $z_{i}=1 / z_{j}$. It is not clear how to rearrange the contribution of all the corresponding residues and we do not know an analogue of Proposition 4.5 in that setting. It is worth mentioning that in the limit when $t$ goes to 1, an analogue of Proposition 4.5 was proposed in [BBC16] as Conjecture 5.2 with Pfaffians replacing the determinants.

In the attempt to prove [BBC16, Conjecture 5.2] proposed in [BBC16, Section 7.3], an essential step is to make use of symmetrization identities over the hyperoctahedral group $B C_{k}$ instead of the symmetric group $\mathcal{S}_{k}$ as in the proof of Proposition 4.5 (see [BBC16, Section 7] for details). The following identity was proved in [Ven15, Eq. (5)],

$$
\begin{aligned}
& \sum_{\sigma \in B C_{k}} \sigma\left(\prod_{1 \leqslant i<j \leqslant k} \frac{1-t \frac{z_{j}}{z_{i}}}{1-\frac{z_{j}}{z_{i}}} \frac{1-t \frac{1}{z_{i} z_{j}}}{1-\frac{1}{z_{i} z_{j}}} \prod_{j=1}^{k} \frac{\left(1-a \frac{1}{z_{j}}\right)\left(1-b \frac{1}{z_{j}}\right)}{1-\frac{1}{z_{j}^{2}}}\right) \\
& =\prod_{j=1}^{k} \frac{\left(1-t^{j}\right)\left(1-a b t^{j-1}\right)}{1-t}
\end{aligned}
$$

where $\sigma$ is a signed permutation acting by permutation and inversion of variables $z_{i}$. Its degeneration as $t$ goes to 1 was an important tool to arrive at [BBC16, Conjecture 5.2].

Taking $a=-b=\sqrt{t}$, and inverting variables in (101), we obtain

$$
\sum_{\sigma \in B C_{k}} \sigma\left(\prod_{1 \leqslant i<j \leqslant k} \frac{z_{j}-t z_{i}}{z_{j}-z_{i}} \frac{1-t z_{i} z_{j}}{1-z_{i} z_{j}} \prod_{j=1}^{k} \frac{1-t z_{j}^{2}}{1-z_{j}^{2}}\right)=\frac{\left(t^{2}, t^{2}\right)_{k}}{(1-t)^{k}}
$$

We expect that this BC-type symmetrization identity should play a role when moving all contours in (96) to a circle with radius larger than 1 . This further suggests that the appropriate moment generating series to consider is

$$
\sum_{k=0}^{+\infty} \frac{u^{k} \mathbb{E}^{t}\left[t^{k(n-\ell(\lambda))}\right]}{\left(t^{2}, t^{2}\right)_{k}}=\mathbb{E}^{t}\left[\frac{1}{\left(u t^{n-\ell(\lambda)} ; t^{2}\right)_{\infty}}\right] .
$$

It was confirmed in [BBCW18, Proposition 3.3] that this quantity indeed admits a Fredholm Pfaffian representation at least in the special case where the 
specializations of the half-space Hall-Littlewood measure are $\rho=\left(a_{1}, \ldots, a_{x}\right)$ and $\rho^{\swarrow}=\varnothing$. Allowing a more general $\rho^{\swarrow}$ would allow us to study $\mathfrak{h}(x, y)$ for $y>x$, but we cannot presently generalize the results of [BBCW18].

\section{Half-space Whittaker processes}

6.1. Whittaker functions. Define the class-one $\mathfrak{g l}_{n}(\mathbb{R})$-Whittaker functions via their integral representations [Giv97]

$$
\psi_{\lambda}(x)=\int_{\mathbb{R}^{\frac{n(n-1)}{2}}} \prod_{k=1}^{n-1} \prod_{i=1}^{k} d x_{k, i} \exp \left(\mathcal{F}_{\lambda}(X)\right),
$$

where $\lambda=\left(\lambda_{1}, \ldots, \lambda_{n}\right) \in \mathbb{C}^{n}, x=\left(x_{1}, \ldots, x_{n}\right), X=\left(x_{k, i}: 1 \leqslant i \leqslant k \leqslant n\right)$, $x_{n, i}=x_{i}$ and

$$
\mathcal{F}_{\lambda}(X)=\mathbf{i} \sum_{k=1}^{n} \lambda_{k}\left(\sum_{i=1}^{k} x_{k, i}-\sum_{i=1}^{k-1} x_{k-1, i}\right)-\sum_{k=1}^{n-1} \sum_{i=1}^{k}\left(e^{x_{k, i}-x_{k+1, i}}+e^{x_{k+1, i+1}-x_{k, i}}\right) .
$$

Note that unlike previous sections, $\lambda$ does not denote a partition but a vector in $\mathbb{C}^{n}$. Furthermore, we will see that Whittaker functions play a role similar to $q$-Whittaker and Macdonald functions in previous sections, but in order to be consistent with notations commonly used in the literature, the role of the index $\lambda$ and the variable $x$ are switched (for instance, in Proposition 6.5 the index $\lambda$ in $\psi_{\lambda}(x)$ corresponds to the variable $x$ in $P_{\lambda}(x)$ and vice versa). Whittaker functions satisfy the following two integral identities.

Proposition 6.1 ([Sta01], [OSZ14, Corollaries 3.6 and 3.7]). Suppose $u>0$ and $\lambda, v \in \mathbb{C}^{n}$ with $\mathfrak{R e}\left[\lambda_{i}+v_{j}\right]>0$ for all $1 \leqslant i, j \leqslant n$. Then

$$
\int_{\mathbb{R}^{n}} d x e^{-u e^{-x_{n}}} \psi_{\mathbf{i} \lambda}(x) \psi_{\mathbf{i v}}(x)=u^{-\sum_{j=1}^{n}\left(\lambda_{j}+v_{j}\right)} \prod_{1 \leqslant i, j \leqslant n} \Gamma\left(\lambda_{i}+v_{j}\right)
$$

and

$$
\int_{\mathbb{R}^{n}} d x e^{-u e^{x_{1}}} \psi_{-\mathbf{i} \lambda}(x) \psi_{-\mathbf{i v}}(x)=u^{-\sum_{j=1}^{n}\left(\lambda_{j}+v_{j}\right)} \prod_{1 \leqslant i, j \leqslant n} \Gamma\left(\lambda_{i}+v_{j}\right) .
$$

Define the Sklyanin measure $\mathfrak{m}_{n}(\xi)$ as

$$
\mathfrak{m}_{n}(\xi)=\frac{1}{(2 \pi)^{n} n !} \prod_{\substack{i, j=1 \\ i \neq j}}^{n} \frac{1}{\Gamma\left(\mathbf{i} \xi_{i}-\mathbf{i} \xi_{j}\right)}
$$


We have the following orthogonality relations, to be understood in a weak sense (see [GLO08, Theorem 2.1], [STS93]). For all $\lambda, \mu, x, y \in \mathbb{R}^{n}$,

$$
\int_{\mathbb{R}^{n}} \overline{\psi_{\lambda}(x)} \psi_{\mu}(x) d x=\frac{1}{n ! \mathfrak{m}_{n}(\lambda)} \sum_{\sigma \in \mathcal{S}_{n}} \delta(\lambda-\sigma(\mu))
$$

and

$$
\int_{\mathbb{R}^{n}} \overline{\psi_{\lambda}(x)} \psi_{\lambda}(y) \mathfrak{m}_{n}(\lambda) d \lambda=\delta(x-y) .
$$

Then, Proposition 6.1 yields the following. Let $\mathbb{H}$ denote the complex upper halfplane $\mathbb{H}=\{z \in \mathbb{C}: \mathfrak{I m}[z]>0\}$.

Proposition 6.2. For $u>0$ and $w \in \mathbb{C}^{n}, w_{i} \in \mathbb{H}, 1 \leqslant i \leqslant n$,

$$
e^{-u e^{-x_{n}}} \psi_{w}(x)=\int_{\mathbb{R}^{n}} d \xi \mathfrak{m}_{n}(\xi) u^{\mathbf{i} \sum_{k=1}^{n}\left(w_{i}+\xi_{i}\right)} \prod_{1 \leqslant i, j \leqslant n} \Gamma\left(-\mathbf{i} \xi_{i}-\mathbf{i} w_{j}\right) \psi_{-\xi}(x)
$$

and

$$
e^{-u e^{x_{1}}} \psi_{-w}(x)=\int_{\mathbb{R}^{n}} d \xi \mathfrak{m}_{n}(\xi) u^{\mathbf{i} \sum_{k=1}^{n}\left(w_{i}+\xi_{i}\right)} \prod_{1 \leqslant i, j \leqslant n} \Gamma\left(-\mathbf{i} \xi_{i}-\mathbf{i} w_{j}\right) \psi_{\xi}(x) .
$$

Define an operator $\mathbf{B}_{n}^{u}$ acting on functions $\mathbb{H}^{n} \rightarrow \mathbb{C}$ by

$$
\mathbf{B}_{n}^{u} f(w)=\int_{\mathbb{R}^{n}} d \xi \mathfrak{m}_{n}(\xi) u^{\mathbf{i} \sum_{k=1}^{n}\left(w_{i}+\xi_{i}\right)} \prod_{1 \leqslant i, j \leqslant n} \Gamma\left(-\mathbf{i} \xi_{i}-\mathbf{i} w_{j}\right) f(-\xi) .
$$

Define as well a very similar operator $\overline{\mathbf{B}}_{n}^{u}$ acting on functions $(-\mathbb{H})^{n} \rightarrow \mathbb{C}$ by

$$
\overline{\mathbf{B}}_{n}^{u} f(w)=\int_{\mathbb{R}^{n}} d \xi \mathfrak{m}_{n}(\xi) u^{\mathbf{i} \sum_{k=1}^{n}\left(-w_{i}+\xi_{i}\right)} \prod_{1 \leqslant i, j \leqslant n} \Gamma\left(-\mathbf{i} \xi_{i}+\mathbf{i} w_{j}\right) f(\xi),
$$

that is, $\overline{\mathbf{B}}_{n}^{u}$ is a composition of $\mathbf{B}_{n}^{u}$ and $w \mapsto-w$. We may rewrite Equations (107) and (108) as $e^{-u e^{-x_{n}}} \psi_{w}(x)=\mathbf{B}^{u} \psi_{w}(x)$ and $e^{-u e^{x_{1}}} \psi_{w}(x)=\overline{\mathbf{B}}^{u} \psi_{w}(x)$ (dropping the index $n$ ). The operator $\overline{\mathbf{B}}^{u}$ is referred to as dual Baxter operator in [GLO08]. It was shown in [BCR15] that the eigenrelation (107) for $\mathbf{B}^{u}$ arises as a $t=0, q \rightarrow 1$ limit of the eigenrelation (50) for Noumi's $q$-integral operator $\mathbf{N}^{z}$. We expect that eigenrelation (108) for $\overline{\mathbf{B}}^{u}$ similarly arises as the limit of the eigenrelation for $\mathbf{M}^{z}$ from Proposition 3.27. 


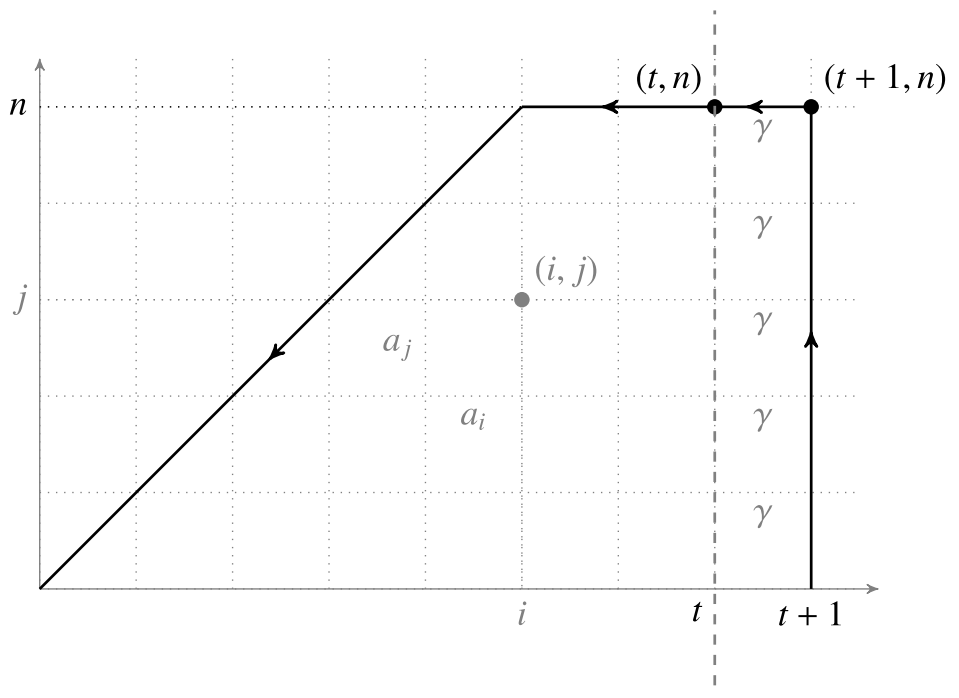

Figure 15. The path $\omega$ considered in Section 6.2.

6.2. Half-space Whittaker process with Plancherel specialization. In this section, we are interested in the limit of the half-space $q$-Whittaker process when $q$ goes to 1. For applications, it would be natural to focus on the case when specializations are all pure alpha, that is the setting of Section 4.2. However, we know from the study of the full-space Whittaker process that some technical difficulties arise with this choice of specializations (see [BC14, Section 4.2]). More precisely, some tail estimates about integrals involving Whittaker functions are necessary to justify the convergence of the $q$-Whittaker process, and these seem difficult to establish. However, all these estimates are much easier to prove when one includes a Plancherel specialization (see [BC14, Section 4.1]). Thus, following an idea already present in [BCFV15], we will consider a half-space $q$-Whittaker process, where we add to the specialization $\left(a_{\circ}, a_{n+1}, \ldots, a_{t}\right)$ of $\mathcal{E}_{\lambda}$ some Plancherel component $\gamma$.

Consider a half-space $q$-Whittaker process indexed by a path $\omega$ as in Figure 15 , with the following choice of specializations. Fix $1 \leqslant n \leqslant t$. For all $i \leqslant t$ and any $j$, assume that edges $(i-1, j) \leftarrow(i, j)$ are labeled by single variable specialization $a_{i}$, for any $i, j$, edges $(i, j-1) \rightarrow(i, j)$ are labeled by single variable specialization $a_{j}$, the diagonal edge is labeled by specialization $a_{\circ}$ andunlike in Section 4.2-edges $(t, j) \leftarrow(t+1, j)$ are labeled by the Plancherel specialization $\gamma$.

Let us denote for $1 \leqslant m \leqslant n, \lambda^{(m)}:=\lambda^{(t+1, m)}$. The probability of the sequence 


$$
\begin{aligned}
& \lambda^{(1)} \prec \ldots \prec \lambda^{(n)} \text { is } \\
& \quad \mathbb{P}_{\omega, \rho}^{q}\left(\lambda^{(1)}, \ldots, \lambda^{(n)}\right)=\frac{\mathcal{E}_{\lambda^{(n)}}\left(\left(a_{\circ}, a_{n+1}, \ldots, a_{t}\right), \gamma\right) P_{\lambda^{(n)} / \lambda^{(n-1)}}\left(a_{n}\right) \ldots P_{\lambda^{(1)}}\left(a_{1}\right)}{\Pi\left(a_{1}, \ldots, a_{n} ;\left(a_{\circ}, a_{n+1}, \ldots, a_{t}\right), \gamma\right) \Phi\left(a_{1}, \ldots, a_{n}\right)}
\end{aligned}
$$

In particular, $\lambda^{(n)}$ is distributed according to the half-space $q$-Whittaker measure,

$$
\begin{aligned}
& \mathbb{P}_{\left(a_{1}, \ldots, a_{n}\right),\left(\left(a_{\circ}, a_{n+1}, \ldots, a_{t}\right), \gamma\right)}^{q}\left(\lambda^{(n)}\right) \\
& \quad=\frac{P_{\lambda^{(n)}}\left(a_{1}, \ldots, a_{n}\right) \mathcal{E}_{\lambda^{(n)}}\left(\left(a_{\circ}, a_{n+1}, \ldots, a_{t}\right), \gamma\right)}{\Pi\left(a_{1}, \ldots, a_{n} ;\left(a_{\circ}, a_{n+1}, \ldots, a_{t}\right), \gamma\right) \Phi\left(a_{1}, \ldots, a_{N}\right)} .
\end{aligned}
$$

To go from $q$-Whittaker to Whittaker processes, we use the following scalings:

$$
\begin{gathered}
q=e^{-\varepsilon}, \quad a_{j}=e^{-\varepsilon \alpha_{j}}, \quad a_{\circ}=e^{-\varepsilon \alpha_{\circ}}, \quad \gamma=\tau \varepsilon^{-2} \\
\lambda_{j}^{(m)}=\tau \varepsilon^{-2}+(t+m+1-2 j) \varepsilon^{-1} \log \varepsilon^{-1}+\varepsilon^{-1} T_{j}^{(m)}, \quad \forall 1 \leqslant m \leqslant n .
\end{gathered}
$$

REMARK 6.3. Based on the analogy between half-space Macdonald processes and usual Macdonald processes, our random variable $T_{j}^{(m)}$ corresponds to the random variable denoted by $T_{m, j}$ in the context of the $\alpha$-Whittaker process [BC14, Section 4.2].

Let us recall some convergence results from [BC14].

LeMMA 6.4 [BC14, Proposition 4.1.9]. For any $M \in \mathbb{R}$,

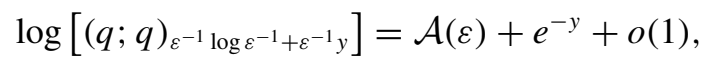

where $\mathcal{A}(\varepsilon)=-\varepsilon^{-1} \frac{\pi^{2}}{6}-\frac{1}{2} \log \frac{\varepsilon}{2 \pi}$, and for $k>1$,

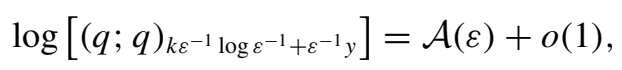

where the error $o(1)$ in (113) and (114) goes to 0 uniformly for $y>M$ as $\varepsilon \rightarrow 0$. Moreover, for any $y \in \mathbb{R}$ and $k \geqslant 1$, we have the inequality

$$
\log (q ; q)_{k \varepsilon^{-1} \log \left(\varepsilon^{-1}\right)+\varepsilon^{-1} y} \geqslant \mathcal{A}(\varepsilon)+\varepsilon^{-1} e^{-y+k \log (\varepsilon)}-c(\varepsilon),
$$

where $c(\varepsilon) \rightarrow 0$ uniformly in $y$.

We recall that (with $t=0$ )

$$
\Pi\left(a_{1}, \ldots, a_{n} ; b_{1}, \ldots, b_{k}\right)=\prod_{i=1}^{n} \prod_{j=1}^{k} \frac{1}{\left(a_{i} b_{j} ; q\right)_{\infty}}, \Phi\left(a_{1}, \ldots, a_{n}\right)
$$




$$
=\prod_{1 \leqslant i<j \leqslant n} \frac{1}{\left(a_{i} a_{j} ; q\right)_{\infty}} .
$$

Noting that by the convergence of the $q$-Gamma function to the Gamma function (see (14)),

$$
\left(e^{-\varepsilon \tilde{x}} ; e^{-\varepsilon}\right)_{\infty}=\varepsilon^{1-\tilde{x}} \frac{1}{\Gamma(\tilde{x})} e^{\mathcal{A}(\varepsilon)+o(1)}
$$

so that (see [BCFV15, Lemma 4.9]) with the scalings $a_{i}=q^{\alpha_{i}}, b_{i}=q^{\beta_{i}}$,

$$
\begin{aligned}
\Pi\left(a_{1}, \ldots, a_{n} ;\left(b_{1}, \ldots, b_{k}\right), \gamma\right)= & \left(e^{\tau t \varepsilon^{-2}} e^{-\varepsilon^{-1} \tau \sum_{j=1}^{n} \alpha_{j}} \prod_{i=1}^{k} \prod_{j=1}^{n} \frac{1}{e^{\mathcal{A}(\epsilon)} \epsilon^{1-\beta_{i}-\alpha_{j}}}\right) \\
& \times e^{\tau \sum_{j=1}^{n} \alpha_{j}^{2} / 2} \prod_{i=1}^{k} \prod_{j=1}^{n} \Gamma\left(\beta_{i}+\alpha_{j}\right) e^{o(1)},
\end{aligned}
$$

where the $o(1)$ error goes to zero as $\epsilon \rightarrow 0$. Similarly, we have

$$
\begin{aligned}
\Phi\left(a_{1}, \ldots, a_{n}\right) & =\prod_{1 \leqslant i<j \leqslant n} \varepsilon^{\alpha_{i}+\alpha_{j}-1} e^{-\mathcal{A}(\varepsilon)} \Gamma\left(\alpha_{i}+\alpha_{j}\right) e^{o(1)} \\
& =\varepsilon^{(n-1) \sum \alpha_{i}} \varepsilon^{-\frac{n(n-1)}{2}} e^{-\frac{n(n-1)}{2} \mathcal{A}(\varepsilon)} \prod_{1 \leqslant i<j \leqslant n} \Gamma\left(\alpha_{i}+\alpha_{j}\right) e^{o(1)} .
\end{aligned}
$$

Proposition 6.5 [BC14, Theorem 4.1.7]. Fix $1 \leqslant n \leqslant t$, the scalings $q=e^{-\varepsilon}$, $\lambda_{j}=\tau \varepsilon^{-2}+(t+n+1-2 j) \varepsilon^{-1} \log \varepsilon^{-1}+\varepsilon^{-1} x_{k}$ and $z_{j}=e^{\mathbf{i} \varepsilon v_{j}}$ for $1 \leqslant j \leqslant n$, and let

$$
\psi_{v}^{\varepsilon}(x)=\varepsilon^{\frac{n(n-1)}{2}} \varepsilon^{t \sum_{k=1}^{n} \mathbf{i} v_{k}} e^{\tau \varepsilon^{-1} \sum_{k=1}^{n} \mathbf{i} v_{k}} e^{\frac{n(n-1)}{2} \mathcal{A}(\varepsilon)} P_{\lambda}(z) .
$$

Then, for all $v \in \mathbb{C}^{n}$, as $\varepsilon \rightarrow 0, \psi_{v}^{\varepsilon}(x)$ converges to $\psi_{v}(x)$ uniformly for $x$ in a compact set.

Proof. The scalings above are slightly different from the setting of [BC14, Theorem 4.1.7], but the result is obtained using the same arguments as in Step 2 of the proof of [BC14, Lemma 4.1.25].

LeMma 6.6 [BCFV15, Lemma 4.8]. Fix any compact subset $D \subset \mathbb{R}^{n(n+1) / 2}$. Then, under scalings (111) and (112),

$$
\begin{aligned}
& P_{\lambda^{(n)} / \lambda^{(n-1)}}\left(a_{n}\right) P_{\lambda^{(n-1)} / \lambda^{(n-2)}}\left(a_{n-1}\right) \ldots P_{\lambda^{(1)}}\left(a_{1}\right) \\
& \quad=e^{-\frac{n(n-1)}{2} \mathcal{A}(\varepsilon)} \varepsilon^{t \sum_{k=1}^{n} \alpha_{k}} e^{-\varepsilon^{-1} \tau \sum_{j=1}^{n} \alpha_{j}} e^{\mathcal{F}_{\mathrm{i} \alpha_{1}, \ldots, \mathrm{i} \alpha_{n}}(T)} e^{o(1)},
\end{aligned}
$$

where the error o(1) goes to zero as $\varepsilon$ to 0 uniformly with respect to $\left(T_{j}^{m}\right)_{1 \leqslant j \leqslant m \leqslant n} \in D$. 
Proposition 6.5 and Lemma 6.6 correct a mistake in the exponent in front of $\mathcal{A}$ present in [BC14], as pointed out in [BCR15].

We define now the Whittaker analogue of the symmetric function $\mathcal{E}_{\lambda}$.

Definition 6.7. We define for $\tau>0, \alpha \in \mathbb{R}^{k}$ and $x \in \mathbb{R}^{n}$,

$$
\begin{aligned}
\mathcal{T}_{\alpha}^{\tau}(x)= & \int_{(\mathbb{R}+\mathbf{i} a)^{n}} \psi_{-v}(x) e^{-\tau \sum_{j=1}^{n} v_{j}^{2} / 2} \prod_{i=1}^{k} \prod_{j=1}^{n} \Gamma\left(\alpha_{i}-\mathbf{i} v_{j}\right) \\
& \times \prod_{1 \leqslant i<j \leqslant n} \Gamma\left(-\mathbf{i}\left(v_{i}+v_{j}\right)\right) \mathfrak{m}_{n}(\nu) d \nu,
\end{aligned}
$$

where $a>0$ and $\alpha_{i}+a>0$ for all $1 \leqslant i \leqslant k$.

PROPOSITION 6.8. Let $\tau>0, n, k \in \mathbb{Z}_{>0}$, and fix a compact subset $\mathbb{K} \subset \mathbb{R}^{n}$. Then, under the scalings $q=e^{-\varepsilon}, \lambda_{j}=\tau \varepsilon^{-2}+(2 n+k-2 j) \varepsilon^{-1} \log \left(\varepsilon^{-1}\right)+\varepsilon^{-1} x_{j}$ for $1 \leqslant j \leqslant n, a_{i}=q^{\alpha_{i}}$ for $1 \leqslant i \leqslant k$ and $\gamma=\tau \varepsilon^{-2}$,

$$
\mathcal{E}_{\lambda}\left(\left(a_{1}, \ldots, a_{k}\right), \gamma\right)=\varepsilon^{n} e^{\tau n \varepsilon^{-2}}\left(\prod_{i=1}^{k} \frac{1}{e^{\mathcal{A}(\varepsilon)} \varepsilon^{1-\alpha_{i}}}\right)^{n} \mathcal{T}_{\alpha}^{\tau}(x) e^{o(1)},
$$

where $\mathcal{E}_{\lambda}$ is specialized into the union of the pure alpha specialization $\left(a_{1}, \ldots\right.$, $a_{k}$ ) and a Plancherel specialization with parameter $\gamma$, and the o(1) error goes to zero uniformly as $\varepsilon \rightarrow 0$ for $x \in \mathbb{K}$.

Proof. We adapt the proof of [BCFV15, Lemma 4.10]. Recall that using the torus scalar product $\langle\langle\cdot, \cdot\rangle\rangle$ from Section 2.2.5, we may write for $\varepsilon>0$ and $a>0$,

$$
\mathcal{E}_{\lambda}(x)=\frac{1}{\left\langle\left\langle P_{\lambda}, P_{\lambda}\right\rangle\right\rangle} \int_{\left(e^{-\varepsilon a} \mathbb{T}\right)^{n}} P_{\lambda}\left(z^{-1}\right) \Pi(z, x) \Phi(z) \mathfrak{m}_{n}^{q, t}(z) \prod_{i=1}^{n} \frac{d z_{i}}{z_{i}} .
$$

Under the scalings we consider, we have $\left\langle\left\langle P_{\lambda}, P_{\lambda}\right\rangle\right\rangle=\left\langle P_{\lambda}, P_{\lambda}\right\rangle^{\prime}=e^{o(1)}$ as in [BC14, Lemma 4.1.25]. In the following, we will use the change of variables $z_{j}=\exp \left(\mathbf{i} \varepsilon v_{j}\right)$. Fix a compact subset $V \subset \mathbb{R}^{N}$. Then,

$$
\Pi\left(z_{1}, \ldots, z_{n} ;\left(a_{1}, \ldots, a_{k}\right), \gamma\right)=E_{\Pi} \prod_{i=1}^{k} \prod_{j=1}^{n} \Gamma\left(\alpha_{i}-\mathbf{i} v_{j}\right) e^{-\tau \sum_{j=1}^{n} v_{j}^{2} / 2} e^{o(1)},
$$

where $E_{\Pi}:=\prod_{i=1}^{k} \prod_{j=1}^{n} e^{-\mathcal{A}(\epsilon)} \epsilon^{-1+\alpha_{i}-\mathbf{i} v_{j}}$,

$$
\Phi\left(z_{1}, \ldots, z_{n}\right)=E_{\Phi} \prod_{1 \leqslant i<j \leqslant n} \Gamma\left(-\mathbf{i} v_{i}-\mathbf{i} v_{j}\right) e^{o(1)},
$$


where $E_{\Phi}:=\varepsilon^{-\mathbf{i}(n-1) \sum_{j=1}^{n} v_{j}} \varepsilon^{-\frac{n(n-1)}{2}} e^{-\frac{n(n-1)}{2} \mathcal{A}(\varepsilon)}$, and from Proposition 6.5,

$$
P_{\lambda}\left(1 / z_{1}, \ldots, 1 / z_{n}\right)=\overline{E_{P}} \Psi_{-v_{1}, \ldots,-v_{n}}\left(x_{1}, \ldots, x_{n}\right) e^{o(1)},
$$

where $\overline{E_{P}}=\varepsilon^{-\frac{n(n-1)}{2}} \varepsilon^{\mathbf{i}(k+n-1) \sum_{j=1}^{n} v_{j}} e^{-\frac{n(n-1)}{2} \mathcal{A}(\varepsilon)}$. In all the above asymptotics, the $o$ (1) errors go to zero uniformly for $x \in \mathbb{K}$ and $v \in V$. Further, we have

$$
\mathfrak{m}_{n}^{q, 0}(z) \prod_{i=1}^{n} \frac{d z_{i}}{z_{i}}=E_{\mathfrak{m}} \mathfrak{m}_{n}(v) \prod_{i=1}^{n} d v_{i} e^{o(1)}, \quad E_{\mathfrak{m}}=\varepsilon^{n^{2}} e^{n(n-1) \mathcal{A}(\varepsilon)},
$$

where the error goes to zero uniformly for $v \in V$. The above asymptotics altogether suggest that uniformly for $x \in \mathbb{K}$,

$$
\left\langle\left\langle\Pi(z ; a, \gamma), P_{\lambda}(z)\right\rangle\right\rangle=E_{\Pi} E_{\Phi} \overline{E_{P}} E_{m} \mathcal{T}_{\alpha}^{\tau}(x) e^{o(1)},
$$

since integrands on both sides of (119) match when $\varepsilon \rightarrow 0$. However, the convergences above are valid for compact subsets of the integrand variable $v$. In order to justify that the integrand converges, one needs some tail decay estimate as $|v| \rightarrow \infty$, uniform with respect to $x \in \mathbb{K}$. For instance, it would be sufficient to prove that for

$$
V_{M}=\left\{z \in \mathbb{T}^{n}: z_{k}=e^{-\varepsilon a} e^{\mathbf{i} \varepsilon v_{k}} \text { and }\left|v_{k}\right|>M\right\},
$$

then

$$
\lim _{M \rightarrow \infty} \lim _{\varepsilon \rightarrow 0} \int_{z \in V_{M}} \frac{\Pi(z ; a, \gamma) \Phi(z) P_{\lambda}\left(z^{-1}\right) \mathfrak{m}_{n}^{q}(z)}{E_{\Pi} E_{\Phi} \overline{E_{P}} E_{\mathfrak{m}}} \prod_{i=1}^{n} \frac{d z_{i}}{z_{i}}=0
$$

uniformly for $x \in \mathbb{K}$. A similar estimate had already been proved in Step 4 of [BC14, Lemma 4.1.25]. One can estimate each of the quantities

$$
\left|\frac{\Pi(z ; a, \tau)}{E_{\Pi}}\right|, \quad\left|\frac{\Phi(z)}{E_{\Phi}}\right|, \quad\left|\frac{P_{\lambda}\left(z^{-1}\right)}{\overline{E_{P}}}\right| \text { and } \quad\left|\frac{\mathfrak{m}_{n}^{q}(z)}{E_{\mathfrak{m}}}\right| .
$$

While $\left|\frac{\Pi(z ; a, \tau)}{E_{\Pi}}\right|$ has a Gaussian decay in $v$, all other quantities have at most exponential growth (this is the reason why it is essential for us to keep a positive Plancherel specialization). Therefore, the integrand in (120) is bounded by a constant times $e^{-c \sum_{i=1}^{n} v_{i}^{2}}$, uniformly in $\varepsilon$ for $T \in D$. Thus, (120) is established. Finally, it is easy to check that $E_{\Pi} E_{\Phi} \overline{E_{P}} E_{m}$ matches the prefactor of $\mathcal{T}_{\alpha}^{\tau}(x)$ in the statement of Proposition 6.8. 
Definition 6.9. For $\alpha_{\circ}$ and $\alpha_{1}, \ldots, \alpha_{t} \in \mathbb{R}_{>0}, \tau>0$ and $1 \leqslant n \leqslant t$, we define the (ascending) half-space Whittaker process as a probability measure on $T:=\left(T_{j}^{(m)}\right)_{1 \leqslant j \leqslant m \leqslant n} \in \mathbb{R}^{\frac{n(n+1)}{2}}$ with density function given by

$\mathbb{P}(d T)$

$$
=\frac{\exp \left(\mathcal{F}_{\mathbf{i} \alpha_{1}, \ldots, \mathbf{i} \alpha_{n}}(T)\right) \mathcal{T}_{\alpha_{\circ}, \alpha_{n+1}, \ldots, \alpha_{t}}^{\tau}\left(T_{1}^{(n)}, \ldots, T_{n}^{(n)}\right)}{e^{\tau \sum_{i=1}^{n} \alpha_{i}^{2} / 2} \prod_{i=1}^{n} \Gamma\left(\alpha_{\circ}+\alpha_{i}\right) \prod_{j=n+1}^{t} \Gamma\left(\alpha_{j}+\alpha_{i}\right) \prod_{1 \leqslant i<j \leqslant n} \Gamma\left(\alpha_{j}+\alpha_{i}\right)} d T .
$$

Integrating the half-space Whittaker process over variables $T_{j}^{(m)}$ for $m<n$ defines the half-space Whittaker measure $\mathbb{P}_{\left(\alpha_{1}, \ldots, \alpha_{n}\right) ;\left(\alpha_{\circ}, \alpha_{n+1}, \ldots, \alpha_{t}\right), \tau}$ on $\left(T_{j}\right)_{1 \leqslant j \leqslant n} \in$ $\mathbb{R}^{n}$ with density

$$
\begin{aligned}
& \mathbb{P}_{\left(\alpha_{1}, \ldots, \alpha_{n}\right) ;\left(\alpha_{\circ}, \alpha_{n+1}, \ldots, \alpha_{t}\right), \tau}(d T) \\
& \quad=\frac{\psi_{\mathbf{i} \alpha_{1}, \ldots, \mathbf{i}_{n}}\left(T_{1}, \ldots, T_{n}\right) \mathcal{T}_{\alpha_{\circ}, \alpha_{n+1}, \ldots, \alpha_{t}}^{\tau}\left(T_{1}, \ldots, T_{n}\right)}{e^{\tau \sum_{i=1}^{n} \alpha_{i}^{2} / 2} \prod_{i=1}^{n} \Gamma\left(\alpha_{\circ}+\alpha_{i}\right) \prod_{j=n+1}^{t} \Gamma\left(\alpha_{j}+\alpha_{i}\right) \prod_{1 \leqslant i<j \leqslant n} \Gamma\left(\alpha_{j}+\alpha_{i}\right)} d T .
\end{aligned}
$$

The fact that these densities above define bona fide probability measures is not obvious. For $\alpha_{\circ} \in \mathbb{R}$ and $\alpha_{1}, \ldots, \alpha_{t} \in \mathbb{R}_{>0}$ with $\alpha_{\circ}+\alpha_{i}>0$, it follows from the limits of Propositions 6.5 and 6.8 that the density is nonnegative. The next proposition shows that the density integrates to 1 .

Proposition 6.10. Let $\tau>0, n \leqslant t$ and $\alpha_{1}, \ldots, \alpha_{t}>0$ such that $\alpha_{i}+\alpha_{\circ}>0$ for all $1 \leqslant i \leqslant n$. Then,

$$
\begin{aligned}
& \int_{\mathbb{R}^{n}} d x \psi_{\mathbf{i} \alpha_{1}, \ldots, \mathbf{i} \alpha_{n}}(x) \mathcal{T}_{\alpha_{\circ}, \alpha_{n+1}, \ldots, \alpha_{t}}^{\tau}(x) \\
& \quad=e^{\tau \sum_{i=1}^{n} \alpha_{i}^{2} / 2} \prod_{i=1}^{n} \Gamma\left(\alpha_{\circ}+\alpha_{i}\right) \prod_{j=n+1}^{t} \Gamma\left(\alpha_{j}+\alpha_{i}\right) \prod_{1 \leqslant i<j \leqslant n} \Gamma\left(\alpha_{i}+\alpha_{j}\right) .
\end{aligned}
$$

This is formally the limit of generalized Littlewood summation identity (28) via Propositions 6.5 and 6.8, though this does not constitute a proof because these limits hold only for $x$ in a compact set. However, we will see that the result can be deduced from the orthogonality of Whittaker functions along with certain bounds on the tails of the half-space Whittaker measure. We will first state these bounds and then prove Proposition 6.10.

We start with a general bound on the growth of Whittaker functions.

LEMMA 6.11. For any fixed $y \geqslant 0$, there exists a constant $C>0$ such that $\left|\psi_{\nu}(x)\right| \leqslant C e^{y \sum_{i=1}^{n}\left|x_{i}\right|}$ for all $\nu \in \mathbb{C}^{n}$ such that $\left|\mathfrak{I m}\left[v_{j}\right]\right| \leqslant y$, for all $1 \leqslant j \leqslant n$. 
In particular, if $\alpha \in \mathbb{C}^{n}$ is such that $c_{1}<\mathfrak{R e}\left[\alpha_{i}\right]<c_{2}$ for all $1 \leqslant i \leqslant n$,

$$
\left|\psi_{\mathbf{i} \alpha}(x)\right| \leqslant C e^{-c_{2} \sum_{i=1}^{n} x_{i}+\left(c_{2}-c_{1}\right) \sum_{i=1}^{n}\left|x_{i}\right|} .
$$

Proof. As [BC14, Lemma 4.1.19], the first statement follows by recurrence on $n$ using the recursive structure in Givental's integral representation (see [BC14, (4.2), (4.3)]). The second part of the statement comes from the shift property of Whittaker functions $\Psi_{v}(x)=\Psi_{v_{1}-\mathbf{i} c, \ldots, v_{n}-\mathbf{i c}}(x) e^{-c \sum_{i=1}^{n} x_{i}}$. Using the first part, we may write

$$
\left|\psi_{\mathbf{i} \alpha}(x)\right| \leqslant\left|\psi_{\mathbf{i} \alpha_{1}-\mathbf{i} c_{2}, \ldots, \mathbf{i} \alpha_{n}-\mathbf{i} c_{2}}(x) e^{-c_{2} \sum_{i=1}^{n} x_{i}}\right| \leqslant C e^{-c_{2} \sum_{i=1}^{n} x_{i}+\left(c_{2}-c_{1}\right) \sum_{i=1}^{n}\left|x_{i}\right|} .
$$

REMARK 6.12. In the Weyl chamber $\mathbb{W}_{n}:=\left\{x \in \mathbb{R}^{n}: x_{1} \geqslant x_{2} \geqslant \cdots \geqslant x_{n}\right\}$, one can refine the estimate from Lemma 6.11 (see for example [BO11, Corollary 2.3]). For any $n \geqslant 1$, there exists a constant $C>0$ such that for $x \in \mathbb{W}_{n}$ we have

$$
\left|\psi_{v}(x) e^{-\mathbf{i} \sum_{i=1}^{n} v_{i} x_{i}}\right| \leqslant C
$$

When the index of Whittaker functions belongs to $\mathbb{R}^{n}$, Whittaker functions have doubly exponential decay away from the Weyl chamber.

Proposition 6.13 [BC14, Proposition 4.1.3]. For $x \in \mathbb{R}^{n}$, define $\sigma(x)=\{i$ : $\left.x_{i}-x_{i+1} \leqslant 0\right\}$. For each $\sigma \subset\{1, \ldots, n\}$, there exists a polynomial $A_{\sigma}$ such that for all $\nu \in \mathbb{R}^{n}$ and for all $x \in \mathbb{R}^{n}$ with $\sigma(x)=\sigma$, we have

$$
\left|\psi_{\nu}(x)\right| \leqslant A_{\sigma}(x) \prod_{i \in \sigma} \exp \left(-e^{-\frac{x_{i}-x_{i+1}}{2}}\right) .
$$

This implies a similar bound for $\mathcal{T}^{\tau}(x)$.

Proposition 6.14. Fix $n \geqslant 1, k \geqslant 0, \tau>0$ and $\alpha_{1}, \ldots, \alpha_{k} \in \mathbb{R}$. Let $a>0$ be such that $a+\alpha_{i}>0$ for all $1 \leqslant i \leqslant k$. Then, there exists a polynomial $A$ such that for all $x \in \mathbb{R}^{n}$

$$
\left|\mathcal{T}_{\alpha_{1}, \ldots, \alpha_{k}}^{\tau}(x)\right| \leqslant A(x) e^{a \sum_{i=1}^{n} x_{i}} \prod_{i=1}^{n-1} \exp \left(-e^{-\frac{x_{i}-x_{i+1}}{2}}\right) .
$$

Proof. We may use the change of variables $v=\tilde{v}+\mathbf{i} a$ in (118) so that the integration is over $\mathbb{R}^{n}$ and use Proposition 6.13 to bound the Whittaker function inside the integral. 
Proposition 6.15. Fix $n \geqslant 1, k \geqslant 0, \tau>0$ and $\alpha_{1}, \ldots, \alpha_{k} \in \mathbb{R}$. Let $R>0$ be such that $R+\alpha_{i}>0$ for all $1 \leqslant i \leqslant k$. Then, there exists a constant $C>0$ such that for all $x \in \mathbb{R}^{n}$,

$$
\left|\mathcal{T}_{\alpha_{1}, \ldots, \alpha_{k}}^{\tau}(x)\right| \leqslant C e^{R \sum_{i=1}^{n} x_{i}} .
$$

Moreover, the function $x \mapsto e^{-R \sum_{i=1}^{n} x_{i}} \mathcal{T}_{\alpha_{1}, \ldots, \alpha_{k}}^{\tau}(x)$ is in $\mathbb{L}^{2}\left(\mathbb{R}^{n}\right)$.

Proof. Recall that in the definition of $\mathcal{T}_{\alpha_{1}, \ldots, \alpha_{k}}^{\tau}(x)$ in (118), the parameter $a>0$ can be taken arbitrarily as long as $a+\alpha_{i}>0$. Using the shift property of Whittaker functions $\psi_{\nu_{1}+\mathbf{i} a, \ldots, \nu_{n}+\mathbf{i} a}=e^{-a \sum_{i=1}^{n} x_{i}} \psi_{\nu_{1}, \ldots, \nu_{n}}(x)$ and the change of variables $v_{i}=\tilde{v}_{i}+\mathbf{i} a$ for all $1 \leqslant i \leqslant n$, we obtain

$$
\begin{aligned}
& \left|\mathcal{T}_{\alpha_{1}, \ldots, \alpha_{k}}^{\tau}(x)\right| \leqslant C e^{a \sum_{i=1}^{n} x_{i}} \int_{\mathbb{R}^{n}} \psi_{-\tilde{v}}(x) e^{-\tau \sum_{j=1}^{n} \tilde{v}_{j}^{2} / 2} \\
& \quad \times\left|\prod_{i=1}^{k} \prod_{j=1}^{n} \Gamma\left(\alpha_{i}-\mathbf{i} \tilde{v}_{j}+a\right) \prod_{1 \leqslant i<j \leqslant n} \Gamma\left(-\mathbf{i}\left(\tilde{v}_{i}+\tilde{v}_{j}\right)+2 a\right) \mathfrak{m}_{n}(v)\right| d \tilde{v} .
\end{aligned}
$$

Using Lemma 6.11, the integral can be bounded by a constant (depending on $a$ but not $x$ ), which yields the first part of the statement of Proposition 6.15 by choosing $a=R$. The fact that the integral above, seen as a function of $x$, is bounded in $\mathbb{L}^{2}\left(\mathbb{R}^{n}\right)$ follows from the orthogonality of Whittaker functions (105) and the Gaussian decay of the integrand.

The estimate from Proposition 6.15 will be useful in Section 6.7, but it is not sufficiently sharp for the proof of Proposition 6.10. The next proposition is a refinement.

Proposition 6.16. Fix $n \geqslant 2, k \geqslant 0, \tau>0, \alpha_{1}, \ldots, \alpha_{k} \in \mathbb{R}$. Let $a>0$ be such that $a+\alpha_{i}>0$ for all $1 \leqslant i \leqslant k$. For any $R>a$, there exists a polynomial $C(x)$ such that for $x \in \mathbb{R}^{n}$,

$$
\left|\mathcal{T}_{\alpha_{1}, \ldots, \alpha_{k}}^{\tau}(x)\right| \leqslant C(x) \prod_{i: x_{i}<0} e^{R x_{i}} \prod_{i: x_{i} \geqslant 0} e^{a x_{i}} .
$$

Proof. In the proof of Proposition 6.15, we have seen that the ability to freely shift the integration contours can be translated into a decay bound. In order to obtain a sharper bound, we need to shift each contour separately by a distance depending on the sign of $x_{i}$. Since we may not use the shift property of Whittaker functions anymore, it would be very convenient to decompose the Whittaker function $\psi_{v}(x)$ as linear combinations of $\prod_{i} e^{\mathbf{i} i_{i} x_{i}}$. Such a decomposition exists, in terms of the fundamental Whittaker functions introduced in [Has82]. We will 
be using notations from [O'C14, Section 8]. Using [O'C14, Proposition 3] (see also [O'C14, Corollary 3]), we may write

$$
\mathcal{T}_{\alpha}^{\tau}(x)=\int_{(\mathbb{R}+\mathbf{i} a)^{n}} m_{-\mathbf{i} v}(x) e^{-\tau \sum_{j=1}^{n} v_{j}^{2} / 2} Z(\alpha, v) \prod_{i<j}\left(\mathbf{i} v_{i}-\mathbf{i} v_{j}\right) d v,
$$

where we have used the shorthand notation

$$
Z(\alpha, v)=\prod_{i=1}^{k} \prod_{j=1}^{n} \Gamma\left(\alpha_{i}-\mathbf{i} v_{j}\right) \prod_{1 \leqslant i<j \leqslant n} \Gamma\left(-\mathbf{i}\left(v_{i}+v_{j}\right)\right),
$$

and $m_{-\mathbf{i} v}(x)$ are fundamental Whittaker functions defined as follows. For $n \geqslant$ 2 and $m \in\left(\mathbb{Z}_{\geqslant 0}\right)^{n-1}$, we define analytic coefficients $a_{n, m}(v)$ by the recurrence [IS07, Theorem 15]

$$
a_{2, m}(v)=\frac{1}{m ! \Gamma\left(v_{1}-v_{2}+m+1\right)},
$$

and for $n>2$,

$$
a_{n, m}(v)=\sum_{k \in(\mathbb{Z} \geqslant 0)^{n-2}} a_{n-1, k}(\mu) \prod_{i=1}^{n-1} \frac{1}{\left(m_{i}-k_{i}\right) !} \frac{1}{\Gamma\left(v_{i}-v_{n}+m_{i}-k_{i-1}\right)},
$$

where the sum runs over $k \in\left(\mathbb{Z}_{\geqslant 0}\right)^{n-2}$ such that $k_{i} \leqslant m_{i}$, we adopt the convention that $k_{0}=k_{n-1}=0$, and $\mu=\left(v_{1}+v_{n} /(n-1), \ldots, v_{n-1}+v_{n} /(n-1), v_{n}\right)$. Then, fundamental Whittaker functions are defined by the series

$$
m_{v}(x)=\sum_{m \in\left(\mathbb{Z}_{\geqslant 0}\right)^{n-1}} a_{n, m}(v) \exp \left(-\sum_{i=1}^{n-1} m_{i}\left(x_{i}-x_{i+1}\right)+\sum_{i=1}^{n} v_{i} x_{i}\right),
$$

which is absolutely convergent [Has82, Lemma 4.6]. We will need the following estimate.

LEMMA 6.17. Fix $a, R>0$ and $n \geqslant 2$. Let us define

$$
A_{N}=\max _{\substack{m \in(\mathbb{Z} \geqslant 0)^{n-1} \\ \sum_{i=1}^{n-1} m_{i}=N}}\left\{\left|a_{n, m}(\mathbf{i} v)\right|\right\} .
$$

There exist positive constants $C, c, c^{\prime}$ such that for $v \in \mathbb{C}^{n}$ where for all $i, v_{i} \in$ $(\mathbb{R}+\mathbf{i} a)^{n}$ or $v_{i} \in(\mathbb{R}+\mathbf{i}(a+R))^{n}$, we have

$$
A_{N} \leqslant c e^{c^{\prime} \sum_{i=1}^{n}\left|\mathfrak{R}_{\mathfrak{e}}\left[v_{i}\right]\right|} \frac{C^{N}}{(N !)^{2}} .
$$


Proof. We adapt the proof of [Has82, Lemma 4.5], which concerns the case where $v$ is restricted to a compact set. The coefficients $a_{n, m}(v)$ satisfy another recurrence [IS07, Theorem 15]:

$$
\left(\sum_{i=1}^{n-1} m_{i}^{2}-\sum_{i=1}^{n-2} m_{i} m_{i+1}+\sum_{i=1}^{n-1}\left(v_{i}-v_{i+1}\right) m_{i}\right) a_{n, m}(\nu)=\sum_{i=1}^{n-1} a_{n, m-e_{i}}(v),
$$

where $m-e_{i}=\left(m_{1}, \ldots, m_{i}-1, \ldots, m_{n-1}\right)$, with $a_{n, m}=0$ if $m \notin\left(\mathbb{Z}_{\geqslant 0}\right)^{n-1}$ and

$$
a_{n, 0}(v)=\prod_{i<j} \frac{1}{\Gamma\left(v_{i}-v_{j}+1\right)} .
$$

We have

$\sum_{i=1}^{n-1} m_{i}^{2}-\sum_{i=1}^{n-2} m_{i} m_{i+1}+\sum_{i=1}^{n-1}\left(\mathbf{i} v_{i}-\mathbf{i} v_{i+1}\right) m_{i}=\sum_{i=0}^{n-1} \frac{1}{2}\left(m_{i}-m_{i+1}\right)^{2}+\sum_{i=1}^{n-1}\left(\mathbf{i} v_{i}-\mathbf{i} v_{i+1}\right) m_{i}$,

with the convention that $m_{0}=m_{n}=0$. Note that if $N=m_{1}+\cdots+m_{n-1}$, we may write $N=\sum_{i=0}^{n-1}\left(m_{i}-m_{i+1}\right)(i+1)$ so that using the Cauchy-Schwartz inequality,

$$
N^{2} \leqslant \sum_{i=0}^{n-1}\left(m_{i}-m_{i+1}\right)^{2} \sum_{i=1}^{n} i^{2} \leqslant n^{3} \sum_{i=0}^{n-1}\left(m_{i}-m_{i+1}\right)^{2} .
$$

Then, using $|z| \geqslant|\mathfrak{R e}[z]|$, we can write

$$
\left|\sum_{i=1}^{n-1} m_{i}^{2}-\sum_{i=1}^{n-2} m_{i} m_{i+1}+\sum_{i=1}^{n-1}\left(\mathbf{i} v_{i}-\mathbf{i} v_{i+1}\right) m_{i}\right|>\frac{1}{2 n^{3}} N^{2}-N R,
$$

where $N=m_{1}+\cdots+m_{n-1}$. For $N>4 R n^{3}$, we have $N^{2}-N R>N^{2} /\left(4 n^{3}\right)$. In (126), we may bound each term in the sum on the right-hand side by $A_{N-1}$ so that for $N>4 R n^{3}$,

$$
A_{N} \leqslant \frac{4 n^{4}}{N^{2}} A_{N-1}
$$

Thus, if (125) is true for $N \leqslant 4 R n^{3}$, then it is true for all $N$, by choosing $C$ in (125) larger than $4 n^{4}$. Since $R$ and $n$ are fixed, we may bound $A_{N}$ for all $N \leqslant 4 R n$ by $c e^{c^{\prime} \sum_{i=1}^{n} \mathfrak{R e}\left[v_{i}\right]}$ for some constants $c, c^{\prime}$. Indeed, using the recurrence formula (124), coefficients $a_{n, m}$ are finite sums of finite products of Gamma factors, which can be bounded by $c e^{c^{\prime} \sum_{i=1}^{n}\left|\mathfrak{R e}\left[v_{i}\right]\right|}$. 
Going back to the proof of Proposition 6.15, we can rewrite (123) as

$$
\begin{aligned}
\mathcal{T}_{\alpha}^{\tau}(x)= & \left.\sum_{m \in(\mathbb{Z} \geqslant 0)^{n-1}} \int_{(\mathbb{R}+\mathbf{i} i)^{n}} a_{n, m}(\mathbf{i} v) \exp \left(-\sum_{i=1}^{n-1} m_{i}\left(x_{i}-x_{i+1}\right)-\sum_{i=1}^{n} \mathbf{i} v_{i} x_{i}\right)\right) \\
& \times e^{-\tau \sum_{j=1}^{n} v_{j}^{2} / 2} Z(\alpha, v) \prod_{i<j}\left(\mathbf{i} v_{i}-\mathbf{i} v_{j}\right) d \nu .
\end{aligned}
$$

For all $i$, if $x_{i}<0$, we shift the contour by $\mathbf{i}(R-a)$ (that is, from $\mathbb{R}+\mathbf{i} a$ to $\mathbb{R}+\mathbf{i} R)$ and subsequently use the change of variables $v_{i}=\tilde{v}_{i}+\mathbf{i} R$ so that $\tilde{v}_{i} \in \mathbb{R}$. If $x_{i} \geqslant 0$, we use the change of variables $v_{i}=\tilde{v}_{i}+\mathbf{i} a$ so that $\tilde{v}_{i} \in \mathbb{R}$. The factor $\exp \left(-\sum_{i=1}^{n} \mathbf{i} \tilde{v}_{i} x_{i}\right)$ can be simply bounded by 1 . We obtain that $\mathcal{T}_{\alpha}^{\tau}(x)$ is bounded by the product of three terms:

- $\prod_{i: x_{i}<0} e^{R x_{i}} \prod_{i: x_{i} \geqslant 0} e^{a x_{i}}$;

- an absolutely convergent integral over $\tilde{v} \in \mathbb{R}$, which does not depend on $x$; and

- the series

$$
\sum_{m \in\left(\mathbb{Z}_{\geqslant 0}\right)^{n-1}} \frac{C^{m_{1}+\cdots+m_{n-1}} \exp \left(-\sum_{i=1}^{n-1} m_{i}\left(x_{i}-x_{i+1}\right)\right)}{\left(\left(m_{1}+\ldots m_{n-1}\right) !\right)^{2}},
$$

where $C$ is the constant in Lemma 6.17.

Using that $\left(m_{1}+\cdots+m_{n-1}\right) ! \geqslant m_{1} ! \ldots m_{n-1} !$ and the inequality $\sum_{k=0}^{\infty} \frac{x^{k}}{(k !)^{2}} \leqslant$ $\exp (2 \sqrt{x})$ for positive $x$, we may bound the series as

$$
\sum_{m \in\left(\mathbb{Z}_{\geqslant 0}\right)^{n-1}} \frac{C^{m_{1}+\cdots+m_{n-1}} \exp \left(-\sum_{i=1}^{n-1} m_{i}\left(x_{i}-x_{i+1}\right)\right)}{\left(\left(m_{1}+\ldots m_{n-1}\right) !\right)^{2}} \leqslant \prod_{i=1}^{n-1} e^{2 C e^{\frac{-\left(x_{i}-x_{i+1}\right)}{2}}} .
$$

Thus we have arrived at

$$
\left|\mathcal{T}_{\alpha_{1}, \ldots, \alpha_{k}}^{\tau}(x)\right| \leqslant C^{\prime} \prod_{i: x_{i}<0} e^{R x_{i}} \prod_{i: x_{i} \geqslant 0} e^{a x_{i}} \prod_{i=1}^{n-1} e^{2 C e^{\frac{-\left(x_{i}-x_{i+1}\right)}{2}}} .
$$

Now we may combine this result with the bound of Proposition 6.14. Let us write

$$
\left|\mathcal{T}_{\alpha_{1}, \ldots, \alpha_{k}}^{\tau}(x)\right|^{2 C+1}=\left|\mathcal{T}_{\alpha_{1}, \ldots, \alpha_{k}}^{\tau}(x)\right|^{2 C}\left|\mathcal{T}_{\alpha_{1}, \ldots, \alpha_{k}}^{\tau}(x)\right|,
$$

where $C$ is the same constant as above. We bound the first factor with Proposition 6.14 and the second factor with (128). The doubly exponential factors cancel, and this concludes the proof of the proposition. 
Proof of Proposition 6.10. Let

$$
f\left(\alpha_{1}, \ldots, \alpha_{n}\right)=\int_{\mathbb{R}^{n}} d x \psi_{\mathbf{i} \alpha_{1}, \ldots, \mathbf{i} \alpha_{n}}(x) \mathcal{T}_{\alpha_{o}, \alpha_{n+1}, \ldots, \alpha_{t}}^{\tau}(x) .
$$

Recall the definition of the function $\mathcal{T}^{\tau}$ in (118). Using orthogonality of Whittaker functions, we deduce that when $\alpha_{1}, \ldots, \alpha_{n}$ belong to $\mathbf{i} \mathbb{R}+a$ (where $a$ is the real part of the contour in (118)),

$$
f\left(\alpha_{1}, \ldots, \alpha_{n}\right)=e^{\tau \sum_{i=1}^{n} \alpha_{i}^{2} / 2} \prod_{i=1}^{n} \Gamma\left(\alpha_{\circ}+\alpha_{i}\right) \prod_{j=n+1}^{t} \Gamma\left(\alpha_{j}+\alpha_{i}\right) \prod_{1 \leqslant i<j \leqslant n} \Gamma\left(\alpha_{i}+\alpha_{j}\right) .
$$

Recall that Whittaker functions are analytic functions in their index. We wish to analytically continue the above identity for all $\alpha$ such that $\mathfrak{R e}\left[\alpha_{i}\right] \geqslant a$, for some $a>0$ such that $a>-\alpha_{\circ}$. As in the proof of [BC14, Proposition 4.1.18], we prove that $f\left(\alpha_{1}, \ldots, \alpha_{n}\right)$ is analytic by showing that for any compact region $A \subset\left\{z \in \mathbb{C}^{n}: \mathfrak{R e}\left[z_{i}\right]>a\right\}$ and any $\varepsilon>0$, there exists a compact subset $\mathbb{K} \subset \mathbb{R}^{n}$ such that for all $\alpha \in A$,

$$
\int_{\mathbb{R}^{n} \backslash \mathbb{K}} d x\left|\psi_{\mathbf{i} \alpha_{1}, \ldots, \mathbf{i} \alpha_{n}}(x) \mathcal{T}_{\alpha_{o}, \alpha_{n+1}, \ldots, \alpha_{t}}^{\tau}(x)\right|<\varepsilon .
$$

Consider a compact set $A \subset \mathbb{C}^{n}$ such that for all $z \in A, \mathfrak{R e}\left[z_{i}\right]>a$. Let $\underline{a}>0$ be such that $\max \left\{0,-\alpha_{\circ}\right\}<\underline{a}<a$ and let $\bar{a}$ be such that $\mathfrak{R e}\left[z_{i}\right]<\bar{a}$ for $\alpha \in A$. On the one hand, Proposition 6.16 shows that for any $R>0$, there exists a polynomial $C(x)$ such that

$$
\left|\mathcal{T}_{\alpha_{o}, \alpha_{n+1}, \ldots, \alpha_{t}}^{\tau}(x)\right| \leqslant C(x) e^{\underline{\underline{a}} \sum_{i=1}^{n} x_{i}} \prod_{i: x_{i}<0} e^{R x_{i}} .
$$

On the other hand, Lemma 6.11 shows that if $a<\mathfrak{R e}\left[\alpha_{i}\right]<\bar{a}$,

$$
\left|\psi_{\mathbf{i} \alpha}(x)\right| \leqslant C e^{-\bar{a} \sum_{i=1}^{n} x_{i}+(\bar{a}-a) \sum_{i=1}^{n}\left|x_{i}\right|} .
$$

Combining these two bounds and using the fact that for $x<0,2 x=x-|x|$, we obtain that for any $R>0$,

$$
\begin{aligned}
\left|\psi_{\mathbf{i} \alpha}(x) \mathcal{T}_{\alpha_{0}, \alpha_{n+1}, \ldots, \alpha_{t}}^{\tau}(x)\right| & \leqslant C(x) e^{-(a-\underline{a}) \sum_{i=1}^{n} x_{i}} e^{(\bar{a}-a) \sum_{i=1}^{n}\left|x_{i}\right|-x_{i}} \prod_{i: x_{i}<0} e^{R x_{i}}, \\
& \leqslant C(x) e^{-(a-\underline{a}) \sum_{i=1}^{n} x_{i}} e^{(\bar{a}-a-R / 2) \sum_{i=1}^{n}\left|x_{i}\right|-x_{i}} .
\end{aligned}
$$

Let $\mathbb{A}^{(S)}=\left\{x \in \mathbb{R}^{n}: \sum_{i=1}^{n} x_{i} \leqslant S\right\}$. We have shown that there exist positive constants $C, c$ such that when $x \notin\left(\mathbb{R}_{>-M}\right)^{n} \cap \mathbb{A}^{(S)}$,

$$
\left|\psi_{\mathbf{i} \alpha}(x) \mathcal{T}_{\alpha_{o}, \alpha_{n+1}, \ldots, \alpha_{t}}^{\tau}(x)\right| \leqslant C e^{-c S}+C e^{-c M} .
$$


Thus for any $\varepsilon>0$, taking the compact set $\mathbb{K}$ on the left-hand side of (129) as $\mathbb{K}=\left(\mathbb{R}_{>-M}\right)^{n} \cap \mathbb{A}^{(S)}$ for $M, S$ large enough, (129) holds. This concludes the proof of Proposition 6.10.

Proposition 6.18. Consider scalings (111) and (112). Assume that $\tau>0$ and $\alpha_{1}, \ldots, \alpha_{t}>0$ are such that $\alpha_{i}+\alpha_{\circ}>0$ for all $1 \leqslant i \leqslant n$. Then the half-space $q$-Whittaker measure $\mathbb{P}_{\left(a_{1}, \ldots, a_{n}\right),\left(a_{0}, a_{n+1}, \ldots, a_{t}\right), \gamma}^{q}$ weakly converges to the half-space Whittaker measure $\mathbb{P}_{\left(\alpha_{1}, \ldots, \alpha_{n}\right),\left(\alpha_{0}, \alpha_{n+1}, \ldots, \alpha_{t}\right), \tau}$.

Proof. Limits (116) and (117) and Propositions 6.5 and 6.8 show that the density of the half-space $q$-Whittaker measure converges to the density of the half-space Whittaker measure for $x$ in a compact subset of $\mathbb{R}^{n}$. Proposition 6.10 shows that the half-space Whittaker measure is a probability measure. This is sufficient to deduce the weak convergence.

REMARK 6.19. More generally, the half-space $q$-Whittaker process also converges to the half-space Whittaker process.

6.3. Observables and integral formulas. Define the functions

$$
\begin{aligned}
& \mathcal{G}(v)=\frac{e^{-\tau v^{2} / 2}}{\Gamma\left(\alpha_{\circ}+v\right)} \frac{\prod_{j=1}^{n} \Gamma\left(v-\alpha_{j}\right)}{\prod_{j=1}^{t} \Gamma\left(\alpha_{j}+v\right)} \text { and } \\
& \overline{\mathcal{G}}(v)=\frac{e^{-\tau v^{2} / 2}}{\Gamma\left(\alpha_{\circ}+v\right)} \frac{\prod_{j=1}^{n} \Gamma\left(\alpha_{j}-v\right)}{\prod_{j=1}^{t} \Gamma\left(\alpha_{j}+v\right)} .
\end{aligned}
$$

\subsubsection{Laplace transforms}

COROllary 6.20. Let $t \geqslant n \geqslant 1$, and $\tau>0$. Assume the following:

(i) The parameters $\alpha_{1}, \ldots, \alpha_{t}>0, \alpha_{\circ} \in \mathbb{R}$ are chosen so that for all $1 \leqslant i$, $j \leqslant n, \alpha_{i}-\alpha_{j}<\min _{1 \leqslant i \leqslant n}\left\{\alpha_{i}\right\}, \alpha_{i}-\alpha_{j}<1$ and $\alpha_{i}+\alpha_{\circ}>0$.

(ii) $R \in(0,1)$ is chosen so that $R<\min _{1 \leqslant i \leqslant n}\left\{\alpha_{i}, \alpha_{\circ}+\alpha_{i}\right\}$ and $R>$ $\max _{1 \leqslant i, j \leqslant n}\left\{\alpha_{i}-\alpha_{j}\right\}$.

Then, under the Whittaker measure $\mathbb{P}_{\left(\alpha_{1}, \ldots, \alpha_{n}\right),\left(\alpha_{0}, \alpha_{n+1}, \ldots, \alpha_{t}, \tau\right)}$,

$$
\mathbb{E}\left[e^{u e^{T_{1}}}\right]=\sum_{k=0}^{n} \frac{1}{k !} \int_{\mathcal{D}_{R}} \frac{d s_{1}}{2 \mathbf{i} \pi} \cdots \int_{\mathcal{D}_{R}} \frac{d s_{k}}{2 \mathbf{i} \pi} \oint \frac{d v_{1}}{2 \mathbf{i} \pi} \cdots \oint \frac{d v_{k}}{2 \mathbf{i} \pi}
$$




$$
\begin{aligned}
& \times \prod_{1 \leqslant i<j \leqslant k} \frac{\left(s_{i}+v_{j}-s_{j}-v_{i}\right)\left(v_{i}-v_{j}\right) \Gamma\left(v_{i}+v_{j}\right) \Gamma\left(v_{i}+v_{j}-s_{i}-s_{j}\right)}{\left(v_{j}-s_{j}-v_{i}\right)\left(v_{i}-s_{i}-v_{j}\right) \Gamma\left(v_{i}+v_{j}-s_{i}\right) \Gamma\left(v_{i}+v_{j}-s_{j}\right)} \\
& \times \prod_{i=1}^{k}\left[\Gamma\left(-s_{i}\right) \Gamma\left(1+s_{i}\right) \frac{\overline{\mathcal{G}}\left(v_{i}\right)}{\overline{\mathcal{G}}\left(v_{i}-s_{i}\right)} \frac{\Gamma\left(2 v_{i}\right)}{\Gamma\left(2 v_{i}-s_{i}\right)} \frac{(-u)^{s_{i}}}{s_{i}}\right],
\end{aligned}
$$

where the contours for each variable $v_{i}$ are positively oriented circles enclosing the poles $\left\{\alpha_{j}\right\}_{1 \leqslant j \leqslant n}$, and no other singularity of the integrand, and $\mathcal{D}_{R}=R+\mathbf{i} \mathbb{R}$ oriented upwards as before.

It should be noted that the assumption that the Plancherel component $\tau$ is positive is essential here. The right-hand side of (131) would simply diverge for $\tau=0$.

Proof. This is the $q \rightarrow 1$ limit of Corollary 4.7. Consider scalings (111) and (112). Recall the $q$-Gamma and the $q$-exponential functions from Section 2.1. Setting $z=u(1-q)^{t+n} \exp \left(-\varepsilon^{-1} \tau\right)$ in Corollary 4.7 and using the change of variables $w_{i}=q^{v_{i}}$ yields

$$
\begin{aligned}
& \mathbb{E}\left[e_{q}\left(u e^{T_{1}(q)}\right)\right]=\sum_{k=0}^{n} \frac{1}{k !} \int_{\mathcal{D}_{R}} \frac{d s_{1}}{2 \mathbf{i} \pi} \cdots \int_{\mathcal{D}_{R}} \frac{d s_{k}}{2 \mathbf{i} \pi} \oint \frac{d v_{1}}{2 \mathbf{i} \pi} \cdots \oint \frac{d v_{k}}{2 \mathbf{i} \pi} \\
& \times \prod_{1 \leqslant i<j \leqslant k} \frac{\left(s_{i}+v_{j}-s_{j}-v_{i}\right)\left(v_{i}-v_{j}\right) \Gamma_{q}\left(v_{i}+v_{j}\right) \Gamma_{q}\left(v_{i}+v_{j}-s_{i}-s_{j}\right)}{\left(v_{j}-s_{j}-v_{i}\right)\left(v_{i}-s_{i}-v_{j}\right) \Gamma_{q}\left(v_{i}+v_{j}-s_{i}\right) \Gamma_{q}\left(v_{i}+v_{j}-s_{j}\right)} \\
& \times \prod_{i=1}^{k}\left[\Gamma\left(-s_{i}\right) \Gamma\left(1+s_{i}\right) \prod_{j=1}^{n}\left(\frac{\Gamma_{q}\left(\alpha_{j}-v_{i}\right) \Gamma_{q}\left(\alpha_{j}+v_{i}-s_{i}\right)}{\Gamma_{q}\left(\alpha_{j}+v_{i}\right) \Gamma_{q}\left(\alpha_{j}+s_{i}-v_{i}\right)}\right) \frac{\Gamma_{q}\left(2 v_{i}\right)}{\Gamma_{q}\left(2 v_{i}-s_{i}\right)}\right. \\
& \left.\times \frac{\Gamma_{q}\left(\alpha_{\circ}+v_{i}-s_{i}\right)}{\Gamma_{q}\left(\alpha_{\circ}+v_{i}\right)} \prod_{j=n+1}^{t}\left(\frac{\Gamma_{q}\left(\alpha_{j}+v_{i}-s_{i}\right)}{\Gamma_{q}\left(\alpha_{j}+v_{i}\right)}\right) \frac{(-u)^{s_{i}} e^{-\tau \varepsilon^{-1} s} e^{\gamma q^{v_{-}}\left(q^{-s_{i}}-1\right)}}{\left(1-q^{s_{i}}\right) q^{v_{i}}}\right] .
\end{aligned}
$$

Indeed, consider first the limit of the left-hand side. Using Proposition 6.18 and the convergence of the $q$-exponential function to the exponential on compact sets, $\mathbb{E}\left[e_{q}\left(u e^{T_{1}(q)}\right)\right]$ converges to the left-hand side of (131). Now we consider the right-hand side. Note that for any $x \in \mathbb{C} \backslash \mathbb{R}_{<0}, \Gamma_{q}(x) \underset{q \rightarrow 1}{\longrightarrow} \Gamma(x)$, and with $\gamma=\tau \varepsilon^{-2}$ and $w=q^{v}$, we have

$$
e^{-\tau \varepsilon^{-1} s} e^{\gamma\left(q^{-s} w-w\right)} \underset{\varepsilon \rightarrow 0}{\longrightarrow} e^{-\tau v s+\tau s^{2} / 2}=e^{\tau(v-s)^{2} / 2-\tau v^{2} / 2} .
$$

This shows that the integrand on the right-hand side in (132) converges pointwise to the integrand on the right-hand side of (131). To conclude using dominated 
convergence, we need to show that the integrands are uniformly (with respect to $q$ ) integrable. We may first evaluate the integrals over $v_{i}$. The residues occur when $z_{i}=\alpha_{p(i)}$ for some choice of $p:\{1, \ldots, k\} \rightarrow\{1, \ldots, n\}$. Hence,

$$
\begin{aligned}
\mathbb{E}\left[e_{q}\left(u e^{T_{1}(q)}\right)\right] & \\
= & \sum_{k=0}^{n} \frac{1}{k !} \int_{\mathcal{D}_{R}} \frac{d s_{1}}{2 \mathbf{i} \pi} \cdots \int_{\mathcal{D}_{R}} \frac{d s_{k}}{2 \mathbf{i} \pi} \\
& \times \sum_{p:\{1, \ldots, k\} \rightarrow\{1, \ldots, n\}} \prod_{1 \leqslant i<j \leqslant k}\left(\frac{\left(s_{i}+\alpha_{p(j)}-s_{j}-\alpha_{p(i)}\right)\left(\alpha_{p(i)}-\alpha_{p(j)}\right)}{\left(\alpha_{p(j)}-s_{j}-\alpha_{p(i)}\right)\left(\alpha_{p(i)}-s_{i}-\alpha_{p(j)}\right)}\right) \\
& \times \prod_{1 \leqslant i<j \leqslant k}\left(\frac{\Gamma_{q}\left(\alpha_{p(i)}+\alpha_{p(j)}\right) \Gamma_{q}\left(\alpha_{p(i)}+\alpha_{p(j)}-s_{i}-s_{j}\right)}{\Gamma_{q}\left(\alpha_{p(i)}+\alpha_{p(j)}-s_{i}\right) \Gamma_{q}\left(\alpha_{p(i)}+\alpha_{p(j)}-s_{j}\right)}\right) \\
& \times \prod_{i=1}^{k}\left[\Gamma\left(-s_{i}\right) \Gamma\left(1+s_{i}\right) \prod_{j=1}^{n}\left(\frac{\operatorname{Res}_{z=0}\left\{\Gamma_{q}(z)\right\} \Gamma_{q}\left(\alpha_{j}+\alpha_{p(i)}-s_{i}\right)}{\Gamma_{q}\left(\alpha_{j}+\alpha_{p(i)}\right) \Gamma_{q}\left(\alpha_{j}+s_{i}-\alpha_{p(i)}\right)}\right)\right. \\
& \times \frac{\Gamma_{q}\left(2 \alpha_{p(i)}\right)}{\Gamma_{q}\left(2 \alpha_{p(i)}-s_{i}\right)} \frac{\Gamma_{q}\left(\alpha_{\circ}+\alpha_{p(i)}-s_{i}\right)}{\Gamma_{q}\left(\alpha_{\circ}+\alpha_{p(i)}\right)} \\
& \left.\times \prod_{j=n+1}^{t}\left(\frac{\Gamma_{q}\left(\alpha_{j}+\alpha_{p(i)}-s_{i}\right)}{\Gamma_{q}\left(\alpha_{j}+\alpha_{p(i)}\right)}\right) \frac{(-u)^{s_{i}} e^{-\tau \varepsilon^{-1} s} e^{\gamma q^{-s}\left(q^{\alpha_{p(i)}}-1\right)}}{\left(1-q^{s_{i}}\right) q^{\alpha_{p(i)}}}\right] .
\end{aligned}
$$

For any fixed $a, b>0$, there exists a constant $C_{1}>0$ such that for any $y \in \mathbb{R}$ and $q \in\left(\frac{1}{2}, 1\right)$, we have (see [BC17, Lemma 2.7])

$$
\left|\frac{\Gamma_{q}(a+i y)}{\Gamma_{q}(b+i y)}\right| \leqslant C_{1}\left(|y|^{|b-a|+1}+1\right) .
$$

Moreover (see Lemma 5.11 in [BCFV15]), there exist constants $C_{2}, C_{3}$ such that for $x \in(a, b)$ and $y \in \mathbb{R}$,

$$
\left|\Gamma_{q}(x+\mathbf{i} y)\right|<C_{2}, \quad\left|\frac{1}{\Gamma_{q}(x+\mathbf{i} y)}\right|<e^{C_{3}|y|} .
$$

By a Taylor approximation in $\varepsilon$, for $\varepsilon$ small enough, there exists a constant $C_{4}$ such that

$$
\left|e^{-\tau \varepsilon^{-1} s} e^{\gamma q^{\alpha_{i}}\left(q^{-s}-1\right)}\right|<e^{-C_{4}|s|^{2}}, \quad s \in \mathcal{D}_{R} .
$$

Using these estimates, each integrand in (133) can be bounded uniformly in $q \in$ $(1 / 2,1)$ by

$$
\prod_{i=1}^{k} C_{5} e^{C_{6}\left|s_{i}\right|-C_{7}\left|s_{i}\right|^{2}}
$$


for some constants $C_{5}, C_{6}, C_{7}>0$, which is integrable over $\vec{s} \in\left(\mathcal{D}_{R}\right)^{k}$. Finally, the sum over $k$ is finite so that dominated convergence can be applied.

COROLlaRY 6.21. Let $t \geqslant n \geqslant 1, \alpha_{1}, \ldots, \alpha_{t}>0, \alpha_{\circ} \in \mathbb{R}$ such that $\alpha_{i}+\alpha_{\circ}>0$ and $\tau>0$. Assume that the parameters $\alpha_{1}, \ldots, \alpha_{n}$ are chosen such that for all $1 \leqslant i, j \leqslant n, \alpha_{i}-\alpha_{j}<1$, and let $R \in(0,1)$ be such that $R>\alpha_{i}-\alpha_{j}$ for all $1 \leqslant i, j \leqslant n$. Under the Whittaker measure $\mathbb{P}_{\left(\alpha_{1}, \ldots, \alpha_{n}\right),\left(\alpha_{0}, \alpha_{n+1}, \ldots, \alpha_{t}, \tau\right)}$,

$$
\begin{aligned}
\mathbb{E} & {\left[e^{u e^{-T_{n}}}\right]=\sum_{k=0}^{n} \frac{1}{k !} \int_{\mathcal{D}_{R}} \frac{d s_{1}}{2 \mathbf{i} \pi} \cdots \int_{\mathcal{D}_{R}} \frac{d s_{k}}{2 \mathbf{i} \pi} \oint \frac{d v_{1}}{2 \mathbf{i} \pi} \cdots \oint \frac{d v_{k}}{2 \mathbf{i} \pi} } \\
& \times \prod_{1 \leqslant i<j \leqslant k} \frac{\left(s_{j}+v_{j}-s_{i}-v_{i}\right)\left(v_{i}-v_{j}\right) \Gamma\left(v_{i}+v_{j}\right) \Gamma\left(s_{i}+s_{j}+v_{i}+v_{j}\right)}{\left(s_{i}+v_{i}-v_{j}\right)\left(s_{j}+v_{j}-v_{i}\right) \Gamma\left(s_{i}+v_{i}+v_{j}\right) \Gamma\left(s_{j}+v_{i}+v_{j}\right)} \\
& \times \prod_{i=1}^{k}\left[\Gamma\left(-s_{i}\right) \Gamma\left(1+s_{i}\right) \frac{\mathcal{G}\left(v_{i}\right)}{\mathcal{G}\left(v_{i}+s_{i}\right)} \frac{\Gamma\left(2 v_{i}\right)}{\Gamma\left(s_{i}+2 v_{i}\right)} \frac{(-u)^{s_{i}}}{-s_{i}}\right]
\end{aligned}
$$

where the contours for each variable $v_{i}$ are positively oriented circles enclosing the poles $\left\{\alpha_{j}\right\}_{1 \leqslant j \leqslant n}$, and no other singularity of the integrand.

Proof. The formula can be obtained from Corollary 4.3 similarly to the proof of Corollary 6.20.

\subsubsection{Moment formulas}

Proposition 6.22. Let $t \geqslant n \geqslant 1, \alpha_{1}, \ldots, \alpha_{t}>0$ and $\alpha_{\circ}>0$ so that $\alpha_{i}+\alpha_{\circ}>$ 0 for all $1 \leqslant i \leqslant n$. Under the Whittaker measure $\mathbb{P}_{\left(\alpha_{1}, \ldots, \alpha_{n}\right),\left(\alpha_{0}, \alpha_{n+1}, \ldots, \alpha_{t}, \tau\right)}$, we have the following moment formulas. For any $k \in \mathbb{Z}_{>0}$,

$$
\begin{aligned}
\mathbb{E}\left[e^{-k T_{n}}\right]= & e^{k \tau / 2} \oint \frac{d w_{1}}{2 \mathbf{i} \pi} \cdots \oint \frac{d w_{k}}{2 \mathbf{i} \pi} \prod_{1 \leqslant a<b \leqslant k} \frac{w_{a}-w_{b}}{w_{a}-w_{b}-1} \frac{1+w_{a}+w_{b}}{w_{a}+w_{b}} \\
& \times \prod_{m=1}^{k} \frac{\mathcal{G}\left(w_{m}\right)}{\mathcal{G}\left(w_{m}+1\right)} \frac{1}{2 w_{m}},
\end{aligned}
$$

where the positively oriented contours are such that for all $1 \leqslant c \leqslant k$, the contour for $w_{c}$ encloses $\left\{\alpha_{j}\right\}_{1 \leqslant j \leqslant n}$ and $\left\{w_{c+1}+1, \ldots, w_{k}+1\right\}$, and excludes the pole of the integrand at 0 .

For all $k \in \mathbb{Z}_{>0}$ such that $k<2 \min \left\{\alpha_{i}\right\}$ and $k<\alpha_{\circ}+\min \left\{\alpha_{i}\right\}$,

$$
\mathbb{E}\left[e^{k T_{1}}\right]={ }^{k \tau / 2} \oint \frac{d w_{1}}{2 \mathbf{i} \pi} \cdots \oint \frac{d w_{k}}{2 \mathbf{i} \pi} \prod_{1 \leqslant a<b \leqslant k} \frac{w_{a}-w_{b}}{w_{a}-w_{b}-1} \frac{1+w_{a}+w_{b}}{2+w_{a}+w_{b}}
$$




$$
\times \prod_{m=1}^{k} \frac{\overline{\mathcal{G}}(-w)}{\overline{\mathcal{G}}(-w-1)}\left(1+2 w_{m}\right)
$$

where the contours are such that for all $1 \leqslant c \leqslant k$, the contour for $w_{c}$ encloses $\left\{-\alpha_{j}\right\}_{1 \leqslant j \leqslant n}$ and $\left\{w_{c+1}+1, \ldots, w_{k}+1\right\}$, and excludes the poles of the integrand at $\alpha_{\circ}-1$ and $\alpha_{j}-1$ (for $\left.1 \leqslant j \leqslant t\right)$.

REMARK 6.23. Proposition 6.22 corresponds to the $q \rightarrow 1$ limit of analogous formulas in the $q$-Whittaker case stated as Corollary 4.1. However, since weak convergence does not imply convergence of moments in general, Proposition 6.22 cannot be deduced from Corollary 4.1.

Proof. Observe that

$$
\frac{\mathcal{G}(w)}{\mathcal{G}(w+1)}=e^{\tau w}\left(w+\alpha_{\circ}\right) \prod_{i=1}^{t}\left(w+\alpha_{i}\right) \prod_{j=1}^{n}\left(\frac{1}{w-\alpha_{j}}\right)
$$

and

$$
\frac{\overline{\mathcal{G}}(-w)}{\overline{\mathcal{G}}(-w-1)}=\frac{e^{\tau w}}{1+w-\alpha_{\circ}} \prod_{i=1}^{t}\left(\frac{1}{\alpha_{i}-w-1}\right) \prod_{j=1}^{n}\left(\frac{1}{w+\alpha_{j}}\right) .
$$

We use a similar approach to the proof of Proposition 3.5 using operators diagonalized by Whittaker functions. The eigenrelation (43) for Macdonald difference operator $\mathbf{D}_{n}^{1}$ becomes the following in the Whittaker limit (see [BC14, Lemma 4.1.36]).

$$
\sum_{i=1}^{n} \prod_{j \neq i} \frac{1}{\alpha_{i}-\alpha_{j}} \mathbf{T}_{i} \psi_{\mathbf{i} \alpha_{1}, \ldots, \mathbf{i} \alpha_{n}}(x)=e^{-x_{n}} \psi_{\mathbf{i} \alpha_{1}, \ldots, \mathbf{i} \alpha_{n}}(x),
$$

where $\mathbf{T}_{i}$ acts on functions in the variables $\alpha_{1}, \ldots, \alpha_{n}$ by shifting the $i$ th coordinate by 1 . Using $\psi_{w}\left(x_{1}, \ldots, x_{n}\right)=\psi_{-w}\left(-x_{n}, \ldots,-x_{1}\right)$, we obtain that

$$
\sum_{i=1}^{n} \prod_{j \neq i} \frac{1}{\alpha_{j}-\alpha_{i}} \mathbf{T}_{i}^{-1} \psi_{\mathbf{i} \alpha_{1}, \ldots, \mathbf{i} \alpha_{n}}(x)=e^{x_{1}} \psi_{\mathbf{i} \alpha_{1}, \ldots, \mathbf{i} \alpha_{n}}(x),
$$

where $\mathbf{T}_{i}^{-1}$ acts on functions in the variables $\alpha_{1}, \ldots, \alpha_{n}$ by shifting the $i$ th coordinate by -1 .

Let us iterate relation (138) $k$ times and use Proposition 6.10. We obtain

$$
\mathbb{E}\left[e^{-k T_{n}}\right]=\frac{1}{Z\left(\alpha_{1}, \ldots, \alpha_{n}\right)} \sum_{i_{1}, \ldots, i_{k}=1}^{n} \prod_{\ell=1}^{k}\left(\prod_{j \neq i_{\ell}} \frac{1}{\alpha_{i_{\ell}}-\alpha_{j}}\right) \mathbf{T}_{i_{\ell}} Z\left(\alpha_{1}, \ldots, \alpha_{n}\right),
$$


where

$$
Z\left(\alpha_{1}, \ldots, \alpha_{n}\right)=e^{\tau \sum_{i=1}^{n} \alpha_{i}^{2} / 2} \prod_{i=1}^{n} \Gamma\left(\alpha_{\circ}+\alpha_{i}\right) \prod_{j=n+1}^{t} \Gamma\left(\alpha_{j}+\alpha_{i}\right) \prod_{1 \leqslant i<j \leqslant n} \Gamma\left(\alpha_{i}+\alpha_{j}\right) .
$$

Similarly, if $\alpha_{i}>2 k$ and $\alpha_{i}+\alpha_{\circ}>k$ for all $1 \leqslant i \leqslant n$, we may iterate relation (139) $k$ times and use Proposition 6.10. We obtain,

$$
\mathbb{E}\left[e^{k T_{1}}\right]=\frac{1}{Z\left(\alpha_{1}, \ldots, \alpha_{n}\right)} \sum_{i_{1}, \ldots, i_{k}=1}^{n} \prod_{\ell=1}^{k}\left(\prod_{j \neq i_{\ell}} \frac{1}{\alpha_{j}-\alpha_{i_{\ell}}}\right) \mathbf{T}_{i_{\ell}}^{-1} Z\left(\alpha_{1}, \ldots, \alpha_{n}\right) .
$$

The assumptions on $\alpha_{i}$ 's ensure that one can apply Proposition 6.10 in the summand after applying (139) $k$ times. To conclude the proof of the proposition, we need to rewrite (140) and (141) as contour integrals, which can be done very similarly to Proposition 3.5.

6.4. Limits of $\boldsymbol{q}$-Whittaker dynamics. We may represent the ascending halfspace Whittaker process $T$ from Definition 6.9 as an array

$$
\begin{aligned}
& \begin{array}{lllll}
T_{n}^{(n)} & T_{n-1}^{(n)} & \cdots & T_{2}^{(n)} & T_{1}^{(n)}
\end{array} \\
& \begin{array}{lllll}
T_{n-1}^{(n-1)} & T_{n-2}^{(n-1)} & \cdots & T_{2}^{(n-1)} & T_{1}^{(n-1)}
\end{array} \\
& T_{2}^{(2)} \quad T_{1}^{(2)} \\
& T_{1}^{(1)}
\end{aligned}
$$

This triangular array is the (scaling) limit of the sequence of random $q$-Whittaker partitions $\lambda^{(t, 1)} \prec \cdots \prec \lambda^{(t, n)}$. From Sections 4.2 .2 and 4.2.3, there exist (multivariate) Markov dynamics, which map $\lambda^{(t, 1)} \prec \cdots \prec \lambda^{(t, n)}$ to $\lambda^{(t+1,1)} \prec$ $\cdots \prec \lambda^{(t+1, n)}$. In the $q \rightarrow 1$ limit, the triangular array is not interlacing anymore, but there should exist multivariate Markov dynamics transporting $T \in \mathbb{R}^{n(n+1) / 2}$ distributed as an ascending half-space Whittaker process to $T^{\prime} \in \mathbb{R}^{n(n+1) / 2}$ distributed as an ascending half-space Whittaker process with updated specializations, in such a way that the left edge and the right edge of the triangular array are both marginally Markov and correspond respectively to the limit of the dynamics from Sections 4.2.2 and 4.2.3. 


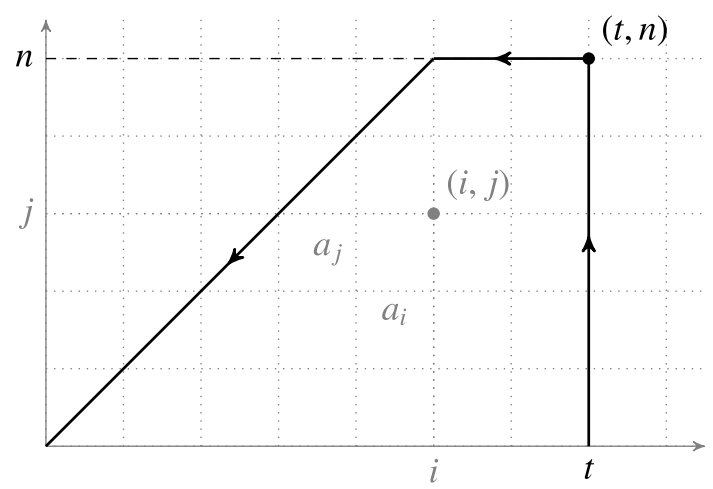

Figure 16. The path $\omega \in \Omega$ considered in Section 6.4.

In this section, we describe these limiting marginal dynamics. Connecting rigorously the resulting model to the law of $T_{1}^{(n)}$ and $T_{n}^{(n)}$ under the half-space Whittaker measure presents some technicalities (related to the fact that we must keep a positive Plancherel component when we work with half-space Whittaker measures) that we discuss in more detail in Section 6.6.

Throughout this section, we use the same scalings of parameters as in Section 6.2. We will often use the letter $\theta$ to denote the parameter of $q$-deformed probability distributions, and we will always scale this parameter as $\theta=e^{-\varepsilon \tilde{\theta}}$. Consider a half-space $q$-Whittaker process indexed by a path $\omega$ as in Figure 16 , where the sequence of specializations $\rho$ is chosen as in Section 4.2 (that is, edges $(i-1, j) \leftarrow(i, j)$ are labeled by single variable specialization $a_{i}$, edges $(i$, $j-1) \rightarrow(i, j)$ are labeled by single variable specialization $a_{j}$, and the diagonal edge is labeled by specialization $a_{\circ}$ ).

6.4.1. Right-edge dynamics. We are interested here in the scaling (as in [BC14, Theorem 4.2.4] and [MP17, Definition 8.4])

$$
\lambda_{j}^{(t, n)}=(t+n+1-2 j) \varepsilon^{-1} \log \varepsilon^{-1}+\varepsilon^{-1} \log \left(R_{j}^{\varepsilon}(t, n)\right),
$$

where $\lambda^{(t, n)}$ is distributed according to the half-space $q$-Whittaker measure $\mathbb{P}_{\left(a_{1}, \ldots, a_{n}\right),\left(a_{0}, a_{n+1}, \ldots, a_{t}\right)}^{q}$. We will show that under the scaling of parameters (111), the random variable $\left(R_{j}^{\varepsilon}(t, n)\right)_{1 \leqslant j \leqslant n}$ weakly converges to some random variable $\left(R_{j}(t, n)\right)_{1 \leqslant j \leqslant n}$.

Definition 6.24. The Gamma distribution with (shape) parameter $\tilde{\theta}$ (and scale parameter 1$)$, denoted by $\operatorname{Gamma}(\tilde{\theta})$, is the continuous probability distribution 
on $\mathbb{R}_{>0}$ with density

$$
\frac{1}{\Gamma(\tilde{\theta})} x^{\tilde{\theta}-1} e^{-x}
$$

Thus, if $X \sim \operatorname{Gamma}(\tilde{\theta})$ then $X^{-1}$ has the inverse-Gamma distribution, denoted by $\operatorname{Gamma}^{-1}(\tilde{\theta})$, and density

$$
\frac{1}{\Gamma(\tilde{\theta})} x^{-\tilde{\theta}-1} e^{-1 / x}
$$

Assume that we have sampled partitions $\lambda^{(v)}$ for $v$ belonging to or sitting below the path $\omega$ of Figure 16, according to the rules of Section 4.2 and using operators $\mathcal{U}^{\perp}$ and $\mathcal{U}_{\text {row }}^{\llcorner}$. For $1 \leqslant m \leqslant s \leqslant t$ and $m \leqslant n$, we have set $\lambda_{j}^{(s, m)}=(s+m+$ $1-2 j) \varepsilon^{-1} \log \varepsilon^{-1}+\varepsilon^{-1} \log \left(R_{j}^{\varepsilon}(s, m)\right)$. Theorem 8.7 in [MP17] shows that in the situation of the $q$-Whittaker process in the full-space case, the array $R^{\varepsilon}$ converges to some explicit limit $R$. In the course of proving this result, [MP17] shows that the dynamics $\mathcal{U}_{\text {row }}^{\llcorner}$have a limit in the following sense.

LEMMA 6.25 [MP17]. Assume that $\lambda_{j}^{(s-1, m)}, \lambda_{j}^{(s-1, m-1)}, \lambda_{j}^{(s, m-1)}$ are such that there exist real random variables $R_{j}(s-1, m), R_{j}(s-1, m-1)$ and $R_{j}(s$, $m-1)$ so that we have the weak convergences

$$
\begin{gathered}
R_{j}^{\varepsilon}(s-1, m-1) \underset{\varepsilon \rightarrow 0}{\Longrightarrow} R_{j}(s-1, m-1), \\
R_{j}^{\varepsilon}(s-1, m) \underset{\varepsilon \rightarrow 0}{\Longrightarrow} R_{j}(s-1, m), \\
R_{j}^{\varepsilon}(s, m-1) \underset{\varepsilon \rightarrow 0}{\Longrightarrow} R_{j}(s, m-1) .
\end{gathered}
$$

If $\lambda^{(s, m)}$ is sampled according to the dynamics $\mathcal{U}_{\text {row }}^{\llcorner}\left(\lambda^{(s, m)} \mid \lambda^{(s, m-1)}, \lambda^{(s-1, m-1)}\right.$, $\left.\lambda^{(s-1, m)}\right)$, then the sequence $R_{j}^{\varepsilon}(s, m)(1 \leqslant j \leqslant m)$ converges weakly as $\varepsilon$ goes to zero to some sequence $\left(R_{j}(s, m)\right)_{1 \leqslant j \leqslant m}$ whose distribution is explicit. In particular, $R_{1}(s, m)$ depends only on $R_{1}(s-1, m), R_{1}(s-1, m-1), R_{1}(s$, $m-1)$, and we have

$$
R_{1}(s, m)=d_{s m}\left(R_{1}(s, m-1)+R_{1}(s-1, m)\right)
$$

, where $d_{s m}$ is a $\mathrm{Gamma}^{-1}\left(\alpha_{m}+\alpha_{s}\right)$ random variable, the random variables $\left(d_{s m}\right)_{s, m}$ are independent, and we adopt the convention that $R_{1}(0, j)=R_{1}(t$, $0)=0$.

In order to have a recurrence characterizing the law of $R(t, j)$ completely, we examine the degeneration of the boundary operator $\mathcal{U}^{L}$ as $\varepsilon \rightarrow 0$. We will only 
focus on the projection to the first coordinate $\lambda_{1}$ (that is, $R_{1}$ in the limit), which is marginally Markov (by Lemma 4.15).

Lemma 6.26 [MP17, Lemma 8.15]. Let $X_{\varepsilon}$ be a $\mathbb{Z}_{\geqslant 0}$-family of random variables with $\mathrm{qGeom}(\theta)$ distribution and set

$$
X_{\varepsilon}=\varepsilon^{-1} \log \left(\Gamma_{\varepsilon}\right)+\varepsilon^{-1} \log \left(\varepsilon^{-1}\right) .
$$

Then, if $\theta=e^{-\varepsilon \tilde{\theta}}, \Gamma_{\varepsilon}$ weakly converges to a $\mathrm{Gamma}^{-1}(\tilde{\theta})$ random variable.

Lemma 6.26 applied to the result of Lemma 4.15 implies that in the $\varepsilon \rightarrow 0$ limit, for $t \geqslant 2$

$$
R_{1}(t, t)=d_{t t} R_{1}(t, t-1),
$$

where $d_{t t}$ is a Gamma ${ }^{-1}\left(\alpha_{\circ}+\alpha_{t}\right)$ random variable, and $R(1,1)=d_{11}$.

Proposition 6.27. For any path $\omega \in \Omega$, the sequence $\left(R_{1}^{\varepsilon}(t, n)\right)_{(t, n) \in \omega}$ defined by (142) converges in distribution as $\varepsilon$ goes to zero to a sequence $(R(t, n))_{(t, n) \in \omega}$ such that

$$
(Z(t, n))_{(t, n) \in \omega} \stackrel{(d)}{=}(R(t, n))_{(t, n) \in \omega},
$$

where $Z(t, n)$ is the partition function of the half-space log-gamma directed polymer (Definition 1.1). In particular, under the notations above,

$$
(1-q)^{t+n-1} q^{-\lambda_{1}^{(t, n)}} \underset{q \rightarrow 1}{\Longrightarrow} Z(t, n) .
$$

Proof. The convergence in distribution is proved by Lemmas 6.25 and 6.26. Moreover, $Z(n, m)$ and $R(n, m)$ satisfy the same recurrence relation in law, and hence have the same distribution.

6.4.2. Left-edge dynamics. We consider now the scaling

$$
\lambda_{t}^{(t, j)}=(t-j+1) \varepsilon^{-1} \log \varepsilon^{-1}-\varepsilon^{-1} \log \left(L^{\varepsilon}(t, j)\right),
$$

where $\lambda^{(t, j)}$ is distributed according to the half-space $q$-Whittaker measure $\mathbb{P}_{\left(a_{1}, \ldots, a_{j}\right),\left(a_{0}, a_{j+1}, \ldots, a_{t}\right)}^{q}$. We can show in a way similar to the right-edge case that $L^{\varepsilon}(t, j)$ weakly converges to some $L(t, j)$, where the family of random variables $\{L(t, j)\}_{1 \leqslant j \leqslant t}$ has an explicit recursive description. In a way similar to the rightedge dynamics, the proof of Theorem 8.8 in [MP17] implies that when $t>j$,

$$
L(t, j)=L(t-1, j-1)+g_{t, j} L(t-1, j),
$$

where $g_{t, j}$ is a $\operatorname{Gamma}\left(\alpha_{t}+\alpha_{j}\right)$ random variable and the family $\left(g_{t, j}\right)$ consists of independent members.

The limit of the boundary dynamics $\mathcal{U}^{L}$ is more complicated. 
LEMMA 6.28. Let $X_{\varepsilon}$ be a q-inverse Gaussian random variable with parameters $m$ and $\theta$ (see Definition 4.16). Assume that $\theta=e^{-\varepsilon \tilde{\theta}}$ and $m=2 \varepsilon^{-1} \log \left(\varepsilon^{-1}\right)-$ $\varepsilon^{-1} \log (L)$. Then, letting $X_{\varepsilon}=\varepsilon^{-1} \log \left(\varepsilon^{-1}\right)-\varepsilon^{-1} \log \left(Y_{\varepsilon}\right)$, the random variable $Y_{\varepsilon}$ weakly converges to the continuous random variable on $\mathbb{R}_{>0}$ with density

$$
\frac{1}{2 L^{\tilde{\theta} / 2} K_{\theta}(2 \sqrt{L})} x^{\tilde{\theta}-1} e^{-x-L / x},
$$

where $K_{\theta}$ is the modified Bessel function of the second kind, which is a particular case of the generalized inverse Gaussian distribution. (Sometimes $K_{\theta}$ are called Macdonald functions, although they are completely different from Macdonald symmetric functions.)

Proof. By definition, for $y$ such that $\varepsilon^{-1} \log \left(\varepsilon^{-1}\right)-\varepsilon^{-1} y \in\{0,1, \ldots, m\}$,

$$
\begin{aligned}
& \mathbb{P}\left(\log \left(Y_{\varepsilon}\right)=y\right)=\mathbb{P}\left(X_{\varepsilon}=\varepsilon^{-1} \log \left(\varepsilon^{-1}\right)-\varepsilon^{-1} y\right) \\
& \quad=\frac{1}{Z_{m}(\theta)} \theta^{\varepsilon^{-1} \log \left(\varepsilon^{-1}\right)-\varepsilon^{-1} y} \frac{(q ; q)_{2 \varepsilon^{-1} \log \left(\varepsilon^{-1}\right)-\varepsilon^{-1} \log (L)}}{(q ; q)_{\varepsilon^{-1} \log \left(\varepsilon^{-1}\right)-\varepsilon^{-1} y}(q ; q)_{\varepsilon^{-1} \log \left(\varepsilon^{-1}\right)-\varepsilon^{-1}(\log (L)-y)}} \\
& \quad=\frac{\varepsilon^{\tilde{\theta}}}{Z_{m}(\theta)} \exp \left(y \tilde{\theta}-e^{y}-L / e^{y}-\mathcal{A}(\varepsilon)+o(1)\right),
\end{aligned}
$$

where we have used estimates (113) and (114) from Lemma 6.4 in the last equality (the error goes to zero uniformly for $y$ in a compact set). In order to conclude that $\log \left(Y_{\varepsilon}\right)$ converges to the random variable with density proportional to $\exp \left(y \tilde{\theta}-e^{y}-L / e^{y}\right)$, we need to prove some tail decay estimate when $|y| \rightarrow \infty$, uniformly in $\epsilon$, showing that the sequence $\log \left(Y_{\varepsilon}\right)$ is tight. Using inequality (115) in Lemma 6.4, we have that for any $y \in \mathbb{R}$ and any fixed $L>0$,

$$
\mathbb{P}\left(\log \left(Y_{\varepsilon}\right)=y\right) \leqslant \frac{\varepsilon^{\tilde{\theta}}}{Z_{m}(\theta)} \exp \left(y \tilde{\theta}-e^{y}-L / e^{y}-\mathcal{A}(\varepsilon)+o(1)\right),
$$

where the error $o$ (1) now goes to zero as $\varepsilon \rightarrow 0$ uniformly for any $y \in \mathbb{R}$. Thus, $\log \left(Y_{\varepsilon}\right)$ weakly converges to the distribution on $\mathbb{R}$ with density

$$
\frac{1}{C} e^{y \tilde{\theta}} e^{-e^{y}-L / e^{y}},
$$

where $C$ is a normalizing constant so that the density integrates to 1 . By the change of variables $x=e^{y}$, it implies that $Y_{\varepsilon}$ converges to the continuous random variable on $\mathbb{R}$ with density

$$
\frac{1}{C} x^{\tilde{\theta}-1} e^{-x-L / x} .
$$


This distribution is known as a particular case of the generalized inverse Gaussian distribution, and it is well known that the normalizing constant $C$ equals $2 L^{\tilde{\theta} / 2} K_{\theta}(2 \sqrt{L})$ [Jor82].

Lemma 6.28 applied to the result of Lemma 4.17 implies that in the $\varepsilon \rightarrow 0$ limit, for $t \geqslant 2, L(t, t)$ has the generalized inverse Gaussian distribution with parameters $L(t, t-1)$ and $(-1)^{t+1} \alpha_{\circ}+\alpha_{t}$ as defined in Lemma 6.28.

REMARK 6.29. One could define, as in Section 6.4.1, a sort of directed polymer model such that its partition function satisfies the recurrence

$$
\begin{cases}L(t, j)=L(t-1, j-1)+g_{t, j} L(t-1, j) & \text { for } t>j, \\ L(t, t)=h_{t}\left(L(t, t-1),(-1)^{t-1} \alpha_{\circ}+\alpha_{t}\right) & \text { for } t \geqslant 2,\end{cases}
$$

where $\left\{g_{i, j}\right\}_{i>j}$ is a family of independent $\operatorname{Gamma}\left(\alpha_{i}+\alpha_{j}\right)$ distributed random variables, and $h_{t}(L, \alpha)$ denotes an inverse Gaussian random variable (independent for each $t$ ) with parameters $L$ and $\alpha$ as defined in Lemma 6.28.

We may also deduce the following asymptotics for Rogers-Szegó polynomials defined in (94).

COROllary 6.30. Consider the mth Rogers-Szegó polynomial $Z_{m}(\theta)$ under the scalings $\theta=q^{\tilde{\theta}}$ and $q^{m}=L(1-q)^{2}$ for some fixed $L>0$. Then, we have that

$$
Z_{m}(\theta) \frac{(q ; q)_{\infty}}{(1-q)^{\tilde{\theta}-1}} \underset{q \rightarrow 1}{\longrightarrow} 2 L^{\tilde{\theta} / 2} K_{\tilde{\theta}}(2 \sqrt{L})
$$

where $K_{\theta}$ is the modified Bessel function of the second kind.

Proof. The result is obtained by matching the normalizing constants in the proof of Lemma 6.28.

6.5. Whittaker measure and geometric RSK. Let us examine in more detail the limit of the half-space $q$-Whittaker measure as $q \rightarrow 1$, when $\mathcal{E}_{\lambda}$ is specialized into a single variable and the Plancherel component is set to $\gamma=0$. In that case, we can define the $\tau=0$ analogue of $\mathcal{T}_{\alpha_{o}}{ }^{\tau}$, which we will naturally denote by $\mathcal{T}_{\alpha_{\circ}}^{0}$.

Assuming for simplicity that $n$ is even,

$$
\mathcal{E}_{\lambda_{1}, \ldots, \lambda_{n}}\left(a_{\circ}\right)=a_{\circ}^{\sum \lambda_{2 i-1}-\lambda_{2 i}} \prod_{i=1}^{n} \frac{1}{(q ; q)_{\lambda_{i}-\lambda_{i+1}}}
$$




$$
=\varepsilon^{n \alpha} e^{-\alpha_{\circ} \sum_{i=1}^{n / 2} T_{2 i-1}-T_{2 i}} \prod_{i=1}^{n} \frac{1}{(q ; q)_{\lambda_{i}-\lambda_{i+1}}} .
$$

Thus, we have

$$
\mathcal{E}_{\lambda}\left(a_{\circ}\right)=\varepsilon^{n \alpha_{\circ}} e^{-\alpha_{\circ} \sum_{i=1}^{n / 2} T_{2 i-1}-T_{2 i}} e^{-n \mathcal{A}(\varepsilon)} e^{-e^{-T_{n}}} .
$$

Taking into account a Jacobian of $\varepsilon^{-n}$, we arrive at

$$
\mathcal{T}_{\alpha_{\circ}}^{0}(T)=e^{-\alpha_{\diamond} \sum_{i=1}^{n / 2} T_{2 i-1}-T_{2 i}} e^{-e^{-T_{n}}},
$$

and the half-space Whittaker measure can be extended to $\tau=0$ with

$$
\mathbb{P}(d T)=d T \frac{\psi_{\mathbf{i} \alpha_{1}, \ldots, \mathbf{i} \alpha_{n}}(T) e^{-\alpha_{\circ} \sum_{i=1}^{n / 2} T_{2 i-1}-T_{2 i}} e^{-e^{-T_{n}}}}{\prod_{i=1}^{n} \Gamma\left(\alpha_{i}+\alpha_{\circ}\right) \prod_{1 \leqslant i<j \leqslant n} \Gamma\left(\alpha_{i}+\alpha_{j}\right)} .
$$

REMARK 6.31. Using the identity

$$
\mathcal{E}_{\lambda}(a)=\frac{1}{\Phi(a)} \sum_{\mu} \mathcal{E}_{\mu}(0) P_{\mu / \lambda}(a),
$$

it should be possible to make sense of $\mathcal{T}_{\alpha}^{\tau}$ for $\tau=0$ and arbitrary $\alpha_{1}, \ldots, \alpha_{k}>0$.

The following result from [OSZ14] shows that $\mathbb{P}$ is a well-defined probability measure.

THEOREM 6.32 [OSZ14, Corollary 5.4]. For $\mathfrak{R e}\left(\alpha_{\circ}+\alpha_{i}\right)>0$ and $\mathfrak{R e}\left(\alpha_{i}+\alpha_{j}\right)>$ 0 for all $i, j$, and $n$ even

$$
\int_{\mathbb{R}^{n}} \psi_{\mathbf{i} \alpha_{1}, \ldots, \mathbf{i} \alpha_{n}}(T) e^{-\alpha_{\circ} \sum_{i=1}^{n / 2} T_{2 i-1}-T_{2 i}} e^{-e^{-T_{n}}} d T=\prod_{i=1}^{n} \Gamma\left(\alpha_{i}+\alpha_{\circ}\right) \prod_{1 \leqslant i<j \leqslant n} \Gamma\left(\alpha_{i}+\alpha_{j}\right) .
$$

Using [OSZ14] (in particular Equation (2.2) and Corollary 5.3 therein), the measure $\mathbb{P}$ corresponds (modulo a shift of variables by $\log (2)$ ) to the pushforward via the geometric RSK algorithm of a symmetric matrix of weights $\left\{\tilde{w}_{i, j}\right\}_{1 \leqslant i, j \leqslant n}$ having the following distribution: For $1 \leqslant i<j \leqslant n, \tilde{w}_{i, j}$ is distributed as an inverse-Gamma random variable with shape parameter $\alpha_{i}+\alpha_{j}$ and scale parameter $1, \tilde{w}_{i, i}$ is distributed as an inverse-Gamma random variable with shape parameter $\alpha_{i}+\alpha_{\circ}$ and scale parameter $1 / 2$, and $\tilde{w}_{i, j}$ are independent modulo the symmetry constraint that for all $i, j$ we have $\tilde{w}_{i, j}=\tilde{w}_{j, i}$. (We use the convention that if $X$ is an inverse-Gamma random variable with scale parameter 
$1, k X$ is an inverse-Gamma random variable with scale parameter $k$ and the same shape parameter.)

Using properties of the geometric RSK algorithm, [OSZ14] shows that $t_{n, n}$ (one of the components of the image of $\tilde{w}$ by the geometric RSK algorithm) has the law of the partition function $\tilde{Z}(n, n)$ of a polymer model with symmetrized weights $\left\{w_{i, j}\right\}_{1 \leqslant i, j \leqslant n}$ as above. The change of scale parameter on the diagonal compensates the fact that there are more paths in the symmetric model than in the half-space models. Counting the paths carefully, we arrive at $2 \tilde{Z}(n, n)=Z(n$, $n)$, where $Z(n, n)$ is the partition function of the log-gamma polymer in a halfquadrant (Definition 1.1). This also shows that

$$
T_{1} \stackrel{(d)}{=} \log Z(n, n),
$$

The relation between our half-space Whittaker measure and the Whittaker measure from [OSZ14] holds not only for the marginal $T_{1}$. Indeed, under the Whittaker measure,

$$
\mathbb{E}[f(T)]=\frac{\int_{\mathbb{R}^{n}} f\left(e^{T_{1}}, \ldots, e^{T_{n}}\right) \psi_{\mathbf{i} \alpha}(T) e^{-\alpha_{\circ} \sum_{i=1}^{n / 2} T_{2 i-1}-T_{2 i}} e^{-e^{-T_{n}}} d T}{\prod_{i=1}^{n} \Gamma\left(\alpha_{i}+\alpha_{\circ}\right) \prod_{1 \leqslant i<j \leqslant n} \Gamma\left(\alpha_{i}+\alpha_{j}\right)} .
$$

Making the change of variables $T_{i}=\tilde{T}_{i}+\log (2)$ for all $i$, we obtain that

$$
\begin{aligned}
\mathbb{E}[f(T)] & =\frac{\int_{\mathbb{R}^{n}} f\left(2 e^{T_{1}}, \ldots, 2 e^{T_{n}}\right) \psi_{\mathbf{i} \alpha}(T) e^{-\alpha_{\circ} \sum_{i=1}^{n / 2} T_{2 i-1}-T_{2 i}} e^{-\frac{1}{2} e^{-T_{n}}} d T}{2^{\sum_{i} \alpha_{i}} \prod_{i=1}^{n} \Gamma\left(\alpha_{i}+\alpha_{\circ}\right) \prod_{1 \leqslant i<j \leqslant n} \Gamma\left(\alpha_{i}+\alpha_{j}\right)} \\
& =\int f\left(2 t_{1 n}, \ldots, 2 t_{n n}\right) \tilde{v}_{\alpha, \zeta}(d w),
\end{aligned}
$$

where the measure $\tilde{v}_{\alpha, \zeta}(d w)$ is defined in [OSZ14, (5.10)].

We will see in the next section another way to relate the Whittaker measure with the distribution of directed polymers.

6.6. Half-space directed polymers and Whittaker measure. In Section 6.4, we have studied the limit of $q$-Whittaker dynamics in the scaling in which the $q$-Whittaker measure becomes the Whittaker measure. In particular, we found that the limiting dynamics of $q^{-\lambda_{1}}$ are the same as that of the partition function of the log-gamma polymer. Hence, it is natural to expect that the marginal $T_{1}$, for $T$ distributed according to some Whittaker measure has the same distribution as $\log (Z(t, n))$, where $Z(t, n)$ is the log-gamma partition function (Definition 1.1). However, we have proved the convergence of the $q$-Whittaker measure to the Whittaker measure only when the Plancherel component of the specialization is 
positive (Proposition 6.18). Further, we have not defined the Whittaker measure corresponding to $Z(t, n)$ when $\tau=0$ (outside of the special case of Section 6.5) and it is not clear how to take a limit of (118) as $\tau \rightarrow 0$.

There are two cases where it is possible to relate rigorously the Whittaker measure with the limit of $q$-Whittaker Markov dynamics as $q$ goes to 1 .

6.6.1. Partition function on the boundary. When $n=t$, we consider the halfspace $q$-Whittaker measure $\mathbb{P}_{\left(a_{1}, \ldots, a_{n}\right),\left(a_{\circ}\right)}^{q}$ and the Whittaker measure $\mathbb{P}_{\left(\alpha_{1}, \ldots, \alpha_{n}\right),\left(\alpha_{\circ}\right)}$, which takes a simple form as in Section 6.5. In that particular case, we know from Theorem 6.32 that the Whittaker measure is a well-defined probability measure, and since the density of the $q$-Whittaker measure converges to the density of the Whittaker measure, we have the convergence

$$
\mathbb{P}_{\left(a_{1}, \ldots, a_{n}\right),\left(a_{\circ}\right)}^{q} \underset{q \rightarrow 1}{\Longrightarrow} \mathbb{P}_{\left(\alpha_{1}, \ldots, \alpha_{n}\right),\left(\alpha_{\circ}\right)} .
$$

Hence, using Proposition 6.27, we recover that for $T$ distributed according to $\mathbb{P}_{\left(\alpha_{1}, \ldots, \alpha_{n}\right),\left(\alpha_{0}\right)}$, we have that $T_{1} \stackrel{(d)}{=} \log Z(n, n)$, where $Z(n, n)$ is the partition function of the log-gamma polymer at point $(n, n)$.

6.6.2. Limits of $q$-Whittaker dynamics with additional Plancherel specialization. Another approach is to extend the results of Section 6.4 to $q$-Whittaker processes including a Plancherel component. More precisely, we consider a half-space $q$-Whittaker process indexed by a path $\omega$ as in Figure 15 . For all $i \leqslant t$ and any $j$, edges $(i-1, j) \leftarrow(i, j)$ are labeled by single variable specialization $a_{i}$, for any $i, j$, edges $(i, j-1) \rightarrow(i, j)$ are labeled by single variable specialization $a_{j}$, the diagonal edge is labeled by specialization $a_{\circ}$ and now, edges $(t, j) \leftarrow(t+1, j)$ are labeled by the Plancherel specialization $\tau$. We consider only the right-edge dynamics (the last parts of partitions).

DEFINITION 6.33. Let $\left(w_{i, j}\right)_{1 \leqslant i \leqslant j}$ be a family of inverse-Gamma random variables as in Definition 1.1. Let $\left(B^{i}(s)\right)_{i \geqslant 1, s>0}$ be a family of independent standard Brownian motions with drift $b_{i}$.

Let $t \geqslant n \geqslant 1$. We define the hybrid half-space log-gamma/semidiscrete directed polymer as a measure on the concatenation of two paths $\pi, \phi$ (see Figure 17). The path $\pi$ progresses from $(1,1)$ by unit up-right steps in the halfquadrant until a certain point $\left(t, n_{0}\right)$. The path $\phi$, depending on a parameter $\tau>0$, is a semidiscrete path encoded by a set $t=\tau_{0}<\tau_{1}<\cdots<\tau_{n-n_{0}}<$ $\tau_{n-n_{0}+1}=t+\tau$. The path progresses horizontally from $\left(t, n_{0}\right)$ to $\left(\tau_{1}, n_{0}\right)$, jumps to $\left(\tau_{1}, n_{0}+1\right)$ and then progresses to $\left(\tau_{2}, n_{0}+1\right)$ and so on until it reaches $(\tau+t, n)$. 


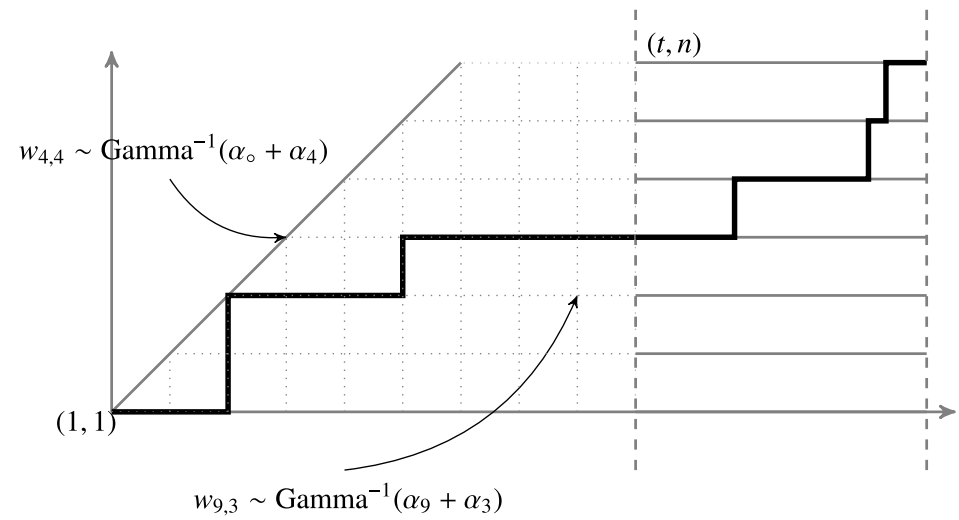

Figure 17. An admissible path in the hybrid half-space log-gamma/semidiscrete directed polymer.

The probability density of such a hybrid path $\pi, \phi$ is given by

$$
\frac{1}{Z(t, n, \tau)} \prod_{(i, j) \in \pi} w_{i, j} \exp \left(\int_{0}^{\tau} d B^{\phi(t+s)}(s)\right),
$$

where the partition function $Z(t, n, \tau)$ is defined by

$$
\begin{aligned}
Z(t, n, \tau)= & \sum_{n_{0}=1}^{n} \sum_{\pi:(1,1) \rightarrow\left(t, n_{0}\right)} \prod_{(i, j) \in \pi} w_{i, j} \\
& \times \int_{t<\tau_{1}<\cdots<\tau_{n-n_{0}-1}<t+\tau} \exp \left(\int_{0}^{\tau} d B^{\phi(t+s)}(s)\right) d \tau_{1} \ldots d \tau_{n-n_{0}-1} .
\end{aligned}
$$

Note that $Z(t, n, \tau)$ can be constructed in a recursive way by constructing first $Z(t, m, 0)$ for all $1 \leqslant m \leqslant n$ as in Section 6.4.1. Then [O'C12] showed that the vector of free energies $(\log (Z(t, m, \tau)))_{1 \leqslant m \leqslant n}$ is the solution to the following system of stochastic differential equations (SDEs) with time parameter $\tau$ and initial data $(\log (Z(t, m, 0)))_{1 \leqslant m \leqslant n}$ :

$$
\left\{\begin{array}{l}
d T_{1}^{(1)}=d B_{\tau}^{1} \\
d T_{1}^{(m)}=d B_{\tau}^{m}+e^{T_{1}^{(m-1)}-T_{1}^{(m)}} d \tau \quad \text { for all } 2 \leqslant m \leqslant n .
\end{array}\right.
$$

The degeneration of the RSK dynamics $\mathcal{U}_{\text {row }}^{\llcorner}$from Section 2.4 where the horizontal specialization becomes a Plancherel specialization was studied in 
[BP16]. Some of the arguments presented in [BP16, Section 8.4] are only heuristics, and the convergence of $q$-Whittaker dynamics to SDE is not proved for the whole triangular array. However, the convergence is shown in [BP16, Section 8.4.4] for the first coordinate marginal $T_{1}^{(k)}$. More precisely, for any fixed $n$, under the Whittaker dynamics, which correspond to the $q \rightarrow 1$ limit of $\mathcal{U}_{\text {row }}^{\llcorner}$, $\left(T_{1}^{(k)}\right)_{1 \leqslant k \leqslant n}$ satisfies SDE (144) with the Brownian motion drifts $b_{i}$ chosen as $-\alpha_{i}$. Thus, we arrive at the following.

PROpOSITION 6.34. Let $T$ be distributed according to $\mathbb{P}_{\left(\alpha_{1}, \ldots, \alpha_{n}\right),\left(\alpha_{\circ}, \alpha_{n+1}, \ldots, \alpha_{t}, \tau\right)}$ with $\tau>0$. Let $Z(t, n, \tau)$ be the partition function of the hybrid half-space log-gamma/semidiscrete directed polymer as in Definition 6.33, where the drifts in the hybrid log-gamma/semidiscrete model are chosen as $b_{i}=-\alpha_{i}$. Then, we have the distributional equality

$$
T_{1}^{(n)} \stackrel{(d)}{=} \log Z(t, n, \tau) .
$$

REMARK 6.35. We expect that (145) should hold as well when $\tau=0$ in some sense (see Remark 6.31 about the case $\tau=0$ ). While it is clear from the definition of the polymer partition function that $Z(t, n, \tau)$ converges to $Z(t$, $n, 0)$, additional arguments are necessary to control the tails of the half-space Whittaker measure as $\tau \rightarrow 0$.

COROLlaRY 6.36. Let $t \geqslant n \geqslant 1, \alpha_{1}, \ldots, \alpha_{t}>0$ and $\alpha_{\circ}>0$. For all $k \in \mathbb{Z}_{>0}$ such that $k<\min \left\{2 \alpha_{i}, \alpha_{\circ}+\alpha_{i}\right\}$,

$$
\begin{aligned}
\mathbb{E}\left[Z(t, n)^{k}\right]= & \oint \frac{d w_{1}}{2 \mathbf{i} \pi} \cdots \oint \frac{d w_{k}}{2 \mathbf{i} \pi} \prod_{1 \leqslant a<b \leqslant k} \frac{w_{a}-w_{b}}{w_{a}-w_{b}-1} \frac{1+w_{a}+w_{b}}{2+w_{a}+w_{b}} \\
& \times \prod_{m=1}^{k} \frac{1+2 w_{m}}{1+w_{m}-\alpha_{\circ}} \prod_{i=1}^{t}\left(\frac{1}{\alpha_{i}-w_{m}-1}\right) \prod_{j=1}^{n}\left(\frac{1}{w_{m}+\alpha_{j}}\right),
\end{aligned}
$$

where the positively oriented contours are such that for all $1 \leqslant c \leqslant k$, the contour for $w_{c}$ encloses $\left\{-\alpha_{j}\right\}_{1 \leqslant j \leqslant n}$ and $\left\{w_{c+1}+1, \ldots, w_{k}+1\right\}$, and excludes the poles of the integrand at $\alpha_{\circ}-1$ and $\alpha_{j}-1$ (for $1 \leqslant j \leqslant t$ ).

Proof. Combining the moment formula for $e^{T_{1}}$ from Proposition 6.22 with Proposition 6.34 yields a formula for $\mathbb{E}\left[Z(t, n, \tau)^{k}\right]$. Then, we may take the limit when $\tau$ goes to zero in the formulas, but we need to prove that the moments converge as well as $\tau \rightarrow 0$. The hybrid partition function $Z(t, n, \tau)$ converges almost surely as $\tau$ goes to zero to the partition function of the half-space loggamma polymer $Z(t, n)=Z(t, n, 0)$ (if $Z(t, n, \tau)$ and $Z(t, n)$ are defined with 
the same variables $w_{i, j}$ ). Let $\tau^{*}>0$ be an arbitrary positive real number. Using the system of SDEs (144), we obtain that for $0 \leqslant \tau<\tau^{*}$,

$$
\log \left(Z\left(t, n, \tau^{*}\right)\right)-\log (Z(t, n, \tau))=B_{\tau^{*}}^{(n)}-B_{\tau}^{(n)}+\int_{\tau}^{\tau^{*}} \frac{Z(t, n-1, s)}{Z(t, n, s)} d s .
$$

Hence, since $\frac{Z(t, n-1, s)}{Z(t, n, s)} \geqslant 0$, we have that for $\tau^{*}>0$ fixed and any $0 \leqslant \tau<\tau^{*}$,

$$
0 \leqslant Z(t, n, \tau) \leqslant Z\left(t, n, \tau^{*}\right) \exp \left(\sup _{0 \leqslant s \leqslant \tau^{*}}\left\{B_{s}^{(n)}\right\}-B_{\tau^{*}}^{(n)}\right) .
$$

For $k<\min \left\{2 \alpha_{i}, \alpha_{\circ}+\alpha_{i}\right\}$, we know from Proposition 6.22 that $\mathbb{E}\left[Z\left(t, n, \tau^{*}\right)\right]^{k}<$ $\infty$. The random variable $\exp \left(\sup _{0 \leqslant s \leqslant \tau^{*}}\left\{B_{s}^{(n)}\right\}-B_{\tau^{*}}^{(n)}\right)$ has finite moments of all orders, since $\sup _{0 \leqslant s \leqslant \tau^{*}}\left\{B_{s}^{(n)}\right\}-B_{\tau^{*}}^{(n)}$ is distributed as the absolute value of a Gaussian ([RY13, Ch. III, Ex. 3.14]). Thus, the right-hand side of (148) does not depend on $\tau$ and has finite moment of order $k$ as long as $k<\min \left\{2 \alpha_{i}, \alpha_{\circ}+\alpha_{i}\right\}$. Dominated convergence implies that

$$
\mathbb{E}\left[Z(t, n, \tau)^{k}\right] \underset{\tau \rightarrow 0}{\longrightarrow} \mathbb{E}\left[Z(t, n, 0)^{k}\right]
$$

which concludes the proof.

REMARK 6.37. The moment formula (146) is reminiscent of nested contour integral solutions of the delta Bose gas in a half-space; see for instance [BBC16, Lemma 4.2] and Proposition 7.1 in the present paper. We believe that the formula above could as well be obtained by solving the system of difference equations satisfied by the function

$$
\left(n_{1}, \ldots, n_{k}\right) \mapsto \mathbb{E}\left[Z\left(t, n_{1}\right) \ldots Z\left(t, n_{k}\right)\right]
$$

6.7. Alternative derivation of Laplace transform integral formulas. We can also use our Whittaker eigenrelations for $\mathbf{B}$ and $\overline{\mathbf{B}}$ (defined in (109) and (110)) to directly compute Laplace transforms. The principle is to act with $\mathbf{B}$ and $\overline{\mathbf{B}}$ on both sides of the Whittaker analogue of the generalized Littlewood identity from Proposition 6.10, We will need to justify that one can exchange the action of the operators with the integrations so that we may use our eigenrelations (107) and (108). For the latter, it is essential to keep a positive Plancherel specialization (we could not adapt the same approach using the identity from Theorem 6.32, which would correspond to the $\tau=0$ case). 
THeOREM 6.38. Fix $n \leqslant t, \tau>0$ and $\alpha_{1}, \ldots, \alpha_{t}>0$ and $\alpha_{\circ} \in \mathbb{R}$ such that $\alpha_{\circ}+\alpha_{i}>0$ for $1 \leqslant i \leqslant n$. Under the Whittaker measure $\mathbb{P}_{\left(\alpha_{1}, \ldots, \alpha_{n}\right),\left(\alpha_{\circ}, \alpha_{n+1}, \ldots, \alpha_{t}, \tau\right)}$, we have

$$
\begin{aligned}
\mathbb{E}\left[e^{-u e^{T_{1}}}\right]= & \int_{\left(\mathcal{D}_{-r}\right)^{n}} \frac{d z}{(2 \mathbf{i} \pi)^{n}} \mathfrak{m}_{n}(\mathbf{i} z)(2 \pi)^{n} \prod_{i, j=1}^{n} \Gamma\left(-z_{i}-\alpha_{j}\right) \prod_{1 \leqslant i<j \leqslant n} \frac{\Gamma\left(-z_{i}-z_{j}\right)}{\Gamma\left(\alpha_{i}+\alpha_{j}\right)} \\
& \times \prod_{i=1}^{n}\left(u^{\alpha_{i}+z_{i}} e^{\tau\left(z_{i}^{2}-\alpha_{i}^{2}\right) / 2} \frac{\Gamma\left(\alpha_{\circ}-z_{i}\right)}{\Gamma\left(\alpha_{\circ}+\alpha_{i}\right)} \prod_{j=n+1}^{t} \frac{\Gamma\left(\alpha_{j}-z_{i}\right)}{\Gamma\left(\alpha_{j}+\alpha_{i}\right)}\right),
\end{aligned}
$$

where $\mathfrak{m}_{n}$ is defined in (104), and $r>0$ is such that $r+\alpha_{\circ}>0$ and $r>\alpha_{i}$ for all $1 \leqslant i \leqslant n$.

We also have

$$
\begin{aligned}
\mathbb{E}\left[e^{-u e^{-T_{n}}}\right]= & \int_{\left(\mathcal{D}_{r}\right)^{n}} \frac{d z}{(2 \mathbf{i} \pi)^{n}} \mathfrak{m}_{n}(\mathbf{i} z)(2 \pi)^{n} \prod_{i, j=1}^{n} \Gamma\left(-z_{i}+\alpha_{j}\right) \prod_{1 \leqslant i<j \leqslant n} \frac{\Gamma\left(z_{i}+z_{j}\right)}{\Gamma\left(\alpha_{i}+\alpha_{j}\right)} \\
& \times \prod_{i=1}^{n}\left(u^{-a_{i}-z_{i}} e^{\tau\left(z_{i}^{2}-\alpha_{i}^{2}\right) / 2} \frac{\Gamma\left(z_{i}+\alpha_{\circ}\right)}{\Gamma\left(\alpha_{i}+\alpha_{\circ}\right)} \prod_{j=n+1}^{t} \frac{\Gamma\left(z_{i}+\alpha_{j}\right)}{\Gamma\left(\alpha_{i}+\alpha_{j}\right)}\right), \quad(150)
\end{aligned}
$$

where $r>0$ is such that $r+\alpha_{\circ}>0$ and $r<\alpha_{i}$ for all $1 \leqslant i \leqslant n$.

Proof. Recall the statement of Proposition 6.10: for $w \in \mathbb{H}^{n}$,

$$
\begin{aligned}
& \int_{\mathbb{R}^{n}} d x \psi_{w}(x) \mathcal{T}_{\alpha_{\circ}, \alpha_{n+1}, \ldots, \alpha_{t}}^{\tau}(x) \\
& \quad=e^{-\tau \sum_{i=1}^{n} w_{i}^{2} / 2} \prod_{i=1}^{n} \Gamma\left(\alpha_{\circ}-\mathbf{i} w_{i}\right) \prod_{j=n+1}^{t} \Gamma\left(\alpha_{j}-\mathbf{i} w_{i}\right) \prod_{1 \leqslant i<j \leqslant n} \Gamma\left(-\mathbf{i}\left(w_{i}+w_{j}\right)\right) .
\end{aligned}
$$

We may substitute $w_{i}+\mathbf{i} r$ in place of $w_{i}$ above, for $r>0$. The identity remains true as long as $w_{i}+\mathbf{i} r \in \mathbb{H}$ for all $i$ and $\mathfrak{R e}\left[\alpha_{\circ}-\mathbf{i} w_{i}+r\right]>0$, but $w$ does not necessarily belong to $\mathbb{H}^{n}$ anymore. Using the shift property $\psi_{w+\mathbf{i} r}(x)=\psi_{w}(x) e^{-r \sum x_{i}}$, we get

$$
\begin{aligned}
& \int_{\mathbb{R}^{n}} d x \psi_{w}(x) e^{-r \sum x_{i}} \mathcal{T}_{\alpha_{\circ}, \alpha_{n+1}, \ldots, \alpha_{t}}^{\tau}(x) \\
&= e^{-\tau \sum_{i=1}^{n}\left(w_{i}+\mathbf{i} r\right)^{2} / 2} \\
& \quad \times \prod_{i=1}^{n}\left(\Gamma\left(\alpha_{\circ}-\mathbf{i} w_{i}+r\right) \prod_{j=n+1}^{t} \Gamma\left(\alpha_{j}-\mathbf{i} w_{i}+r\right)\right)
\end{aligned}
$$




$$
\times \prod_{1 \leqslant i<j \leqslant n} \Gamma\left(-\mathbf{i}\left(w_{i}+w_{j}\right)+2 r\right) .
$$

When $w \in(-\mathbb{H})^{n}$, we may act on both sides of (151) with $\overline{\mathbf{B}}_{n}^{u}$. Acting on the right-hand side yields

$$
\begin{aligned}
& \int_{\mathbb{R}^{n}} d \xi \mathfrak{m}_{n}(\xi) u^{\mathbf{i} \sum_{i=1}^{n}-w_{i}+\xi_{i}} \prod_{i, j} \Gamma\left(-\mathbf{i} \xi_{i}+\mathbf{i} w_{j}\right) e^{-\tau \sum_{i=1}^{n}\left(\xi_{i}+\mathbf{i} r\right)^{2} / 2} \\
& \quad \times \prod_{i=1}^{n}\left(\Gamma\left(\alpha_{\circ}-\mathbf{i} \xi_{i}+r\right) \prod_{j=n+1}^{t} \Gamma\left(\alpha_{j}-\mathbf{i} \xi_{i}+r\right)\right) \\
& \quad \times \prod_{1 \leqslant i<j \leqslant n} \Gamma\left(-\mathbf{i}\left(\xi_{i}+\xi_{j}\right)+2 r\right) .
\end{aligned}
$$

Acting on the left-hand side with $\overline{\mathbf{B}}_{n}^{u}$ yields

$$
\int_{\mathbb{R}^{n}} d x e^{-u e^{x_{1}}} \psi_{w}(x) e^{-r \sum x_{i}} \mathcal{T}_{\alpha_{o}, \alpha_{n+1}, \ldots, \alpha_{t}}^{\tau}(x) .
$$

We have exchanged the action of $\overline{\mathbf{B}}_{n}^{u}$ and the integration above using the Fubini theorem. It can be applied here because both $e^{-u e^{x_{1}}} \psi_{w}(x)$ and $e^{-r \sum x_{i}} \mathcal{T}_{\alpha_{0}, \alpha_{n+1}, \ldots, \alpha_{t}}^{\tau}(x)$ are bounded in $\mathbb{L}^{2}\left(\mathbb{R}^{n}\right)$, which has been proved respectively in [OSZ14, Corollary 3.8] and Proposition 6.15 of the present paper. We may substitute $w_{i}-\mathbf{i} r$ in place of $w_{i}$, and after a change of variables $z_{i}=\mathbf{i} \xi_{i}-r$, we obtain that

$$
\begin{array}{rl}
\int_{\mathbb{R}^{n}} & d x e^{-u e^{x_{1}}} \psi_{w}(x) \mathcal{T}_{\alpha_{\circ}, \alpha_{n+1}, \ldots, \alpha_{t}}^{\tau}(x) \\
= & \int_{\left(\mathcal{D}_{-r}\right)^{n}}-d \mathbf{i} z \mathfrak{m}_{n}(\mathbf{i} z) u^{\sum_{i=1}^{n}-\mathbf{i} w_{i}+\sum_{i=1}^{n} z_{i}} \\
& \times e^{\tau \sum_{i=1}^{n} z_{i}^{2}} \prod_{i, j=1}^{n} \Gamma\left(-z_{i}+\mathbf{i} w_{j}\right) \prod_{i=1}^{n}\left(\Gamma\left(\alpha_{\circ}-z_{i}\right) \prod_{j=n+1}^{t} \Gamma\left(\alpha_{j}-z_{i}\right)\right) \\
& \times \prod_{1 \leqslant i<j \leqslant n} \Gamma\left(-z_{i}-z_{j}\right) .
\end{array}
$$

The expectation $\mathbb{E}\left[e^{-u e^{T_{1}}}\right]$ under the Whittaker measure $\mathbb{P}_{\left(\alpha_{1}, \ldots, \alpha_{n}\right),\left(\alpha_{0}, \alpha_{n+1}, \ldots, \alpha_{t}, \tau\right)}$ corresponds to the left-hand side of (152) for $w=\left(\mathbf{i} \alpha_{1}, \ldots, \mathbf{i} \alpha_{n}\right)$, divided by the normalization constant, so that we have established (149). For this choice of $w$, we need that $r>\alpha_{i}$ so that $w_{i}+\mathbf{i} r \in \mathbb{H}$ for all $i$, hence the hypothesis in the statement of Theorem 6.32 . 
We turn now to the proof of (150). Again we start from (151) where now $w \in \mathbb{H}^{n}$. Acting on both sides with $\mathbf{B}_{n}^{u}$ (the interchange of integration with respect to $x$ and action of $\mathbf{B}_{n}^{u}$ can be justified as in the previous case) yields for all $w \in \mathbb{H}^{n}$

$$
\begin{aligned}
\int_{\mathbb{R}^{n}} d x e^{-u e^{-x_{n}}} \psi_{w}(x) e^{-r \sum x_{i}} \mathcal{T}_{\alpha_{\circ}, \alpha_{n+1}, \ldots, \alpha_{t}}^{\tau}(x) \\
=\int_{\mathbb{R}^{n}} d \xi \mathfrak{m}_{n}(\xi) u^{\mathbf{i} \sum_{i=1}^{n} w_{i}+\xi_{i}} \prod_{i, j} \Gamma\left(-\mathbf{i} \xi_{i}-\mathbf{i} w_{j}\right) \times e^{-\tau \sum_{i=1}^{n}\left(-\xi_{i}+\mathbf{i} r\right)^{2} / 2} \\
\quad \times \prod_{i=1}^{n}\left(\Gamma\left(\alpha_{\circ}+\mathbf{i} \xi_{i}+r\right) \prod_{j=n+1}^{t} \Gamma\left(\alpha_{j}+\mathbf{i} \xi_{i}+r\right)\right) \\
\quad \times \prod_{1 \leqslant i<j \leqslant n} \Gamma\left(\mathbf{i}\left(\xi_{i}+\xi_{j}\right)+2 r\right) .
\end{aligned}
$$

Now we may substitute back $w_{i}$ by $w_{i}-\mathbf{i} r$ (with $r$ not too large so that $w_{i}-\mathbf{i} r \in$ $\mathbb{H})$, evaluate for $w=\left(\mathbf{i} \alpha_{1}, \ldots, \mathbf{i} \alpha_{n}\right)$, and after a change of variables $z_{i}=\mathbf{i} \xi_{i}+r$, we obtain

$$
\begin{aligned}
\int_{\mathbb{R}^{n}} d x e^{-u e^{-x_{n}}} \psi_{\mathbf{i} \alpha_{1}, \ldots, \mathbf{i} \alpha_{n}}(x) \mathcal{T}_{\alpha_{o}, \alpha_{n+1}, \ldots, \alpha_{t}}^{\tau}(x) \\
=\int_{\left(\mathcal{D}_{r}\right)^{n}} d z \frac{\mathfrak{m}_{n}(\mathbf{i} z)}{\mathbf{i}} u^{\sum_{i=1}^{n} \alpha_{i}+\sum_{i=1}^{n} z_{i}} \\
\quad \times e^{\tau \sum_{i=1}^{n} z_{i}^{2}} \prod_{i, j=1}^{n} \Gamma\left(-z_{i}+\alpha_{j}\right) \prod_{i=1}^{n}\left(\Gamma\left(\alpha_{\circ}+z_{i}\right) \prod_{j=n+1}^{t} \Gamma\left(\alpha_{j}+z_{i}\right)\right) \\
\quad \times \prod_{1 \leqslant i<j \leqslant n} \Gamma\left(z_{i}+z_{j}\right) .
\end{aligned}
$$

We obtain (150) after dividing by the normalization constant.

REMARK 6.39. We expect that one could deduce Theorem 6.38 from Corollaries 6.20 and 6.21. The correspondence between the two types of formulas should follow the same lines as in [BCR13, Section 3], where the equivalence between an $n$-fold contour integral such as (149) and series expansions as in Corollary 6.20 is explained in the context of the full-space log-gamma polymer. Alternatively, we may prove $n$-fold contour integral formulas for the $(q, t)$-Laplace transform of general Macdonald measures by keeping the contour as $\mathcal{D}_{-\varepsilon}$ in the proof of Proposition 3.30. Such formulas would degenerate to Theorem 6.38 in the Whittaker limit.

Note that in the $n$-fold Laplace transform formulas (149) and (150), we may 
let $\tau$ go to zero without encountering any singularity. As a consequence, we can prove a Laplace transform formula for the partition function of the half-space log-gamma polymer (without Plancherel specialization).

Corollary 6.40. Fix $n \leqslant t, \alpha_{1}, \ldots, \alpha_{t}>0$ and $\alpha_{\circ} \in \mathbb{R}$ such that $\alpha_{\circ}+\alpha_{i}>0$ for $1 \leqslant i \leqslant n$. Let $r>0$ such that for all $1 \leqslant i \leqslant n, r>\alpha_{i}>0$. The partition function $Z(t, n)$ of the log-gamma polymer in a half-quadrant (Definition 1.1) is characterized by the following. For any $u>0$,

$$
\begin{aligned}
\mathbb{E}\left[e^{-u Z(t, n)}\right] & \frac{1}{n !} \int_{\mathcal{D}_{r}} \frac{d z_{1}}{2 \mathbf{i} \pi} \cdots \int_{\mathcal{D}_{r}} \frac{d z_{n}}{2 \mathbf{i} \pi} \prod_{i \neq j} \frac{1}{\Gamma\left(z_{i}-z_{j}\right)} \prod_{1 \leqslant i<j \leqslant n} \frac{\Gamma\left(z_{i}+z_{j}\right)}{\Gamma\left(\alpha_{i}+\alpha_{j}\right)} \\
& \times \prod_{i, j=1}^{n} \Gamma\left(z_{i}-\alpha_{j}\right) \prod_{i=1}^{n}\left(u^{\alpha_{i}-z_{i}} \frac{\Gamma\left(\alpha_{\circ}+z_{i}\right)}{\Gamma\left(\alpha_{\circ}+\alpha_{i}\right)} \prod_{j=n+1}^{t} \frac{\Gamma\left(\alpha_{j}+z_{i}\right)}{\Gamma\left(\alpha_{j}+\alpha_{i}\right)}\right) .
\end{aligned}
$$

Proof. We can compute $\mathbb{E}\left[e^{-u Z(t, n, \tau)}\right]$, where $Z(t, n, \tau)$ is the hybrid partition function from Definition 6.33, using the Laplace transform formula from Theorem 6.38 and the identity in law from Proposition 6.34. Then, using the weak convergence $Z(t, n, \tau) \Rightarrow Z(t, n)$ as $\tau \rightarrow 0$, we obtain

$$
\begin{aligned}
\mathbb{E}\left[e^{-u Z(t, n)}\right] & \mathbf{i}^{n} \int_{\left(\mathcal{D}_{-r}\right)^{n}} \frac{\mathfrak{m}_{n}(\mathbf{i} z)}{\mathbf{i}} d z u^{\sum_{i=1}^{n} \alpha_{i}+\sum_{i=1}^{n} z_{i}} \prod_{i, j=1}^{n} \Gamma\left(-z_{i}-\alpha_{j}\right) \\
& \times \prod_{i=1}^{n}\left(\frac{\Gamma\left(\alpha_{\circ}-z_{i}\right)}{\Gamma\left(\alpha_{\circ}+\alpha_{i}\right)} \prod_{j=n+1}^{t} \frac{\Gamma\left(\alpha_{j}-z_{i}\right)}{\Gamma\left(\alpha_{j}+\alpha_{i}\right)}\right) \prod_{1 \leqslant i<j \leqslant n} \frac{\Gamma\left(-z_{i}-z_{j}\right)}{\Gamma\left(\alpha_{i}+\alpha_{j}\right)}
\end{aligned}
$$

which can be written equivalently as in the statement of the Proposition.

\subsection{Plancherel theory and comparison with O'Connell-Seppäläinen-}

Zygouras's results. In this section, we shall compare our results, in particular Corollary 6.40, with formal computations in [OSZ14]. The integral transform

$$
\widehat{f}(\xi)=\int_{\mathbb{R}^{n}} f(x) \psi_{\xi}(x) d x
$$

defines an isometry from $\mathbb{L}^{2}\left(\mathbb{R}^{n}, d x\right)$ to $\mathbb{L}^{2}\left(\mathbb{R}^{n}, \mathfrak{m}_{n}(\xi) d \xi\right)$ restricted to symmetric functions. The associated Plancherel theorem [STS93, Theorem 
51] allows us to compute the Laplace transform of observables of the Whittaker measure [OSZ14]. Indeed, we are interested in computing

$$
\mathbb{E}\left[e^{-u Z(n, n)}\right]=\frac{1}{C} \int_{\mathbb{R}^{n}} e^{-u e^{T_{1}}} \psi_{\mathbf{i} \alpha_{1}, \ldots, \mathbf{i} \alpha_{n}}(T) e^{-\alpha_{\circ} \sum_{i=1}^{n / 2} T_{2 i-1}-T_{2 i}} e^{-e^{-T_{n}}} d T,
$$

where $C$ is the normalizing constant and we assume that $n$ is even for simplicity. Suppose that the integrand above can be written as $\overline{f(T)} g(T)$, where $f$ and $g$ are two functions in $\mathbb{L}^{2}\left(\mathbb{R}^{n}, d x\right)$ such that we are able to compute the integral transforms $\widehat{f}, \widehat{g}$. Then the Plancherel theorem yields

$$
\mathbb{E}\left[e^{-u Z(n, n)}\right]=\frac{1}{C} \int_{\mathbb{R}^{n}} \widehat{f}(\xi) \widehat{g}(\xi) \mathfrak{m}_{n}(\xi) d \xi .
$$

However, it is not clear how to find such a decomposition of the integrand in (155). In [OSZ14, Section 5], in a remark titled 'A formal computation', the authors propose to apply formally this scheme to functions that are not square integrable, in order to derive explicit integral formulas for $\mathbb{E}\left[e^{-u Z(n, n)}\right]$. There may be several ways to decompose the integrand in (155) into a product of functions whose integral transforms can be computed. We examine below two possibilities: the first one yields our formula from Corollary 6.40, while the second one yields [OSZ14, (5.15)]. Although the argument is formal, both approaches lead to the correct answer (though the form of the answer is different and we match them in Corollary 6.41).

6.8.1. Case 1: equivalent to using the operator $\overline{\mathbf{B}}_{n}^{u}$. Let us choose $f$ and $g$ as

$$
f(T)=e^{-u e^{T_{1}}} \psi_{\mathbf{i} \alpha_{1}, \ldots, \mathbf{i} \alpha_{n}}(T), \quad g(T)=e^{-\alpha_{\circ} \sum_{i=1}^{n / 2} T_{2 i-1}-T_{2 i}} e^{-e^{-T_{n}}} .
$$

Then, using (103),

$$
\widehat{f}(\xi)=u^{\sum_{j=1}^{n} \alpha_{j}-\mathbf{i} \xi_{j}} \prod_{1 \leqslant i, j \leqslant n} \Gamma\left(\mathbf{i} \xi_{j}-\alpha_{i}\right), \quad \mathfrak{R e}\left[\mathbf{i} \xi_{i}\right]>\alpha_{j} \quad \text { for all } 1 \leqslant i, j \leqslant n,
$$

and using Theorem 6.32 (that is [OSZ14, Corollary 5.4]),

$$
\widehat{g}(\xi)=\prod_{j=1}^{n} \Gamma\left(\alpha_{\circ}-\mathbf{i} \xi_{j}\right) \prod_{1 \leqslant i<j \leqslant n} \Gamma\left(-\mathbf{i} \xi_{i}-\mathbf{i} \xi_{j}\right),
$$

where $\mathfrak{R e}\left[-\mathbf{i} \xi_{i}\right]>\min \left\{0,-\alpha_{\circ}\right\}$ for all $1 \leqslant i \leqslant n$. Thus a formal application of the Plancherel theorem suggests that

$$
\mathbb{E}\left[e^{-u Z(n, n)}\right]=\int u^{\sum_{j=1}^{n} \alpha_{j}-\mathbf{i} \xi_{j}} \prod_{1 \leqslant i, j \leqslant n} \Gamma\left(\mathbf{i} \xi_{j}-\alpha_{i}\right) \prod_{j=1}^{n} \frac{\Gamma\left(\alpha_{\circ}+\mathbf{i} \xi_{j}\right)}{\Gamma \alpha_{\circ}+\alpha_{j}}
$$




$$
\times \prod_{1 \leqslant i<j \leqslant n} \frac{\Gamma\left(\mathbf{i} \xi_{i}+\mathbf{i} \xi_{j}\right)}{\Gamma\left(\alpha_{i}+\alpha_{j}\right)} \mathfrak{m}_{n}(\xi) d \xi
$$

where the integration contour is $\mathbb{R}-\mathbf{i} r$ with $r>\alpha_{i}$ for all $1 \leqslant i \leqslant n$. This formula is, in fact, correct as we have proved it in Corollary 6.40 (in the special case $t=n$ ), itself coming from Theorem 6.38. The choice of contours can be justified more precisely by shifting variables as in the proof of Theorem 6.38 . However, the derivation above is not rigorous due to the fact that the functions $f$ and $g$ do not belong to $\mathbb{L}^{2}\left(\mathbb{R}^{n}, d x\right)$. This is why the presence of the Plancherel component in the proof of Theorem 6.38 is crucial.

6.8.2. Case 2: O'Connell-Seppäläinen-Zygouras's approach. Let us now choose $f$ and $g$ as

$$
f(T)=e^{-e^{-T_{n}}} \psi_{\mathbf{i} \alpha_{1}, \ldots, \mathbf{i} \alpha_{n}}(T), \quad g(T)=e^{-\alpha_{\circ} \sum_{i=1}^{n / 2} T_{2 i-1}-T_{2 i}} e^{-u e^{T_{1}}} .
$$

Then, using (102),

$$
\widehat{f}(\xi)=\prod_{1 \leqslant i, j \leqslant n} \Gamma\left(\mathbf{i} \xi_{j}-\alpha_{i}\right), \quad \mathfrak{R e}\left[\mathbf{i} \xi_{j}\right]>\alpha_{i} \quad \text { for all } 1 \leqslant i, j \leqslant n .
$$

Using $\psi_{z}\left(T_{1}, \ldots, T_{n}\right)=\psi_{-z}\left(-T_{n}, \ldots,-T_{1}\right)$ we have that for $n$ even,

$$
\widehat{g}(\xi)=\int_{\mathbb{R}^{n}} e^{-\alpha_{\circ} \sum_{i=1}^{n / 2} T_{2 i-1}-T_{2 i}} e^{-u e^{-T_{n}}} \psi_{-\xi}(T) d T
$$

so that using [OSZ14, Corollary 5.4]),

$$
\widehat{g}(\xi)=\prod_{j=1}^{n} \Gamma\left(\alpha_{\circ}+\mathbf{i} \xi_{j}\right) \prod_{1 \leqslant i<j \leqslant n} \Gamma\left(\mathbf{i} \xi_{i}+\mathbf{i} \xi_{j}\right) u^{-\sum_{j=1}^{n} \mathbf{i} \xi_{j}},
$$

where $\mathfrak{R e}\left[\mathbf{i} \xi_{j}\right]>\min \left\{0,-\alpha_{\circ}\right\}$ for all $1 \leqslant j \leqslant n$. Thus the Plancherel theorem suggests that

$$
\begin{aligned}
\mathbb{E}\left[e^{-u Z(n, n)}\right]= & \int_{\mathbb{R}^{n}} u^{-\sum_{j=1}^{n} \mathbf{i} \xi_{j}} \prod_{1 \leqslant i, j \leqslant n} \Gamma\left(\mathbf{i} \xi_{j}+\alpha_{i}\right) \prod_{j=1}^{n} \frac{\Gamma\left(\alpha_{\circ}+\mathbf{i} \xi_{j}\right)}{\Gamma\left(\alpha_{\circ}+\alpha_{j}\right)} \\
& \times \prod_{1 \leqslant i<j \leqslant n} \frac{\Gamma\left(\mathbf{i} \xi_{i}+\mathbf{i} \xi_{j}\right)}{\Gamma\left(\alpha_{i}+\alpha_{j}\right)} \mathfrak{m}_{n}(\xi) d \xi,
\end{aligned}
$$

where the contour is $\mathbb{R}-\mathbf{i} r$ with $r>\min \left\{0,-\alpha_{\circ}\right\}$. Again, the choice of contour could be justified more precisely by shifting variables, but the derivation above 
is not rigorous because the function $g$ does not belong to $\mathbb{L}^{2}\left(\mathbb{R}^{n}, d x\right)$. Since our partition function $Z(n, n)$ has the same distribution as the random variable $2 t_{n n}$ under the measure $\tilde{v}_{\alpha, \zeta}(d w)$ as defined in [OSZ14, (5.10)], expression (157) is exactly equivalent to [OSZ14, (5.15)] (after making a change of variables $z_{i}=\mathbf{i} \xi_{i}$ and indentifying $u=r / 2$ ). A similar argument can be adapted in the case where $n$ is odd.

Note that (156) and (157) seem to be quite different. There is an additional factor $u^{\sum_{j=1}^{n} \alpha_{j}}$ in (156), and the factor $\prod_{1 \leqslant i, j \leqslant n} \Gamma\left(\mathbf{i} \xi_{j}-\alpha_{i}\right)$ in (156) becomes $\prod_{1 \leqslant i, j \leqslant n} \Gamma\left(\mathbf{i} \xi_{j}+\alpha_{i}\right)$ in (157). The next corollary shows that (157) (or equivalently (158) below after a change of variables) can be proved as well from Corollary 6.40.

Corollary 6.41. Fix $\alpha_{1}, \ldots, \alpha_{n}>0$ and $\alpha_{\circ} \in \mathbb{R}$ such that $\alpha_{\circ}+\alpha_{i}>0$ for $1 \leqslant i \leqslant n$. Then, when $n$ is even, for any $u>0$,

$$
\begin{aligned}
\mathbb{E}\left[e^{-u Z(n, n)}\right] & =\frac{1}{n !} \int_{\mathcal{D}_{r}} \frac{d z_{1}}{2 \mathbf{i} \pi} \cdots \int_{\mathcal{D}_{r}} \frac{d z_{n}}{2 \mathbf{i} \pi} \prod_{i \neq j} \frac{1}{\Gamma\left(z_{i}-z_{j}\right)} \\
& \times \prod_{1 \leqslant i<j \leqslant n} \frac{\Gamma\left(z_{i}+z_{j}\right)}{\Gamma\left(\alpha_{i}+\alpha_{j}\right)} \prod_{i, j=1}^{n} \Gamma\left(z_{i}+\alpha_{j}\right) \prod_{i=1}^{n} u^{-z_{i}} \frac{\Gamma\left(\alpha_{\circ}+z_{i}\right)}{\Gamma\left(\alpha_{\circ}+\alpha_{i}\right)},
\end{aligned}
$$

where $r>0$ is such that $r+\alpha_{\circ}>0$.

When $n$ is odd, for any $u>0$,

$$
\begin{aligned}
\mathbb{E}\left[e^{-u Z(n, n)}\right]= & \frac{u^{\alpha_{\circ}}}{n !} \int_{\mathcal{D}_{r}} \frac{d z_{1}}{2 \mathbf{i} \pi} \cdots \int_{\mathcal{D}_{r}} \frac{d z_{n}}{2 \mathbf{i} \pi} \prod_{i \neq j} \frac{1}{\Gamma\left(z_{i}-z_{j}\right)} \\
& \times \prod_{1 \leqslant i<j \leqslant n} \Gamma\left(z_{i}+z_{j}\right) \prod_{i, j=1}^{n} \Gamma\left(z_{i}+\alpha_{j}\right) \prod_{i=1}^{n} u^{-z_{i}} \Gamma\left(z_{i}-\alpha_{\circ}\right),
\end{aligned}
$$

where $r>0$ is such that $r-\alpha_{\circ}>0$.

REMARK 6.42. Equation (158) corresponds exactly to [OSZ14, (5.15)] given that $2 t_{n n}$ in $[$ OSZ14, (5.15)] has the same distribution as our partition function $Z(n, n)$. In the case where $n$ is odd, our formula (159) is slightly different from [OSZ14, (5.16)]; we find an extra factor $u^{\alpha_{\circ}}$. We believe that this is due to a typo in [OSZ14, (5.16)] (otherwise, the case $n=1$ would be in contradiction with [OSZ14, Corollary 3.9]). 
Proof. Consider the functions

$$
f_{\alpha_{\circ}}\left(\alpha_{1}, \ldots, \alpha_{n}\right)=\int_{\mathbb{R}^{n}} e^{-u e^{T_{1}}} \psi_{\mathbf{i} \alpha}(T) e^{-\alpha_{\circ} \sum_{i=1}^{n / 2}\left(T_{2 i-1}-T_{2 i}\right)} e^{-e^{-T_{n}}} d T
$$

and

$$
\begin{aligned}
& g_{\alpha_{\circ}}\left(\alpha_{1}, \ldots, \alpha_{n}\right) \\
& \quad=\frac{1}{n !} \int_{\mathcal{D}_{r}} \frac{d z_{1}}{2 \mathbf{i} \pi} \cdots \int_{\mathcal{D}_{r}} \frac{d z_{n}}{2 \mathbf{i} \pi} \prod_{i \neq j} \frac{1}{\Gamma\left(z_{i}-z_{j}\right)} \\
& \quad \times \prod_{1 \leqslant i<j \leqslant n} \Gamma\left(z_{i}+z_{j}\right) \prod_{i, j=1}^{n} \Gamma\left(z_{i}-\alpha_{j}\right) \prod_{i=1}^{n} u^{\alpha_{i}-z_{i}} \Gamma\left(\alpha_{\circ}+z_{i}\right),
\end{aligned}
$$

where the contour $\mathcal{D}_{r}$ is such that $r>\alpha_{i}$ for all $1 \leqslant i \leqslant n$ (the contour can be freely shifted to the right so that $g_{\alpha_{0}}\left(\alpha_{1}, \ldots, \alpha_{n}\right)$ does not depend on $r$ ). We know from Corollary 6.40 that for $\alpha \in\left(\mathbb{R}_{>0}\right)^{n}$ with $\alpha_{i}+\alpha_{\circ}>0$,

$$
f_{\alpha_{\circ}}\left(\alpha_{1}, \ldots, \alpha_{n}\right)=g_{\alpha_{\circ}}\left(\alpha_{1}, \ldots, \alpha_{n}\right) .
$$

We will need to analytically continue this relation to negative values of $\alpha_{i}$. The integral in (161) is analytic in each variable $\alpha_{i}$ as long as $\mathfrak{R e}\left[\alpha_{i}\right]<r$, and $r$ can be taken arbitrarily large so that $g_{\alpha_{0}}\left(\alpha_{1}, \ldots, \alpha_{n}\right)$ is analytic in each variable $\alpha_{i}$ on $\mathbb{R}$. Before proving that $f_{\alpha_{0}}\left(\alpha_{1}, \ldots, \alpha_{n}\right)$ is analytic as well, let us see how this implies Corollary 6.41.

Using the change of variables $T_{i}=-\log (u)-\tilde{T}_{n-i+1}$ for all $1 \leqslant i \leqslant n$, we obtain that when $n$ is even

$$
\begin{aligned}
f_{\alpha_{\circ}}\left(\alpha_{1}, \ldots, \alpha_{n}\right) & =\int_{\mathbb{R}^{n}} e^{-e^{-\widetilde{T}_{n}}} \psi_{-\mathbf{i} \alpha}(\widetilde{T}+\log (u)) e^{-\alpha_{\circ} \sum_{i=1}^{n / 2} \widetilde{T}_{2 i-1}-\widetilde{T}_{2 i}} e^{-u e^{\widetilde{T}_{1}}} d \widetilde{T} \\
& =u^{\sum_{i=1}^{n} \alpha_{i}} f_{\alpha_{\circ}}\left(-\alpha_{1}, \ldots,-\alpha_{n}\right) .
\end{aligned}
$$

Similarly, when $n$ is odd,

$$
\begin{aligned}
f_{\alpha_{\circ}}\left(\alpha_{1}, \ldots, \alpha_{n}\right) & =\int_{\mathbb{R}^{n}} e^{-e^{-\widetilde{T}_{n}}} \psi_{-\mathbf{i} \alpha}(\widetilde{T}+\log (u)) e^{+\alpha_{\circ} \sum_{i=1}^{n / 2} \widetilde{T}_{2 i-1}-\widetilde{T}_{2 i}} e^{\alpha_{\circ} \log (u)} e^{-u e^{\widetilde{T}_{1}}} d \widetilde{T} \\
& =u^{\alpha_{\circ}+\sum_{i=1}^{n} \alpha_{i}} f_{-\alpha_{\circ}}\left(-\alpha_{1}, \ldots,-\alpha_{n}\right) .
\end{aligned}
$$

Using (162) and the equality $f_{\alpha_{\circ}}\left(-\alpha_{1}, \ldots,-\alpha_{n}\right)=g_{\alpha_{\circ}}\left(-\alpha_{1}, \ldots,-\alpha_{n}\right)$ for $\alpha_{i} \in \mathbb{R}$, we find when $n$ is even

$$
f_{\alpha_{\circ}}\left(\alpha_{1}, \ldots, \alpha_{n}\right)=\frac{1}{n !} \int_{\mathcal{D}_{r}} \frac{d z_{1}}{2 \mathbf{i} \pi} \cdots \int_{\mathcal{D}_{r}} \frac{d z_{n}}{2 \mathbf{i} \pi} \prod_{i \neq j} \frac{1}{\Gamma\left(z_{i}-z_{j}\right)}
$$




$$
\times \prod_{1 \leqslant i<j \leqslant n} \Gamma\left(z_{i}+z_{j}\right) \prod_{i, j=1}^{n} \Gamma\left(z_{i}+\alpha_{j}\right) \prod_{i=1}^{n} u^{-z_{i}} \Gamma\left(\alpha_{\circ}+z_{i}\right) .
$$

We can finally freely move the contour as long as the real part stays positive to arrive at the statement of Corollary 6.41. Similarly, using (163) when $n$ is odd,

$$
\begin{aligned}
f_{\alpha_{\circ}}\left(\alpha_{1}, \ldots, \alpha_{n}\right)= & u^{\alpha_{\circ}} \frac{1}{n !} \int_{\mathcal{D}_{r}} \frac{d z_{1}}{2 \mathbf{i} \pi} \cdots \int_{\mathcal{D}_{r}} \frac{d z_{n}}{2 \mathbf{i} \pi} \prod_{i \neq j} \frac{1}{\Gamma\left(z_{i}-z_{j}\right)} \\
& \times \prod_{1 \leqslant i<j \leqslant n} \Gamma\left(z_{i}+z_{j}\right) \prod_{i, j=1}^{n} \Gamma\left(z_{i}+\alpha_{j}\right) \prod_{i=1}^{n} u^{-z_{i}} \Gamma\left(-\alpha_{\circ}+z_{i}\right),
\end{aligned}
$$

where the contour $\mathcal{D}_{r}$ is such that $r-\alpha_{\circ}>0$.

Now we turn to proving analyticity of $f_{\alpha_{0}}\left(\alpha_{1}, \ldots, \alpha_{n}\right)$ via the next two lemmas.

Lemma 6.43. For any $\alpha_{\circ}, \alpha_{1}, \ldots, \alpha_{n} \in \mathbb{R}, u>0$, we have

$$
\begin{aligned}
f_{\alpha_{\circ}}\left(\alpha_{1}, \ldots, \alpha_{n}\right)= & \int_{\mathbb{R}^{\frac{n(n+1)}{2}}} e^{-u \sum_{\pi} \prod_{(i, j) \in \pi} e^{x_{i j}}} \prod_{1 \leqslant i<j \leqslant n} e^{-\left(\alpha_{i}+\alpha_{j}\right) x_{i j}} e^{-e^{-x_{i j}}} \\
& \times \prod_{1 \leqslant i \leqslant n} e^{-\left(\alpha_{i}+\alpha_{\circ}\right) x_{i i}} e^{-e^{-x_{i i}}} d x
\end{aligned}
$$

where the sum over $\pi$ in the first exponential term is a sum over all up-right paths from $(1,1)$ to $(n, n)$ in the lower half-quadrant.

Proof. Note that for $\alpha \in\left(\mathbb{R}_{>0}\right)^{n}, f_{\alpha_{\circ}}\left(\alpha_{1}, \ldots, \alpha_{n}\right)$ is the unnormalized Laplace transform of $e^{T_{1}}$ under the half-space Whittaker measure; it is also the unnormalized Laplace transform of the polymer partition function, which can be written as in (164). More generally, for any $\alpha_{\circ}, \alpha_{1}, \ldots, \alpha_{n} \in \mathbb{R},(164)$ is obtained from (160) via a change of variables corresponding to the geometric RSK map. This map is volume preserving [OSZ14, Theorem 3.1]. It is shown in [OSZ14, (3.8) and Corollary 3.3] how the integrand in (160) becomes the integrand in (164) via this change of variables.

LeMMA 6.44. For any $\alpha_{\circ} \in \mathbb{R}$ and $u>0, f_{\alpha_{\circ}}\left(\alpha_{1}, \ldots, \alpha_{n}\right)$ is analytic in each variable $\alpha_{i}$ on $\mathbb{R}$.

Proof. We show that for $\alpha=\left(\alpha_{1}, \ldots, \alpha_{n}\right)$ in a compact subset of $\mathbb{R}^{n}$, the integral in (164) is absolutely convergent uniformly in $\alpha$. For $\alpha$ in a compact set, we may 
rewrite (164) as

$$
\begin{aligned}
& f_{\alpha_{\circ}}\left(\alpha_{1}, \ldots, \alpha_{n}\right) \\
& \quad=\int_{\mathbb{R}^{\frac{n(n+1)}{2}}} C_{\alpha}(x) \exp \left(c \sum_{i, j}\left|x_{i, j}\right|-\sum_{\pi}\left(u e^{\sum_{(i, j) \in \pi} x_{i, j}}+\sum_{(i, j) \in \pi} c_{i, j} e^{-x_{i, j}}\right)\right) d x,
\end{aligned}
$$

where we choose the constant $c$ large enough so that the prefactor $C_{\alpha}(x)$ is an integrable function over $\mathbb{R}^{\frac{n(n+1)}{2}}$ (uniformly for $\alpha$ in a compact set), and for each $i, j$, the constant $c_{i, j}$ is the inverse of the number of paths containing the vertex $(i, j)$. In order to prove uniform integrability, it is enough to show that each term in the sum over paths $\pi$ grows exponentially, thus compensating the term $c \sum_{i, j}\left|x_{i, j}\right|$. More precisely, we need to show that there exist some constants $k, k^{\prime}$ such that for $x \notin[-M, M]^{\frac{n(n+1)}{2}}$ and any path $\pi$ of fixed length,

$$
u e^{\sum_{(i, j) \in \pi} x_{i, j}}+\sum_{(i, j) \in \pi} c_{i, j} e^{-x_{i, j}} \geqslant k e^{k^{\prime} M} .
$$

In order to prove this, we show that for any constant $c^{\prime}>0$, the auxiliary function $g\left(x_{1}, \ldots, x_{m}\right)=c^{\prime} e^{\sum_{i=1}^{m} x_{i}}+\sum_{i=1}^{m} e^{-x_{i}}$ satisfies the same bound. Indeed, take $x \in \mathbb{R}^{m} \backslash[-M, M]^{m}$; either there exists some $i$ for which $x_{i}<-M$ or we have $x_{i}>M$ for all $i$. In the first case, $g\left(x_{1}, \ldots, x_{m}\right)>e^{M}$ and in the second case $g\left(x_{1}, \ldots, x_{m}\right)>c^{\prime} e^{m M}$. This concludes the proof of the lemma.

\section{KPZ equation on the half-line}

7.1. KPZ equation on $\mathbb{R}_{\geqslant 0}$. The KPZ equation on $\mathbb{R}_{\geqslant 0}$ with the Neumann boundary condition is the a priori ill-posed stochastic PDE

$$
\left\{\begin{array}{l}
\partial_{T} \mathcal{H}=\frac{1}{2} \Delta \mathcal{H}+\frac{1}{2}\left(\partial_{X} \mathcal{H}\right)^{2}+\dot{W}, \\
\left.\partial_{X} \mathcal{H}(T, X)\right|_{X=0}=A \quad(\forall T>0),
\end{array}\right.
$$

where $W$ is a space-time white noise. Following [CS18], we say that $\mathcal{H}$ solves this equation in the Cole-Hopf sense with the narrow-wedge initial condition when $\mathcal{H}=\log \mathcal{Z}$ and $\mathcal{Z}$ is a mild solution to the multiplicative stochastic heat equation (SHE) with the Robin boundary condition

$$
\left\{\begin{array}{l}
\partial_{T} \mathcal{Z}=\frac{1}{2} \Delta \mathcal{Z}+\mathcal{Z} \dot{W} \\
\left.\partial_{X} \mathcal{Z}(T, X)\right|_{X=0}=A \mathcal{Z}(T, 0) \quad(\forall T>0),
\end{array}\right.
$$


with the delta initial condition. More precisely, a mild solution to (166) solves

$$
\mathcal{Z}(T, X)=\mathcal{P}_{T}^{R}(X, 0)+\int_{0}^{T} \int_{0}^{\infty} \mathcal{P}_{T-S}^{R}(X, Y) \mathcal{Z}(S, Y) d W_{S}(d Y),
$$

understood as an Itô integral, where $\mathcal{Z}(T, \cdot)$ is adapted to the filtration $\sigma\left\{\mathcal{Z}(0, \cdot),\left.W\right|_{[0, T]}\right\}$, and $\mathcal{P}^{R}$ is the heat kernel satisfying the Robin boundary condition for all $T, Y>0$

$$
\left.\partial_{X} \mathcal{P}_{T}^{R}(X, Y)\right|_{X=0}=A \mathcal{P}_{T}^{R}(0, Y)
$$

The KPZ equation and the multiplicative SHE arise as a limit of several stochastic processes in the KPZ universality class, which can be divided into two classes: (1) Systems with a tunable asymmetry, such as ASEP, for which the exponential of the height function is expected to converge to the multiplicative SHE when time and space are rescaled and the asymmetry vanishes simultaneously. For the half-line ASEP, this was proved in [CS18, Par19]. (2) Systems with a temperature, or at least a parameter controlling the strength of the noise, such as directed polymers. The partition function is expected to converge in general to the multiplicative SHE when time and space are rescaled and the temperature is sent to infinity simultaneously. In the full space, convergence of directed polymer partition functions to the multiplicative SHE is proved in [AKQ14]. A half-space analogue is in preparation [Wu18].

In the following, we will see how some of our moment formulas obtained above degenerate in the scaling leading to the solution to the KPZ equation. We will focus on the log-gamma directed polymer in a half-quadrant as the parameters of the Gamma random variables go to infinity.

7.2. Log-gamma polymer at high temperature. Consider the half-space log-gamma polymer partition function $Z(t, n)$ and scale parameters as $t=$ $T n+n^{1 / 2} X, \alpha_{i}=n^{1 / 2}, \alpha_{\circ} \in \mathbb{R}$ stays unscaled. Define the rescaled partition function

$$
\mathcal{Z}_{n}(T, X)=C(T, X, n) Z\left(\frac{T}{2} n+n^{1 / 2} X, \frac{T}{2} n\right),
$$

where the normalization factor is

$$
C(T, X, n)=\exp \left(\frac{T n \log (n)-(T-X \log (n)) n^{1 / 2}}{2}-\frac{T}{8}-\frac{X}{2}\right) .
$$

Proposition 7.1. Let $T>0, X \geqslant 0$ and $\alpha_{\circ} \in \mathbb{R}$. For all $k \in \mathbb{Z}_{>0}$,

$$
\lim _{n \rightarrow \infty} \mathbb{E}\left[\mathcal{Z}_{n}(T, X)^{k}\right]
$$




$$
\begin{aligned}
& =2^{k} \int_{r_{1}-\mathbf{i} \infty}^{r_{1}+\mathbf{i} \infty} \frac{d z_{1}}{2 \mathbf{i} \pi} \cdots \int_{r_{k}-\mathbf{i} \infty}^{r_{k}+\mathbf{i} \infty} \frac{d z_{k}}{2 \mathbf{i} \pi} \\
& \times \prod_{1 \leqslant a<b \leqslant k} \frac{z_{a}-z_{b}}{z_{a}-z_{b}-1} \frac{z_{a}+z_{b}}{z_{a}+z_{b}-1} \\
& \times \prod_{m=1}^{k} \frac{z_{m}}{z_{m}+\alpha_{\circ}-1 / 2} \exp \left(\frac{T}{2} z_{m}^{2}-z_{m} x\right),
\end{aligned}
$$

where $r_{1}>r_{2}+1>\cdots>r_{k}+k-1$ and $r_{k}>-\alpha_{\circ}+1 / 2$.

REMARK 7.2. The right-hand side of (170) coincides with moment formulas for the continuous directed polymer in a half-space obtained in [BBC16, Eq. (2)] using the Bethe ansatz. (It was proved only assuming the uniqueness of solutions for the half-space delta Bose gas evolution equations.) Hence, we deduce that the parameter $\alpha_{\circ}$ in the half-space log-gamma polymer is related to the boundary parameter $A$ in the stochastic PDEs (165) and (166) via $\alpha_{\circ}=A+1 / 2$.

Proof. Fix $k \in \mathbb{Z}_{>0}$. Then for $\alpha_{1}, \ldots, \alpha_{t}>0$ sufficiently large, (146) yields (we have shifted all variables by $1 / 2$ )

$$
\begin{aligned}
& \mathbb{E}\left[Z(t, n)^{k}\right]=\oint \frac{d w_{1}}{2 \mathbf{i} \pi} \cdots \oint \frac{d w_{k}}{2 \mathbf{i} \pi} \prod_{1 \leqslant a<b \leqslant k} \frac{w_{a}-w_{b}}{w_{a}-w_{b}-1} \frac{w_{a}+w_{b}}{w_{a}+w_{b}+1} \\
& \quad \times \prod_{m=1}^{k} \frac{2 w_{m}}{w_{m}+1 / 2-\alpha_{\circ}} \prod_{i=1}^{t}\left(\frac{1}{\alpha_{i}-w_{m}-1 / 2}\right) \prod_{j=1}^{n}\left(\frac{1}{w_{m}-1 / 2+\alpha_{j}}\right),
\end{aligned}
$$

where the contours are such that for all $1 \leqslant c \leqslant k$, the contour for $w_{c}$ encloses $\left\{-\alpha_{j}+1 / 2\right\}_{1 \leqslant j \leqslant n}$ and $\left\{w_{c+1}+1, \ldots, w_{k}+1\right\}$, and excludes the poles of the integrand at $\alpha_{\circ}-1 / 2$ and $\alpha_{j}-1 / 2$ (for $1 \leqslant j \leqslant t$ ). Furthermore, if $t \leqslant n$ are large, there is no pole at infinity and we may assume that the contour for $w_{i}$ is the vertical line from $r_{i}-\mathbf{i} \infty$ to $r_{i}+\mathbf{i} \infty$, where the $r_{i}$ are chosen so that

$$
r_{k}+k-1<\cdots<r_{2}+1<r_{1}<\alpha_{\circ}-1 / 2 \text {. }
$$

For $\alpha_{i} \equiv n^{1 / 2}$

$$
\begin{aligned}
& \prod_{i=1}^{T n / 2+n^{1 / 2} X}\left(\frac{1}{\alpha_{i}-w-1 / 2}\right) \prod_{j=1}^{T n / 2}\left(\frac{1}{w-1 / 2+\alpha_{j}}\right) \\
& =\exp \left(\frac{-T n \log (n)+(T-X \log (n)) n^{1 / 2}}{2}+\frac{T}{8}+\frac{X}{2}+\frac{T}{2} w^{2}+w x+o(1)\right) .
\end{aligned}
$$


Moreover, the convergence holds uniformly in $w$. This is because for any fixed $a \in \mathbb{R}$, the convergence

$$
\left(\frac{1}{1-z / n}\right)^{n} \underset{n \rightarrow \infty}{\longrightarrow} e^{z}
$$

holds uniformly for $z$ in the set $\{z \in \mathbb{C}: \mathfrak{R e}[z]<a\}$. It then suffices to apply this convergence twice, for $z=\frac{T}{2} w^{2}$ and $z=w x$, and observe that along the contours that we have chosen, the real part of $w_{i}$ and $w_{i}^{2}$ stays bounded. Using scaling (169), we obtain that the limit of $\mathbb{E}\left[\mathcal{Z}_{n}(T, X)^{k}\right]$ is

$$
\begin{aligned}
& 2^{k} \int_{r_{1}-\mathbf{i} \infty}^{r_{1}+\mathbf{i} \infty} \frac{d w_{1}}{2 \mathbf{i} \pi} \cdots \int_{r_{k}-\mathbf{i} \infty}^{r_{k}+\mathbf{i} \infty} \frac{d w_{k}}{2 \mathbf{i} \pi} \prod_{1 \leqslant a<b \leqslant k} \frac{w_{a}-w_{b}}{w_{a}-w_{b}-1} \frac{w_{a}+w_{b}}{w_{a}+w_{b}+1} \\
& \quad \times \prod_{m=1}^{k} \frac{w_{m}}{w_{m}+1 / 2-\alpha_{\circ}} \exp \left(\frac{T}{2} w_{m}^{2}+w_{m} x\right) .
\end{aligned}
$$

The final result is obtained by applying the change of variables $w_{i}=-z_{k-i+1}$ for all $i$.

Even if one could identify (170) with the moments of $\mathcal{Z}(T, X)$ (the solution to (166)), this would not determine completely the distribution since the moments grow too fast. In order to fully characterize the distribution of $\mathcal{Z}(T, X)$, we would need to analyze the limit of the Laplace transform formula from Corollary 6.20 under scaling (169). It is not obvious how to take this limit rigorously and we leave this for future consideration.

Note that in the special case where $A=-1 / 2$, the distribution of $\mathcal{Z}(T, 0)$ is fully characterized in [BBCW18, Theorem B] (see also [Par19, Corollary 1.3]), and it would be interesting to compare those formulas with the limit of Corollary 6.20 in the case $\alpha_{\circ}=0$.

\section{Tracy-Widom asymptotics for the log-gamma polymer partition function}

The aim of this section is to explain how to manipulate our Laplace transform formulas in order to derive limit theorems for the partition function of the halfspace log-gamma polymer $Z(n, m)$ in various ranges of parameters. In this derivation, we will perform several nonrigorous steps. Most of them can be made rigorous with some additional technical arguments. However, there is one important obstruction to rigor that we cannot presently overcome: we are unable to show that the infinite series that we manipulate are uniformly summable as $n, m \rightarrow \infty$, which suggests that we miss structural cancellations hidden in the formulas. 
It is reasonable to expect that the fluctuations of the free energy $\log (Z(n, m))$ are of the same nature as the fluctuations of last-passage percolation in a halfquadrant, studied in [BR01b, BBCS18b, BBNV18], which corresponds to the zero-temperature limit. In particular, the fluctuations of $\log (Z(n, m))$ should be the same as in the full-space case when $n \gg m \gg 1$, that is, converge to the Tracy-Widom distribution associated to the Gaussian Unitary Ensemble (GUE). We will focus below on the more interesting case of fluctuations close to the boundary for which we expect a phase transition as the boundary parameter varies.

Consider the half-space log-gamma polymer partition function $Z(n, n)$ as in Definition 1.1 with $\alpha_{1}=\cdots=\alpha_{n}=\alpha>0$. Let us scale $u=-e^{-n f-n^{1 / 3} \sigma x}$, where $f, \sigma$ are constants to be determined later. If we have the pointwise convergence for every $x \in \mathbb{R}$

$$
\mathbb{E}\left[e^{u Z(n, n)}\right] \underset{n \rightarrow \infty}{\longrightarrow} F(x),
$$

where $F(x)$ is the distribution function of a certain probability distribution, then it follows (see [BC14, Lemma 4.1.39]) that

$$
\lim _{n \rightarrow \infty} \mathbb{P}\left(\frac{\log (Z(n, n))-f n}{\sigma n^{1 / 3}} \leqslant x\right)=F(x) .
$$

It is not clear how to take asymptotics from the formula for the Laplace transform of $Z(n, n)$ in Corollary 6.40 or 6.41 . However, we have a formula for the Laplace transform of $Z(n, n, \tau)$ from Corollary 6.20, which seems more adapted to asymptotic analysis. Moreover, $Z(n, n, \tau)$ should be close to $Z(n, n, 0)=$ $Z(n, n)$ for fixed $\tau$ in the sense that $n^{-1 / 3}|\log (Z(n, n, \tau))-\log (Z(n, n, 0))|$ converges to zero in probability as $n$ goes to infinity.

Thus, we are left with studying the asymptotics of $\log (Z(n, n, \tau))$ as $n$ goes to infinity for fixed $\tau$. Using the change of variables $z_{i}=s_{i}-v_{i}$ in the statement of Corollary 6.20, we have

$$
\begin{aligned}
\mathbb{E} & {\left[e^{u Z(n, n, \tau)}\right]=\sum_{k=0}^{n} \frac{1}{k !} \int_{\mathcal{D}_{R}} \frac{d z_{1}}{2 \mathbf{i} \pi} \cdots \int_{\mathcal{D}_{R}} \frac{d z_{k}}{2 \mathbf{i} \pi} \oint \frac{d v_{1}}{2 \mathbf{i} \pi} \cdots \oint \frac{d v_{k}}{2 \mathbf{i} \pi} } \\
& \times \prod_{1 \leqslant i<j \leqslant k} \frac{\left(z_{i}-z_{j}\right)\left(v_{i}-v_{j}\right) \Gamma\left(v_{i}+v_{j}\right) \Gamma\left(-z_{i}-z_{j}\right)}{\left(-v_{i}-z_{j}\right)\left(-v_{j}-z_{i}\right) \Gamma\left(v_{j}-z_{i}\right) \Gamma\left(v_{i}-z_{j}\right)} \prod_{i=1}^{k} \frac{\Gamma\left(2 v_{i}\right)}{\Gamma\left(v_{i}-z_{i}\right)} \\
& \times \prod_{i=1}^{k}\left[\frac{\pi}{\sin \left(\pi\left(v_{i}+z_{i}\right)\right)} e^{n\left(G\left(v_{i}\right)+G\left(z_{i}\right)\right)-n^{1 / 3} x \sigma\left(v_{i}+z_{i}\right)} \frac{\Gamma\left(\alpha_{\circ}-z_{i}\right)}{\Gamma\left(\alpha_{\circ}+v_{i}\right)} \frac{e^{\tau z_{i}^{2} / 2-\tau v_{i}^{2} / 2}}{v_{i}+z_{i}}\right],
\end{aligned}
$$

where the contour for the variables $v_{i}$ is a small positively oriented circle around 
$\alpha$, the contour $\mathcal{D}_{R}=R+\mathbf{i} \mathbb{R}$ is such that $-\alpha<R<\min \left\{0, \alpha_{\circ}, 1-\alpha\right\}$ and

$$
G(z)=\log (\Gamma(\alpha-z))-\log (\Gamma(\alpha+z))-f z .
$$

We may deform the integration contours as long as we do not cross poles. In particular, we must ensure that $\mathfrak{R e}\left[z_{i}+v_{i}\right] \in(0,1)$ and that the poles when $z_{i}=\alpha_{\circ}$ and when $z_{i}=-z_{j}$ lie to the right of the integration contour of $z_{i}$.

The asymptotic behavior as $n$ goes to infinity of expansions such as (171) is usually analyzed using the saddle-point method. One needs to study the function $G(z)$, deform the contours so that they go through a critical point of $G$ and justify that the main asymptotic contributions of the integral is localized in a neighborhood of this critical point, where one may use straightforward approximations.

Note that

$$
G^{\prime}(z)=-f-\Psi(\alpha-z)-\Psi(\alpha+z), \quad G^{\prime \prime}(z)=\Psi_{1}(\alpha-z)-\Psi_{1}(\alpha+z),
$$

where

$$
\Psi(z)=\frac{d}{d z} \log (\Gamma(z)), \quad \Psi_{n}(z)=\frac{d^{n}}{d z^{n}} \Psi(z) .
$$

If we set $f=-2 \Psi(\alpha)$, then $G^{\prime}(0)=G^{\prime \prime}(0)=0$ and we can use the Taylor expansion around zero

$$
G(z)=\sigma^{3} z^{3} / 3+\mathcal{O}\left(z^{4}\right),
$$

where $\sigma^{3}=G^{\prime \prime \prime}(0) / 2$. Hence we find that $G$ has a double critical point at zero, which means that when $\alpha_{\circ} \geqslant 0$, we may use a saddle-point method around this critical point. For the Laplace's method to work, one also needs to control the decay of $\mathfrak{R e}[G(z)]$ along the contours. However, when $\alpha_{\circ}<0$, this is not possible and the asymptotic behavior of (171) will be quite different.

We will first provide probabilistic heuristics explaining why we should expect a phase transition at $\alpha_{\circ}=0$. Then we show that a formal asymptotic analysis of (171)_following ideas similar to [BCF14, BCR13, KQ18] though these works are rigorous-confirms all these heuristics.

8.1. The Baik-Rains phase transition for directed polymers. Consider the partition function of the half-space log-gamma polymer with weights $w_{i, j} \sim$ $\operatorname{Gamma}^{-1}(2 \alpha)$ for $i>j$ and $w_{i, i} \sim \operatorname{Gamma}^{-1}\left(\alpha_{\circ}+\alpha\right)$. In order to understand why the asymptotic behavior of $Z(n, n)$ is different whether $\alpha_{\circ} \geqslant 0$ or $\alpha_{\circ}<0$, let us examine the asymptotics of the free energy

$$
\log (Z(n, n))=\log \left(\sum_{\text {path } \pi} \prod_{(i, j) \in \pi} w_{i, j}\right) .
$$


By KPZ universality, we expect that this quantity has a deterministic first order linear in $n$ and fluctuations on the scale $n^{1 / 3}$. Only a small fraction of admissible paths contribute to the first order of the free energy. Let $H(\pi)=$ $\sum_{(i, j) \in \pi} \log \left(w_{i, j}\right)$ be the energy of a path. The paths contributing to the first order are those with (close to) maximal energy. The expectation of the energy of a path depends on the number of times the path hits the boundary. It is reasonable to expect that if $\alpha_{\circ} \geqslant \alpha$, the weights on the diagonal will be not larger than the bulk weights (in average) and will not influence much the limiting behavior. However, if $\alpha_{\circ}$ is very small, the weights on the boundary will be typically very large so that the path with maximal energy will typically take $\mathcal{O}(n)$ weights on the boundary. This will increase the first order of the free energy and the Gaussian fluctuations of those boundary weights will imply Gaussian fluctuations for the free energy.

By analogy with the zero-temperature case (see [BBCS18b, Section 6.1]), we expect that for homogeneous weights, the first order of the free energy is the same in a half-quadrant or a full quadrant. (This may also be derived, at least formally, from saddle-point asymptotics of (171).) That is, for $\alpha_{\circ}=\alpha$ (and consequently $\alpha_{\circ} \geqslant \alpha$ ), we expect that (using [Sep12, Theorem 2.4])

$$
\frac{\log (Z(n s, n t))}{n} \underset{n \rightarrow \infty}{\longrightarrow}-s \Psi(\theta)-t \Psi(2 \alpha-\theta),
$$

where $\theta$ is such that

$$
\frac{s}{t}=\frac{\Psi_{1}(2 \alpha-\theta)}{\Psi_{1}(\theta)} .
$$

However, for $\alpha_{\circ}$ sufficiently small, the situation may be different. For instance, when $\alpha_{\circ}$ is close to $-\alpha$, the boundary weights become huge and will dominate the asymptotic behavior of the partition function. In order to predict the precise value of $\alpha_{\circ}$ where the transition arises, the following proposition will be useful. A similar argument is presented in the zero-temperature limit (exponential lastpassage percolation in a half-quadrant) in [BBCS18b, Section 6.1].

PROPOSITION 8.1. Let $a, b>0$. Let $Z_{1}(n, n)$ be the partition function of the half-space log-gamma polymer as in Definition 1.1, where the parameters are chosen so that $\alpha_{1}=\cdots=\alpha_{n}=a / 2>0$ and $\alpha_{\circ}=b-a / 2$ is arbitrary. Let $Z_{2}(n, n)$ be the partition function of the half-space log-gamma polymer where the parameters are chosen as $\alpha_{1}=b-a / 2, \alpha_{2}=\cdots=\alpha_{n}=a / 2$ and $\alpha_{\circ}=a / 2$. Then we have

$$
Z_{1}(n, n) \stackrel{(d)}{=} Z_{2}(n, n)
$$

This proposition means that for the half-space log-gamma polymer, $Z(n, n)$ has the same distribution whether the boundary weights are $\operatorname{Gamma}^{-1}(b)$ and 
the bulk weights are $\mathrm{Gamma}^{-1}(a)$; or when the weights on the first row are $\mathrm{Gamma}^{-1}(b)$ while all other weights are $\mathrm{Gamma}^{-1}(a)$. This is a consequence of the more general identity in law in Proposition 2.6.

Proof. Let $\lambda$ be distributed as $\mathbb{P}_{\left(q^{a / 2}, \ldots, q^{a / 2}\right), q^{b-a / 2}}^{q}$ and $\pi$ be distributed as $\mathbb{P}_{\left(q^{b-a / 2}, q^{a / 2}, \ldots, q^{a / 2}\right), 0}^{q}$, where $q^{a / 2}$ appears $n$ times in both measures. From Proposition 2.6, we know that the distributions of $\lambda_{1}$ and $\pi_{1}$ are the same. We will exploit this fact in the $q \rightarrow 1$ limit.

By Proposition 6.27, the law of $(1-q)^{2 n-1} q^{-\lambda_{1}}$ under $\mathbb{P}_{\left(q^{a / 2}, \ldots, q^{a / 2}\right), q^{b-a / 2}}^{q}$ converges to that of $Z_{1}(n, n)$. Let $\kappa$ be distributed as $\mathbb{P}_{\left(q^{b-a / 2}, q^{a / 2}, \ldots, q^{a / 2}\right), q^{a / 2}}^{q}$, where $q^{a / 2}$ appears $n-1$ times in the specialization $\left(q^{b-a / 2}, q^{a / 2}, \ldots, q^{a / 2}\right)$. One can couple $\pi$ and $\kappa$, by sampling first $\kappa$ and then $\pi$ according to the transition operator $\mathcal{U}^{L}(\pi \mid \kappa)$ from Section 4.2. By Lemma 4.15, we know that $\pi_{1}=$ $\kappa_{1}+\mathrm{qGeom}(0)=\kappa_{1}$. Moreover by Proposition 6.27, the law of $(1-q)^{2 n-1} q^{-\kappa_{1}}$ under $\mathbb{P}_{\left(q^{a / 2}, \ldots, q^{a / 2}\right), q^{b-a / 2}}^{q}$ converges to that of $Z_{2}(n, n)$. Since $(1-q)^{2 n-1} q^{-\lambda_{1}} \stackrel{(d)}{=}$ $(1-q)^{2 n-1} q^{-\kappa_{1}}$, it suffices to let $q$ tend to 1 on both sides to conclude the proof.

Consider now the partition function $Z_{2}(n, n)$ of the half-space log-gamma polymer with weights $w_{i, j} \sim \operatorname{Gamma}^{-1}(2 \alpha)$ for $i \geqslant j>1$ and $w_{i, 1} \sim$ $\mathrm{Gamma}^{-1}\left(\alpha_{\circ}+\alpha\right)$. By Proposition 8.1, $Z(n, n) \stackrel{(d)}{=} Z_{2}(n, n)$. In the latter model, the energy of a path can be conveniently decomposed as the energy collected along the first row plus the energy collected after the path has departed the first row, that is,

$$
H(\pi)=\sum_{i=1}^{k} \log \left(w_{i 1}\right)+\sum_{(i, j) \in \pi ; j>1} \log \left(w_{i, j}\right) .
$$

Assuming that $k$ (the number of steps in the first row) is roughly the same for all paths that contribute to the first order of the free energy, we get

$$
\log \left(Z_{2}(n, n)\right) \approx \sum_{i=1}^{k} \log \left(w_{i 1}\right)+\log \left(\sum_{\pi^{\prime}:(k, 2) \rightarrow(n, n)} \prod_{(i, j) \in \pi^{\prime}} w_{i, j}\right) .
$$

By the previous discussion on the case with homogeneous weights (174), the second term can be approximated by

$$
\frac{1}{n} \log \left(\sum_{\pi^{\prime}:(k, 2) \rightarrow(n, n)} \prod_{(i, j) \in \pi^{\prime}} w_{i, j}\right) \approx-(1-k / n) \Psi(\theta)-\Psi(2 \alpha-\theta),
$$


where $\theta$ is such that $1-k / n=\frac{\Psi_{1}(2 \alpha-\theta)}{\Psi_{1}(\theta)}$. Hence, since $\mathbb{E}\left[\log \left(w_{i}, 1\right)\right]=\Psi\left(\alpha_{\circ}+\alpha\right)$,

$$
\frac{\mathbb{E} \log (Z(n, n))}{n} \approx \max _{k}\left\{-k / n \Psi\left(\alpha_{\circ}+\alpha\right)-(1-k / n) \Psi(\theta)-\Psi(2 \alpha-\theta)\right\} .
$$

A quick study of the function

$$
\begin{gathered}
x \mapsto-x \Psi\left(\alpha_{\circ}+\alpha\right)-(1-x) \Psi(\theta)-\Psi(2 \alpha-\theta) \text { under the constraint } \\
1-x=\frac{\Psi_{1}(2 \alpha-\theta)}{\Psi_{1}(\theta)}
\end{gathered}
$$

when $x \in[0,1]$ shows that when $\alpha_{\circ} \geqslant 0$, the maximum arises for $x=0$ while for $\alpha_{\circ}<0$, the maximum arises for $x$ such that $\theta=\alpha+\alpha_{\circ}$.

Thus, we expect that when $\alpha_{\circ} \geqslant 0$,

$$
\log (Z(n, n)) / n \underset{n \rightarrow \infty}{\longrightarrow}-2 \Psi(\alpha)
$$

with $n^{-2 / 3}$ fluctuations. However, when $\alpha_{\circ}<0$, we expect that

$$
\log (Z(n, n)) / n \underset{n \rightarrow \infty}{\longrightarrow}-\Psi\left(\alpha+\alpha_{\circ}\right)-\Psi\left(\alpha-\alpha_{\circ}\right)
$$

with Gaussian fluctuations on the scale $n^{-1 / 2}$. Since $\operatorname{Var}\left[\log \left(\mathrm{Gamma}^{-1}(\theta)\right)\right]=$ $\Psi_{1}(\theta)$, one can even predict that the variance of the Gaussian should be $x \Psi_{1}\left(\alpha+\alpha_{\circ}\right)=\left(1-\left.\frac{\Psi_{1}(2 \alpha-\theta)}{\Psi_{1}(\theta)}\right|_{\theta=\alpha+\alpha_{\circ}}\right) \Psi_{1}\left(\alpha+\alpha_{\circ}\right)=\Psi_{1}\left(\alpha+\alpha_{\circ}\right)-\Psi_{1}\left(\alpha-\alpha_{\circ}\right)$.

8.2. Fredholm Pfaffians and Tracy-Widom distributions. The Pfaffian of a skew-symmetric $2 k \times 2 k$ matrix $A$ is defined by

$$
\operatorname{Pf}(A)=\frac{1}{2^{k} k !} \sum_{\sigma \in \mathcal{S}_{2 k}} \operatorname{sgn}(\sigma) a_{\sigma(1) \sigma(2)} a_{\sigma(3) \sigma(4)} \ldots a_{\sigma(2 k-1) \sigma(2 k)},
$$

where $\operatorname{sgn}(\sigma)$ is the signature of the permutation $\sigma$. Schur's Pfaffian identity states that for any $x_{1}, x_{2}, \ldots, x_{2 n}$,

$$
\operatorname{Pf}\left(\frac{x_{i}-x_{j}}{x_{i}+x_{j}}\right)=\prod_{i<j} \frac{x_{i}-x_{j}}{x_{i}+x_{j}} .
$$

Let $(\mathbb{X}, \mu)$ be a measure space. For a $2 \times 2$ matrix valued skew-symmetric kernel,

$$
\mathbf{K}(x, y)=\left(\begin{array}{ll}
\mathbf{K}_{11}(x, y) & \mathbf{K}_{12}(x, y) \\
\mathbf{K}_{21}(x, y) & \mathbf{K}_{22}(x, y)
\end{array}\right), \quad x, y \in \mathbb{X},
$$


we define the Fredholm Pfaffian (introduced in [Rai00, Section 8]) by

$$
\operatorname{Pf}[\mathbf{J}+\mathbf{K}]_{L^{2}(\mathbb{X}, \mu)}=1+\sum_{k=1}^{\infty} \frac{1}{k !} \int_{\mathbb{X}} \ldots \int_{\mathbb{X}} \operatorname{Pf}\left(\mathbf{K}\left(x_{i}, x_{j}\right)\right)_{i, j=1}^{k} d \mu\left(x_{1}\right) \ldots d \mu\left(x_{k}\right),
$$

provided the series converges. The kernel $\mathbf{J}$ is defined by

$$
\mathbf{J}(x, y)=\delta_{x=y}\left(\begin{array}{cc}
0 & 1 \\
-1 & 0
\end{array}\right) .
$$

In what follows, $\mu$ is the Lebesgue measure and we write $\operatorname{Pf}[\mathbf{J}+K]_{\mathbb{L}^{2}(\mathbb{X})}$.

For $a \in \mathbb{R}$ and $\theta \in(0 \pi / 2)$, let $\mathcal{C}_{a}^{\theta}$ be the contour formed by the union of two semi-infinite rays departing $a$ with angles $\theta$ and $-\theta$, oriented from $a+\infty e^{-\mathbf{i} \theta}$ to $a+\infty e^{\mathbf{i} \theta}$.

Definition 8.2. The GSE Tracy-Widom distribution $\mathcal{L}^{\mathrm{GSE}}$ is a continuous probability distribution on the real line such that for $X \sim \mathcal{L}^{\mathrm{GSE}}, F_{\mathrm{GSE}}(x):=$ $\mathbb{P}(X \leqslant x)=\operatorname{Pf}\left(\mathbf{J}-\mathbf{K}^{\mathrm{GSE}}\right)_{\mathbb{L}^{2}(x, \infty)}$, where $\mathbf{K}^{\mathrm{GSE}}$ is a $2 \times 2$ matrix valued kernel defined by

$$
\begin{aligned}
& \mathbf{K}_{11}^{\mathrm{GSE}}(x, y)=\int_{\mathcal{C}_{1}^{\pi / 3}} \frac{d z}{2 \mathbf{i} \pi} \int_{\mathcal{C}_{1}^{\pi / 3}} \frac{d w}{2 \mathbf{i} \pi} \frac{z-w}{4 z w(z+w)} e^{z^{3} / 3+w^{3} / 3-x z-y w}, \\
& \mathbf{K}_{12}^{\mathrm{GSE}}(x, y)=-\mathbf{K}_{21}^{\mathrm{GSE}}(y, x)=\int_{\mathcal{C}_{1}^{\pi / 3}} \frac{d z}{2 \mathbf{i} \pi} \int_{\mathcal{C}_{1}^{\pi / 3}} \frac{d w}{2 \mathbf{i} \pi} \frac{z-w}{4 z(z+w)} e^{z^{3} / 3+w^{3} / 3-x z-y w}, \\
& \mathbf{K}_{22}^{\mathrm{GSE}}(x, y)=\int_{\mathcal{C}_{1}^{\pi / 3}} \frac{d z}{2 \mathbf{i} \pi} \int_{\mathcal{C}_{1}^{\pi / 3}} \frac{d w}{2 \mathbf{i} \pi} \frac{z-w}{4(z+w)} e^{z^{3} / 3+w^{3} / 3-x z-y w} .
\end{aligned}
$$

Note that the kernel $\mathbf{K}^{\mathrm{GSE}}$ has the form

$$
\mathbf{K}^{\mathrm{GSE}}(x, y)=\left(\begin{array}{cc}
A(x, y) & -\partial_{y} A(x, y) \\
-\partial_{x} A(x, y) & \partial_{x} \partial_{y} A(x, y)
\end{array}\right),
$$

where $A(x, y)$ is the smooth and antisymmetric kernel $\mathbf{K}_{11}^{\mathrm{GSE}}(x, y)$. We define another kernel

$$
\mathbf{K}^{\infty}(x, y)=\left(\begin{array}{cc}
A(x, y) & -2 \partial_{y} A(x, y) \\
-2 \partial_{x} A(x, y) & 4 \partial_{x} \partial_{y} A(x, y)+\boldsymbol{\delta}^{\prime}(x, y)
\end{array}\right),
$$

where $\boldsymbol{\delta}^{\prime}$ is a distribution on $\mathbb{R}^{2}$ such that

$$
\iint f(x, y) \boldsymbol{\delta}^{\prime}(x, y) d x d y=\left.\int\left(\partial_{y} f(x, y)-\partial_{x} f(x, y)\right)\right|_{y=x} d x,
$$


for smooth and compactly supported test functions $f$. It is shown in [BBCS18b, Section 5] that the Fredholm Pfaffian of $\mathbf{K}^{\infty}$ is well defined and we have

$$
\operatorname{Pf}\left[\mathbf{J}-\mathbf{K}^{\mathrm{GSE}}\right]_{\mathbb{L}^{2}(x,+\infty)}=\operatorname{Pf}\left[\mathbf{J}-\mathbf{K}^{\infty}\right]_{\mathbb{L}^{2}(x,+\infty)} .
$$

Moreover, if a sequence of kernels $\mathbf{K}_{n}$ converges to $\mathbf{K}^{\infty}$ in a certain sense (see [BBCS18b, Proposition 5.7]), then

$$
\lim _{n \rightarrow \infty} \operatorname{Pf}\left[\mathbf{J}-\mathbf{K}_{n}\right]_{\mathbb{L}^{2}(x,+\infty)}=\operatorname{Pf}\left[\mathbf{J}-\mathbf{K}^{\mathrm{GSE}}\right]_{\mathbb{L}^{2}(x,+\infty)} .
$$

Definition 8.3. The GOE Tracy-Widom distribution $\mathcal{L}^{\mathrm{GOE}}$ is a continuous probability distribution on the real line such that for $X \sim \mathcal{L}_{\mathrm{GOE}}, F_{\mathrm{GOE}}(x):=$ $\mathbb{P}(X \leqslant x)=\operatorname{Pf}\left(\mathbf{J}-\mathbf{K}^{\mathrm{GOE}}\right)_{\mathbb{L}^{2}(x, \infty)}$, where $\mathbf{K}^{\mathrm{GOE}}$ is the $2 \times 2$ matrix valued kernel defined by

$$
\begin{aligned}
\mathbf{K}_{11}^{\mathrm{GOE}}(x, y)= & \int_{\mathcal{C}_{1}^{\pi / 3}} \frac{d z}{2 \mathbf{i} \pi} \int_{\mathcal{C}_{1}^{\pi / 3}} \frac{d w}{2 \mathbf{i} \pi} \frac{z-w}{z+w} e^{z^{3} / 3+w^{3} / 3-x z-y w}, \\
\mathbf{K}_{12}^{\mathrm{GOE}}(x, y)= & -\mathbf{K}_{21}^{\mathrm{GOE}}(y, x)=\int_{\mathcal{C}_{1}^{\pi / 3}} \frac{d z}{2 \mathbf{i} \pi} \int_{\mathcal{C}_{-1 / 2}^{\pi / 3}} \frac{d w}{2 \mathbf{i} \pi} \frac{w-z}{2 w(z+w)} e^{z^{3} / 3+w^{3} / 3-x z-y w}, \\
\mathbf{K}_{22}^{\mathrm{GOE}}(x, y)= & \int_{\mathcal{C}_{1}^{\pi / 3}} \frac{d z}{2 \mathbf{i} \pi} \int_{\mathcal{C}_{1}^{\pi / 3}} \frac{d w}{2 \mathbf{i} \pi} \frac{z-w}{4 z w(z+w)} e^{z^{3} / 3+w^{3} / 3-x z-y w} \\
& +\int_{\mathcal{C}_{1}^{\pi / 3}} \frac{d z}{2 \mathbf{i} \pi} \frac{e^{z^{3} / 3-z x}}{4 z}-\int_{\mathcal{C}_{1}^{\pi / 3}} \frac{d z}{2 \mathbf{i} \pi} \frac{e^{z^{3} / 3-z y}}{4 z}-\frac{\operatorname{sgn}(x-y)}{4},
\end{aligned}
$$

where we adopt the convention that

$$
\operatorname{sgn}(x-y)=\mathbf{1}_{x>y}-\mathbf{1}_{x<y} .
$$

\subsection{Formal saddle-point asymptotics leading to the Baik-Rains transition.} In this section, we explain how a nonrigorous asymptotic analysis of (171) leads to the following.

FORMAL ASYMPTOTICS. Let $Z(n, n)$ be the half-space log-gamma partition function (Definition 1.1) with $\alpha_{1}=\cdots=\alpha_{n}=\alpha>0$ and $\alpha_{\circ} \in \mathbb{R}$. Let us define

$$
f=-2 \Psi(\alpha), \quad \sigma=\sqrt[3]{\Psi_{2}(\alpha)} .
$$

Modulo several nonrigorous steps in the (attempted) proof presented below, we have the following weak limits: 
- When $\alpha_{\circ}>0$,

$$
\lim _{n \rightarrow \infty} \mathbb{P}\left(\frac{\log (Z(n, n))-f n}{\sigma n^{1 / 3}} \leqslant x\right)=F_{\mathrm{GSE}}(x) .
$$

- When $\alpha_{\circ}=0$, we have

$$
\lim _{n \rightarrow \infty} \mathbb{P}\left(\frac{\log (Z(n, n))-f n}{\sigma n^{1 / 3}} \leqslant x\right)=F_{\mathrm{GOE}}(x) .
$$

- When $\alpha_{\circ}<0$,

$$
\lim _{n \rightarrow \infty} \mathbb{P}\left(\frac{\log (Z(n, n))-f_{\alpha} n}{\sigma_{\alpha} n^{1 / 2}} \leqslant x\right)=\int_{-\infty}^{x} \frac{e^{-t^{2} / 2}}{\sqrt{2 \pi}} d t,
$$

where $f_{\alpha_{\circ}}=-\Psi\left(\alpha-\alpha_{\circ}\right)-\Psi\left(\alpha+\alpha_{\circ}\right)$ and $\sigma_{\alpha}=\sqrt{\Psi_{1}\left(\alpha+\alpha_{\circ}\right)-\Psi_{1}\left(\alpha-\alpha_{\circ}\right)}$.

We use Laplace's method and rescale variables around the critical point of the function $G$ in (171). Note that the same function $G$ as in (171) also appears in the asymptotic analysis of the full-quadrant log-gamma polymer [BCR13, KQ18] and can be controlled along certain contours. Since the asymptotic analysis performed in this section is not rigorous anyway, we will not write the relevant decay estimates of $\mathfrak{R e}[G(z)]$. Thus, let us rescale the variables around zero as $z_{i}=\sigma^{-1} n^{-1 / 3} \tilde{z}_{i}$ and $w_{i}=\sigma^{-1} n^{-1 / 3} \tilde{w}_{i}$ in (171) (we will drop the tildes in the following formulas). There are now three cases to consider. If $\alpha_{\circ}>0$, then

$$
\frac{\Gamma\left(\alpha_{\circ}-n^{-1 / 3} \sigma^{-1} z_{i}\right)}{\Gamma\left(\alpha_{\circ}+n^{-1 / 3} \sigma^{-1} v_{i}\right)} \rightarrow 1 .
$$

If $\alpha_{\circ}=0$, then

$$
\frac{\Gamma\left(\alpha_{\circ}-n^{-1 / 3} \sigma^{-1} z_{i}\right)}{\Gamma\left(\alpha_{\circ}+n^{-1 / 3} \sigma^{-1} v_{i}\right)} \rightarrow \frac{-v_{i}}{z_{i}} .
$$

If $\alpha_{\circ}<0$, then there is a pole at $-\alpha_{\circ}$ for the variable $z$ that prevents us from using the saddle-point method around 0 , the scalings will be different, and we will use the saddle-point method around $\alpha_{\circ}$.

8.3.1. Case $\alpha_{\circ}>0$. Using the fact that

$$
\Gamma(z) \approx \frac{1}{z}, \quad \frac{\pi}{\sin (\pi z)} \approx \frac{1}{z}
$$


for $z$ close to zero, the integrand in (171) becomes, as $n$ goes to infinity,

$$
\begin{aligned}
& \prod_{1 \leqslant i<j \leqslant k} \frac{\left(z_{i}-z_{j}\right)\left(v_{i}-v_{j}\right)\left(z_{i}-v_{j}\right)\left(v_{i}-z_{j}\right)}{\left(v_{i}+z_{j}\right)\left(v_{j}+z_{i}\right)\left(v_{i}+v_{j}\right)\left(z_{i}+z_{j}\right)} \prod_{i=1}^{k} \frac{v_{i}-z_{i}}{v_{i}+z_{i}} \\
& \quad \times \prod_{i=1}^{k}\left[e^{v_{i}^{3} / 3+z_{i}^{3} / 3-x z_{i}-x v_{i}} \frac{1}{v_{i}+z_{i}} \frac{1}{2 v_{i}}\right] .
\end{aligned}
$$

Note that for $\left(u_{1}, \ldots, u_{2 k}\right)=\left(v_{1}, z_{1}, \ldots, v_{k}, z_{k}\right)$, and using Schur's Pfaffian identity (176),

$$
\prod_{1 \leqslant i<j \leqslant k} \frac{\left(z_{i}-z_{j}\right)\left(v_{i}-v_{j}\right)\left(z_{i}-v_{j}\right)\left(v_{i}-z_{j}\right)}{\left(v_{i}+z_{j}\right)\left(v_{j}+z_{i}\right)\left(v_{i}+v_{j}\right)\left(z_{i}+z_{j}\right)} \prod_{i=1}^{k} \frac{v_{i}-z_{i}}{v_{i}+z_{i}}=(-1)^{k} \operatorname{Pf}\left(\frac{u_{i}-u_{j}}{u_{i}+u_{j}}\right) .
$$

We use that for $\mathfrak{R e}\left[z_{i}+v_{i}\right]>0$,

$$
\frac{1}{z_{i}+v_{i}}=\int_{0}^{\infty} e^{-r_{i}\left(z_{i}+v_{i}\right)} d r_{i}
$$

We may bring the integrations inside the Pfaffian to find that

$$
\begin{aligned}
\lim _{n \rightarrow \infty} \mathbb{E}\left[e^{u Z(t, n)}\right] & =\sum_{k=0}^{\infty} \frac{(-1)^{k}}{k !} \int_{x}^{\infty} d r_{1} \cdots \int_{x}^{\infty} d r_{k} \operatorname{Pf}\left(K\left(r_{i}, r_{j}\right)\right)_{i, j=1}^{k} \\
& =\operatorname{Pf}[\mathbf{J}-\mathbf{K}]_{\mathbb{L}^{2}(x, \infty)}
\end{aligned}
$$

where $\mathbf{K}$ is the $2 \times 2$ matrix antisymmetric kernel

$$
\begin{aligned}
& \mathbf{K}_{11}(r, s)=\frac{1}{4} \int_{\mathcal{D}_{1}} \frac{d v}{2 \mathbf{i} \pi} \int_{\mathcal{D}_{1}} \frac{d v^{\prime}}{2 \mathbf{i} \pi} \frac{v-v^{\prime}}{v v^{\prime}\left(v+v^{\prime}\right)} e^{v^{3} / 3+v^{\prime 3} / 3-r v-s v^{\prime}}, \\
& \mathbf{K}_{12}(r, s)=\frac{1}{2} \int_{\mathcal{D}_{1}} \frac{d v}{2 \mathbf{i} \pi} \int_{\mathcal{D}_{-1 / 2}} \frac{d z}{2 \mathbf{i} \pi} \frac{v-z}{v(v+z)} e^{v^{3} / 3+z^{3} / 3-r v-s z}, \\
& \mathbf{K}_{22}(r, s)=\int_{\mathcal{D}_{-1}} \frac{d z}{2 \mathbf{i} \pi} \int_{\mathcal{D}_{-1}} \frac{d z^{\prime}}{2 \mathbf{i} \pi} \frac{z-z^{\prime}}{z+z^{\prime}} e^{z^{3} / 3+z^{\prime 3} / 3-r z-s z^{\prime}} .
\end{aligned}
$$

Note that the formulas above do not make sense because the integrand in (180b) and $(180 c)$ is not absolutely convergent on $\mathcal{D}_{-1}$ and $\mathcal{D}_{-1 / 2}$, respectively. The integration over $\mathcal{D}_{-1 / 2}$ in $(180 b)$ is not a real issue since the contour could have been freely deformed earlier to $\mathcal{D}_{1}$ (the only pole in $z$ is at $z=-v$, which lies on the left of $\left.\mathcal{D}_{-1 / 2}\right)$. However, the integrations over $\mathcal{D}_{-1}$ in $(180 c)$ are a real issue 
because the contours cannot be deformed due to the pole at $z=-z^{\prime}$. However, formally, one can write

$$
\mathbf{K}_{22}(x, y)=\int_{0}^{\infty}\left(\operatorname{Ai}(x-u) \operatorname{Ai}^{\prime}(y-u)-\operatorname{Ai}^{\prime}(x-u) \operatorname{Ai}(y-u)\right) d u,
$$

and using the formal identity

$$
\int_{\mathbb{R}} \operatorname{Ai}(x+u) \operatorname{Ai}(y+u)=\delta(x-y),
$$

one can write $\mathbf{K}_{22}$ as

$$
\mathbf{K}_{22}(x, y)=\int_{\mathcal{D}_{1}} \frac{d z}{2 \mathbf{i} \pi} \int_{\mathcal{D}_{1}} \frac{d z^{\prime}}{2 \mathbf{i} \pi} \frac{z-z^{\prime}}{z+z^{\prime}} e^{z^{3} / 3+z^{\prime 3} / 3-x z-y z^{\prime}}+\boldsymbol{\delta}^{\prime}(x, y),
$$

where $\delta^{\prime}$ is a distribution on $\mathbb{R}^{2}$ defined by (178). Then it follows from (179) in Section 8.2 that

$$
\operatorname{Pf}[\mathbf{J}-\mathbf{K}]_{\mathbb{L}^{2}(x, \infty)}=F_{\mathrm{GSE}}(x) .
$$

Finally, we obtain-modulo several nonrigorous steps above-that when $\alpha_{\circ}>0$ and $\tau>0$,

$$
\lim _{n \rightarrow \infty} \mathbb{P}\left(\frac{\log (Z(n, n, \tau))-f n}{\sigma n^{1 / 3}} \leqslant x\right)=F_{\mathrm{GSE}}(x),
$$

where $f=-2 \Psi(\alpha)$ and $\sigma=\sqrt[3]{\Psi_{2}(\alpha)}$.

8.3.2. Case $\alpha_{\circ}=0$. Following the same steps as in Section 8.3.1, we obtain when $\alpha_{\circ}=0$ that

$$
\begin{aligned}
\lim _{n \rightarrow \infty} \mathbb{E}\left[e^{u Z(t, n)}\right] & =\sum_{k=0}^{\infty} \frac{(-1)^{k}}{k !} \int_{x}^{\infty} d r_{1} \cdots \int_{x}^{\infty} d r_{k} \operatorname{Pf}\left(\mathbf{K}\left(r_{i}, r_{j}\right)\right)_{i, j=1}^{k} \\
& =\operatorname{Pf}[J+\mathbf{K}]_{\mathbb{L}^{2}(x, \infty)},
\end{aligned}
$$

where $K$ is the $2 \times 2$ matrix antisymmetric kernel

$$
\begin{aligned}
& \mathbf{K}_{11}(r, s)=\frac{1}{4} \int_{\mathcal{D}_{1}} \frac{d v}{2 \mathbf{i} \pi} \int_{\mathcal{D}_{1}} \frac{d v^{\prime}}{2 \mathbf{i} \pi} \frac{v-v^{\prime}}{\left(v+v^{\prime}\right)} e^{v^{3} / 3+v^{\prime 3} / 3-r v-s v^{\prime}}, \\
& \mathbf{K}_{12}(r, s)=\frac{1}{2} \int_{\mathcal{D}_{1}} \frac{d v}{2 \mathbf{i} \pi} \int_{\mathcal{D}_{-1 / 2}} \frac{d z}{2 \mathbf{i} \pi} \frac{v-z}{z(v+z)} e^{v^{3} / 3+z^{3} / 3-r v-s z}, \\
& \mathbf{K}_{22}(r, s)=\int_{\mathcal{D}_{-1}} \frac{d z}{2 \mathbf{i} \pi} \int_{\mathcal{D}_{-1}} \frac{d z^{\prime}}{2 \mathbf{i} \pi} \frac{z-z^{\prime}}{z z^{\prime}\left(z+z^{\prime}\right)} e^{z^{3} / 3+z^{\prime 3} / 3-r z-s z^{\prime}} .
\end{aligned}
$$


The formula for $\mathbf{K}_{22}$ in (181c) does not make sense but if one shifts the vertical contours to the right so that they become $\mathcal{D}_{1}$ and compute the residues, then the resulting formula does make sense. Moreover, $\operatorname{Pf}[J+\mathbf{K}]_{\mathbb{L}^{2}(x, \infty)}=\operatorname{Pf}[\mathbf{J}-$ $\tilde{\mathbf{K}}]_{\mathbb{L}^{2}(x, \infty)}$, where $\mathbf{K}_{11}=\tilde{\mathbf{K}}_{11}, \mathbf{K}_{22}=\tilde{\mathbf{K}}_{22}, \mathbf{K}_{12}=-\tilde{\mathbf{K}}_{12}$ and $\mathbf{K}_{21}=-\tilde{\mathbf{K}}_{21}$, and it can be shown (see [BBCS18b, Lemma 2.6]) that

$$
\operatorname{Pf}[\mathbf{J}-\tilde{\mathbf{K}}]_{\mathbb{L}^{2}(x, \infty)}=F_{\mathrm{GOE}}(x) .
$$

Thus when $\alpha_{\circ}=0$ and $\tau>0$, modulo several nonrigorous steps,

$$
\lim _{n \rightarrow \infty} \mathbb{P}\left(\frac{\log (Z(n, n, \tau))-f n}{\sigma n^{1 / 3}} \leqslant x\right)=F_{\mathrm{GOE}}(x),
$$

where $f=-2 \Psi(\alpha)$ and $\sigma=\sqrt[3]{\Psi_{2}(\alpha)}$.

REMARK 8.4. If we scale $\alpha_{\circ}$ close to the critical point as $\alpha_{\circ}=n^{-1 / 3} \sigma^{-1} \varpi$, the limiting distribution $F_{\mathrm{GOE}}$ would be replaced by a crossover distribution $F(x ; \varpi)$, originally introduced in [BR01b, Definition 4] in the context of halfspace last-passage percolation with geometric weights. This distribution is such that $F(x ; 0)=F_{\mathrm{GOE}}(x)$ and $\lim _{\varpi \rightarrow \infty} F(x ; \varpi)=F_{\mathrm{GSE}}(x)$. The cumulative probability distribution $F(x ; \varpi)$ can be written as the Fredholm Pfaffian of a crossover kernel introduced in [FNR06, (1.14)], which is also a special case of the more general crossover kernel $\mathbf{K}^{\text {cross }}$ in $[\mathbf{B B C S 1 8 b}$, Theorem 1.7 and Definition 2.9].

8.3.3. Case $\alpha_{\circ}<0$. In this case, one cannot deform the contours so that they go through a neighborhood of zero as in Section 8.3.1, because there is a pole in (171) at $z_{i}=\alpha_{\circ}<0$. Instead, we will scale $u$ in a different way and apply the saddle-point method in a neighborhood of $\alpha_{\circ}$.

Let $f_{\alpha_{\circ}}=-\Psi\left(\alpha-\alpha_{\circ}\right)-\Psi\left(\alpha+\alpha_{\circ}\right)$. Letting $u=-e^{-n f_{\alpha_{\circ}}-n^{1 / 2} \sigma_{\alpha_{\circ}} x}$ (where $\sigma_{\alpha_{\circ}}$ is a constant to determine later), we may write

$$
\begin{aligned}
\mathbb{E} & {\left[e^{u Z(n, n)}\right]=\sum_{k=0}^{n} \frac{1}{k !} \int_{\mathcal{D}_{R}} \frac{d z_{1}}{2 \mathbf{i} \pi} \cdots \int_{\mathcal{D}_{R}} \frac{d z_{k}}{2 \mathbf{i} \pi} \oint \frac{d v_{1}}{2 \mathbf{i} \pi} \cdots \oint \frac{d v_{k}}{2 \mathbf{i} \pi} } \\
& \times \prod_{1 \leqslant i<j \leqslant k} \frac{\left(z_{i}-z_{j}\right)\left(v_{i}-v_{j}\right) \Gamma\left(v_{i}+v_{j}\right) \Gamma\left(-z_{i}-z_{j}\right)}{\left(-v_{i}-z_{j}\right)\left(-v_{j}-z_{i}\right) \Gamma\left(v_{j}-z_{i}\right) \Gamma\left(v_{i}-z_{j}\right)} \prod_{i=1}^{k} \frac{\Gamma\left(2 v_{i}\right)}{\Gamma\left(v_{i}-z_{i}\right)} \\
& \times \prod_{i=1}^{k}\left[\frac{\pi}{\sin \left(\pi\left(v_{i}+z_{i}\right)\right)} e^{n\left(G_{\alpha_{\circ}}\left(v_{i}\right)+G_{\alpha_{\circ}}\left(z_{i}\right)\right)-n^{1 / 2} x \sigma\left(v_{i}+z_{i}\right)} \frac{\Gamma\left(\alpha_{\circ}-z_{i}\right)}{\Gamma\left(\alpha_{\circ}+v_{i}\right)} \frac{1}{v_{i}+z_{i}}\right],
\end{aligned}
$$

where

$$
G_{\alpha_{\circ}}(z)=\log (\Gamma(\alpha-z))-\log (\Gamma(\alpha+z))-f_{\alpha_{0}} z
$$


satisfies $G_{\alpha_{\circ}}^{\prime}\left(\alpha_{\circ}\right)=0$ and $G_{\alpha_{\circ}}^{\prime \prime}\left(\alpha_{\circ}\right)=\Psi_{1}\left(\alpha-\alpha_{\circ}\right)-\Psi_{1}\left(\alpha+\alpha_{\circ}\right)=:-\sigma_{\alpha}^{2}$. Assuming that the saddle-point method would work without any issue, we rescale variables $z_{i}$ as $z_{i}=\alpha_{\circ}+\sigma_{\alpha} n^{-1 / 2} \tilde{z}_{i}$ and variables $v_{i}$ as $v_{i}=-\alpha_{\circ}+\sigma_{\alpha} n^{-1 / 2} \tilde{v}_{i}$, and obtain

$$
\begin{aligned}
\mathbb{E}\left[e^{u Z(n, n)}\right] & \approx \sum_{k=0}^{n} \frac{1}{k !} \int_{\mathcal{D}_{-1 / 2}} \frac{d z_{1}}{2 \mathbf{i} \pi} \cdots \int_{\mathcal{D}_{-1 / 2}} \frac{d z_{k}}{2 \mathbf{i} \pi} \int_{\mathcal{D}_{1}} \frac{d v_{1}}{2 \mathbf{i} \pi} \cdots \int_{\mathcal{D}_{1}} \frac{d v_{k}}{2 \mathbf{i} \pi} \\
& \times \prod_{1 \leqslant i<j \leqslant k} \frac{\left(z_{i}-z_{j}\right)\left(v_{i}-v_{j}\right)}{\left(-v_{i}-z_{j}\right)\left(-v_{j}-z_{i}\right)} \\
& \times \prod_{i=1}^{k}\left[\frac{1}{v_{i}+z_{i}} e^{-\left(z_{i}^{2} / 2-w_{i}^{2} / 2\right)-x\left(v_{i}+z_{i}\right)} \frac{-v_{i}}{z_{i}} \frac{1}{v_{i}+z_{i}}\right] .
\end{aligned}
$$

Then using the Cauchy determinant formula

$$
\prod_{1 \leqslant i<j \leqslant k} \frac{\left(z_{i}-z_{j}\right)\left(v_{i}-v_{j}\right)}{\left(-v_{i}-z_{j}\right)\left(-v_{j}-z_{i}\right)}=\operatorname{det}\left(\frac{1}{z_{i}+v_{i}}\right)_{i, j=1}^{k}
$$

we may recognize that $(183) \approx \operatorname{det}\left(I-\mathbf{K}^{G}\right)_{\mathbb{L}^{2}(x,+\infty)}$, where

$$
\mathbf{K}^{G}(r, s)=\int_{\mathcal{D}_{-1 / 2}} \frac{d z}{2 \mathbf{i} \pi} \int_{\mathcal{D}_{1}} \frac{d v}{2 \mathbf{i} \pi} \frac{v}{z(z+v)} e^{-z^{2} / 2+v^{2} / 2-r v-s z}=\frac{1}{\sqrt{2 \pi}} e^{-r^{2} / 4-s^{2} / 4}
$$

so that we would obtain

$$
\lim _{n \rightarrow \infty} \mathbb{P}\left(\frac{\log (Z(n, n, \tau))-f_{\alpha} n}{\sigma_{\alpha} n^{1 / 2}} \leqslant x\right)=\int_{-\infty}^{x} \frac{e^{-t^{2} / 2}}{\sqrt{2 \pi}} d t,
$$

where $f_{\alpha_{\circ}}=-\Psi\left(\alpha-\alpha_{\circ}\right)-\Psi\left(\alpha+\alpha_{\circ}\right)$ and $\sigma_{\alpha}=\sqrt{\Psi_{1}\left(\alpha+\alpha_{\circ}\right)-\Psi_{1}\left(\alpha-\alpha_{\circ}\right)}$.

\section{Acknowledgements}

We are grateful to the anonymous referees for their valuable suggestions. A.B. was partially supported by the National Science Foundation (NSF) grants DMS-1056390, DMS-1607901 and DMS-1664619 and by Fellowships of the Radcliffe Institute for Advanced Study and the Simons Foundation. I.C. was partially supported by the NSF grants DMS-1208998, DMS-1664650 and DMS1811143, the Clay Mathematics Institute through a Clay Research Fellowship, the Poincaré Institute through the Poincaré chair and the Packard Foundation through a Packard Fellowship for Science and Engineering. G.B. was partially supported by the Packard Foundation through I.C.'s fellowship. 


\section{References}

[Agg18] A. Aggarwal, 'Dynamical stochastic higher spin vertex models', Selecta Math. (N.S.) 24(3) (2018), 2659-2735.

[AB19] A. Aggarwal and A. Borodin, 'Phase transitions in the ASEP and stochastic sixvertex model', Ann. Probab. 47(2) (2019), 613-689.

[AKQ14] T. Alberts, K. Khanin and J. Quastel, 'The intermediate disorder regime for directed polymers in dimension 1 + 1', Ann. Probab. 42(3) (2014), 1212-1256.

[ACQ11] G. Amir, I. Corwin and J. Quastel, 'Probability distribution of the free energy of the continuum directed random polymer in $1+1$ dimensions', Comm. Pure Appl. Math. 64(4) (2011), 466-537.

[AAR99] G. E. Andrews, R. Askey and R. Roy, Special Functions, (Cambridge University Press, Cambridge, 1999).

[BBCS18a] J. Baik, G. Barraquand, I. Corwin and T. Suidan, 'Facilitated exclusion process', in The Abel Symposium: Computation and Combinatorics in Dynamics, Stochastics and Control (Springer International Publishing, 2018), 1-35.

[BBCS18b] J. Baik, G. Barraquand, I. Corwin and T. Suidan, 'Pfaffian schur processes and last passage percolation in a half-quadrant', Ann. Probab. 46(6) (2018), 3015-3089.

[BR01a] J. Baik and E. M. Rains, 'Algebraic aspects of increasing subsequences', Duke Math. J. 109(1) (2001), 1-65.

[BR01b] J. Baik and E. M. Rains, 'The asymptotics of monotone subsequences of involutions', Duke Math. J. 109(2) (2001), 205-281.

[BR01c] J. Baik and E. M. Rains, 'Symmetrized random permutations', in Random Matrix Models and their Applications, Mathematical Sciences Research Institute Publications, 40 (Cambridge University Press, Cambridge, 2001), 1-19.

[Bar15] G. Barraquand, 'A phase transition for q-TASEP with a few slower particles', Stochastic Process. Appl. 125(7) (2015), 2674-2699.

[BBCW18] G. Barraquand, A. Borodin, I. Corwin and M. Wheeler, 'Stochastic six-vertex model in a half-quadrant and half-line open asymmetric simple exclusion process', Duke Math. J. 167(13) (2018), 2457-2529.

[BC17] G. Barraquand and I. Corwin, 'Random-walk in beta-distributed random environment', Probab. Theory Related Fields 167(3) (2017), 1057-1116.

[BO11] F. Baudoin and N. O'Connell, 'Exponential functionals of Brownian motion and class-one Whittaker functions', Ann. Inst. Henri Poincaré 47(4) 1096-1120.

[BG97] L. Bertini and G. Giacomin, 'Stochastic Burgers and KPZ equations from particle systems', Comm. Math. Phys. 183(3) (1997), 571-607.

[BBNV18] D. Betea, J. Bouttier, P. Nejjar and M. Vuletić, 'The free boundary Schur process and applications I', Ann. Henri Poincaré 19 (2018), 3663-3742. Springer.

[BWZJ15] D. Betea, M. Wheeler and P. Zinn-Justin, 'Refined Cauchy/Littlewood identities and six-vertex model partition functions: II. Proofs and new conjectures', J. Algebraic Combin. 42(2) (2015), 555-603.

[BZ19] E. Bisi and N. Zygouras, 'Point-to-line polymers and orthogonal Whittaker functions', Trans. Amer. Math. Soc. 371(12) (2019), 8339-8379.

[Bor95] A. Borodin, 'Limit Jordan normal form of large triangular matrices over a finite field', Funct. Anal. Appl. 29(4) (1995), 279-281.

[Bor99] A. Borodin, 'The law of large numbers and the central limit theorem for the Jordan normal form of large triangular matrices over a finite field', J. Math. Sci. 96(5) (1999), 3455-3471.

[Bor11] A. Borodin, 'Schur dynamics of the Schur processes', Adv. Math. 4(228) (2011), 2268-2291. 
[Bor14] A. Borodin, 'Integrable probability', In Proceedings of the ICM, Seoul (2014).

[Bor17] A. Borodin, 'On a family of symmetric rational functions', Adv. Math. 306 (2017), 973-1018.

[Bor18] A. Borodin, 'Stochastic higher spin six vertex model and Macdonald measures', J. Math. Phys. 59(2) (2018), 023301.

[BBC16] A. Borodin, A. Bufetov and I. Corwin, 'Directed random polymers via nested contour integrals', Ann. Phys. 368 (2016), 191-247.

[BBW16] A. Borodin, A. Bufetov and M. Wheeler, 'Between the stochastic six vertex model and Hall-Littlewood processes', J. Combin. Theory Ser. A (2016), arXiv:1611.0948 6.

[BC13] A. Borodin and I. Corwin, 'Discrete time q-taseps', Int. Math. Res. Not. IMRN 2015(2) (2013), rnt206.

[BC14] A. Borodin and I. Corwin, 'Macdonald processes', Probab. Theory Related Fields 158(1-2) (2014), 225-400.

[BC15] A. Borodin and I. Corwin, 'Discrete time $q$-TASEPs', Int. Math. Res. Not. IMRN (2) (2015), 499-537.

[BCF14] A. Borodin, I. Corwin and P. Ferrari, 'Free energy fluctuations for directed polymers in random media in 1+ 1 dimension', Comm. Pure Appl. Math. 67(7) (2014), 1129-1214.

[BCFV15] A. Borodin, I. Corwin, P. Ferrari and B. Vető, 'Height fluctuations for the stationary KPZ equation', Math. Phys. Anal. Geom. 18(1) (2015), Art. 20, 95.

[BCG16] A. Borodin, I. Corwin and V. Gorin, 'Stochastic six-vertex model', Duke Math. J. 165(3) (2016), 563-624.

[BCGS16] A. Borodin, I. Corwin, V. Gorin and S. Shakirov, 'Observables of Macdonald processes', Trans. Amer. Math. Soc. 368(3) (2016), 1517-1558.

[BCPS15a] A. Borodin, I. Corwin, L. Petrov and T. Sasamoto, 'Spectral theory for interacting particle systems solvable by coordinate Bethe ansatz', Comm. Math. Phys. 339(3) (2015), 1167-1245.

[BCPS15b] A. Borodin, I. Corwin, L. Petrov and T. Sasamoto, 'Spectral theory for the q-Boson particle system', Compos. Math. 151 (2015), 1-67.

[BCR13] A. Borodin, I. Corwin and D. Remenik, 'Log-gamma polymer free energy fluctuations via a Fredholm determinant identity', Comm. Math. Phys. 324(1) (2013), 215-232.

[BCR15] A. Borodin, I. Corwin and D. Remenik, 'A classical limit of Noumi's q-integral operator', SIGMA Symmetry Integrability Geom. Methods Appl. 11(0) (2015).

[BCS14] A. Borodin, I. Corwin and T. Sasamoto, 'From duality to determinants for q-TASEP and ASEP', Ann. Probab. 42(6) (2014), 2314-2382.

[BCT17] A. Borodin, I. Corwin and F. Toninelli, 'Stochastic heat equation limit of a $(2+1)-d$ growth model', Comm. Math. Phys. 350(3) (2017), 957-984.

[BF14a] A. Borodin and P. L. Ferrari, 'Anisotropic growth of random surfaces in $2+1$ dimensions', Comm. Math. Phys. 325(2) (2014), 603-684.

[BF14b] A. Borodin and P. L. Ferrari, 'Anisotropic growth of random surfaces in $2+1$ dimensions', Comm. Math. Phys. 325(2) (2014), 603-684.

[BG12] A. Borodin and V. Gorin, Lectures on Integrable Probability, Lecture notes, (St. Petersburg School in Probability and Statistical Physics, 2012), arXiv:1212.3351.

[BG15] A. Borodin and V. Gorin, 'General $\beta$-Jacobi corners process and the Gaussian free field', Comm. Pure Appl. Math. 68(10) (2015), 1774-1844.

[BO17] A. Borodin and G. Olshanski, 'The ASEP and determinantal point processes', Comm. Math. Phys. 353(2) (2017), 853-903. 
[BP14] A. Borodin and L. Petrov, 'Integrable probability: From representation theory to macdonald processes', Probab. Surv. 11 (2014), 1-58.

[BP16] A. Borodin and L. Petrov, 'Nearest neighbor Markov dynamics on Macdonald processes', Adv. Math. 300 (2016), 71-155.

[BP18] A. Borodin and L. Petrov, 'Higher spin six vertex model and symmetric rational functions', Selecta Math. (N.S.) 24(2) (2018), 751-874.

[BR05] A. Borodin and E. M. Rains, 'Eynard-Mehta theorem, Schur process, and their Pfaffian analogs', J. Stat. Phys. 121(3-4) (2005), 291-317.

[BM18] A. Bufetov and K. Matveev, 'Hall-Littlewood RSK field', Selecta Math. (N.S.) 24(5) (2018), 4839-4884.

[BP15] A. Bufetov and L. Petrov, 'Law of large numbers for infinite random matrices over a finite field', Selecta Math. 21(4) (2015), 1271-1338.

[CDR10] P. Calabrese, P. Le Doussal and A. Rosso, 'Free-energy distribution of the directed polymer at high temperature', Europhys. Lett. 90(2)20002 (2010).

[Cor12] I. Corwin, 'The Kardar-Parisi-Zhang equation and universality class', Random Matrices Theory Appl. 1(01) (2012).

[Cor14] I. Corwin, 'Macdonald processes, quantum integrable systems and the KardarParisi-Zhang universality class', Preprint, 2014, arXiv:1403.6877.

[CD18] I. Corwin and E. Dimitrov, 'Transversal fluctuations of the ASEP stochastic six vertex model, and Hall-Littlewood Gibbsian line ensembles', Comm. Math. Phys. 363(2) (2018), 435-501.

[CH14] I. Corwin and A. Hammond, 'Brownian Gibbs property for Airy line ensembles', Invent. Math. 195(2) (2014), 441-508.

[CH16] I. Corwin and A. Hammond, 'KPZ line ensemble', Probab. Theory Related Fields 166(1-2) (2016), 67-185.

[COSZ14] I. Corwin, N. O'Connell, T. Seppäläinen and N. Zygouras, 'Tropical combinatorics and Whittaker functions', Duke Math. J. 163(3) (2014), 513-563.

[CP15] I. Corwin and L. Petrov, 'The q-PushASEP: A new integrable model for traffic in 1+ 1 dimension', J. Stat. Phys. 160(4) (2015), 1005-1026.

[CSS15] I. Corwin, T. Seppäläinen and H. Shen, 'The strict-weak lattice polymer', J. Stat. Phys. 160 (2015), 1027-1053.

[CS18] I. Corwin and H. Shen, 'Open ASEP in the weakly asymmetric regime', Comm. Pure Appl. Math. 71(10) (2018), 2065-2128.

[CT16] I. Corwin and F. Toninelli, 'Stationary measure of the driven two-dimensional $q$ Whittaker particle system on the torus', Electron. Commun. Probab. 21 (2016).

[DT16] A. Dembo and L. Tsai, 'Weakly asymmetric non-simple exclusion process and the Kardar-Parisi-Zhang equation', Comm. Math. Phys. 341(1) (2016), 219-261.

[DF90] P. Diaconis and J. A. Fill, 'Strong stationary times via a new form of duality', Ann. Probab. 18(4) (1990), 1483-1522.

[DGP17] J. Diehl, M. Gubinelli and N. Perkowski, 'The Kardar-Parisi-Zhang equation as scaling limit of weakly asymmetric interacting Brownian motions', Comm. Math. Phys. 354(2) (2017), 549-589.

[Dim18] E. Dimitrov, 'KPZ and Airy limits of Hall-Littlewood random plane partitions', Ann. Inst. Henri Poincaré 54(2) (2018), 640-693.

[Dot10] V. Dotsenko, 'Replica Bethe ansatz derivation of the Tracy-Widom distribution of the free energy fluctuations in one-dimensional directed polymers', J. Stat. Mech. 2010(07) (2010), P07010. 
[FHH+09] B. Feigin, K. Hashizume, A. Hoshino, J. Shiraishi and S. Yanagida, 'A commutative algebra on degenerate CP1 and Macdonald polynomials', J. Math. Phys. 50(9) (2009), 095215.

[FV15] P. L. Ferrari and B. Vető, 'Tracy-Widom asymptotics for q-TASEP', Ann. Inst. Henri Poincaré Probab. Stat. 51(4) (2015), 1465-1485.

[FNR06] P. J. Forrester, T. Nagao and E. M. Rains, 'Correlation functions for random involutions', Int. Math. Res. Not. IMRN 2006 (2006), 89796.

[Ful02] J. Fulman, 'Random matrix theory over finite fields', Bull. Amer. Math. Soc. (N.S.) 39(1) (2002), 51-85.

[GRAS16] N. Georgiou, F. Rassoul-Agha and T. Seppäläinen, 'Variational formulas and cocycle solutions for directed polymer and percolation models', Comm. Math. Phys. 346(2) (2016), 741-779.

[GRASY15] N. Georgiou, F. Rassoul-Agha, T. Seppäläinen and A. Yilmaz, 'Ratios of partition functions for the log-gamma polymer', Ann. Probab. 43(5) (2015), 2282-2331.

[GS13] N. Georgiou and T. Seppäläinen, 'Large deviation rate functions for the partition function in a log-gamma distributed random potential', Ann. Probab. 41(6) (2013), 4248-4286.

[GLO08] A. Gerasimov, D. Lebedev and S. Oblezin, 'Baxter operator and Archimedean Hecke algebra', Comm. Math. Phys. 284(3) (2008), 867-896.

[GLO09] A. Gerasimov, D. Lebedev and S. Oblezin, 'On q-deformed $\mathfrak{g l}_{\ell+1}$-whittaker function', Comm. Math. Phys. 294(1) (2009), 97.

[Gho17a] P. Ghosal, 'Correlation functions of the Pfaffian Schur process using Macdonald difference operators', Preprint, 2017, arXiv:1705.05859.

[Gho17b] P. Ghosal, 'Hall-Littlewood-PushTASEP and its KPZ limit', Preprint, 2017, arXiv: 1 701.07308.

[Giv97] A. Givental, 'Stationary phase integrals, quantum Toda lattices, flag manifolds and the mirror conjecture', Amer. Math. Soc. Transl. Ser. 2180 (1997), 103-115.

[GO09] A. Gnedin and G. Olshanski, 'A $q$-analogue of de Finetti's theorem', Electron. J. Combin. 16(1) (2009), Research Paper 78, 16.

[GKV14] V. Gorin, S. Kerov and A. Vershik, 'Finite traces and representations of the group of infinite matrices over a finite field', Adv. Math. 254 (2014), 331-395.

[GS15] V. Gorin and M. Shkolnikov, 'Multilevel Dyson Brownian motions via Jack polynomials', Probab. Theory Related Fields 163(3-4) (2015), 413-463.

[GZ18] V. Gorin and L. Zhang, 'Interlacing adjacent levels of $\beta$-Jacobi corners processes', Probab. Theory Related Fields 172(3-4) (2018), 915-981.

[Gra17] P. Grange, 'Log-gamma directed polymer with one free end via coordinate Bethe ansatz', J. Stat. Mech. Theory Exp. 2017(7) (2017), 073102.

[GLD12] T. Gueudré and P. Le Doussal, 'Directed polymer near a hard wall and KPZ equation in the half-space', Europhys. Lett. 100(2) (2012), 26006.

[GS92] L. Gwa and H. Spohn, 'Six-vertex model, roughened surfaces, and an asymmetric spin Hamiltonian', Phys. Rev. Lett. 68(6) (1992), 725.

[HQ18] M. Hairer and J. Quastel, 'A class of growth models rescaling to KPZ', Forum Math., Pi 6 (2018).

[Has82] M. Hashizume, 'Whittaker functions on semisimple Lie groups', Hiroshima Math. J. 12(2) (1982), 259-293.

[IS07] T. Ishii and E. Stade, 'New formulas for Whittaker functions on GL( $n, \mathbb{R})$ ', J. Funct. Anal. 244(1) (2007), 289-314.

[Jor82] B. Jorgensen, Statistical Properties of the Generalized Inverse Gaussian Distribution, Lecure Notes in Statistics, 9, (Springer, 1982). 
[KPZ86] M. Kardar, G. Parisi and Y. Zhang, 'Dynamic scaling of growing interfaces', Phys. Rev. Lett. 56 (1986), 889-892.

[Knu70] D. Knuth, 'Permutations, matrices, and generalized young tableaux', Pacific J. Math. 34(3) (1970), 709-727.

[KQ18] A. Krishnan and J. Quastel, 'Tracy-Widom fluctuations for perturbations of the log-gamma polymer in intermediate disorder', Ann. Appl. Probab. 28(6) (2018), 3736-3764.

[Mac95] I. G. Macdonald, Symmetric Functions and Hall Polynomials, vol. 354, (Clarendon Press, Oxford, 1995).

[Mat19] K. Matveev, 'Macdonald-positive specializations of the algebra of symmetric functions: proof of the Kerov conjecture', Ann. of Math. (2) 189(1) (2019), 277-316.

[MP17] K. Matveev and L. Petrov, 'q-randomized Robinson-Schensted-Knuth correspondences and random polymers', Ann. Inst. H. Poincaré D 4(1) (2017), $1-123$.

[NZ16] V. Nguyen and N. Zygouras, 'Variants of geometric RSK, geometric PNG, and the multipoint distribution of the log-gamma polymer', Int. Math. Res. Not. IMRN 2017(15) (2017), 4732-4795.

[NS] M. Noumi and A. Sano, An infinite family of higher-order difference operators that commute with Ruijsenaars operators of type A, unpublished.

[NS12] M. Noumi and J. Shiraishi.

[Nte16] I. Nteka, (2016), 'Positive temperature dynamics on Gelfand-Tsetlin patterns restricted by wall', $\mathrm{PhD}$ Thesis, University of Warwick.

[O'C12] N. O'Connell, 'Directed polymers and the quantum Toda lattice', Ann. Probab. 40(2) (2012), 437-458.

[O'C14] N. O'Connell, Whittaker Functions and Related Stochastic Processes, (MSRI Publications, 2014), arXiv:1201.4849 65.

[OO15] N. O'Connell and J. Ortmann, 'Tracy-Widom asymptotics for a random polymer model with gamma-distributed weights', Electron. J. Probab. 20(25) (2015), 1-18.

[OP13] N. O'Connell and Y. Pei, 'A $q$-weighted version of the Robinson-Schensted algorithm', Electron. J. Probab. 18 (2013).

[OSZ14] N. O'Connell, T. Seppäläinen and N. Zygouras, 'Geometric RSK correspondence, Whittaker functions and symmetrized random polymers', Invent. Math. 197(2) (2014), 361-416.

[OY01] N. O'Connell and M. Yor, 'Brownian analogues of Burke's theorem', Stochastic Process. Appl. 96(2) (2001), 285-304.

[Oko01] A. Okounkov, 'Infinite wedge and random partitions', Selecta Math. (N.S.) 7(1) (2001), 57-81.

[OR03] A. Okounkov and N. Reshetikhin, 'Correlation function of Schur process with application to local geometry of a random 3-dimensional Young diagram', J. Amer. Math. Soc. 16(3) (2003), 581-603.

[OP17] D. Orr and L. Petrov, 'Stochastic higher spin six vertex model and q-TASEPs', Adv. Math. 317 (2017), 473-525.

[OQR17] J. Ortmann, J. Quastel and D. Remenik, 'A Pfaffian representation for flat ASEP', Comm. Pure Appl. Math. 70(1) (2017), 3-89.

[Par19] S. Parekh, 'The KPZ limit of ASEP with boundary', Comm. Math. Phys. 365(2) (2019), 569-649.

[Pov13] A. M. Povolotsky, 'On the integrability of zero-range chipping models with factorized steady states', J. Phys. A 46(46) (2013), 465205. 
[Qua12] J. Quastel, Introduction to KPZ, available online at www.math.toronto.edu/quastel/ survey.pdf, 2012.

[Rai00] E. M. Rains, 'Correlation functions for symmetrized increasing subsequences', Preprint, 2000, arXiv:math/0006097.

[Rai18] E. M. Rains, 'Multivariate quadratic transformations and the interpolation kernel', SIGMA 14(0) (2018), 19-69.

[RY13] D. Revuz and M. Yor, Continuous Martingales and Brownian Motion, Vol. 293, (Springer Science \& Business Media, 2013).

[SI04] T. Sasamoto and T. Imamura, 'Fluctuations of the one-dimensional polynuclear growth model in half-space', J. Stat. Phys. 115(3-4) (2004), 749-803.

[SS10] T. Sasamoto and H. Spohn, 'Exact height distributions for the KPZ equation with narrow wedge initial condition', Nuclear Phys. B 834(3) (2010), 523-542.

[STS93] M. A. Semenov-Tian-Shansky, 'Quantization of open Toda lattices', in Dynamical Systems VII: Integrable Systems Nonholonomic Dynamical Systems, Vol. 16 (Springer, 1993), 226.

[Sep12] T. Seppäläinen, 'Scaling for a one-dimensional directed polymer with boundary conditions', Ann. Probab. 40(1) (2012), 19-73.

[Sta01] E. Stade, 'Mellin transforms of $\operatorname{GL}(n, \mathbb{R})$ Whittaker functions', Amer. J. Math. 123(1) (2001), 121-161.

[TLD14] T. Thiery and P. Le Doussal, 'Log-gamma directed polymer with fixed endpoints via the Bethe ansatz replica', J. Stat. Mech. 2014(10) (2014), P10018.

[TW09] C. A. Tracy and H. Widom, 'Asymptotics in ASEP with step initial condition', Comm. Math. Phys. 290(1) (2009), 129-154.

[TW13a] C. A. Tracy and H. Widom, 'The asymmetric simple exclusion process with an open boundary', J. Math. Phys. 54(10) (2013), 103301.

[TW13b] C. A. Tracy and H. Widom, 'The Bose gas and asymmetric simple exclusion process on the half-line', J. Stat. Phys. 150(1) (2013), 1-12.

[Ven15] V. Venkateswaran, 'Symmetric and nonsymmetric Hall-Littlewood polynomials of type BC', J. Algebraic Comb. 42 (2015), 331-364.

[WZJ16] M. Wheeler and P. Zinn-Justin, 'Refined Cauchy/Littlewood identities and six-vertex model partition functions: III. Deformed bosons', Adv. Math. 299 (2016), 543-600.

[Wu18] X. Wu, 'Intermediate disorder regime for half-space directed polymers', Preprint, 2018, arXiv:1804.09815. 